

\section{Propositions}

1. Manufacturers will never be able to market a real tobacco flavor for electronic cigarettes.

(this thesis)

2. Nobody knows why people are disgusted by the idea of vaping savory flavors such as salmon and cheese.

(this thesis)

3. Scientists need to be great communicators.

4. Scientists inform, policy makers decide.

5. People's truth is often led by personal values instead of facts.

6. There would be no gender inequality without prejudices and assumptions.

Propositions belonging to the thesis entitled:

"The role of flavors in attractiveness of electronic cigarettes".

Erna J.Z. Krüsemann

Wageningen, 16 March 2021 


\title{
The role of flavors in attractiveness of electronic cigarettes
}

\author{
Erna J.Z. Krüsemann
}




\section{Thesis Committee}

\section{Promotor}

Prof. Dr Kees de Graaf

Professor of Sensory Science and Eating Behaviour

Wageningen University \& Research

\section{Co-promotors}

Dr Reinskje Talhout

Senior Scientific Advisor, Centre for Health Protection

National Institute for Public Health and the Environment (RIVM), Bilthoven

Dr Sanne Boesveldt

Associate Professor, Division of Human Nutrition and Health

Wageningen University \& Research

\section{Other members}

Dr Esther A. Croes, Trimbos Institute, Utrecht

Prof. Dr Vincenzo Fogliano, Wageningen University \& Research

Prof. Dr Suchitra Krishnan-Sarin, Yale University School of Medicine, USA

Dr Martijn J. Martena, Ministry of Health, Welfare and Sport, The Hague

This research was conducted under the auspices of the Graduate School VLAG (Advanced Studies in Food Technology, Agrobiotechnology, Nutrition and Health Sciences). 


\title{
The role of flavors in attractiveness of electronic cigarettes
}

\author{
Erna J.Z. Krüsemann
}

\section{Thesis}

Submitted in fulfilment of the requirements for the degree of doctor at Wageningen University

by the authority of the Rector Magnificus,

Prof. Dr A.P.J. Mol,

in the presence of the

Thesis Committee appointed by the Academic Board

to be defended in public

on Tuesday 16 March 2021

at 4 p.m. in the Aula. 
Erna J.Z. Krüsemann

The role of flavors in attractiveness of electronic cigarettes

274 pages

$\mathrm{PhD}$ thesis, Wageningen University, Wageningen, the Netherlands (2021)

With references, with summary in English and Dutch

ISBN: 978-94-6395-653-6

DOI: https://doi.org/10.18174/537112 
"Tell me and I forget. Teach me and I remember. Involve me and I learn."

- Benjamin Franklin 



\section{Contents}

$\begin{array}{lll}\text { Chapter } 1 \text { General introduction } 9 & \text { p. }\end{array}$

$\begin{array}{lll}\text { Chapter } 2 & \text { An e-liquid flavor wheel: A shared vocabulary based on } 27\end{array}$ systematically reviewing e-liquid flavor classifications in literature

Chapter 3 Nearly 20000 e-liquids and 250 unique flavor descriptions:

An overview of the Dutch market based on information from manufacturers

Chapter 4 Comprehensive overview of common e-liquid ingredients and p. 79 how they can be used to predict an e-liquid's flavor category

Chapter 5 GC-MS analysis of e-cigarette refill solutions: A comparison p. 111 of flavoring composition between flavor categories

Chapter 6 E-liquid flavor preferences and individual factors related to p. 147 vaping: A survey among Dutch never-users, smokers, dual users, and exclusive vapers

Chapter 7 Sensory evaluation of e-liquid flavors by smelling and vaping p. 177 yields similar results

Chapter 8 Both non-smoking youth and smoking adults like sweet and p. 201 minty e-liquid flavors more than tobacco flavor

Chapter 9 General discussion

Summary

p. 249

Samenvatting

p. 255

Dankwoord / acknowledgements

p. 261

About the author

p. 269

List of publications

p. 270

Overview of completed training activities

p. 272 



\section{CHAPTER 1}

General introduction

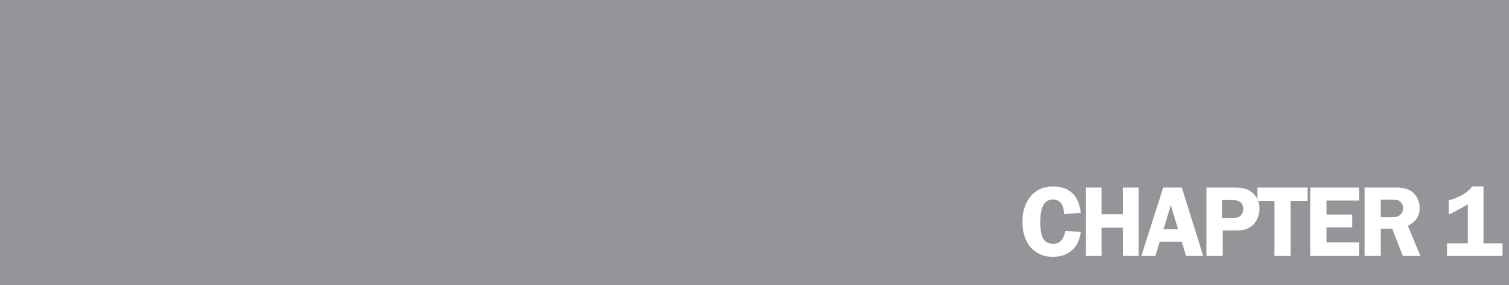


While cigarettes are still by far the number one tobacco product used ${ }^{1}$, the tobacco product market has been expanded in the past decades with several new tobacco and related products. These products are claimed to be less harmful alternatives to cigarettes as they do not require combustion but use a heating system instead ${ }^{2}$. An example of such a product is the electronic cigarette (e-cigarette). Most people say the e-cigarette was invented by the Chinese pharmacist Hon Lik in 2003. According to his patent application, this device is an "electronic atomization cigarette that functions as substitute for quitting smoking and cigarette substitutes" 3 . His e-cigarette is often seen as a disruptive technology that competes with cigarettes and makes combustible tobacco obsolete ${ }^{4-6}$. However, already in 1990, tobacco company Phillip Morris developed a nicotine aerosol technology similar to the modern e-cigarette ${ }^{7}$. According to tobacco industry documents, Phillip Morris aimed for a product that would complement rather than replace cigarettes ${ }^{8}$. The idea was to develop a product that resembles a regular cigarette, but does not emit smoke, and would therefore be a more socially acceptable alternative and of interest to health-concerned smokers. However, uncertainty of how this product might influence future regulation of tobacco products made them use the aerosol technology for pharmaceutical applications instead. Eventually, after introduction of Lik's product into the European and North American markets, e-cigarette sales rapidly increased around 2007-2008 ${ }^{6}$.

The e-cigarette design has evolved strongly ever since and does not necessarily resemble a regular cigarette anymore (Figure 1.1 $)^{9}$. Therefore, e-cigarettes are also called electronic nicotine delivery systems (ENDS). Other terms used for e-cigarettes are e-smokers, vaping devices, vape pens, flavor vapes, e-hookahs, and sisha-pens. E-cigarettes contain a battery and a heating element, which is activated by the user pushing a button or inhaling through the mouthpiece. As a result, a so-called e-liquid, stored in a disposable cartridge or refillable reservoir, is atomized (i.e., vaporized). E-liquids are available with and without nicotine, and further consist of a basis of propylene glycol and/or vegetable glycerin, and, in most cases, flavorings. The user inhales the vapor (i.e., aerosol) into the lungs where nicotine is delivered. Therefore, the use of e-cigarettes is also called "vaping".

Currently, the effect of e-cigarettes on public health is an important topic of debate. On one hand, e-cigarettes are shown to be less harmful to health than combustible tobacco ${ }^{10,11}$ and could be a successful tool in smoking cessation ${ }^{12,13}$. Today, there are still 1.1 billion tobacco smokers in the world ${ }^{1}$, who suffer from an increased risk of death from severe diseases such as cancers (mostly lung cancer), cardiovascular diseases (such as stroke and ischemic heart disease), and respiratory diseases ${ }^{14}$. Tobacco smoking is also associated with an increased risk of several communicable diseases, such as fetal stillbirth, congenital malformations, the sudden infant death syndrome, and respiratory diseases in childhood and adolescence. The fact that tobacco causes more than 8 million deaths each year makes tobacco one of the biggest public health threats in the world's history ${ }^{15}$. Smokers who aim to quit smoking in order to improve their health could switch towards the use of e-cigarettes. In this way, e-cigarettes may serve as a harm reduction tool and promotion of these products could be beneficial to public health ${ }^{9}$. On the other hand, as e-cigarettes are addictive and definitely not harmless ${ }^{10,11}$, they will cause more public harm if people who would otherwise not smoke (non-smokers) start using e-cigarettes. E-cigarette use among youth non-smokers is also associated with a greater risk of subsequent initiation of cigarette smoking ${ }^{16}$. In addition, if people who simultaneously smoke 
and use e-cigarettes (dual users) continue doing both, the toxicant levels they are exposed to will be greater than for those solely smoking combustible cigarettes ${ }^{17}$. This shows that promotion of e-cigarettes may also negatively affect public health ${ }^{9}$.

One of the most important factors that makes e-cigarettes attractive for both smokers and non-smokers is the landscape of available e-liquid flavors, which is the topic of this thesis. This general introduction firstly describes prevalence of e-cigarette use, followed by an overview of product characteristics that contribute to attractiveness, addictiveness, and toxicity of e-cigarettes. The next section focusses on regulation of e-cigarettes and e-liquids, including regulation of flavors. Finally, the aim and subsequent research questions of this thesis project are introduced.

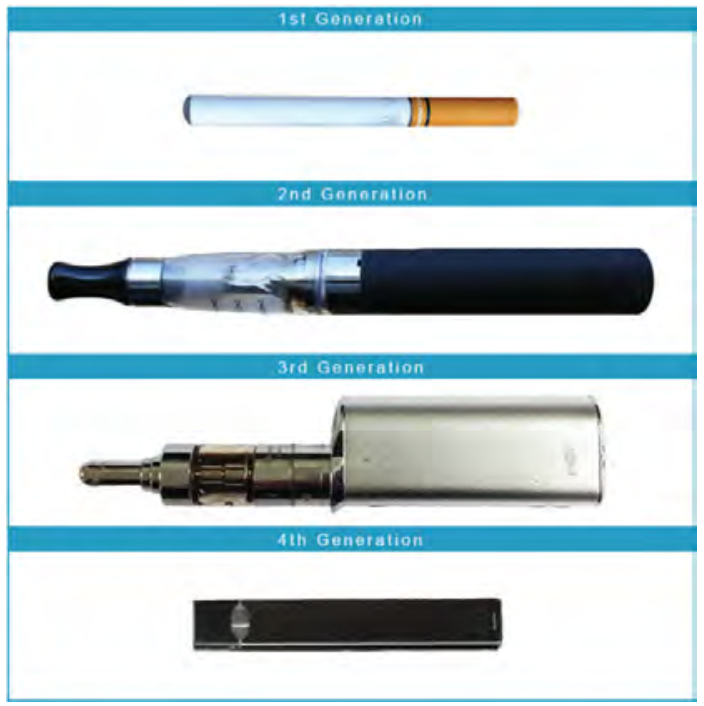

Figure 1.1: Different generations of e-cigarette designs. Image: (C) The Journal of the Royal College of Physicians of Edinburgh, 2018. Originally published in Mathur A, Dempsey OJ. Electronic cigarettes: a brief update. J R Coll Physicians Edinb 48(4):346-351, DOI: 10.4997/JRCPE.2018.415. 


\section{Prevalence of e-cigarette use}

E-cigarettes have been available in the Netherlands since 2007. Ten years later, at the start of this research project, $3.1 \%$ of the Dutch adults occasionally used an e-cigarette ${ }^{18}$. This number was stable between 2016 and $2018^{18}$. Furthermore, e-cigarettes were and are still by far the most used by people who simultaneously smoke tobacco cigarettes as compared to former and nonsmokers ${ }^{18,19}$.

Although 18 is the minimum age for legally purchasing e-cigarettes in the Netherlands ${ }^{20}$, $6 \%$ of the primary school students reported to have ever used an e-cigarette in $2017^{21}$. Among secondary school students, this number increased between the ages of 12 and 16 from $13 \%$ to $36 \%{ }^{21}$. Whereas the average percentage of secondary school students who had ever used an e-cigarette decreased from 2015 to 2017, this percentage was higher than the percentage of students who had ever smoked a tobacco cigarette in both years (34\% vs. $23 \%$ in $2015 ; 28 \%$ vs. $17 \%$ in 2017) ${ }^{21}$.

In the United States (US), there was a substantial increase in the number of high school students who reported current e-cigarette use during 2017-2018 (from 12\% to 21\%; defined as using one or more e-cigarettes in the past 30 days) ${ }^{22}$. This may be attributed to the popularity of a new product called JUUL, which is an e-cigarette in the discreet shape of a USB flash drive (similar to the fourth generation of e-cigarettes displayed in Figure 1.1) and contains an extremely high nicotine concentration and various flavors that attract youth ${ }^{23}$. Prevalence of e-cigarette use by youth in the US is still increasing: $28 \%$ of the high school students (aged 1418 ) and $11 \%$ of middle school students (aged 11-13) reported current e-cigarette use in 2019, and more than half of them reported JUUL as their usual brand ${ }^{24}$. Following the rapid increase in popularity, e-cigarette use among youth was declared an epidemic in 2018 by the US Food and Drug Administration Commissioner and the US Surgeon General ${ }^{25,26}$. The alarming situation in the US and prevalence of e-cigarette use in the Netherlands are reasons for concern about e-cigarette use among youth.

\section{E-cigarette product characteristics}

The reason why experimentation and initiation of e-cigarette use may be of concern, is the effect of e-cigarette use on health. E-cigarette use is a result of the interaction between a product and a person (or: individual) within a certain environment (or: context) ${ }^{27,28}$. This means that e-cigarette use and thus related health effects are influenced by environmental, product, and personal factors (Figure 1.2). Examples of environmental factors are peer use, marketing and advertisement, product accessibility, and legal restrictions on use. Product factors include the design and price of e-cigarettes, and the flavoring, nicotine, and toxicant content of e-liquids. Examples of personal factors are peer susceptibility, sensory perception, nicotine dependence, gender, and age. The interaction between product and personal factors determine people's attitude towards e-cigarettes, including product appeal and perceived harm. The current section elaborates on how product factors, mainly flavorings, contribute to attractiveness, addictiveness, and toxicity of e-cigarettes, thereby playing an important role in e-cigarette use and related health risks. 


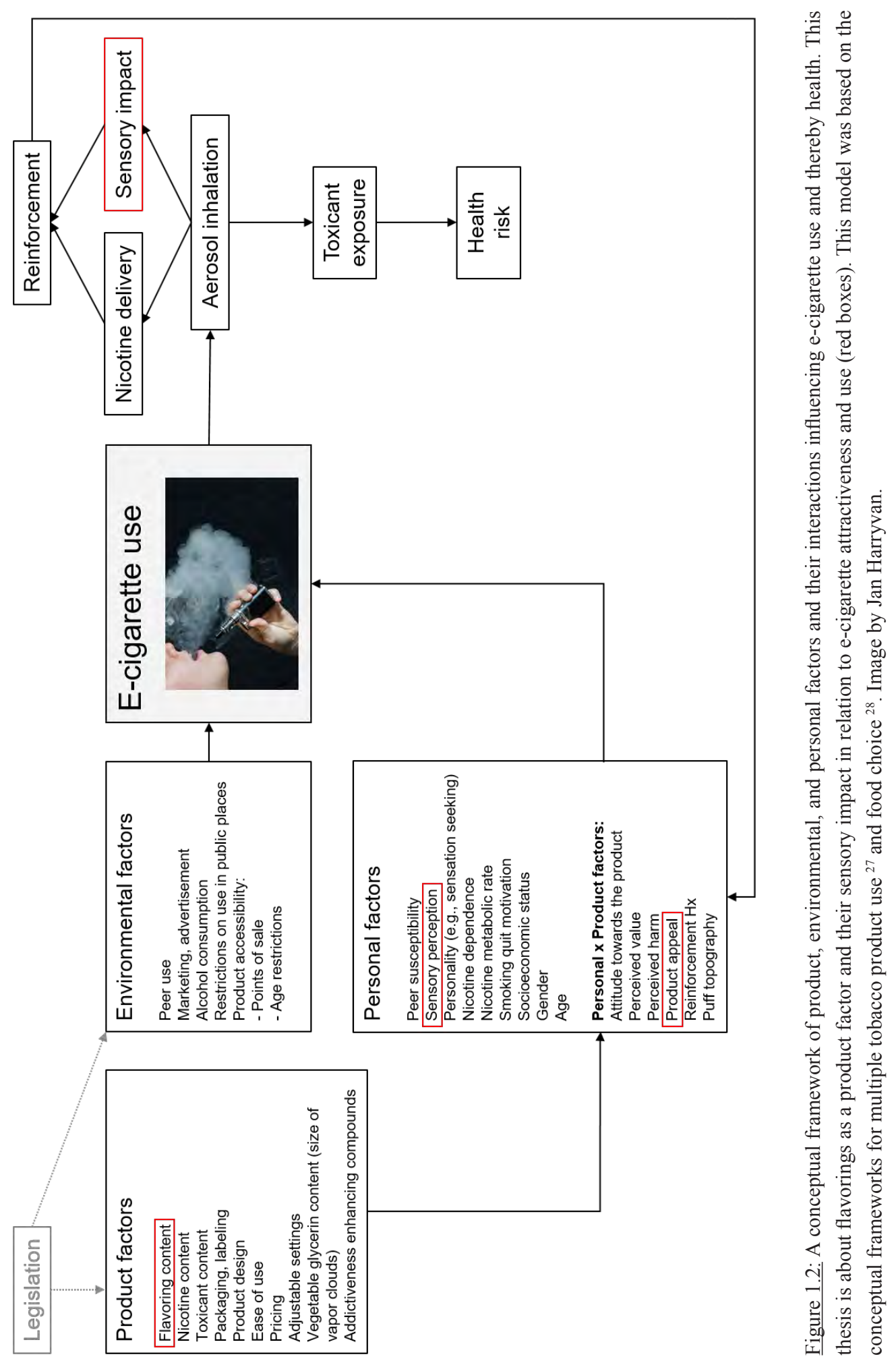




\section{E-cigarette attractiveness}

Attractiveness (or: appeal) of e-cigarettes is stimulated by factors such as packaging and labeling, design of the product, ease of use, the ability to customize settings of the device, and pricing of e-cigarettes and e-liquids ${ }^{29,30}$. The most important factor that contributes to e-cigarette attractiveness is the flavoring content of e-liquids. That is, the availability of a wide variety of palatable e-liquid flavors increases sensory appeal of the product, plays a great role in e-cigarette experimentation ${ }^{31}$, and is often reported as an important reason for initiation and continuation of e-cigarette use ${ }^{32,33}$. Flavors are not only important to current e-cigarette users ${ }^{29-31,34,35}$ and smokers who may want to switch towards e-cigarette use ${ }^{31,36}$, but also to (young) non-smokers ${ }^{36-39}$. For example, a focus group study conducted in the Netherlands showed that all user groups considered the variety of e-liquid flavors a risk for initiation of vaping among youth ${ }^{40}$. Moreover, in the US, $72 \%$ of the high school students who exclusively used e-cigarettes used a flavored one in 2019, with the most commonly reported flavors being fruit, menthol, candy, and dessert ${ }^{24}$. Research also showed that flavors influence perceived harm of e-cigarettes among youth: e-liquids with fruit and candy flavors are perceived as less harmful compared to tobacco flavored e-liquids ${ }^{41-43}$.

\section{E-cigarette toxicity}

While e-cigarettes have attractive characteristics that stimulate their use and may alter perceived harm, they also have addictive and toxic properties. Although the long term health effects of e-cigarettes are largely unknown, e-cigarette vapor contains carcinogenic and other toxic compounds such as carbonyls, volatile organic compounds, tobacco-specific nitrosamines and heavy metals ${ }^{10,11}$. In vitro studies showed that e-cigarette aerosols induce toxicity, inflammatory response, a decreased metabolic activity, and oxidative stress in human bronchial and lung epithelial cells ${ }^{44-46}$. Moreover, endothelial disfunction and increased levels of oxidative stress and inflammation in response to e-cigarette aerosol exposure have been observed in vascular and cerebral cells ${ }^{47}$. Furthermore, some e-liquid constituents other than nicotine may be developmentally toxic, suggesting a health risk of e-cigarettes use for pregnant women ${ }^{48}$. E-cigarette users reported in an online survey to experience several undesirable effects from vaping such as a dry mouth, bad breath, worsening of respiratory symptoms, nicotine-related side effects (e.g., headache), and throat and nasal irritations ${ }^{29}$. Nevertheless, these effects may be considered less severe compared to the type of diseases related to cigarette smoking. In addition, research showed that the number and levels of toxic and potentially toxic compounds found in e-cigarette vapor are much lower than the levels found in cigarette smoke ${ }^{10,11}$.

Flavoring chemicals identified in e-cigarette liquids and vapor, while generally recognized as safe for food products, significantly contribute to inhalation toxicity ${ }^{44,49-52}$. Moreover, chemical reactions may take place when e-liquid constituents are heated, which may result in the formation of secondary and tertiary reaction products. For example, research showed that flavorings contribute to the formation of toxic aldehydes (e.g., formaldehyde, acetaldehyde, acrolein) during vaping ${ }^{53}$. Taken together, it can be concluded that e-cigarettes are generally less harmful than combustible cigarettes but definitely not safe ${ }^{17,54}$. 


\section{E-cigarette addictiveness}

Similar to tobacco products, e-cigarette emissions contain the highly addictive compound nicotine. E-cigarette users can adjust nicotine delivery in devices where settings such as wattage and temperature can be customized (Figure 1.1, third generation of e-cigarettes) ${ }^{55}$. Nicotine drives continued use of the product by stimulating the reward system in the brain ${ }^{56}$. That is, nicotine binds to nicotinic acetylcholine receptors (nAChR), which results in the release of dopamine. Dopamine causes a feeling of pleasure and reinforces the type of behavior that has led to this rewarding goal, in this case, e-cigarette use. This may lead to repeated use of the product and development of addiction (i.e., dependence) over time. Nicotine dependence is particularly concerning among young people: development of the human brain continues until the mid-20s, and nicotine exposure during this vulnerable period is associated with several adverse health effects such as impaired development of the cerebral cortex and hippocampus ${ }^{57}$. Another concern is that e-cigarette experimentation and use among youth is associated with a greater risk of subsequent initiation of cigarette smoking ${ }^{16,58}$, even when they had no previous intention to start smoking ${ }^{59}$. This suggests that e-cigarettes may serve as a gateway to future use of combustible tobacco products, which are equally addictive and even more harmful to health.

Research showed that flavorings enhance the rewarding and reinforcing effects of nicotine in e-cigarettes in young adult smokers ${ }^{60,61}$. For example, menthol and the green apple flavoring, farnesene, facilitate nicotine dependence through upregulation of $\mathrm{nACh}$ receptors in the brain ${ }^{62,63}$. Furthermore, flavorings reduce the nicotine metabolism (e.g., menthol, cinnamaldehyde and benzaldehyde) ${ }^{64,65}$ and are known to facilitate inhalation and nicotine uptake due to their cooling and bronchodilating effects (e.g., menthol, theobromine and eucalyptol) ${ }^{62}$. In addition, flavorings such as vanillin, ethyl vanillin, and coumarin inhibit monoamine oxidase enzymes, which results in a delayed degradation of dopamine in the brain, an extended feeling of pleasure, and an increase in reinforcing behavior ${ }^{66,67}$. The interactions between flavorings and nicotine in e-cigarettes may also be attributed to conditioned responses from previous experiences with food. That is, flavors that are currently used in e-cigarettes are often the same as those of rewarding foods (e.g., chocolate and pie) and become more palatable due to their associations with primary rewards (e.g., sugar) ${ }^{68,69}$. For example, menthol and licorice when established as a conditioned reinforcer by pairing them with sugar stimulate nicotine administration in rats 69. In summary, flavors stimulate palatability as well as reward from nicotine in e-cigarettes, and, hence, contribute to nicotine dependence not only through their physical properties (e.g., cooling) but also through their history of associative learning. 


\section{Regulation of e-cigarettes and e-liquids}

Regulation of product characteristics is important in order to reduce attractiveness, toxicity, and addictiveness of e-cigarettes and consequently the health risks associated with e-cigarette use ${ }^{70}$. Legislation on tobacco and related products on European (EU) level is laid down in the Tobacco Product Directive 2014/40/EU (TPD) ${ }^{71}$. The Netherlands, similar to other EU Member States, is required to adopt legislations from the TPD and can implement additional policy measures on national level in the Dutch Tobacco Act ${ }^{72,73}$.

In the EU, and thus in the Netherlands, regulation of e-cigarettes is mainly focused on environmental factors. For example, product accessibility is restricted through a minimum legal purchase age of 18 years, and there is a ban on advertisement and promotion of e-cigarettes and e-liquids. Furthermore, manufacturers and importers of e-cigarettes and e-liquids are required to notify Member State authorities of each product they intend to place on the market. Finally, on national level, a ban on using e-cigarettes in public places and a ban on displaying e-cigarettes in supermarkets became effective recently. Current EU regulations regarding e-cigarette product factors include: a maximum volume of the e-liquid container of $10 \mathrm{~mL}$, a maximum nicotine concentration of $20 \mathrm{mg} / \mathrm{mL}$, the need of an instruction leaflet including health warnings on e-cigarette and e-liquid packages, the obligation to use only ingredients of high purity, and a ban on using additives except nicotine that pose a risk to human health, are associated with reduced health risks or vitality (e.g., vitamins and caffeine), have coloring properties for emissions, or have reprotoxic properties ${ }^{71}$. Consequently, products with extremely high nicotine concentrations such as JUUL (59 mg/mL nicotine) are not allowed on the European market.

In Europe, characterizing flavors other than the one of tobacco are banned in combustible cigarettes and roll-your-own tobacco products since 2016, as flavored cigarettes stimulate smoking initiation among youth ${ }^{71}$. In the US, cigarettes with a flavor other than tobacco or menthol are banned ${ }^{74}$. In line with this, e-cigarette flavors could be regulated as well in order to decrease product attractiveness. However, currently, flavors in e-cigarettes are not regulated on European level, which causes e-liquids with appealing flavors to be widely available. The TPD states that EU Member States can individually decide to implement rules on flavors for e-cigarettes, taking "potential attractiveness of such products for young people and non-smokers" into account ${ }^{71}$. At the start of this research project, three European countries (Finland, Hungary, and Estonia) had banned or announced to ban e-liquids with a flavor other than tobacco ${ }^{75}$. More research is needed to inform the Dutch Ministry of Health, Welfare and Sport (VWS) and other regulatory agencies in and outside Europe on whether and how such flavor legislations should be extended towards e-cigarettes. 


\section{Thesis aim and outline}

Attractiveness of e-cigarette flavors may differ between individuals and populations (i.e., user groups). For example, general flavor preferences differ between youth and adults ${ }^{68}$. In addition, smokers may be attracted to different e-liquid flavors than non-smokers due to their associations with tobacco use. We hypothesized that e-liquid flavors could be identified that attract smokers but not youth and non-smokers. If regulatory authorities would allow only these flavors on the e-cigarette market, they may be able to facilitate smoking cessation, thereby decreasing the relative health risks for smokers, while preventing the use of e-cigarettes and associated health risks among young people and non-smokers. Therefore, this thesis aims to investigate the role of flavors in attractiveness of e-cigarettes with respect to different user groups. Within this thesis, several sub research questions are addressed (Table 1.1):

Table 1.1: Summary of the studies described in this thesis.

\begin{tabular}{|c|c|c|}
\hline Chapter & Aim & Method \\
\hline 2 & $\begin{array}{l}\text { Structuring e-liquid flavor names mentioned in literature, and } \\
\text { developing a tool for classification of e-liquids }\end{array}$ & Literature research \\
\hline 3 & Exploring e-liquid flavors available on the Dutch market & Database analysis \\
\hline 4 & $\begin{array}{l}\text { Exploring flavoring ingredients added to e-liquids on the Dutch } \\
\text { market, and predicting e-liquids' flavor category }\end{array}$ & $\begin{array}{l}\text { Database analysis and } \\
\text { machine learning }\end{array}$ \\
\hline 5 & $\begin{array}{l}\text { Identifying flavoring ingredients in e-liquids with different flavor } \\
\text { labels and comparing results between flavor categories }\end{array}$ & Chemical analysis \\
\hline 6 & $\begin{array}{l}\text { Investigating which e-liquid flavors are most interesting to never- } \\
\text { users and smokers, and mostly used by dual users and vapers in } \\
\text { the Netherlands }\end{array}$ & Survey research \\
\hline 7 & $\begin{array}{l}\text { Determining the correlation for hedonic assessment of e-liquid } \\
\text { flavors between smelling and vaping }\end{array}$ & Sensory research \\
\hline 8 & $\begin{array}{l}\text { Investigating hedonic assessment of tobacco and non-tobacco } \\
\text { e-liquid flavors in adolescent non-smokers, young adult non- } \\
\text { smokers, and adult smokers }\end{array}$ & Sensory research \\
\hline
\end{tabular}

\section{How can we provide structure to the large amount of available e-liquid flavors?}

In publications describing survey, laboratory, or human research on e-liquid flavors, various flavor names are mentioned. Often, specific e-liquid flavors are used to represent a broader flavor category. However, no consistent flavor language exists across literature, which makes a solid comparison of research results challenging. Food, alcohol, beverages, and fragrance industries commonly use flavor wheels as a flavor lexicon. Flavor wheels typically consist of an inner wheel with general descriptors (i.e., main categories), and an outer wheel with specific descriptors (i.e., subcategories). We aimed to identify and structure e-liquid flavor names and categories mentioned in literature, and develop a flavor wheel as a tool for consistent categorization of 
e-liquids based on their marketed flavor descriptions (Chapter 2). Such a lexicon could be used worldwide as a shared vocabulary for e-liquid flavors, which improves comparability of results across studies and provides a starting point for potential regulation of e-liquid flavors.

In order to take regulatory decisions on e-liquid flavors, it is important to determine what type of e-liquid flavors are available on the market. The market for e-liquids is huge, as endless combinations of flavoring ingredients can be added to e-liquids, and, as suggested by a study performed in the US, hundreds of different e-liquid brands are available ${ }^{76}$. However, data about the supply of e-liquids within Europe are lacking. As stated in the TPD, manufacturers are required to send information to regulating authorities of EU Member States about the products they plan to put on the market, including information about product ingredients and sales volumes ${ }^{71}$. This occurs via the European Common Entry Gate (EU-CEG) system, which is an electronic system that allows manufacturers to submit this information in a protected way ${ }^{77}$. This information can be used, for example, to signal new and emerging products, and to keep track of market trends. EU-CEG data about e-cigarettes and e-liquids have not been analyzed in any European country yet. We aimed to provide an overview of e-liquid flavors available on the Dutch market by classifying products into the categories of the e-liquid flavor wheel using information from the EU-CEG system ( $\underline{\text { Chapter 3 }}$ ).

\section{What are the most prevalent flavoring ingredients?}

Regulation of e-liquid flavors could focus on the flavor as mentioned on the product label, as perceived by consumers, or on the flavoring ingredients that result in the perceived flavor. For example, restricting the addition of popular flavorings and/or flavorings that are known to be toxic helps to reduce product appeal and health risks associated with e-cigarette use. In order to get insight into which flavorings manufacturers add to their e-liquids, information from the EU-CEG system can be used. We aimed to provide an overview of the flavoring ingredients, including their quantities, that manufacturers reported to have added to e-liquids marketed in the Netherlands (hapter 4$)$. In this study, we also aimed to develop an approach to predict e-liquids' marketed flavors (see Chapter 3 ) based on their flavoring ingredients. Such an automatic approach allows to create market overviews in a time-efficient manner, thereby facilitating comparative analyses between countries and at multiple points in time.

As information from the industry may not always be complete and correct, additional research is needed. Chemical analysis allows to determine compounds present in the e-liquid itself or in e-cigarette aerosol. Previous studies on e-liquid or aerosol compositions mostly focused on the determination of toxic and potentially toxic compounds. However, in terms of product appeal, data relating flavoring ingredients to e-liquids' marketed flavor descriptions are limited ${ }^{78}$. We aimed to identify flavoring ingredients in a large set of e-liquids with various flavor descriptions using chemical analysis, and to determine differences and similarities in e-liquid flavoring compositions between flavor categories (Chapter 5). Comparing flavoring compositions between flavor categories could inform policy makers about the type of e-liquid flavors (i.e., categories) that would be targeted in case particular flavoring ingredients would be restricted. 


\section{Which flavors do (potential) users prefer and like the most?}

After establishing which e-liquid flavors are available, it is important to determine which flavors are used the most and which flavors raise most interest among people who do not (yet) use the product. Different user groups should be defined, for which e-cigarette use may affect health in a different way: (1) people who have never smoked nor vaped (i.e., never-users) may initiate e-cigarette use in the future which negatively affects their health; (2) current smokers may switch towards e-cigarette use in the future and thereby improve their health; (3) people who concurrently smoke and vape (i.e., dual users) have not completely switched to vaping and still have major health risks; and (4) current exclusive vapers who are former smokers have successfully quit smoking and thus reduced their health risks. Whereas several surveys on flavor interest and use in these groups have been conducted in the US, such quantitative data are limited within Europe ${ }^{42,79-82}$. US data are only indicative for Europe and the Netherlands, as attractiveness is a concept defined by culture and e-cigarette use may differ between countries due to different preferences and legislations. Therefore, we aimed to investigate which e-cigarette flavors are most interesting to never-users and smokers, and which flavors are most popular among dual users and vapers in the Netherlands, using data from a survey (Chapter 6). In addition, Chapter 6 describes how individual factors related to vaping (e.g., knowledge, risk perception, and attitude towards vaping) differ between never-users and smokers who are and who are not interested in trying an e-liquid flavor.

Survey research is a rather indirect approach to investigating e-liquid flavor preferences, as it is based on respondents' mental representation and memory of flavor perception. A more direct approach is sensory research, which allows participants to actually taste or smell the product. Limited sensory research on tobacco and related products such as e-cigarettes has been performed. A few years ago, in assignment of the European Commission, a sensory method was developed to identify characterizing odors in combustible cigarettes and roll-your-own tobacco products using trained assessors (for enforcement of the ban on characterizing flavors) ${ }^{83,84}$. A few sensory studies on e-cigarettes have been performed in the US, for example investigating the relation between sweetness, bitterness, and harshness and (dis)liking of e-cigarettes using vaping experiments ${ }^{85-87}$. So far, no sensory study on e-cigarette flavors has been performed in Europe. However, this type of research is important in order to inform policy makers about which types of flavors are liked the most by which user group.

Sensory research on flavors can be performed by means of smelling and vaping. While vaping more closely represents real consumer behavior, smelling is easier, cheaper and associated with less ethical restrictions than vaping. We aimed to investigate whether smelling could be a successful alternative to vaping, by determining the correlation between smelling and vaping for the hedonic assessment of e-liquid flavors (Chapter 7). Subsequently, we aimed to determine which e-liquid flavors are attractive to specific user groups (Chapter 8). This was done by investigating the hedonic assessment of e-liquids with various tobacco and non-tobacco flavors, among adolescent non-smokers, young adult non-smokers, and adult smokers by smelling. Finally, this thesis contains a general discussion including a summary of the main findings, a discussion of the thesis topic and implications, methodological considerations, recommendations for future research, and main conclusions (hapter 9). 


\section{References}

1. World Health Organization. WHO global report on trends in prevalence of tobacco use 2000-2025, third edition. Geneva, 2019.

2. Farsalinos KE, Polosa R. Safety evaluation and risk assessment of electronic cigarettes as tobacco cigarette substitutes: a systematic review. Ther Adv Drug Saf. 2014;5(2):67-86.

3. Lik H. Google Patents. Electronic Atomization Cigarette. 2003; https://patents.google.com/ patent/ US20070267031. Accessed 15 June 2020.

4. Abrams DB. Promise and peril of e-cigarettes: can disruptive technology make cigarettes obsolete? JAMA. 2014;311(2):135-136.

5. Fagerstrom K, Etter JF, Unger JB. E-cigarettes: a disruptive technology that revolutionizes our field? Nicotine Tob Res. 2015;17(2):125-126.

6. Stimson GV, Thom B, Costall P. Disruptive innovations: the rise of the electronic cigarette. Int $J$ Drug Policy. 2014;25(4):653-655.

7. Philip Morris (PM). Invention record (Preliminary disclosure for consideration of patentability). 1990; https://www.industrydocuments.ucsf.edu/docs/\#id=tgcc0114. Accessed 16 June 2020.

8. Dutra LM, Grana R, Glantz SA. Philip Morris research on precursors to the modern e-cigarette since 1990. Tob Control. 2017;26(e2):e97-e105.

9. Mathur A, Dempsey OJ. Electronic cigarettes: a brief update. $J R$ Coll Physicians Edinb. 2018;48(4):346-351.

10. Goniewicz ML, Knysak J, Gawron M, et al. Levels of selected carcinogens and toxicants in vapour from electronic cigarettes. Tob Control. 2014;23(2):133-139.

11. Schmidt S. Vaper, Beware: The Unique Toxicological Profile of Electronic Cigarettes. Environ Health Perspect. 2020;128(5):52001.

12. Beard E, West R, Michie S, Brown J. Association between electronic cigarette use and changes in quit attempts, success of quit attempts, use of smoking cessation pharmacotherapy, and use of stop smoking services in England: time series analysis of population trends. BMJ. 2016;354:i4645.

13. Brown J, Beard E, Kotz D, Michie S, West R. Real-world effectiveness of e-cigarettes when used to aid smoking cessation: a cross-sectional population study. Addiction. 2014;109(9):1531-1540.

14. World Health Organization. WHO global report: mortaility attributable to tobacco. Geneva, 2012.

15. World Health Organization. Factsheet - Tobacco. 2020; https://www.who.int/news-room/fact-sheets/ detail/tobacco.

16. Soneji S, Barrington-Trimis JL, Wills TA, et al. Association Between Initial Use of e-Cigarettes and Subsequent Cigarette Smoking Among Adolescents and Young Adults: A Systematic Review and Meta-analysis. JAMA Pediatr. 2017;171(8):788-797.

17. Goniewicz ML, Smith DM, Edwards KC, et al. Comparison of Nicotine and Toxicant Exposure in Users of Electronic Cigarettes and Combustible Cigarettes. JAMA Netw Open. 2018;1(8):e185937.

18. Nationaal Expertisecentrum Tabaksontmoediging (Trimbos-instituut). Smoking in the Netherlands: key statistiscs for 2018. 2019; https://www.trimbos.nl/docs/edc3cfc9-f136-4246-9f34-9267046386ee. pdf.

19. Nationaal Expertisecentrum Tabaksontmoediging (Trimbos-instituut). Kerncijfers Roken 2019. 2020; https://www.trimbos.nl/docs/452115a7-4a93-4bd4-877a-ae4a7a4d1257.pdf.

20. Staatsblad van het Koninkrijk der Nederlanden. Decree of May 4, 2016 amending the decree implementing the Tobacco Act relating to the implementation of Directive 2014/40/EU on 
the manufacture, presentation and sale of tobacco and related products. 2016; https://www. tobaccocontrollaws.org/files/live/Netherlands/Netherlands\%20-\%20Decree $\% 20$ of $\% 20 \mathrm{May} \% 20$ 4\%2C\%202016\%20\%20-\%20national.pdf.

21. Stevens G, van Dorsselaer S, Boer M, et al. HBSC 2017: Gezondheid en welzijn van jongeren in Nederland. 2018; https://hbsc-nederland.nl/wp-content/uploads/2018/09/Rapport-HBSC-2017.pdf.

22. Cullen KA, Ambrose BK, Gentzke AS, Apelberg BJ, Jamal A, King BA. Notes from the Field: Use of Electronic Cigarettes and Any Tobacco Product Among Middle and High School Students - United States, 2011-2018. MMWR Morb Mortal Wkly Rep. 2018;67(45):1276-1277.

23. King BA, Gammon DG, Marynak KL, Rogers T. Electronic Cigarette Sales in the United States, 2013-2017. JAMA. 2018;320(13):1379-1380.

24. Cullen KA, Gentzke AS, Sawdey MD, et al. e-Cigarette Use Among Youth in the United States, 2019. JAMA. 2019;322(21):2095-2103.

25. US Department of Health and Human Services. Surgeon General's advisory on e-cigarette use among youth. 2018; https://e-cigarettes.surgeongeneral.gov/documents/surgeon-generals-advisoryon-e-cigarette-use-among-youth-2018.pdf. Accessed 18 June 2020.

26. US Food and Drug Administration (FDA). Statement from FDA Commissioner Scott Gottlieb, M.D., on new steps to address epidemic of youth e-cigarette use [press release]. Silver Spring, MD, September 11, 2018.

27. Pacek LR, Wiley JL, McClernon FJ. A Conceptual Framework for Understanding Multiple Tobacco Product Use and the Impact of Regulatory Action. Nicotine Tob Res. 2019;21(3):268-277.

28. Shepherd R. Does Taste Determine Consumption? Understanding the Psychology of Food Choice. In: Frewer LJ, Risvik E, Schifferstein H, eds. Food, People and Society. Berlin, Heidelberg: Springer Berlin Heidelberg; 2001:117-130.

29. Baweja R, Curci KM, Yingst J, et al. Views of Experienced Electronic Cigarette Users. Addict Res Theory. 2016;24(1):80-88.

30. Kistler CE, Crutchfield TM, Sutfin EL, et al. Consumers' Preferences for Electronic Nicotine Delivery System Product Features: A Structured Content Analysis. Int J Environ Res Public Health. 2017;14(6).

31. Berg CJ. Preferred flavors and reasons for e-cigarette use and discontinued use among never, current, and former smokers. Int J Public Health. 2016;61(2):225-236.

32. Romijnders K, van Osch L, de Vries H, Talhout R. Perceptions and Reasons Regarding E-Cigarette Use among Users and Non-Users: A Narrative Literature Review. Int $J$ Environ Res Public Health. 2018;15(6).

33. Landry RL, Groom AL, Vu TT, et al. The role of flavors in vaping initiation and satisfaction among U.S. adults. Addict Behav. 2019;99:106077.

34. Cheney MK, Gowin M, Wann TF. Electronic Cigarette Use in Straight-to-Work Young Adults. Am J Health Behav. 2016;40(2):268-279.

35. Patel D, Davis KC, Cox S, et al. Reasons for current E-cigarette use among U.S. adults. Prev Med. 2016;93:14-20.

36. Hilton S, Weishaar H, Sweeting H, Trevisan F, Katikireddi SV. E-cigarettes, a safer alternative for teenagers? A UK focus group study of teenagers' views. BMJ Open. 2016;6(11):e013271.

37. Bold KW, Kong G, Cavallo DA, Camenga DR, Krishnan-Sarin S. Reasons for Trying E-cigarettes and Risk of Continued Use. Pediatrics. 2016;138(3). 
38. Kong G, Morean ME, Cavallo DA, Camenga DR, Krishnan-Sarin S. Reasons for Electronic Cigarette Experimentation and Discontinuation Among Adolescents and Young Adults. Nicotine Tob Res. 2015; 17(7):847-854.

39. Ambrose BK, Day HR, Rostron B, et al. Flavored Tobacco Product Use Among US Youth Aged 1217 Years, 2013-2014. JAMA. 2015;314(17):1871-1873.

40. Rijksinstituut voor Volksgezondheid en Milieu (RIVM). E-sigaret aantrekkelijkheid voor rokers en niet-rokers. 2018; https://www.rivm.nl/sites/default/files/2018-11/Informatiebrochure\%20 aantrekkelijkheid\%20e-sigaretten\%20definitief.pdf.

41. Pepper JK, Ribisl KM, Brewer NT. Adolescents' interest in trying flavoured e-cigarettes. Tob Control. 2016;25(Suppl 2):ii62-ii66.

42. Ford A, MacKintosh AM, Bauld L, Moodie C, Hastings G. Adolescents' responses to the promotion and flavouring of e-cigarettes. Int J Public Health. 2016;61(2):215-224.

43. Strombotne K, Buckell J, Sindelar JL. Do JUUL and e-cigarette flavours change risk perceptions of adolescents? Evidence from a national survey. Tob Control. 2020:tobaccocontrol-2019-055394.

44. Leigh NJ, Lawton RI, Hershberger PA, Goniewicz ML. Flavourings significantly affect inhalation toxicity of aerosol generated from electronic nicotine delivery systems (ENDS). Tob Control. 2016;25(Suppl 2):ii81-ii87.

45. Lerner CA, Sundar IK, Yao H, et al. Vapors produced by electronic cigarettes and e-juices with flavorings induce toxicity, oxidative stress, and inflammatory response in lung epithelial cells and in mouse lung. PLoS One. 2015;10(2):e0116732.

46. Rankin GD, Wingfors H, Uski O, et al. The toxic potential of a fourth-generation E-cigarette on human lung cell lines and tissue explants. J Appl Toxicol. 2019;39(8):1143-1154.

47. Kuntic M, Oelze M, Steven S, et al. Short-term e-cigarette vapour exposure causes vascular oxidative stress and dysfunction: evidence for a close connection to brain damage and a key role of the phagocytic NADPH oxidase (NOX-2). Eur Heart J. 2020;41(26):2472-2483.

48. 4Greene RM, Pisano MM. Developmental toxicity of e-cigarette aerosols. Birth Defects Res. 2019;111(17):1294-1301.

49. Hutzler C, Paschke M, Kruschinski S, Henkler F, Hahn J, Luch A. Chemical hazards present in liquids and vapors of electronic cigarettes. Arch Toxicol. 2014;88(7):1295-1308.

50. Tierney PA, Karpinski CD, Brown JE, Luo W, Pankow JF. Flavour chemicals in electronic cigarette fluids. Tob Control. 2016;25(e1):e10-15.

51. Kaur G, Muthumalage T, Rahman I. Mechanisms of toxicity and biomarkers of flavoring and flavor enhancing chemicals in emerging tobacco and non-tobacco products. Toxicol Lett. 2018;288:143155.

52. Fagan P, Pokhrel P, Herzog TA, et al. Sugar and Aldehyde Content in Flavored Electronic Cigarette Liquids. Nicotine Tob Res. 2018;20(8):985-992.

53. Khlystov A, Samburova V. Flavoring Compounds Dominate Toxic Aldehyde Production during E-Cigarette Vaping. Environ Sci Technol. 2016;50(23):13080-13085.

54. Polosa R, O’Leary R, Tashkin D, Emma R, Caruso M. The effect of e-cigarette aerosol emissions on respiratory health: a narrative review. Expert Rev Respir Med. 2019;13(9):899-915.

55. DeVito EE, Krishnan-Sarin S. E-cigarettes: Impact of E-Liquid Components and Device Characteristics on Nicotine Exposure. Curr Neuropharmacol. 2018;16(4):438-459.

56. De Biasi M, Dani JA. Reward, addiction, withdrawal to nicotine. Annu Rev Neurosci. 2011;34:105- 
130.

57. England LJ, Bunnell RE, Pechacek TF, Tong VT, McAfee TA. Nicotine and the Developing Human: A Neglected Element in the Electronic Cigarette Debate. Am J Prev Med. 2015;49(2):286-293.

58. Berry KM, Fetterman JL, Benjamin EJ, et al. Association of Electronic Cigarette Use With Subsequent Initiation of Tobacco Cigarettes in US Youths. JAMA Netw Open. 2019;2(2):e187794.

59. Owotomo O, Stritzel H, McCabe SE, Boyd CJ, Maslowsky J. Smoking Intention and Progression From E-Cigarette Use to Cigarette Smoking. Pediatrics. 2020:e2020002881.

60. Audrain-McGovern J, Strasser AA, Wileyto EP. The impact of flavoring on the rewarding and reinforcing value of e-cigarettes with nicotine among young adult smokers. Drug Alcohol Depend. 2016;166:263-267.

61. Kroemer NB, Veldhuizen MG, Delvy R, Patel BP, O’Malley SS, Small DM. Sweet taste potentiates the reinforcing effects of e-cigarettes. Eur Neuropsychopharmacol. 2018;28(10):1089-1102.

62. van de Nobelen S, Kienhuis AS, Talhout R. An Inventory of Methods for the Assessment of Additive Increased Addictiveness of Tobacco Products. Nicotine Tob Res. 2016;18(7):1546-1555.

63. Cooper SY, Akers AT, Henderson BJ. Green Apple e-Cigarette Flavorant Farnesene Triggers RewardRelated Behavior by Promoting High-Sensitivity nAChRs in the Ventral Tegmental Area. eNeuro. 2020;7(4).

64. Benowitz NL, Herrera B, Jacob P, 3rd. Mentholated cigarette smoking inhibits nicotine metabolism. J Pharmacol Exp Ther. 2004;310(3):1208-1215.

65. Winters BR, Kochar TK, Clapp PW, Jaspers I, Madden MC. Impact of E-Cigarette Liquid Flavoring Agents on Activity of Microsomal Recombinant CYP2A6, the Primary Nicotine-Metabolizing Enzyme. Chem Res Toxicol. 2020;33(7):1689-1697.

66. Truman P, Stanfill S, Heydari A, Silver E, Fowles J. Monoamine oxidase inhibitory activity of flavoured e-cigarette liquids. Neurotoxicology. 2019;75:123-128.

67. He X, Chen YY, Shi JB, et al. New coumarin derivatives: design, synthesis and use as inhibitors of hMAO. Bioorg Med Chem. 2014;22(14):3732-3738.

68. Hoffman AC, Salgado RV, Dresler C, Faller RW, Bartlett C. Flavour preferences in youth versus adults: a review. Tob Control. 2016;25(Suppl 2):ii32-ii39.

69. Palmatier MI, Smith AL, Odineal EM, Williams EA, Sheppard AB, Bradley CA. Nicotine SelfAdministration With Tobacco Flavor Additives in Male Rats. Nicotine Tob Res. 2020;22(2):224-231.

70. World Health Organization. Partial guidelines for implementation of Articles 9 and 10 of the WHO Framework Convention on Tobacco Control: Regulation of the contents of tobacco products and of tobacco product disclosures. 2012; https:/www.who.int/fctc/guidelines/Decisions9and10.pdf.

71. The European Parliament and The Council of the European Union. Directive 2014/40/EU on the approximation of the laws, regulations and administrative provisions of the Member States concerning the manufacture, presentation and sale of tobacco and related products and repealing Directive 2001/37/EC. Vol 2014/40/EU: Official Journal of the European Union; 2014:127.

72. Eerste Kamer der Staten-Generaal. 34 234: Wijziging van de Tabakswet ter implementatie van Richtlijn 2014/40/EU, inzake de productie, de presentatie en de verkoop van tabaks- en aanverwante producten. 2016; https://www.senaat.eu/9370000/1/j9vvkfvj6b325az/vk19qh14wfyd.

73. Ministry of Health Welfare and Sports (the Netherlands). Tabaks- en rookwarenwet (Dutch Tobacco Act). 2020; https://wetten.overheid.nl/BWBR0004302/2020-07-01.

74. The Senate and House of Representatives of the United States of America in Congress. Public Law 
111-31: Family Smoking Prevention and Tobacco Control and Federal Retirement Reform. In: $111^{\text {th }}$ Congress; 2009:1776-1858.

75. Gruszczynski L. The regulation of e-cigarettes: international, European and national challenges. Cheltenham (UK) and Northampton (USA): Edward Elgar Publishing; 2019:159.

76. Zhu SH, Sun JY, Bonnevie E, et al. Four hundred and sixty brands of e-cigarettes and counting: implications for product regulation. Tob Control. 2014;23 Suppl 3:iii3-9.

77. European Commission. EU Common Entry Gate (EU-CEG). 2016; https://ec.europa.eu/health/ euceg/introduction_en.

78. Aszyk J, Kubica P, Wozniak MK, Namiesnik J, Wasik A, Kot-Wasik A. Evaluation of flavour profiles in e-cigarette refill solutions using gas chromatography-tandem mass spectrometry. J Chromatogr A. 2018;1547:86-98.

79. Dawkins L, Turner J, Roberts A, Soar K. 'Vaping' profiles and preferences: an online survey of electronic cigarette users. Addiction. 2013;108(6):1115-1125.

80. Laverty AA, Vardavas CI, Filippidis FT. Design and marketing features influencing choice of e-cigarettes and tobacco in the EU. Eur J Public Health. 2016;26(5):838-841.

81. Rijksinstituut voor Volksgezondheid en Milieu (RIVM). De gezondheidsrisico's van e-sigaret gebruik. 2015; https://www.rivm.nl/bibliotheek/rapporten/2014-0143.pdf.

82. Farsalinos KE, Romagna G, Tsiapras D, Kyrzopoulos S, Spyrou A, Voudris V. Impact of flavour variability on electronic cigarette use experience: An internet survey. Int J Environ Res Public Health. 2013;10(12):7272-7282.

83. HETOC Consortium. Mapping of best practices and development of testing methods and procedures for identification of characterising flavours in tobacco products. 2016; http://ec.europa.eu/health/ tobacco/docs/hetoc_frep_en.pdf.

84. Krusemann EJZ, Lasschuijt MP, de Graaf C, et al. Sensory analysis of characterising flavours: evaluating tobacco product odours using an expert panel. Tob Control. 2019;28(2):152-160.

85. Kim H, Lim J, Buehler SS, et al. Role of sweet and other flavours in liking and disliking of electronic cigarettes. Tob Control. 2016;25(Suppl 2):ii55-ii61.

86. Pullicin AJ, Kim H, Brinkman MC, Buehler SS, Clark PI, Lim J. Impacts of Nicotine and Flavoring on the Sensory Perception of E-Cigarette Aerosol. Nicotine Tob Res. 2020;22(5):806-813.

87. Mead EL, Duffy V, Oncken C, Litt MD. E-cigarette palatability in smokers as a function of flavorings, nicotine content and propylthiouracil (PROP) taster phenotype. Addict Behav. 2019;91:37-44. 



\section{CHAPTER 2}

\section{An e-liquid flavor wheel: A shared vocabulary \\ based on systematically reviewing e-liquid flavor \\ classifications in literature}

Erna J.Z. Krüsemann

Sanne Boesveldt

Kees de Graaf

Reinskje Talhout

Published in Nicotine \& Tobacco Research. 2019;21(10):1310-1319.

https://doi.org/10.1093/ntr/nty101. 


\begin{abstract}
Introduction: E-liquids are available in a high variety of flavors. A systematic classification of e-liquid flavors is necessary to increase comparability of research results. In the food, alcohol and fragrance industry, flavors are classified using flavor wheels. We systematically reviewed literature on flavors related to e-cigarette use, to investigate how e-liquid flavors have been classified in research, and propose an e-liquid flavor wheel to classify e-liquids based on marketing descriptions. Methods: The search was conducted in May 2017 using PubMed and Embase databases. Keywords included terms associated with e-cigarettes, flavors, liking, learning, and wanting in articles. Results were independently screened and reviewed. Flavor categories used in the articles reviewed were extracted. Results: Searches yielded 386 unique articles of which 28 were included. Forty-three main flavor categories were reported in these articles (e.g., tobacco, menthol, mint, fruit, bakery/dessert, alcohol, nuts, spice, candy, coffee/tea, beverages, chocolate, sweet flavors, vanilla, unflavored). Flavor classifications of e-liquids in literature showed similarities and differences across studies. Our proposed e-liquid flavor wheel contains 13 main categories and 90 subcategories, which summarize flavor categories from literature to find a shared vocabulary. For classification of e-liquids using our flavor wheel, marketing descriptions should be used. Conclusions: We have proposed a flavor wheel for classification of e-liquids. Further research is needed to test the flavor wheels' empirical value. Consistently classifying e-liquid flavors using our flavor wheel in research (e.g., experimental, marketing, or qualitative studies) minimizes interpretation differences and increases comparability of results. Implications: We reviewed e-liquid flavors and flavor categories used in research. A large variation in the naming of flavor categories was found and e-liquid flavors were not consistently classified. We developed an e-liquid flavor wheel and provided a guideline for systematic classification of e-liquids based on marketing descriptions. Our flavor wheel summarizes e-liquid flavors and categories used in literature in order to create a shared vocabulary. Applying our flavor wheel in research on e-liquids will improve data interpretation, increase comparability across studies, and support policy makers in developing rules for regulation of e-liquid flavors.
\end{abstract}




\section{Introduction}

Electronic cigarettes (e-cigarettes) vaporize e-liquids, which consist of a propylene glycol and glycerol base, and a varying amount of nicotine and flavorings ${ }^{1}$. Flavorings are the flavor molecules present in e-liquids that contribute to the perceived flavor, whereas we refer to flavors as the combined sensations of taste and smell of e-liquids from a particular brand. The number of available e-liquid flavors exceeded 7500 in 2014 and is still increasing ${ }^{2}$. These flavors increase sensory appeal of the e-liquid ${ }^{3}$. Increasing attractiveness of e-liquid flavors could stimulate smokers to use an e-cigarette as alternative for regular cigarettes, as non-tobacco and nonmenthol flavors are associated with higher rates of smoking cessation ${ }^{4-6}$. On the other hand, it is well established that flavors in tobacco products generally attract adolescents and youth ${ }^{7-10}$. Flavor preferences may also play an important role in e-cigarette use among adolescents ${ }^{11}$. Especially non-tobacco e-liquid flavors are attractive to non-smoking youth, thereby stimulating use and nicotine consumption ${ }^{12-14}$.

Nicotine-containing e-liquids have a stimulating effect on the reward system within the brain, which is implicated in the development of addiction ${ }^{15}$. The core psychological components of reward are liking, learning and wanting ${ }^{16}$. Whereas flavors are added to increase product liking, addictive substances such as nicotine play a role in motivation and influence the reward system through mechanisms of learning and wanting. Considering existing literature, research has mostly focused on the role of flavors in liking of e-cigarettes, providing insight in e-cigarette use and preferences. For instance, a review of Huang et al. ${ }^{17}$ showed that most e-cigarette users prefer non-traditional flavors such as fruit and sweet flavors compared to traditional flavors such as tobacco or menthol. In addition, a recent study showed that adolescents predominantly prefer fruit, candy/dessert, and vanilla, whereas the most preferred flavors among adults are non-sweet e-cigarette flavors such as fruit, tobacco, and menthol/mint ${ }^{11}$. For regulation purposes, it is important to understand how flavor liking differs among different consumer groups, for example, adult tobacco smokers and non-smoking adolescents or youth. However, as the variety of available e-liquid flavors increases and more and more research is being conducted, a systematic way of flavor classification is needed in order to increase comparability of results and facilitate data interpretation among researchers and policy makers.

Flavor wheels have been developed as a tool to consistently classify flavors and/or aromas in the food, alcohol, and fragrance industries. A flavor wheel visually represents a shared vocabulary of flavor attributes that are classified into general categories. For instance, Noble et al. ${ }^{18}$ developed a wine aroma wheel in 1984 containing 12 main categories such as fruity, vegetative, nutty, earthy, chemical, floral, and spicy, and uses sub attributes for specification. Similarly, flavor wheels have been developed for other alcoholic beverages (e.g., beer and whiskey) ${ }^{19,20}$, food products (e.g., chocolate, coffee, olive oil, and cheese) ${ }^{21-24}$, and for fragrances ${ }^{25}$. Regarding tobacco products, the industry has created a cigar flavor wheel that consists of 8 main categories and 52 subcategories ${ }^{26}$. These flavor wheels are used as a common vocabulary within industries and science, for instance as a tool used by consumer or expert panels to assess flavor attributes.

While the number of unique e-cigarette flavors is increasing, no flavor wheel for e-liquids currently exists. We have reviewed e-liquid flavor classification in existing literature and propose a flavor wheel to systematically classify e-liquid flavors. The importance of developing 
a systematic flavor classification for e-liquids was previously mentioned by Yingst et al. ${ }^{27}$, who conducted a survey about participants' favorite e-liquid flavor. The researchers used the participants' responses to develop a list of flavor categories and guidelines for classification of e-liquid flavors. Flavor classifications may differ across study disciplines, as individuals interpret e-liquid brand names and marketing descriptions in a different way. We therefore reviewed existing literature (including the publication of Yingst et al. ${ }^{27}$ ) to investigate which classifications and terminology researchers have used in order to find a commonly agreed flavor vocabulary. To develop a shared vocabulary, we propose an e-liquid flavor wheel that summarizes flavor categories from literature. The flavor wheel could be applied to multiple research disciplines, for instance, to investigate liking of particular flavor categories among different consumer groups. Applying our flavor wheel for e-liquids will facilitate communication among and between researchers, consumers, and policy makers, which will improve data interpretation and increase comparability of results across studies. 


\section{Methods}

\section{Data sources and search}

Our search strategy aimed to identify peer-reviewed journal articles in which flavors are investigated in relation to e-cigarette use and preferences. The strategy was developed with the assistance of an experienced librarian with expertise in conducting and documenting literature searches. The search was conducted in May 2017 using PubMed and Embase databases. The search was updated to include current literature up to January 2018. Keywords included terms to capture concepts associated with e-cigarettes, flavors, liking, learning, and wanting. Articles published between the year of 1990 and the search date were included. As an example, the complete search strategy for the PubMed database is added in Appendix Table A2.1.

\section{Study selection and exclusion criteria}

Retrieved articles were screened, duplicates were eliminated, and remaining citations were organized in EndNote (Clarivate Analytics, Philadelphia, PA) following Preferred Reporting Items for Systematic Reviews and Meta-Analyses (PRISMA) guidelines (Figure 2.1). First, two authors (EK and RT) created and agreed on a list of exclusion criteria, and independently screened a random sample of 66 titles and abstracts, blinded to authors and journal titles, for interrater reliability ${ }^{28}$. The Cohen's kappa reached 0.92 , which is considered an almost perfect level of agreement ${ }^{29}$. Second, the same two authors independently screened the total set of titles and abstracts, blinded to authors and journal titles ${ }^{30}$. Data were compiled into an Excel workbook and consensus was reached on titles and abstracts that the authors evaluated in a different way ${ }^{31}$. Articles were excluded (Figure 2.1) when e-cigarettes were not the research topic $(\mathrm{n}=194)$. In addition, articles about toxicity, health, or health risks $(n=59)$; chemical-analytical research articles on liquid composition $(\mathrm{n}=17)$; articles of which the title and abstract did not mention the word flavor or a specific flavor $(n=12)$; or review articles $(n=6)$ were excluded. In the third phase, the first author (EK) reviewed full-text articles to determine final eligibility. Articles were excluded if e-cigarettes were not the research topic $(n=11)$; the article described toxicology or health risks $(n=21)$ or chemical composition $(n=3)$; flavors were not the main research topic $(n=9)$; the article was a literature review $(n=3)$; the topic was legislation $(n=3)$; the article was non-peer-reviewed $(n=12)$; data were incomplete or insufficient $(n=5)$; or if the article did not use e-liquid flavor categories $(n=6)$. As we were interested in flavor classifications only to provide a broad overview of interpretations of researchers in order to develop a common flavor vocabulary, no articles were excluded based on quality (internal or external validity). Articles encountered via citation tracking that were considered eligible for inclusion were reviewed using the previously mentioned exclusion criteria $(n=2)$. 
521 records identified from

all sources

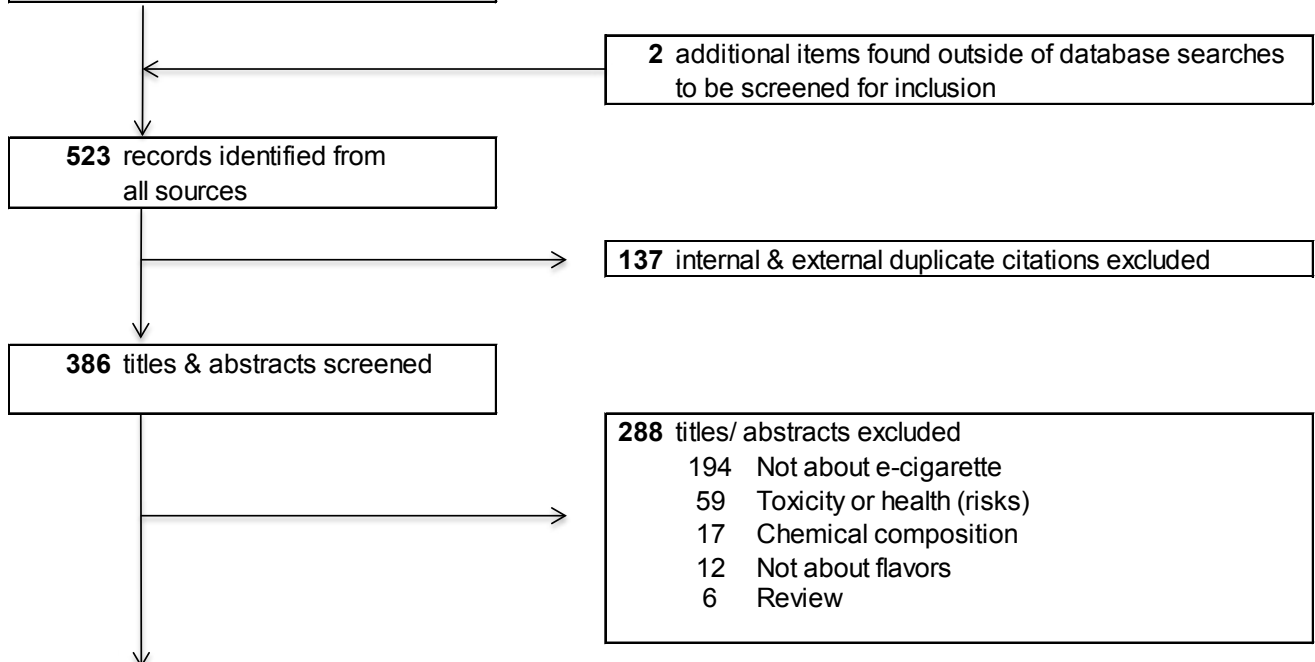

98 full text records to be reviewed

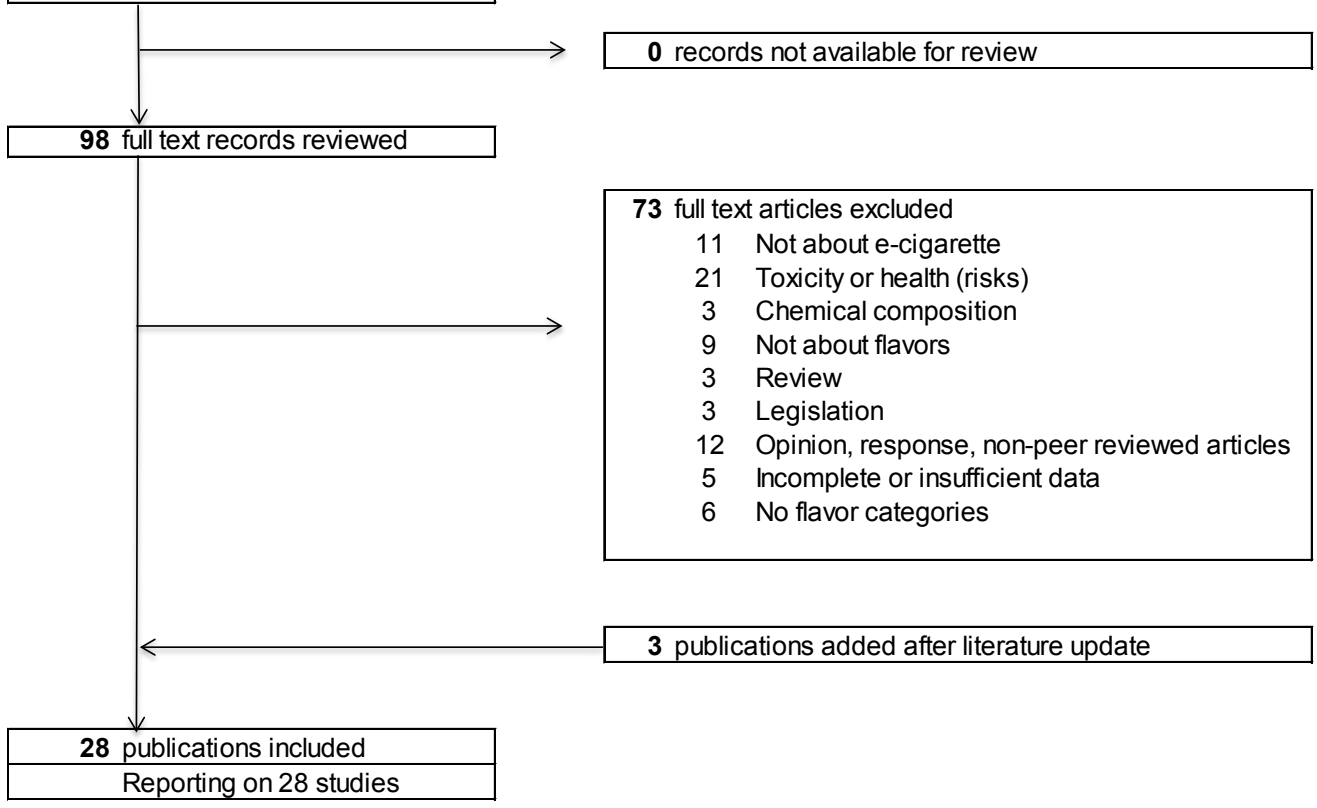

Figure 2.1: PRISMA flowchart. Articles were retrieved from PubMed and Embase databases $(\mathrm{n}=521)$ and via citation tracking $(n=2)$. Articles published between the year of 1990 and the search date (May 2017; updated in January 2018) were included. 


\section{Data extraction and synthesis}

Included articles $(\mathrm{n}=28)$ were analyzed by the first author using a data extraction table. The articles included have used a certain classification of e-cigarette flavors for data reduction, either to explain which flavors they used (e.g., for experimental setups) or to categorize their results (e.g., for surveys). For instance, Tackett et al. ${ }^{6}$ conducted a survey in which e-cigarette flavors were represented by six categories: fruity, bakery/dessert, tobacco blends, mint/menthol, candy/ nuts and coffee. From each article, the flavor categories used in the study design were extracted. A distinction was made between main flavor categories (e.g., fruit or spice) and subcategories (specific e-liquid flavors that represent these categories, e.g., lemon or cinnamon). For instance, the answer options of survey questions about consumers' preferred e-liquid flavor (e.g., "fruit" or "candy") were main flavor categories, while the examples that researchers used to explain or specify these categories (e.g., "e.g., cherry, watermelon, kiwi" or "e.g., bubble gum") were considered specific e-liquid flavors that represent the main flavor categories. Another example: if researchers compared sweet flavors with non-sweet flavors, we considered "sweet" and "nonsweet" as the main flavor categories. The examples that researchers use as specification of these main categories were considered subcategories (e.g., "chocolate" or "vanilla" as subcategory of sweet flavors, and "tobacco" or "menthol" as subcategory of non-sweet flavors).

Some of the main flavor categories or subcategories identified from literature were used in more than one article; hence, prevalence of each flavor category was determined. Results were summarized in a table that shows each main flavor category and associated subcategories (i.e., flavor examples of the main categories) used in the articles reviewed.

\section{Generation of the flavor wheel}

The flavor categories extracted from literature served as a basis for our flavor wheel. Similar flavor categories were combined into one category. The name of this category was based on the name that was predominantly used in the articles reviewed (i.e., based on prevalence numbers of the flavor categories). Resulting categories formed the inner layer of the flavor wheel.

The specific e-liquids that were used in literature as examples of the main categories were considered representative examples of the main categories. Therefore, each of the specific e-liquid flavors mentioned in literature was used as subcategory in the outer layer of the flavor wheel. Brand names were excluded to solely include generic and generally known category names. Subcategories were sorted to be mutually exclusive; hence, each of the specific flavors used as example of a main category was associated to only one of the main categories. Classification of subcategories within main categories was based on classifications in articles reviewed, and flavor wheels from the food, alcohol, and fragrance industries $19,20,22,24-26,32$. 


\section{Results}

Database searches and citation tracking yielded 386 unique articles of which 25 met all inclusion criteria. A literature search update led to three additional eligible articles, resulting in a total inclusion of 28 publications. Most studies were conducted in the United States $(n=21)$. Other study locations were UK $(\mathrm{n}=3)$, Canada $(\mathrm{n}=2)$, Greece/Italy $(\mathrm{n}=1)$, and China/United States $(\mathrm{n}=1)$. An overview of study characteristics is added in Appendix Table A2.2.

Analysis of flavor classifications used in the articles reviewed resulted in 43 unique main flavor categories, which are shown, including their prevalence across articles, in Table 2.1. Clustering similar categories resulted in 13 clusters of tobacco-, menthol-, fruit-, dessert-, alcohol-, nut-, spices-, candy-, coffee/tea-, beverages-, and sweet-like flavors, unflavored e-liquids, and unspecified flavors. The third column of Table 2.1 describes specific flavors mentioned as example of one of the main categories. For instance, Tackett et al. ${ }^{6}$ mentioned strawberry and blueberry as examples of their fruity category, and cotton candy, SweetTart, hazelnut, and almond as examples of the candy/nuts category. We indicated prevalence of these specific flavors when a flavor was mentioned as example of a particular category in more than one article.

The number of flavor categories used in the included articles varied from 1 to 11 . For instance, Vasiljevic et al. ${ }^{33}$ conducted an experimental study with candy-flavored e-cigarettes only, whereas the survey of Yingst et al. ${ }^{27}$ distinguished between 11 categories, being tobacco, menthol/mint, fruit, dessert/sweets, alcohol, nuts/spices, candy, coffee/tea, other beverages, unflavored, and don't know/other flavors. Considering flavor categories and classifications in literature, Table 2.1 shows that some of the flavor categories were used in more than one article. However, clustering similar categories shows that different category names were used to express the same type of flavors. 
Table 2.1: Main flavor categories used in the articles reviewed (first column), prevalence across articles (second column), and the e-liquid flavors mentioned as an example of these categories (third column).

\begin{tabular}{|c|c|c|c|}
\hline $\begin{array}{l}\text { Main flavor categories } \\
\text { from literature }(n=43)\end{array}$ & Prevalence & E-liquid flavors mentioned as example & References \\
\hline Tobacco & 19 & tobacco $(n=3)$, menthol & $4-6,11-13,27,34-45$ \\
\hline Tobacco or menthol & 2 & tobacco, menthol & 46,47 \\
\hline Menthol & 10 & menthol $(n=2)$, mint $(n=2)$, menthol tobacco & $\begin{array}{l}4,11,35,39- \\
42,44,45,48\end{array}$ \\
\hline Menthol/mint & 7 & menthol, mint, peppermint & $5,6,12,27,34,36,48$ \\
\hline Mint & 2 & & 11,49 \\
\hline Nuts & 3 & nuts $(\mathrm{n}=2)$ & $5,35,45$ \\
\hline Nuts/spices & 1 & almond, cinnamon, peanut butter, pecan & 27 \\
\hline Seasonings & 2 & cinnamon $(n=2)$, pepper $(n=2)$ & 35,45 \\
\hline Spice & 4 & cinnamon $(n=2)$, clove, nutmeg & $11,12,44,49$ \\
\hline Coffee & 6 & cappuccino, espresso, latte & $4,6,11,36,37,44$ \\
\hline Coffee/alcohol & 2 & & 12,49 \\
\hline Coffee/tea & 2 & cappuccino, coffee, espresso, tea & 27,34 \\
\hline Alcohol & 6 & $\begin{array}{l}\text { absinthe, absolut, bourbon, champagne, } \\
\text { (strawberry) daiquiri, mojitos, piña colada, rum, } \\
\text { scotch }\end{array}$ & $11,27,34,36,42,44$ \\
\hline Beverages & 3 & $\begin{array}{l}\text { coffee }(n=3), \text { alcoholic drinks, soda, tea }(n=2) \text {, } \\
\text { wine }(n=2)\end{array}$ & $35,45,50$ \\
\hline Beverages/drinks & 1 & & 5 \\
\hline Other beverages & 1 & energy drinks, lemonades, sodas & 27 \\
\hline Cherry & 1 & & 4 \\
\hline Fruit & 18 & $\begin{array}{l}\text { cherry }(n=7), \text { strawberry }(n=7) \text {, apple }(n=4) \text {, } \\
\text { blueberry }(n=4) \text {, mango }(n=3) \text {, orange }(n=3) \text {, } \\
\text { peach }(n=3) \text {, watermelon }(n=3) \text {, banana }(n= \\
\text { 2), berry }(n=2) \text {, lemon }(n=2) \text {, pomegranate }(n= \\
\text { 2), raspberry }(n=2) \text {, coconut, grape, green apple, } \\
\text { lime, pear, plum }\end{array}$ & $\begin{array}{l}5,6,11-13,27,34- \\
37,40,42,44,45,47,49- \\
51\end{array}$ \\
\hline Bakery/dessert & 1 & & 6 \\
\hline Cream & 2 & $\begin{array}{l}\text { cake }(n=2) \text {, chocolate }(n=2) \text {, cookie }(n=2) \text {, } \\
\text { custard }(n=2) \text {, milk }(n=2) \text {, vanilla }(n=2) \text {, } \\
\text { butter, cheese, cream }\end{array}$ & 35,45 \\
\hline Dessert & 1 & chocolate & 51 \\
\hline Dessert/sweets & 1 & $\begin{array}{l}\text { cakes, cereals, chocolate, donuts, ice cream, quick } \\
\text { breads, vanilla, waffles }\end{array}$ & 27 \\
\hline Food/dessert/spice & 1 & banana foster, coffee, peaches, vanilla & 47 \\
\hline Candy & 7 & $\begin{array}{l}\text { gummy bears }(n=3) \text {, licorice }(n=2) \text {, bubble } \\
\text { gum, chocolate, Swedish fish, SweetTarts, vanilla }\end{array}$ & $27,33,34,42,44,46,49$ \\
\hline Candy or dessert & 2 & $\begin{array}{l}\text { chocolate }(\mathrm{n}=2) \text {, apple pie, gummy bear, Jolly } \\
\text { Rancher, vanilla }\end{array}$ & 11,12 \\
\hline Candy/nuts & 1 & almond, cotton candy, hazelnut, SweetTart & 6 \\
\hline $\begin{array}{l}\text { Caramel, vanilla, } \\
\text { chocolate or cream }\end{array}$ & 1 & & 34 \\
\hline Chocolate & 1 & chocolate & 40 \\
\hline
\end{tabular}




\begin{tabular}{|c|c|c|c|}
\hline Chocolate/sweet & 1 & & 36 \\
\hline Sweet & 7 & $\begin{array}{l}\text { candy }(\mathrm{n}=3) \text {, honey }(\mathrm{n}=2) \text {, blackberry, candy } \\
\text { floss, caramel, chocolate, cola, cotton candy, } \\
\text { desserts, peach, sweet lemon tea, vanilla, } \\
\text { watermelon }\end{array}$ & $3,5,35,37,39,45,50$ \\
\hline Vanilla & 2 & & 11,36 \\
\hline Flavorless & 2 & & 3,36 \\
\hline Unflavored & 5 & PG/VG base only $(n=2)$ & $12,27,40,48,51$ \\
\hline Combination of flavors & 2 & $\begin{array}{l}\text { blueberry champagne, bubble gum, tobacco, } \\
\text { vanilla }\end{array}$ & 39,47 \\
\hline Don’t know & 2 & & 11,44 \\
\hline Don't know/other & 1 & & 27 \\
\hline Flavor & 1 & buttery, chocolate, cinnamon, menthol & 52 \\
\hline No flavor & 1 & & 52 \\
\hline Non-sweet & 1 & menthol, mint, tobacco & 3 \\
\hline Non-tobacco & 2 & cherry, peach, piña colada, vanilla & 38,43 \\
\hline Other & 7 & double espresso, pomegranate, vanilla bean & $5,11,36,39,44,46,49$ \\
\hline Other food & 1 & cupcakes, muffins & 34 \\
\hline Traditional flavors & 1 & menthol, tobacco & 50 \\
\hline
\end{tabular}

Main categories were clustered on similarity and marked with a color (alternating grey and white) to distinguish between similar categories. Individual e-liquid flavors in the third column are separated by a comma. If an e-liquid flavor was mentioned as example of a particular category in more than one article, prevalence is indicated. PG, propylene glycol; VG, vegetable glycerin.

\section{Similarities in flavor classifications across literature}

The category for alcohol-like flavors was named "alcohol" in each of the 6 articles using this category 11,27,34,36,42,44. Fruit-like flavors were classified as "fruit" in 18 articles 5,6,11-13,27,3437,40,42,44,45,47,49-51 ; only one of the articles reviewed used "cherry" as main category ${ }^{4}$. Articles commonly used a separate "spice" category 11,12,44,49; two articles used a "seasonings" category for flavors such as cinnamon and pepper ${ }^{35,45}$. Regarding beverages, five articles used a category for "beverages", "beverages/drinks", or "other beverages" 5,27,35,45,50. Furthermore, "candy" was a common category name for candy-like flavors ${ }^{27,33,34,42,44,46,49}$. Nineteen of the articles reviewed used a "tobacco" category for tobacco-like flavors ${ }^{4-6,11-13,27,34-45}$. Finally, 7 of the publications reviewed used an "unflavored" $12,27,40,48,51$ or "flavorless" category ${ }^{3,36}$, explained by Litt et al. ${ }^{40}$ and Rosbrook and Green ${ }^{48}$ as a propylene glycol/vegetable glycerin base only. In conclusion, common categories used in literature are "alcohol", "fruit", "spice", "beverages", "candy", "tobacco", and "unflavored".

\section{Differences in flavor classifications across literature}

The differences in the naming of main flavor categories in literature were mostly related to menthol-, nuts-, coffee-, dessert- and sweet-like flavors, and to unspecified categories. Whereas "menthol" was used as a separate category in 10 studies $4,11,35,399-42,44,45,48$, menthol was used in combination with "mint" $5,6,12,27,34,36,48$ or tobacco ${ }^{46,47}$ as well. Even though menthol and tobacco 
are clearly different, researchers might have clustered these flavors because of the definition of characterizing flavors in cigarettes (i.e., flavors other than tobacco or menthol) by the US FDA ${ }^{53}$, or by the fact that manufacturers commonly add menthol to tobacco products to increase sensory appeal ${ }^{9}$. Clustering menthol with mint flavor might be related to fact that menthol is the major constituent of oils that are produced by Mentha plants, which have the well-known cooling minty taste and smell ${ }^{54}$.

Regarding nut flavors, three studies used a separate "nut" category 5,35,45, while others combined it with "spices" ${ }^{27}$ or "candy" ${ }^{6}$. Similarly, 6 studies used a separate "coffee" category $4,6,11,36,37,44$, while coffee was classified together with "tea" ${ }^{27,34}$ or "alcohol" ${ }^{12,49}$ as well. "Dessert" was mentioned as a separate category in Table $2.1{ }^{51}$, or together with "bakery" ", "sweets" 27, "candy" 11,12, or "food/spice" 47. Dessert-like flavors were also classified as "cream" 35,45. Similarly, while "sweet" was a separate category in 7 studies $3,5,35,37,39,45,50$, some studies classified sweet flavors together with "dessert" ${ }^{27}$ or "chocolate" ${ }^{36}$. In addition, flavors such as vanilla and chocolate were used as main categories ${ }^{11,34,36,40}$, but were also part of the "sweet" category ${ }^{50}$. Finally, the final rows of Table 2.1 represent 10 unspecified flavor categories such as "flavor", "no flavor", "non-tobacco", "non-sweet", "other", "traditional flavors", and "don't know". Even though different names were used, the main categories described in this section could be summarized into "menthol", "nuts", "coffee", "dessert", "sweet", and "other flavors".

Besides differences in the naming of main categories, classification of specific e-liquid flavors within the main categories differed as well (third column of Table 2.1). Particularly e-liquids with a coffee, vanilla, and chocolate flavor were inconsistently classified: some articles classified these flavors within a different main category than others. Coffee-flavored e-liquids were classified within a separate category for "coffee/tea" ${ }^{27}$, or within a "beverages" 35,45,50 or "food/dessert/spice" category ${ }^{47}$. Vanilla-flavored e-liquids were classified within a broad range of categories, such as "candy or dessert" ${ }^{12}$, "candy" ${ }^{42}$, "food/dessert/spice" ${ }^{47}$, "cream" ${ }^{35,45}$, "sweet" ${ }^{50}$, and "dessert/sweets" ${ }^{27}$. Even though not consistently classified, vanilla seems a popular e-liquid flavor as it is mentioned as example of three of the unspecified categories for other flavors as well ${ }^{38,46,47}$. Similarly, besides being used as a separate category, chocolateflavored e-liquids were classified within 7 different flavor categories: " dessert", "candy or dessert", "candy", "cream", "sweet", "desert/sweets", and one of the unspecified categories $11,12,27,35,40,42,45,50-52$. Thus, vanilla and chocolate were not classified exclusively to one category such as "dessert", "candy" or "beverages". As vanilla and chocolate are often used as ingredients in sweet products, we consider these flavors general sweet flavors apart from candy, dessert or fruit.

\section{Proposed flavor wheel for e-liquids}

As a result of reviewing flavor classifications in literature, we propose a flavor wheel for e-liquids consisting of the following 13 main flavor categories: tobacco, menthol/mint, nuts, spices, coffeel tea, alcohol, other beverages, fruit, dessert, candy, other sweets, other flavors, and unflavored. Fruit flavors were divided into berries, citrus, tropical, and other fruits, similar to the division of the fruit category in the flavor wheels for wine, whiskey, coffee, and chocolate ${ }^{19,22,24,32}$. The e-liquid flavor wheel is shown in Figure 2.2. The subcategories in the outer layer of the flavor wheel are represented by the specific e-liquid flavors that were used in literature as examples 
of main categories (third column, Table 2.1). As the categories from our flavor wheel are fully based on flavor classifications from reviewed articles, they do not by definition represent each e-liquid flavor available. Therefore, our flavor wheel contains a category for other flavors in order to classify flavors that have not yet been mentioned in literature.

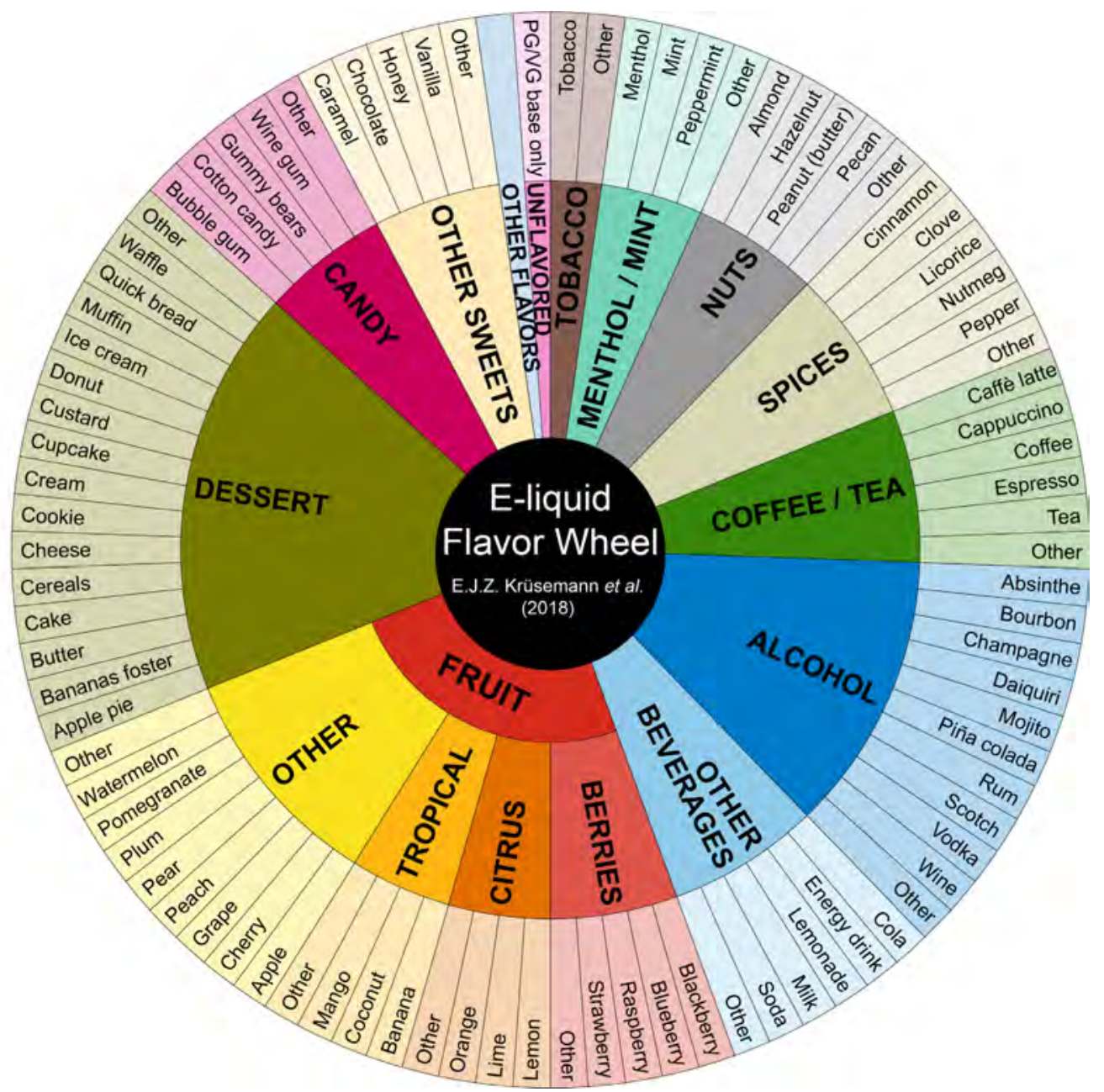

Figure 2.2: Proposed flavor wheel for classification of e-liquid flavors. The inner layer of the flavor wheel includes 13 main categories that were based on literature (first column, Table 2.1). The outer layer of the flavor wheel includes 90 subcategories that were extracted from the articles reviewed (third column, Table 2.1). 


\section{Discussion}

We reviewed literature to determine which e-liquid flavors and flavor categories have been used in research. There was large variation in the naming of main flavor categories, and e-liquid flavors were not consistently classified within these categories. To classify the excessive number of e-liquid flavors in a consistent way, we propose a flavor wheel for e-liquids (Figure 2.2). Our flavor wheel includes 13 main categories (inner wheel) and 90 subcategories (outer wheel). The categories from our flavor wheel are fully based on flavor classifications in literature, from different countries.

\section{Guideline for classifying e-liquid flavors using our proposed flavor wheel}

E-liquids are commonly classified based on marketing descriptions. Classifying e-cigarette flavors according to marketing involves brand names and flavor descriptions on packages or in advertisements. Flavor descriptions are used in promotion and marketing to create an association of the e-liquid's flavor with a particular product that the consumer knows and preferably likes. Using these marketing descriptions for flavor classification requires common rules, as brand names regularly change and flavor descriptions are sensitive to interpretation differences. For instance, this review showed that researchers classified a particular flavor in different categories (e.g., vanilla was classified as cream ${ }^{35,45}$, candy ${ }^{42}$, sweet ${ }^{50}$, dessert/candy ${ }^{12}$, or dessert/sweet ${ }^{27}$ ) Furthermore, e-liquids are not only marketed as single flavor such as strawberry or watermelon, but they can be associated with multiple flavor attributes. It could be questioned which of the flavor attributes should be used for classification, whether an e-liquid flavor can be associated with multiple categories, and how a distinction could be made between the "primary" flavor and "secondary" flavor attributes. For instance, of an e-liquid described as raspberry tea, is the primary flavor raspberry (e.g., fruit) or tea? Similarly, if an e-liquid has multiple flavor attributes such as "a hint of tobacco, banana, rum and custard", which of these attributes determines classification?

In order to minimize interpretation differences, to consistently classify e-liquids and distinguish primary from secondary flavors, we propose three steps as a guideline to classify e-liquid flavors using our flavor wheel:

Step 1: Distinguish primary from secondary e-liquid flavors. An e-liquid's primary flavor is based on the flavor description that is associated with a particular product as a whole. If the e-liquid does not describe a clear product as a whole, the primary flavor is the first flavor attribute mentioned. If present, other flavor attributes are considered secondary flavors.

Step 2: Classify an e-liquid's primary flavor in one of the 13 main categories as well as in one of the associated subcategories (inner wheel and outer wheel, respectively).

Step 3: Classify potential secondary flavors only in one of the subcategories (outer wheel).

The first step is based on the suggestion of Yingst et al. ${ }^{27}$ that flavors marketed as and meant to be associated with a particular product as a whole should be classified as a whole rather than the separate components of the e-liquid flavor. If an e-liquid's brand name or flavor description 
cannot be associated with a product as a whole but the description contains a list of equal flavor descriptors instead, the first flavor attribute mentioned is considered the primary flavor; other flavor descriptors are secondary flavors. Thus, using previous examples, for e-liquids flavored as "raspberry tea" or "watermelon combined with kiwi and lemon", we respectively consider tea and watermelon as primary flavors, whereas raspberry, kiwi, and lemon are secondary flavors. Furthermore, vanilla pudding and chocolate brownie would be classified as desserts, whereas e-liquids marketed purely as having vanilla or chocolate flavor would be classified as other sweets. Similarly, caramel candies such as toffee would be classified as candy, whereas e-liquids simply marketed as caramel would be classified as other sweets. Even though the flavor might be similar, we advise to use marketing descriptions of the product as a whole for classification in order to minimize interpretation differences.

Our proposal is based on the rationale that a secondary flavor, such as raspberry in raspberry tea, should be included as well, because it distinguishes raspberry tea from other types of tea and thus is an important specification of the product. Extra flavors attributes besides the primary flavor are considered secondary flavors. The second and third step suggest how to classify the primary and secondary flavors, respectively. The e-liquid flavor wheel contains 13 main categories (inner wheel) that are specified with 90 subcategories (outer wheel). According to the second step, the primary flavor should be classified in one of the main categories (inner wheel) and specified further in one of the associated subcategories (outer wheel), as the primary flavor is most important. According to the third step, secondary flavors, if present, should be classified only in one of the subcategories, as secondary flavors are solely meant for specification purposes. Examples of classifying primary as well as potential secondary flavors using marketing descriptions are provided in Table 2.2.

Following the three steps when applying our flavor wheel allows classifying e-liquids in a way that most closely represents the flavor as a whole. The advantage of our flavor wheel over a linear list of flavor categories is that no hierarchy of flavor categories exists, and the flavor wheel distinguishes main categories in the inner wheel from subcategories in the outer wheel, and thus primary from, if present, secondary flavor attributes. 
Table 2.2: Example of classifying e-liquids according to their primary and secondary flavors using the main and subcategories of our proposed flavor wheel shown in Figure 2.2. Classification is based on e-liquid marketing descriptions.

\begin{tabular}{|c|c|c|c|c|c|}
\hline E-liquid & Flavor description & $\begin{array}{l}\text { Main category } \\
\text { primary flavor } \\
\text { (inner wheel) }\end{array}$ & $\begin{array}{l}\text { Subcategory } \\
\text { primary flavor } \\
\text { (outer wheel) }\end{array}$ & $\begin{array}{l}\text { Secondary } \\
\text { flavor? (yes/ } \\
\text { no) }\end{array}$ & $\begin{array}{l}\text { Subcategories } \\
\text { secondary flavor } \\
\text { (outer wheel) }\end{array}$ \\
\hline 1 & Raspberry tea & Coffee/tea & Tea & Yes & Raspberry \\
\hline 2 & $\begin{array}{l}\text { Watermelon } \\
\text { combined with kiwi } \\
\text { and lemon }\end{array}$ & Fruit & Watermelon & Yes & Kiwi, lemon \\
\hline 3 & $\begin{array}{l}\text { Strawberry with a } \\
\text { hint of menthol }\end{array}$ & Fruit & Strawberry & Yes & Menthol \\
\hline 3 & Chocolate & Other sweets & Chocolate & No & - \\
\hline $\begin{array}{l}4 \\
\text { etc. }\end{array}$ & Bubble gum & Candy & Bubble gum & No & - \\
\hline
\end{tabular}

\section{Applications in research}

Our flavor wheel could be applied in multiple research disciplines. For instance, it could be used as a guideline in experimental study designs to select a representative sample of e-liquid flavors from different categories. In addition, e-liquid sales numbers could reveal information on popularity of particular flavors or flavor categories, and how demand of these e-liquid flavors persists over time. Using chemical-analytical research, flavor compositions of e-liquids could be compared. A large number of e-liquids could be measured using gas chromatography - mass spectrometry to investigate which flavor molecules are frequently present in e-liquids with particular flavors, and might thus be responsible for a particular flavor or flavor category from our flavor wheel. In sensory research on e-liquid flavors, the categories from the flavor wheel could be used as flavor attributes. E-liquids could be assessed by a panel of consumers or trained experts based on the intensity of particular flavor attributes to create a flavor profile. Flavor profiles created by panelists could be compared to e-liquid marketing descriptions to investigate to what extent consumers identify primary and potential secondary flavor attributes.

In addition, our flavor wheel can be used in research investigating liking and disliking of particular e-liquid flavors or flavor categories among different consumer groups such as smoking adults and non-smoking youth. For instance, results of the studies included in this review on flavor liking show that e-cigarette users in general mostly prefer and/or use tobacco-flavored e-liquids and e-liquids with a sweet or fruit flavor 3,5,6,12,27,34-36,38,44,45,47. Males mostly seem to prefer tobacco-flavored e-liquids, whereas non-tobacco (particularly sweet) flavors are more popular among females ${ }^{36,43}$. Comparing adults with adolescents, sweet flavors are particularly popular among young e-cigarette users, while non-sweet flavors such as tobacco are more common among adults ${ }^{11,12,39}$. Comparing smokers with non-smokers, (adult) smokers are more interested in trying e-cigarettes with a tobacco or menthol flavor ${ }^{4,39,40,44,46}$, whereas (younger) non-smokers are more interested in trying fruit and sweet flavors ${ }^{4,37,42}$. These conclusions show 
that sweet e-liquids are interesting for research on flavor liking. However, our review showed that "sweet"-flavored e-liquids have been classified inconsistently across literature (Table 2.1), which may cause difficulties in data interpretation. Our flavor wheel provides a guideline to distinguish e-liquids with a dessert, candy and beverage flavor from other sweet flavors such as vanilla or chocolate. Applying our flavor wheel in research on flavor liking will thus help to minimize interpretation differences and increase comparability of research results. Furthermore, our flavor wheel can be used to specify liking of main flavor categories into liking of specific e-liquid flavors (outer wheel) among different consumer groups.

Flavor liking in e-liquids could also be compared to liking and disliking of food products, as vaping and eating can both be considered forms of ingestive behavior (i.e., the same route of administration [via nose and mouth] is followed, and the same type of psychological processes of perception and reward may be triggered). Flavors are important in both vaping and eating. For instance, children and adolescents have a high preference for sweet tastes and odors ${ }^{55}$, which might explain why particularly sweet, dessert, and candy flavored e-cigarettes are popular among youth ${ }^{3,4,12,17,37,42}$. It would be interesting to further investigate similarities and differences between vaping and eating in relation to perception and reward.

In addition, our flavor wheel could be compared to flavor classifications in the food, alcohol, and fragrance industries, for instance to investigate whether availability of e-liquid flavors is related to flavors that are commonly used in other products. A preliminary comparison between our e-liquid flavor wheel and the coffee, chocolate, wine, beer, whiskey, cigar, and fragrance wheels shows similarities and differences. For instance, each of the flavor wheels has a fruit category in their inner wheel ${ }^{19,20,22,24-26,32}$. Similar to our wheel, categories for respectively nuts and spices are present in the inner wheels of the coffee, cigar, wine, and chocolate flavor wheel 22,24,26,32. Whereas tobacco is a main category in our flavor wheel, it is a subcategory of the brown fruit category of the chocolate wheel ${ }^{22}$, the dried vegetative category of the wine aroma wheel ${ }^{32}$, the plants category of the cigar flavor wheel ${ }^{26}$, and the roasted category of the coffee flavor wheel ${ }^{24}$. The candy, other beverages, and dessert categories of our e-liquid wheel represent products as a whole, which are not recognized in other flavor wheels except for the chocolate wheel, which includes subcategories such as cheesecake, butterscotch, toffee, candy "fruit tarts", and a type of chocolate cake ${ }^{22}$. The menthol/mint is a main category in our e-cigarette flavor wheel, whereas only the wine aroma wheel has a menthol subcategory ${ }^{32}$. The main difference between the e-liquid and food flavor wheels is that our flavor wheel does not contain a floral category, while each of the other flavor wheels investigated has a floral category in their inner wheel 19,20,22,24-26,32. Strikingly, even though one article used "cheese" as part of their "cream" category ${ }^{35}$, none of the articles reviewed used a main category for savory flavors, while research shows similar liking and reward for both sweet and savory food products ${ }^{56}$. Because our flavor wheel is based on e-liquid flavors that have been used in research, it does not mean that no floral or savory flavored e-liquids exist. It would be interesting to investigate how many e-liquids with a floral or savory flavor are available, and how liking of these e-liquids relates to liking of savory and floral-flavored food products.

Our flavor wheel might also be used for development and analysis of survey items. For instance, researchers could use the main and/or subcategories of the flavor wheel as answer options for multiple choice questions related to e-liquid flavor use and/or preferences. The flavor 
categories could also be used to (manually) classify open-ended responses from consumers to similar survey questions. In this way, the flavor wheel facilitates communication between researchers and real-world users, which helps to understand consumer liking and disliking of certain e-liquid flavors.

\section{Applications in policy}

Consistent classification of e-liquid flavors by consumers as well as researchers will improve data accuracy, minimize interpretation differences, and increase comparability of research results across studies. Research results could be used by policy makers for regulation of particular e-liquid flavors or flavor categories from the flavor wheel. The classification rules from Yingst et al. ${ }^{27}$ were based on the possibility that the same regulations for flavors in cigarettes would be applied to e-liquids. In cigarettes, characterizing flavors have been prohibited, which are defined by the European Union as "flavors other than the one of tobacco" and by the United States Food and Drug Administration as "flavors other than tobacco or menthol" 53,57. Therefore, Yingst et al. ${ }^{27}$ aimed to distinguish e-liquids with an exclusive tobacco flavor from e-liquids also having other flavor attributes. According to their classification rules, e-liquids marketed as "pipe tobacco with a hint of cherry" would be classified as fruit. However, as all e-liquids have a flavor, it might be difficult to compare e-liquids with cigarettes from a regulation point of view. Furthermore, considering the product as a whole and the first flavor mentioned, the primary flavor attribute of the example according to our flavor wheel would be tobacco. According to our proposal, this e-liquid would be classified in the main tobacco category (inner wheel) with pipe tobacco as subcategory (outer wheel), with an additional secondary flavor in a cherry subcategory (outer wheel). Our flavor wheel thus allows to distinguish e-liquids with a primary tobacco flavor from e-liquids marketed as having a primary tobacco flavor and additional secondary flavors other than tobacco. In this way, each of the flavor attributes that are used for marketing of e-liquids could be considered for regulation of e-liquid flavors.

Furthermore, (characterizing) flavors in tobacco cigarettes are prohibited because they increase attractiveness and thereby facilitate smoking initiation among young people ${ }^{58,59}$. Flavors in e-cigarettes are not only attractive to young people, but are also associated with higher rates of smoking cessation among adults ${ }^{6}$. Sensory research using our flavor wheel will provide more insight in liking of e-liquid flavors and/or flavor categories among these different consumer groups. Policy makers could use research results to regulate e-liquid flavors in a way that e-cigarettes are attractive to adult smokers and unattractive to young non-smokers.

\section{Future research}

The categories from our flavor wheel should be corroborated to determine whether the wheel is complete or additional categories are required. For instance, as categories from our flavor wheel were mainly based on studies performed in the United States, research on e-liquid flavors offered by retail Web sites from different countries might identify new or other flavors that are not covered by our flavor wheel. Preliminary market observations have revealed the availability of e-liquid flavors that have not been used in the study design of the articles reviewed, such as rose and chicken. E-liquids flavored as such would be classified in the other flavor category of our flavor wheel. Similar to the need for modification of the wine aroma wheel ${ }^{32}$, future research 
might reveal the need to specify the category for other flavors into additional categories such as "floral" or "savory". Future research should also investigate if our flavor wheel is complete and not open to misinterpretation by having a panel of consumers classify a large sample of e-liquid flavor descriptions on the basis of the proposed flavor wheel. Statistical data on e-liquid classification by the panel will show if panelists follow the classification steps and apply the flavor wheel in a consistent, repeatable, and reproducible way.

\section{Conclusions}

A large variation in the naming of flavor categories was found in literature, and e-liquid flavors were not consistently classified. We propose an e-liquid flavor wheel including three steps for systematic classification of e-liquids based on their marketing descriptions. The flavor wheel includes 13 main categories (inner wheel) and 90 subcategories (outer wheel) that aim to create a shared flavor vocabulary for a broad range of potential users. Applying the flavor wheel in research will minimize interpretation differences, increase comparability of research results, and support policy makers in developing rules for regulation of e-liquid flavors. 


\section{Appendix}

Table A2.1: PubMed database search strategy.

\begin{tabular}{lll}
\hline Search & Query & Items found \\
\hline$\# 19$ & Search (\#8 or \#11 or \#13 or \#14 or \#16 or \#17) & 197 \\
& Filters: Publication date from 1990/01/01 to 2017/05/17 & \\
$\# 18$ & Search (\#8 or \#11 or \#13 or \#14 or \#16 or \#17) & 214 \\
$\# 17$ & Search ((\#4 or \#9) and \#15) & 4 \\
$\# 16$ & Search ((\#5 or \#10) and \#15) & 140 \\
$\# 15$ & Search (learning*[Title] or wanting*[Title] or liking*[Title]) & 74125 \\
$\# 14$ & Search (\#9 and \#12) & 13 \\
$\# 13$ & Search (\#4 and \#12) & 14 \\
$\# 12$ & Search “perception"[MeSH Major Topic] & 214642 \\
$\# 11$ & Search (\#9 and \#10) & 21 \\
$\# 10$ & Search “flavoring agents"[MeSH Major Topic] & 7989 \\
$\# 9$ & Search electronic cigarette[MeSH Major Topic] & 1014 \\
$\# 8$ & Search (\#4 and \#7) & 47 \\
$\# 7$ & Search (\#5 or \#6) & 4049 \\
$\# 6$ & Search (consumer*[Title] and preference*[Title]) & 261 \\
$\# 5$ & Search (flavour*[Title] or flavor*[Title]) & 3797 \\
$\# 4$ & Search (\#1 or \#2 or \#3) & 1855 \\
$\# 3$ & Search electronic[Title] and nicotine[Title] & 205 \\
$\# 2$ & Search electronic cigar*[Title] & 900 \\
$\# 1$ & Search e-cigar*[Title] & 889 \\
\hline
\end{tabular}


Table A2.2: Characteristics of the included publications $(n=28)$.

\begin{tabular}{|c|c|c|c|}
\hline $\begin{array}{l}\text { Authors, year } \\
\text { (country) }\end{array}$ & $\begin{array}{l}\text { Study population, } \\
\text { sample size and mean } \\
\text { age (SD) }\end{array}$ & Study design & Flavor classification \\
\hline $\begin{array}{l}\text { Audrain- } \\
\text { McGovern et } \\
\text { al, } 2016 \text { (USA) } \\
51\end{array}$ & $\begin{array}{l}\text { Young adult smokers, } \mathrm{n}= \\
32, \text { mean age } 25.0(3.0)\end{array}$ & $\begin{array}{l}\text { Experimental } \\
\text { laboratory } \\
\text { session }\end{array}$ & $\begin{array}{l}\text { Unflavored } \\
\text { Fruit (green apple) } \\
\text { Dessert (chocolate) }\end{array}$ \\
\hline $\begin{array}{l}\text { Berg, } 2016 \\
\text { (USA) }^{34}\end{array}$ & $\begin{array}{l}\text { Adults aged } 18-34 \text { years } \\
\text { living in US, } \mathrm{n}=1567 \text {, } \\
\text { mean age } 25.2(5.1)\end{array}$ & $\begin{array}{l}\text { Cross- } \\
\text { sectional } \\
\text { survey }\end{array}$ & $\begin{array}{l}\text { Fruit } \\
\text { Caramel, vanilla, chocolate or cream } \\
\text { Candy (e.g., licorice, gummy bears) } \\
\text { Menthol/mint } \\
\text { Tobacco } \\
\text { Coffee/tea } \\
\text { Alcohol (e.g., mojitos, daiquiris) } \\
\text { Other food (e.g., cupcakes, muffins) }\end{array}$ \\
\hline $\begin{array}{l}\text { Chen and Zeng, } \\
2017 \text { (USA)*35 }\end{array}$ & $\begin{array}{l}\text { 14,433 e-liquid reviews } \\
\text { from the JuiceDB website } \\
\text { between June } 2013 \text { and } \\
\text { November } 2015\end{array}$ & $\begin{array}{l}\text { Longitudinal } \\
\text { content } \\
\text { analysis }\end{array}$ & $\begin{array}{l}\text { Fruit (strawberry, banana, apple, } \\
\text { blueberry, mango, cherry, orange, lemon, } \\
\text { waterlemon, raspberry, pomegranate, pear } \\
\text { plum, grape, lime) } \\
\text { Cream (cream, vanilla, custard, milk, } \\
\text { chocolate, cake, cookie, cheese, butter) } \\
\text { Tobacco (tobacco) } \\
\text { Menthol (menthol, mint) } \\
\text { Beverages (coffee, tea, wine) } \\
\text { Sweet (candy, honey, caramel) } \\
\text { Seasonings (cinnamon, pepper) } \\
\text { Nuts (nuts) }\end{array}$ \\
\hline $\begin{array}{l}\text { Chu et al, } 2015 \\
\text { (USA) }{ }^{52}\end{array}$ & $\begin{array}{l}6 \text { months of tweets from } \\
2 \text { e-cigarette brands (Blu } \\
\text { owned by Lorillard and } \\
\text { V2 owned by VMR), } n= \\
1180\end{array}$ & $\begin{array}{l}\text { Longitudinal } \\
\text { content } \\
\text { analysis }\end{array}$ & $\begin{array}{l}\text { Flavor (e.g., buttery, menthol, chocolate, } \\
\text { cinnamon, and so on - excluding tobacco) } \\
\text { No flavor }\end{array}$ \\
\hline $\begin{array}{l}\text { Cooper } \text { et al, } \\
2016 \text { (USA) }{ }^{49}\end{array}$ & $\begin{array}{l}\text { A probability-design } \\
\text { sample of public schools. } \\
\text { Data from a rapid } \\
\text { response surveillance } \\
\text { system (TATAMS) with } \\
\text { 6th, 8th and 10th grade } \\
\text { students ( } \mathrm{N}=434,601, \mathrm{n} \\
=3704)\end{array}$ & $\begin{array}{l}\text { Cross- } \\
\text { sectional } \\
\text { survey }\end{array}$ & $\begin{array}{l}\text { Mint } \\
\text { Candy } \\
\text { Fruit } \\
\text { Coffee/alcohol } \\
\text { Spice } \\
\text { Other }\end{array}$ \\
\hline $\begin{array}{l}\text { Czoli et al, } \\
2016 \text { (Canada) }^{4}\end{array}$ & $\begin{array}{l}\text { Non-smoking youth and } \\
\text { young adults aged } 16-24 \\
\text { years, mean age } 20.6 \\
(2.8) \text {; smoking youth and } \\
\text { young adults aged } 16-24 \\
\text { years, mean age } 21.4 \\
(2.1) \text {; and smoking adults } \\
\text { aged } 25 \text { years and older, } \\
\text { mean age } 49.0 \text { (12.2). } \\
\text { Recruited through GMI, n } \\
=915 \text {. }\end{array}$ & $\begin{array}{l}\text { Experimental } \\
\text { online } \\
\text { assessment }\end{array}$ & $\begin{array}{l}\text { Tobacco } \\
\text { Menthol } \\
\text { Coffee } \\
\text { Cherry }\end{array}$ \\
\hline
\end{tabular}




\begin{tabular}{|c|c|c|c|}
\hline $\begin{array}{l}\text { Dawkins et al, } \\
2013 \text { (UK) }^{36}\end{array}$ & $\begin{array}{l}\text { A restricted sample of } \\
\text { TECC and Totally Wicked } \\
\text { E-Liquid (TWEL) users } \\
\text { (the two most widely- } \\
\text { used brands in the } \\
\text { UK) recruited via their } \\
\text { websites, } n=1347 \text {, mean } \\
\text { age } 43.39(11.99)\end{array}$ & $\begin{array}{l}\text { Cross- } \\
\text { sectional } \\
\text { survey }\end{array}$ & $\begin{array}{l}\text { Tobacco } \\
\text { Fruit } \\
\text { Menthol/mint } \\
\text { Chocolate/sweet } \\
\text { Coffee } \\
\text { Other } \\
\text { Vanilla } \\
\text { Alcohol } \\
\text { Flavorless }\end{array}$ \\
\hline $\begin{array}{l}\text { Farsalinos et al, } \\
2013 \text { (Greece / } \\
\text { Italy) }{ }^{5}\end{array}$ & $\begin{array}{l}\text { Dedicated adult } \\
\text { e-cigarette users of any } \\
\text { age, } n=4618, \text { median age } \\
40(32-49 \text { interquartile } \\
\text { range). }\end{array}$ & $\begin{array}{l}\text { Cross- } \\
\text { sectional } \\
\text { survey }\end{array}$ & $\begin{array}{l}\text { Tobacco } \\
\text { Menthol/mint } \\
\text { Sweet } \\
\text { Nuts } \\
\text { Fruit } \\
\text { Beverages/drinks } \\
\text { Other }\end{array}$ \\
\hline $\begin{array}{l}\text { Ford et al, } 2016 \\
\left(\text { UK) }{ }^{37}\right.\end{array}$ & $\begin{array}{l}\text { Data from the } 2014 \text { Youth } \\
\text { Tobacco Policy Survey } \\
\text { among } 11-16 \text { year olds } \\
\text { across the UK, } n=1205 \text {, } \\
\text { mean age } 13.5\end{array}$ & $\begin{array}{l}\text { Cross- } \\
\text { sectional } \\
\text { survey }\end{array}$ & $\begin{array}{l}\text { Tobacco } \\
\text { Fruit (cherry) } \\
\text { Sweet (candy floss) } \\
\text { Coffee }\end{array}$ \\
\hline $\begin{array}{l}\text { Goldenson et } \\
a l, 2016 \text { (USA) } \\
3\end{array}$ & $\begin{array}{l}\text { Young adult vapers aged } \\
19-34 \text { years, } n=20 \text {, mean } \\
\text { age } 26.3 \pm 4.6\end{array}$ & $\begin{array}{l}\text { Experimental } \\
\text { laboratory } \\
\text { session }\end{array}$ & $\begin{array}{l}\text { Sweet (peach,watermelon, blackberry, } \\
\text { cotton candy, cola and sweet lemon tea) } \\
\text { Non-sweet (mint, tobacco and menthol) } \\
\text { Flavorless }\end{array}$ \\
\hline $\begin{array}{l}\text { Harrell et al, } \\
2017 \text { (USA) }{ }^{12}\end{array}$ & $\begin{array}{l}\text { (A) Youth aged } 12-17 \\
\text { years recruited from } \\
\text { TATAMS }(\mathrm{N}=461,069, \mathrm{n} \\
=3907) \text {; } \\
\text { (B) Young adults aged } 18- \\
29 \text { years recruited from } \\
\text { M-PACT }(\mathrm{N}=13,714, \mathrm{n}= \\
5482) ; \\
\text { (C) Older adults aged } \\
30+\text { years recruited from } \\
\text { TPRPS }(\mathrm{N}=8135, \mathrm{n}= \\
6015)\end{array}$ & $\begin{array}{l}\text { Cross- } \\
\text { sectional } \\
\text { survey }\end{array}$ & $\begin{array}{l}\text { Tobacco } \\
\text { Menthol/mint } \\
\text { Fruit (e.g., cherry, strawberry) } \\
\text { Candy or dessert (e.g., respectively gummy } \\
\text { bear or chocolate/vanilla) } \\
\text { Coffee/alcohol } \\
\text { Spice (e.g., cinnamon) } \\
\text { Unflavored }\end{array}$ \\
\hline $\begin{array}{l}\text { Kim et al, } 2016 \\
\text { (USA) }^{38}\end{array}$ & $\begin{array}{l}\text { E-cigarette sole or dual } \\
\text { users, } n=31, \text { mean age } \\
33.6 \pm 10.9\end{array}$ & $\begin{array}{l}\text { Experimental } \\
\text { laboratory } \\
\text { session }\end{array}$ & $\begin{array}{l}\text { Tobacco (tobacco and menthol: resp. } \\
\text { Classic Tobacco and Magnificent Menthol) } \\
\text { Non-tobacco (cherry, piña colada, peach, } \\
\text { vanilla: resp. Cherry Crush, Piña Colada, } \\
\text { Peach Schnapps, and Vivid Vanilla) }\end{array}$ \\
\hline $\begin{array}{l}\text { Krishnan-Sarin } \\
\text { et al, } 2015 \\
\text { (USA) }^{39}\end{array}$ & $\begin{array}{l}\text { (A) High school students } \\
\text { from Connecticut, } \mathrm{n}= \\
3614, \text { mean age } 15.63 \\
(1.20) \\
\text { (B) Middle school } \\
\text { students from } \\
\text { Connecticut, } \mathrm{n}=1166 \text {, } \\
\text { mean age } 12.18(0.90)\end{array}$ & $\begin{array}{l}\text { Cross- } \\
\text { sectional } \\
\text { survey }\end{array}$ & $\begin{array}{l}\text { Menthol } \\
\text { Tobacco } \\
\text { Sweet } \\
\text { Combination of flavors } \\
\text { Other }\end{array}$ \\
\hline $\begin{array}{l}\text { Litt et al, } 2016 \\
\text { (USA) }\end{array}$ & $\begin{array}{l}\text { Current cigarette smokers } \\
\text { aged } 18-55 \text { years, } \mathrm{n}=88, \\
\text { mean age } 36.3(10.3)\end{array}$ & $\begin{array}{l}\text { Experimental } \\
\text { laboratory } \\
\text { session and } \\
\text { field study }\end{array}$ & $\begin{array}{l}\text { Unflavored ( } P G / V G \text { base only) } \\
\text { Tobacco } \\
\text { Menthol } \\
\text { Fruit (cherry) } \\
\text { Chocolate (chocolate) }\end{array}$ \\
\hline
\end{tabular}




\begin{tabular}{|c|c|c|c|}
\hline $\begin{array}{l}\text { Morean et al, } \\
2018 \text { (USA)*11 }\end{array}$ & $\begin{array}{l}\text { Adolescent past-month } \\
\text { e-cigarette users from } 5 \\
\text { high schools, } \mathrm{n}=396, \\
\text { mean age } 16.18(1.18) \text {; } \\
\text { adult past month-e- } \\
\text { cigarette users, } \mathrm{n}=590 \text {, } \\
\text { mean age } 34.25(9.89)\end{array}$ & $\begin{array}{l}\text { Cross- } \\
\text { sectional } \\
\text { survey }\end{array}$ & $\begin{array}{l}\text { Tobacco } \\
\text { Menthol } \\
\text { Mint } \\
\text { Fruit (e.g., strawberry, blueberry, or peach) } \\
\text { Vanilla } \\
\text { Candy/dessert (e.g., apple pie, chocolate, } \\
\text { or Jolly Rancher) } \\
\text { Spice (e.g., clove, cinnamon or nutmeg) } \\
\text { Alcohol (e.g., piña colada, strawberry } \\
\text { daiquiri, or bourbon) } \\
\text { Coffee (e.g., espresso, latte, or cappuccino) } \\
\text { Other } \\
\text { Don't know }\end{array}$ \\
\hline $\begin{array}{l}\text { Oncken et al, } \\
2015 \text { (USA) }{ }^{41}\end{array}$ & $\begin{array}{l}\text { Non-treatment seeking } \\
\text { smokers who were willing } \\
\text { to try e-cigarettes for } 2 \\
\text { weeks and abstain from } \\
\text { cigarette smoking, } \mathrm{n}=20 \text {, } \\
\text { mean age } 42.2(9.7)\end{array}$ & $\begin{array}{l}\text { Experimental } \\
\text { field study } \\
\text { and laboratory } \\
\text { session, and } \\
\text { cross-sectional } \\
\text { survey }\end{array}$ & $\begin{array}{l}\text { Menthol (menthol tobacco) } \\
\text { Tobacco }\end{array}$ \\
\hline $\begin{array}{l}\text { Pepper } \text { et al, } \\
2016 \text { (USA) }{ }^{42}\end{array}$ & $\begin{array}{l}\text { A national probability } \\
\text { sample of USA } \\
\text { adolescents aged } 13-17 \\
\text { years, } \mathrm{n}=1125, \text { mean age } \\
15.1(1.4)\end{array}$ & $\begin{array}{l}\text { Experimental } \\
\text { survey }\end{array}$ & $\begin{array}{l}\text { Tobacco } \\
\text { Alcohol (e.g., scotch or champagne) } \\
\text { Menthol } \\
\text { Candy (e.g., chocolate or vanilla) } \\
\text { Fruit (e.g., cherry or peach) }\end{array}$ \\
\hline $\begin{array}{l}\text { Piñeiro et al, } \\
2015 \text { (USA) }{ }^{43}\end{array}$ & $\begin{array}{l}\text { E-cig users aged } 18-29 \\
30-44,45-59, \text { and } \geq 60 \\
\text { years, } \mathrm{n}=1815\end{array}$ & $\begin{array}{l}\text { Cross- } \\
\text { sectional } \\
\text { survey }\end{array}$ & $\begin{array}{l}\text { Tobacco } \\
\text { Non-tobacco }\end{array}$ \\
\hline $\begin{array}{l}\text { Rosbrook and } \\
\text { Green, } 2016 \\
\text { (USA) } 48\end{array}$ & $\begin{array}{l}\text { Adult daily smokers aged } \\
18-45 \text { years, } \mathrm{n}=32\end{array}$ & $\begin{array}{l}\text { Experimental } \\
\text { laboratory } \\
\text { session }\end{array}$ & $\begin{array}{l}\text { Menthol } \\
\text { Menthol/mint } \\
\text { Unflavored (PG/VG base only) }\end{array}$ \\
\hline $\begin{array}{l}\text { Shiffman et al, } \\
2015 \text { (USA) }{ }^{46}\end{array}$ & $\begin{array}{l}\text { Non-smoking teens aged } \\
13-17 \text { years, mean age } \\
15.9(1.1) ; \text { adult smokers } \\
\text { aged } 19-80 \text { years, mean } \\
\text { age } 43.7(14.5) ; \mathrm{n}=648\end{array}$ & $\begin{array}{l}\text { Experimental } \\
\text { online } \\
\text { assessment }\end{array}$ & $\begin{array}{l}\text { Tobacco or menthol (tobacco, menthol: } \\
\text { Classic Tobacco, Menthol, Dark Tobacco } \\
\text { Blend) } \\
\text { Candy (bubble gum and gummy bear: } \\
\text { Bubble Gum, Cotton Candy, Gummy Bear) } \\
\text { Other (e.g., pomegrenate, vanilla bean, } \\
\text { double espresso: Black \& Blue Berry, } \\
\text { Blood Orange, Butter Crunch, Double } \\
\text { Espresso, Peach Tea, Pomegrenate, } \\
\text { Raspberry, Single Malt Scotch, Vanilla } \\
\text { Bean) }\end{array}$ \\
\hline $\begin{array}{l}\text { Shiplo et al, } \\
2015 \text { (Canada) } \\
44\end{array}$ & $\begin{array}{l}\text { Younger non-smokers } \\
\text { (mean age 20.51), younger } \\
\text { smokers (21.35), and } \\
\text { older smokers (48.52). } \\
\text { Recruited through GMI, n } \\
=1095\end{array}$ & $\begin{array}{l}\text { Cross- } \\
\text { sectional } \\
\text { survey }\end{array}$ & $\begin{array}{l}\text { Fruit } \\
\text { Menthol } \\
\text { Tobacco } \\
\text { Candy } \\
\text { Coffee } \\
\text { Spice } \\
\text { Alcohol } \\
\text { Other } \\
\text { Don’t know }\end{array}$ \\
\hline
\end{tabular}




\begin{tabular}{|c|c|c|c|}
\hline $\begin{array}{l}\text { Soule et al, } \\
2016 \text { (USA) }{ }^{47}\end{array}$ & $\begin{array}{l}\text { Adult experienced e-cig } \\
\text { users, } \mathrm{n}=46 \text {, mean age } \\
38.5(10.52)\end{array}$ & $\begin{array}{l}\text { Mixed- } \\
\text { Method: } \\
\text { online } \\
\text { assessment } \\
\text { and survey }\end{array}$ & $\begin{array}{l}\text { Food/Dessert/Spice (e.g., vanilla, banana } \\
\text { foster, peaches, coffee) } \\
\text { Fruit (e.g., watermelon, mango) } \\
\text { Tobacco or menthol } \\
\text { Combination of flavors (e.g., bubble gum, } \\
\text { blueberry champagne, vanilla and tobacco) }\end{array}$ \\
\hline $\begin{array}{l}\text { St.Helen et al, } \\
2017 \text { (USA)*13 }\end{array}$ & $\begin{array}{l}\text { Exclusive e-cigarette users } \\
\text { or dual users }(<5 \text { cig/ } \\
\text { day }), \mathrm{n}=14 \text {, mean age } \\
32.3(13.8)\end{array}$ & $\begin{array}{l}\text { Experimental } \\
\text { laboratory } \\
\text { session }\end{array}$ & $\begin{array}{l}\text { Fruit (strawberry) } \\
\text { Tobacco (tobacco) }\end{array}$ \\
\hline $\begin{array}{l}\text { Tackett et al, } \\
2015\left(\text { USA) }{ }^{6}\right.\end{array}$ & $\begin{array}{l}\text { Adult vape store } \\
\text { customers at four } \\
\text { retail locations in the } \\
\text { Midwestern United States, } \\
\mathrm{n}=215 \text {, mean age } 36.2 \\
(13.0)\end{array}$ & $\begin{array}{l}\text { Cross- } \\
\text { sectional } \\
\text { survey }\end{array}$ & $\begin{array}{l}\text { Fruit (e.g., strawberry, blueberry) } \\
\text { Bakery/dessert } \\
\text { Tobacco } \\
\text { Menthol/mint } \\
\text { Candy/nuts (e.g., cotton candy, SweetTart, } \\
\text { Hazelnut, Almond) } \\
\text { Coffee }\end{array}$ \\
\hline $\begin{array}{l}\text { Vasiljevic et al, } \\
2015 \text { (UK) }{ }^{33}\end{array}$ & $\begin{array}{l}\text { English school children } \\
\text { aged } 11-16 \text { years, } \mathrm{n}= \\
598, \text { mean age } 13.16 \\
(1.46)\end{array}$ & $\begin{array}{l}\text { Experimental } \\
\text { exposure } \\
\text { assessment }\end{array}$ & Candy \\
\hline $\begin{array}{l}\text { Wang et al, } \\
2015 \text { (China / } \\
\text { USA) }{ }^{45}\end{array}$ & $\begin{array}{l}27,638 \text { flavor-related posts } \\
\text { and } 7,376 \text { brand-related } \\
\text { posts in } 10 \text { subreddit } \\
\text { communities }\end{array}$ & $\begin{array}{l}\text { Longitudinal } \\
\text { content } \\
\text { analysis }\end{array}$ & $\begin{array}{l}\text { Fruit (strawberry, banana, apple, peach, } \\
\text { blueberry, mango, cherry, orange, lemon, } \\
\text { watermelon, raspberry, pomegranate) } \\
\text { Cream (vanilla, custard, milk, chocolate, } \\
\text { cake, cookie) } \\
\text { Tobacco } \\
\text { Menthol (menthol, mint) } \\
\text { Beverages (coffee, tea, wine) } \\
\text { Sweet (candy, honey) } \\
\text { Seasonings (cinnamon, pepper) } \\
\text { Nuts (nuts) }\end{array}$ \\
\hline $\begin{array}{l}\text { Yingst et al, } \\
2015 \text { (USA) }\end{array}$ & $\begin{array}{l}\text { E-cig users, } n=4421 \text {, } \\
\text { mean age } 40.1(12.7)\end{array}$ & $\begin{array}{l}\text { Cross- } \\
\text { sectional } \\
\text { survey }\end{array}$ & $\begin{array}{l}\text { Traditional flavors (tobacco or menthol) } \\
\text { Fruit (e.g., cherry, berry, apple) } \\
\text { Sweet (e.g., chocolate, vanilla, desserts, } \\
\text { candies) } \\
\text { Beverages (e.g., coffee, alcoholic drinks, } \\
\text { soda) }\end{array}$ \\
\hline $\begin{array}{l}\text { Yingst et al, } \\
2017 \text { (USA) }{ }^{27}\end{array}$ & $\begin{array}{l}\text { Current e-cig users, who } \\
\text { were either current or } \\
\text { former smokers, at least } \\
18 \text { years of age, } n=3716 \text {, } \\
\text { mean age } 40.4\end{array}$ & $\begin{array}{l}\text { Cross- } \\
\text { sectional } \\
\text { survey }\end{array}$ & $\begin{array}{l}\text { Tobacco } \\
\text { Menthol/mint (menthol, mint, peppermint) } \\
\text { Fruit (e.g., apple, strawberry, coconut, } \\
\text { orange, berries) } \\
\text { Dessert/sweets (e.g., chocolate, vanilla, } \\
\text { quick breads, cakes, waffles, donuts, } \\
\text { cereals, and ice cream) } \\
\text { Alcohol (e.g., rum, absinthe, absolut) } \\
\text { Nuts/spices (e.g., peanut butter, almond, } \\
\text { cinnamon, pecan) } \\
\text { Candy (e.g., licorice, sweetTARTS, gummy } \\
\text { bears, Swedish fish) } \\
\text { Coffee/tea (e.g., coffee, tea, espresso, } \\
\text { cappuccino) } \\
\text { Other beverages (e.g., sodas, energy drinks, } \\
\text { lemonades) } \\
\text { Unflavored } \\
\text { Don't know/other }\end{array}$ \\
\hline
\end{tabular}

*Articles included as result of the literature search update $(n=3)$.

PG, propylene glycol; VG, vegetable glycerin. 


\section{References}

1. Bertholon JF, Becquemin MH, Annesi-Maesano I, Dautzenberg B. Electronic cigarettes: a short review. Respiration. 2013;86(5):433-438.

2. Zhu SH, Sun JY, Bonnevie E, et al. Four hundred and sixty brands of e-cigarettes and counting: implications for product regulation. Tob Control. 2014;23 Suppl 3:iii3-9.

3. Goldenson NI, Kirkpatrick MG, Barrington-Trimis JL, et al. Effects of sweet flavorings and nicotine on the appeal and sensory properties of e-cigarettes among young adult vapers: Application of a novel methodology. Drug Alcohol Depen. 2016;168:176-180.

4. Czoli CD, Goniewicz M, Islam T, Kotnowski K, Hammond D. Consumer preferences for electronic cigarettes: results from a discrete choice experiment. Tob Control. 2016;25(e1):e30-36.

5. Farsalinos KE, Romagna G, Tsiapras D, Kyrzopoulos S, Spyrou A, Voudris V. Impact of flavour variability on electronic cigarette use experience: An internet survey. Int J Environ Res Public Health. 2013;10(12):7272-7282.

6. Tackett AP, Lechner WV, Meier E, et al. Biochemically verified smoking cessation and vaping beliefs among vape store customers. Addiction. 2015;110(5):868-874.

7. Carpenter CM, Wayne GF, Pauly JL, Koh HK, Connolly GN. New cigarette brands with flavors that appeal to youth: tobacco marketing strategies. Health Aff (Millwood). 2005;24(6):1601-1610.

8. Connolly GN. Sweet and spicy flavours: new brands for minorities and youth. Tob Control. 2004;13(3):211-212.

9. Kreslake JM, Wayne GF, Alpert HR, Koh HK, Connolly GN. Tobacco industry control of menthol in cigarettes and targeting of adolescents and young adults. Am J Public Health. 2008;98(9):1685-1692.

10. Lewis MJ, Wackowski O. Dealing with an innovative industry: a look at flavored cigarettes promoted by mainstream brands. Am J Public Health. 2006;96(2):244-251.

11. Morean ME, Butler ER, Bold KW, et al. Preferring more e-cigarette flavors is associated with e-cigarette use frequency among adolescents but not adults. PLoS One. 2018;13(1):e0189015.

12. Harrell MB, Weaver SR, Loukas A, et al. Flavored e-cigarette use: Characterizing youth, young adult, and adult users. Prev Med Reports. 2017;5:33-40.

13. St Helen G, Dempsey DA, Havel CM, Jacob P, 3rd, Benowitz NL. Impact of e-liquid flavors on nicotine intake and pharmacology of e-cigarettes. Drug Alcohol Depend. 2017;178:391-398.

14. Villanti AC, Johnson AL, Ambrose BK, et al. Flavored Tobacco Product Use in Youth and Adults: Findings From the First Wave of the PATH Study (2013-2014). Am J Prev Med. 2017;53(2):139-151.

15. Criscitelli K, Avena NM. The neurobiological and behavioral overlaps of nicotine and food addiction. Prev Med. 2016;92:82-89.

16. Berridge KC, Robinson TE, Aldridge JW. Dissecting components of reward: 'liking', 'wanting', and learning. Curr Opin Pharmacol. 2009;9(1):65-73.

17. Huang LL, Baker HM, Meernik C, Ranney LM, Richardson A, Goldstein AO. Impact of nonmenthol flavours in tobacco products on perceptions and use among youth, young adults and adults: a systematic review. Tob Control. 2017;26(6):709-719.

18. Noble AC, Arnold RA, Masuda, BM, Pecore SD, Schmidt JO, Stern PM. Progress towards a standardized system of wine aroma terminology. Am J Enol Vitic. 1984;35:107-109.

19. Lee KYM, Paterson A, Piggott JR. Origins of Flavour in Whiskies and a Revised Flavour Wheel: a Review. J Inst Brew. 2001;107(5):287-313.

20. Meilgaard MC, Dalgliesh CE, Clapperton JF. Beer Flavour Terminology. J Inst Brew. 1979;85(1):38- 
42.

21. Aparicio R, Morales MT, Alonso MV. Relationship between volatile compounds and sensory attributes of olive oils by the sensory wheel. Journal of the American Oil Chemists' Society. 1996;73(10):12531264.

22. Blommer Chocolate Company. Wheel of Flavor. 2009; http://www.blommer.com/_documents/ flavor-wheel-chocolate-copyright-2009.pdf. Accessed 8 September 2017.

23. Drake MA, Mcingvale SC, Gerard PD, Cadwallader KR, Civille GV. Development of a Descriptive Language for Cheddar Cheese. Journal of Food Science. 2001;66(9):1422-1427.

24. Specialty Coffee Association of America (SCAA), World Coffee Research Sensory Lexicon (WCR). Coffee Taster's Flavor Wheel. 2016; http:/www.scaa.org/?page=resources\&d=scaa-flavor-wheel. Accessed 8 September 2017.

25. Edwards M. Fragrances of the World - The Fragrance Wheel. 1992; http://www.fragrancesoftheworld. com/FragranceWheel. Accessed 8 September 2017.

26. Cigar Inspector. Cigar Flavors Wheel. 2013; http://www.cigarinspector.com/cigars-101/cigar-flavorcomponents. Accessed 8 September 2017.

27. Yingst JM, Veldheer S, Hammett E, Hrabovsky S, Foulds J. A Method for Classifying User-Reported Electronic Cigarette Liquid Flavors. Nicotine Tob Res. 2017;19(11):1381-1385.

28. VonVille H. Excel Workbook to Calculate Cohen's kappa for Screener Inter-rater Reliability. Houston: University of Texas School of Public Health Library; 2015.

29. McHugh ML. Interrater reliability: the kappa statistic. Biochem Med (Zagreb). 2012;22(3):276-282.

30. VonVille H. Excel Workbook for Screening Titles and Abstracts. Houston: University of Texas School of Public Health Library; 2015.

31. VonVille H. PRIMARY Excel Workbook for Systematic Reviews. Houston: University of Texas School of Public Health Library; 2017.

32. Noble AC, Arnold RA, Buechsenstein J, Leach EJ, Schmidt JO, Stern PM. Modification of a Standardized System of Wine Aroma Technology. Am J Enol Vitic. 1987;38(2):143-146.

33. Vasiljevic M, Petrescu DC, Marteau TM. Impact of advertisements promoting candy-like flavoured e-cigarettes on appeal of tobacco smoking among children: an experimental study. Tob Control. 2016;25(e2):e107-e112.

34. Berg CJ. Preferred flavors and reasons for e-cigarette use and discontinued use among never, current, and former smokers. Int J Public Health. 2016;61(2):225-236.

35. Chen Z, Zeng DD. Mining online e-liquid reviews for opinion polarities about e-liquid features. BMC Public Health. 2017;17(1):633.

36. Dawkins L, Turner J, Roberts A, Soar K. 'Vaping' profiles and preferences: an online survey of electronic cigarette users. Addiction. 2013;108(6):1115-1125.

37. Ford A, MacKintosh AM, Bauld L, Moodie C, Hastings G. Adolescents' responses to the promotion and flavouring of e-cigarettes. Int J Public Health. 2016;61(2):215-224.

38. Kim H, Lim J, Buehler SS, et al. Role of sweet and other flavours in liking and disliking of electronic cigarettes. Tob Control. 2016;25(Suppl 2):ii55-ii61.

39. Krishnan-Sarin S, Morean ME, Camenga DR, Cavallo DA, Kong G. E-cigarette use among high school and middle school adolescents in Connecticut. Nicotine Tob Res. 2015;17(7):810-818.

40. Litt MD, Duffy V, Oncken C. Cigarette smoking and electronic cigarette vaping patterns as a function of e-cigarette flavourings. Tob Control. 2016;25(Suppl 2):ii67-ii72. 
41. Oncken CA, Litt MD, McLaughlin LD, Burki NA. Nicotine concentrations with electronic cigarette use: Effects of sex and flavor. Nicotine Tob Res. 2014;17(4):473-478.

42. Pepper JK, Ribis1 KM, Brewer NT. Adolescents' interest in trying flavoured e-cigarettes. Tob Control. 2016;25(Suppl 2):ii62-ii66.

43. Piñeiro B, Correa JB, Simmons VN, et al. Gender differences in use and expectancies of e-cigarettes: Online survey results. Addictive Behaviors. 2016;52:91-97.

44. Shiplo S, Czoli CD, Hammond D. E-cigarette use in Canada: Prevalence and patterns of use in a regulated market. BMJ Open. 2015;5(8).

45. Wang L, Zhan Y, Li Q, Zeng DD, Leischow SJ, Okamoto J. An Examination of Electronic Cigarette Content on Social Media: Analysis of E-Cigarette Flavor Content on Reddit. Int J Environ Res Public Health. 2015;12(11):14916-14935.

46. Shiffman S, Sembower MA, Pillitteri JL, Gerlach KK, Gitchell JG. The Impact of Flavor Descriptors on Nonsmoking Teens' and Adult Smokers' Interest in Electronic Cigarettes. Nicotine Tob Res. 2015;17(10):1255-1262.

47. Soule EK, Lopez AA, Guy MC, Cobb CO. Reasons for using flavored liquids among electronic cigarette users: A concept mapping study. Drug Alcohol Depend. 2016;166:168-176.

48. Rosbrook K, Green BG. Sensory Effects of Menthol and Nicotine in an E-Cigarette. Nicotine Tob Res. 2016;18(7):1588-1595.

49. Cooper M, Harrell MB, Perez A, Delk J, Perry CL. Flavorings and Perceived Harm and Addictiveness of E-cigarettes among Youth. Tob Regul Sci. 2016;2(3):278-289.

50. Yingst JM, Veldheer S, Hrabovsky S, Nichols TT, Wilson SJ, Foulds J. Factors Associated With Electronic Cigarette Users' Device Preferences and Transition From First Generation to Advanced Generation Devices. Nicotine Tob Res. 2015;17(10):1242-1246.

51. Audrain-McGovern J, Strasser AA, Wileyto EP. The impact of flavoring on the rewarding and reinforcing value of e-cigarettes with nicotine among young adult smokers. Drug Alcohol Depend. 2016;166:263-267.

52. Chu KH, Unger JB, Cruz TB, Soto DW. Electronic Cigarettes on Twitter - Spreading the Appeal of Flavors. Tob Regul Sci. 2015;1(1):36-41.

53. The Senate and House of Representatives of the United States of America in Congress. Public Law 111-31: Family Smoking Prevention and Tobacco Control and Federal Retirement Reform. In: $111^{\text {th }}$ Congress; 2009:1776-1858.

54. Kamatou GP, Vermaak I, Viljoen AM, Lawrence BM. Menthol: a simple monoterpene with remarkable biological properties. Phytochemistry. 2013;96:15-25.

55. Hoffman AC, Salgado RV, Dresler C, Faller RW, Bartlett C. Flavour preferences in youth versus adults: a review. Tob Control. 2016;25(Suppl 2):ii32-ii39.

56. Finlayson G, Bordes I, Griffioen-Roose S, de Graaf C, Blundell JE. Susceptibility to overeating affects the impact of savory or sweet drinks on satiation, reward, and food intake in nonobese women. J Nutr. 2012;142(1):125-130.

57. The European Parliament and The Council of the European Union. Directive 2014/40/EU on the approximation of the laws, regulations and administrative provisions of the Member States concerning the manufacture, presentation and sale of tobacco and related products and repealing Directive 2001/37/EC. Vol 2014/40/EU: Official Journal of the European Union; 2014:127.

58. Feirman SP, Lock D, Cohen JE, Holtgrave DR, Li T. Flavored Tobacco Products in the United States: 
A Systematic Review Assessing Use and Attitudes. Nicotine Tob Res. 2016;18(5):739-749.

59. SCENIHR. Addictiveness and Attractiveness of Tobacco Additives. Brussels: European Union, 2010; https://ec.europa.eu/health/scientific_committees/emerging/docs/scenihr_o_029.pdf. 



\title{
CHAPTER 3
}

\section{Nearly 20000 e-liquids and 250 unique flavor descriptions: An overview of the Dutch market based on information from manufacturers}

\author{
Anne Havermans* \\ Erna J.Z. Krüsemann* \\ Jeroen L.A. Pennings \\ Kees de Graaf \\ Sanne Boesveldt \\ Reinskje Talhout \\ * shared first authorship
}

Published in Tobacco Control. 2021;30:57-62.

http://dx.doi.org/10.1136/tobaccocontrol-2019-055303. 


\begin{abstract}
Objectives: Flavors increase attractiveness of electronic cigarettes and stimulate use among vulnerable groups such as non-smoking adolescents. It is important for regulators to monitor the market to gain insight in, and regulate the range of e-liquid flavors that is available to consumers. E-liquid manufacturers are required to report key product information to authorities in the European Member States in which they plan to market their products. This information was used to provide an overview of e-liquid flavor descriptions marketed in the Netherlands in 2017. Methods: Two researchers classified 19266 e-liquids into the 16 main categories of the e-liquid flavor wheel, based on information from four variables in the European Common Entry Gate system. Flavor descriptions were further specified in subcategories. Results: For 16300 e-liquids (85\%), sufficient information was available for classification. The categories containing the highest number of e-liquids were fruit (34\%), tobacco (16\%), and dessert (10\%). For all e-liquids, excluding unflavored ones, 245 subcategories were defined within the main categories. In addition to previously reported subcategories, various miscellaneous flavors such as sandwich, buttermilk, and lavender were identified. Conclusions: In 2017, 20000 e-liquids were reported to be marketed in the Netherlands, in 245 unique flavor descriptions. The variety of marketed flavor descriptions reflects flavor preference of e-cigarette users as described in literature. Our systematic classification of e-liquids by flavor description provides a tool for organizing the huge variety in market supply, serves as an example for other countries to generate similar overviews, and can support regulators in developing flavor regulations.
\end{abstract}




\section{Introduction}

The use of electronic cigarettes (e-cigarettes) has risen significantly over the recent years ${ }^{1,2}$. Although e-cigarettes may provide a successful tool in smoking cessation ${ }^{3}$, concerns have been raised about initiation of e-cigarette use among young non-smokers ${ }^{4}$. An important factor in the high appeal of e-cigarettes to adolescents is the availability of a wide variety of e-liquid flavors ${ }^{5,6}$. Especially sweet and fruity flavors are appreciated by young users ${ }^{5,7-9}$. Not surprisingly, flavor descriptions play an important role in (online) e-cigarette promotion ${ }^{10-12}$. In line with this, research showed that flavor-related advertisements are appealing to youth, and trigger increased interest in, purchasing of, and use of e-cigarettes ${ }^{13-15}$. Unfortunately, it is difficult to monitor and control the purchase and use of e-cigarettes and e-liquids by young people, as these products are widely and readily available through tobacconists, vape shops, and particularly the internet ${ }^{16}$.

This raises concerns, as e-cigarette emissions may contain toxic chemicals that can be harmful to health ${ }^{17}$. In addition, a large proportion of available e-liquids contain the highly addictive compound nicotine. Teenagers and young adults are especially susceptible to develop addiction to nicotine, due to their ongoing brain maturation ${ }^{18}$. Because the vast range of flavored e-liquids is attractive to vulnerable consumer groups (e.g., adolescents and young adults), there is a clear need for regulation. Regulation of and research on e-liquid flavors can focus on consumer flavor perception (sensory science), flavoring ingredients that compose a perceived flavor (chemical analysis), and flavor descriptions that are used for marketing purposes. For example, current European and US regulations prohibit cigarettes and roll-your-own tobacco with a characterizing flavor ${ }^{19,20}$, which is monitored by a sensory panel of trained experts ${ }^{21,22}$. Recently, more and more countries also announced regulatory actions regarding e-cigarette flavors. For example, the US Food and Drug Administration (FDA) has announced regulation to limit sales of e-cigarettes to minors ${ }^{23}$ and to ban all e-cigarette flavors other than tobacco ${ }^{24}$. With the current study, we aim to support policy makers in regulating the marketing and promotion of e-liquids with flavor descriptions that may increase product interest and appeal.

In order to develop such regulation regarding the promotion of flavored e-liquids, it is important to monitor the market as to obtain a better understanding of the full range of products and flavors that are advertised to consumers. However, surveillance of the (online) e-cigarette marketplace can be challenging due to its rapidly changing and increasingly diverse character ${ }^{25}$. In addition, current estimations of available e-liquid flavors often rely on survey data ${ }^{5,7,26-28}$ rather than a complete census of products available on the market. Thus, at this moment, a complete overview of the supply of e-liquid flavor descriptions in any market worldwide is lacking.

However, according to the European Tobacco Product Directive ${ }^{19}$, e-cigarette and e-liquid manufacturers are required to provide key product information on the branding and composition of their products to authorities of the European Member States in which they plan to place their product on the market. This information provides a unique opportunity to establish an overview of the e-cigarette market in a particular European Member State. Here, we used e-liquid brand names and other flavor-related information as framed by the industry, which we refer to as "flavor descriptions" in this article (by others potentially colloquially referred to as "flavor names"). This means that we did not obtain sensory nor chemical data about (the perception or composition of) e-liquid flavors. Hence, this paper presents a comprehensive overview of flavor 
descriptions of e-liquids reported by manufacturers to be marketed in the Netherlands in 2017. 


\section{Methods}

\section{Data collection and preparation}

Flavor-related information about all products in the Dutch section of the European Common Entry Gate (EU-CEG) system ${ }^{29}$ was extracted on 30 November 2017. Only information from the category "refillable e-liquids and cartridges" (not "devices" or "individual parts") was selected for this study. When there were multiple presentations of the same product (e.g., one e-liquid marketed in different packages), one presentation was randomly selected. This was the case for 3922 products. Eight products were excluded due to incomplete information. E-liquids with the same flavor description, but different nicotine concentrations were considered different products and therefore separately included. The final dataset consisted of 19266 products.

There is no required field in the EU-CEG system ${ }^{29}$ to describe a product's flavor. However, information about a product's flavor can often be inferred from its brand name. In addition, some manufacturers provided a description of their product's flavor in the fields "Product Identification" and'/or "General Comment", which can be used optionally to provide additional product information. For our analyses, we therefore retrieved and combined all relevant flavor-related information from the following fields in the EU-CEG system: "Brand Name", "Brand Subtype Name", "Product Identification", and "General Comment". For example, all flavor-related information obtained from one (fictional) e-liquid could be "Dancer - Purple Blue Berry $-12 \mathrm{mg} / \mathrm{ml}$ ". This information was used for classification of e-liquids using the recently published e-liquid flavor wheel ${ }^{30}$. Thus, the flavor-related information that we retrieved from EU-CEG (brand names, information from other fields, or both) was reported by manufacturers to describe their product's flavor, and was recoded by us to a flavor description following a standardized approach (i.e., classification according to the flavor wheel).

In case insufficient flavor-related information was available in the database to directly classify an e-liquid ( $n=7116 ; 37 \%$ of total sample), a standardized internet search was conducted (www.google.com): input for the search was all flavor-related information of the e-liquid of interest plus the term "e-liquid". Resulting Web sites were consulted in consecutive order until a flavor description was found. E-liquids that were not found on the internet $(n=1680 ; 9 \%$ of total sample) were considered "unclassifiable". When the information from the EU-CEG system was too general to identify a specific e-liquid using the internet search (e.g. only referring to a brand or product range), the product was also considered unclassifiable.

In order to establish interrater reliability, two research assistants first independently classified a random sample of 166 e-liquids. The Cohen's Kappa R, calculated using R statistical software V.3.4.3, reached 0.86, which is considered a strong level of agreement ${ }^{31}$. Next, the same two research assistants each classified half of the total set of e-liquids. Finally, two of the authors checked the complete set for inconsistencies.

\section{Flavor classification}

E-liquids were classified in a consistent manner according to the e-liquid flavor wheel ${ }^{30}$. The flavor wheel consists of an inner wheel with 16 main categories: tobacco, menthol/mint, nuts, spices, coffee/tea, alcohol, other beverages, fruit-berries, fruit-citrus, fruit-tropical, fruit-other, dessert, candy, other sweets, other flavors, and unflavored. In the outer wheel, the main categories 
are further specified using subcategories. Because the subcategories of the published flavor wheel are not all encompassing, flavor descriptions that did not match the existing subcategories were regarded new subcategories. A detailed description of the classification approach can be found in Appendix A3.1.

\section{Data analyses}

The classified set of 19266 products was analyzed in R statistical software V.3.4.3. For each of the 16 main flavor categories, the following values were determined: the number of unique subcategories; the number of products (within each main and subcategory); the number of products with a secondary flavor description; the number of unique secondary flavor descriptions; and the average number of secondary flavor descriptions (within each main and subcategory).

Finally, the nicotine concentrations as declared by the industry were analyzed. E-liquids with a package unit other than one and a volume other than $10 \mathrm{~mL}$ were excluded from this analysis $(n=2427)$, because declared nicotine values of these products could not be related to a unit. E-liquids with unusual nicotine values that could not be related to a common nicotine unit $(n=359)$ or without data on their nicotine concentration at all $(n=1343)$ were excluded. This resulted in a total set of 12551 e-liquids for nicotine analysis. These e-liquids were divided into five groups according to their declared nicotine values, to represent the most common nicotine concentrations available on the Dutch market $(0,3,6,12$, and $18 \mathrm{mg} / \mathrm{mL})$. Most e-liquids contained a nicotine value that exactly matched these concentrations. Some values slightly deviated, therefore the following ranges were maintained: $0 \mathrm{mg} / \mathrm{mL}(\mathrm{n}=381), 3 \mathrm{mg} / \mathrm{mL}$ (range $>0$ and $<4.5 ; \mathrm{n}=2836$ ), $6 \mathrm{mg} / \mathrm{mL}$ (range $\geq 4.5$ and $<9 ; \mathrm{n}=3702), 12 \mathrm{mg} / \mathrm{mL}$ (range $\geq 9$ and $<$ $15 ; \mathrm{n}=3229$ ), and $18 \mathrm{mg} / \mathrm{mL}$ (range $\geq 15$ and $<20 ; \mathrm{n}=2403$ ). 


\section{Results}

\section{Primary flavor descriptors}

A total of 19266 e-liquids were submitted to the Dutch EU-CEG system on 30 November 2017. For 16300 (85\%) e-liquids, sufficient flavor-related information was available for classification of the product into one of the 16 main categories of the e-liquid flavor wheel ${ }^{30}$. Figure 3.1 shows the percentage of e-liquids classified within each of the main categories (i.e., reflecting a product's general flavor description). The categories containing the highest number of e-liquids were tobacco $(\mathrm{n}=2667 ; 16 \%)$, fruit-other $(\mathrm{n}=2506 ; 15 \%)$, fruit-berries $(\mathrm{n}=2164 ; 13 \%)$, and dessert $(\mathrm{n}=1710 ; 10 \%)$. Overall, $34 \%$ of the e-liquids were classified in one of the fruit categories (i.e., berries, citrus, tropical, or other fruit). The smallest category was other flavors (n $=169 ; 1 \%)$, followed by spices $(\mathrm{n}=176 ; 1 \%)$, nuts $(\mathrm{n}=179 ; 1 \%)$, and unflavored $(\mathrm{n}=266 ; 2 \%)$.

For each main category, except for unflavored, multiple subcategories (i.e., specific flavor descriptions) were defined, ranging from 4 (spices) to 46 (desserts). Overall, 245 unique subcategories were distinguished. Figure 3.2 shows the percentage of e-liquids within each subcategory, for each of the 16 main categories separately. All subcategories, as well as the number of e-liquids within each subcategory are reported in Appendix Table A3.2.
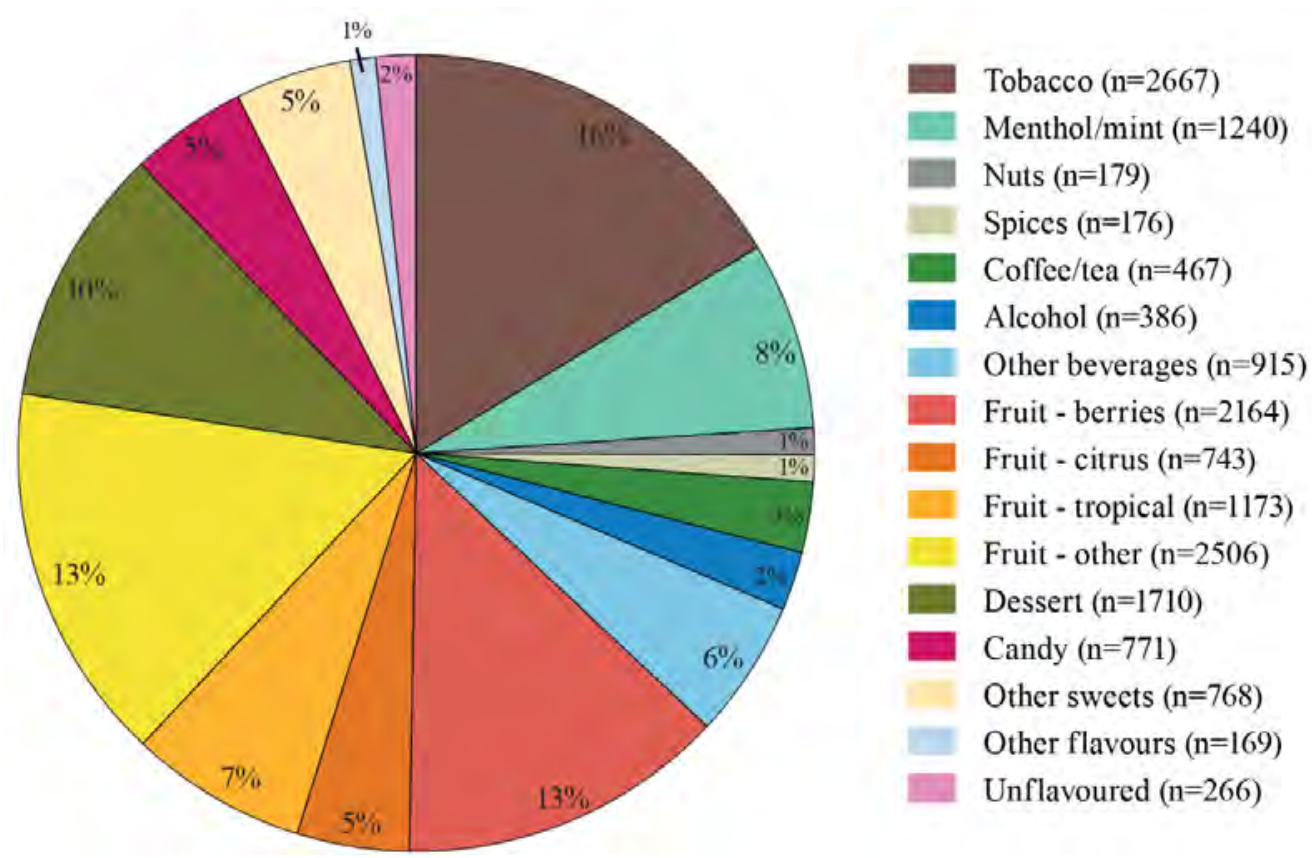

Figure 3.1: Distribution of the main flavor categories representing e-liquid availability on the Dutch market. Classification was based on brand names and flavor-related information as reported by manufacturers. Sequence and colors of the categories are based on the e-liquid flavor wheel ${ }^{30}$. 
"Other flavors" category

A total of 169 e-liquids were classified as other flavors, meaning that their primary flavor descriptor (based on brand name and flavor-related information from manufacturers) could not be classified into one of the other 15 main categories of the flavor wheel. Based on the products' flavor-related information, 16 subcategories were identified within the other flavors category. These subcategories were related to various flower and plant-related flavors (flowers, cherry blossom, roses, violet, lavender, hibiscus, honeysuckle, verbena, woodruff); vegetables (cucumber, rhubarb, fennel); bread-related flavors (bread, sandwich); and jam and cannabis.

\section{Secondary flavor descriptors}

For 5676 e-liquids (29\%), one or more secondary flavor descriptors could be distinguished (based on brand name and flavor-related information). Of the e-liquids containing at least one secondary flavor descriptor, the average number of secondary flavor descriptors was 1.6. The number of products containing secondary flavor descriptors was highest within the dessert category (66\%) and lowest within the menthol/mint category (17\%).

\section{Nicotine concentrations}

Nicotine concentrations ranged from 0 to $20 \mathrm{mg} / \mathrm{mL}$, which is the current legal maximum in the EU. Of the 12551 e-liquids that were included in the nicotine analysis, only $3 \%$ was reported to be nicotine-free $(0 \mathrm{mg} / \mathrm{mL})$. The percentage of e-liquids with high nicotine concentrations $(18 \mathrm{mg} /$ $\mathrm{mL}$ ) was highest within the unflavored category (40\%). Distribution of nicotine concentrations per main flavor category is visualized in Figure 3.3. 


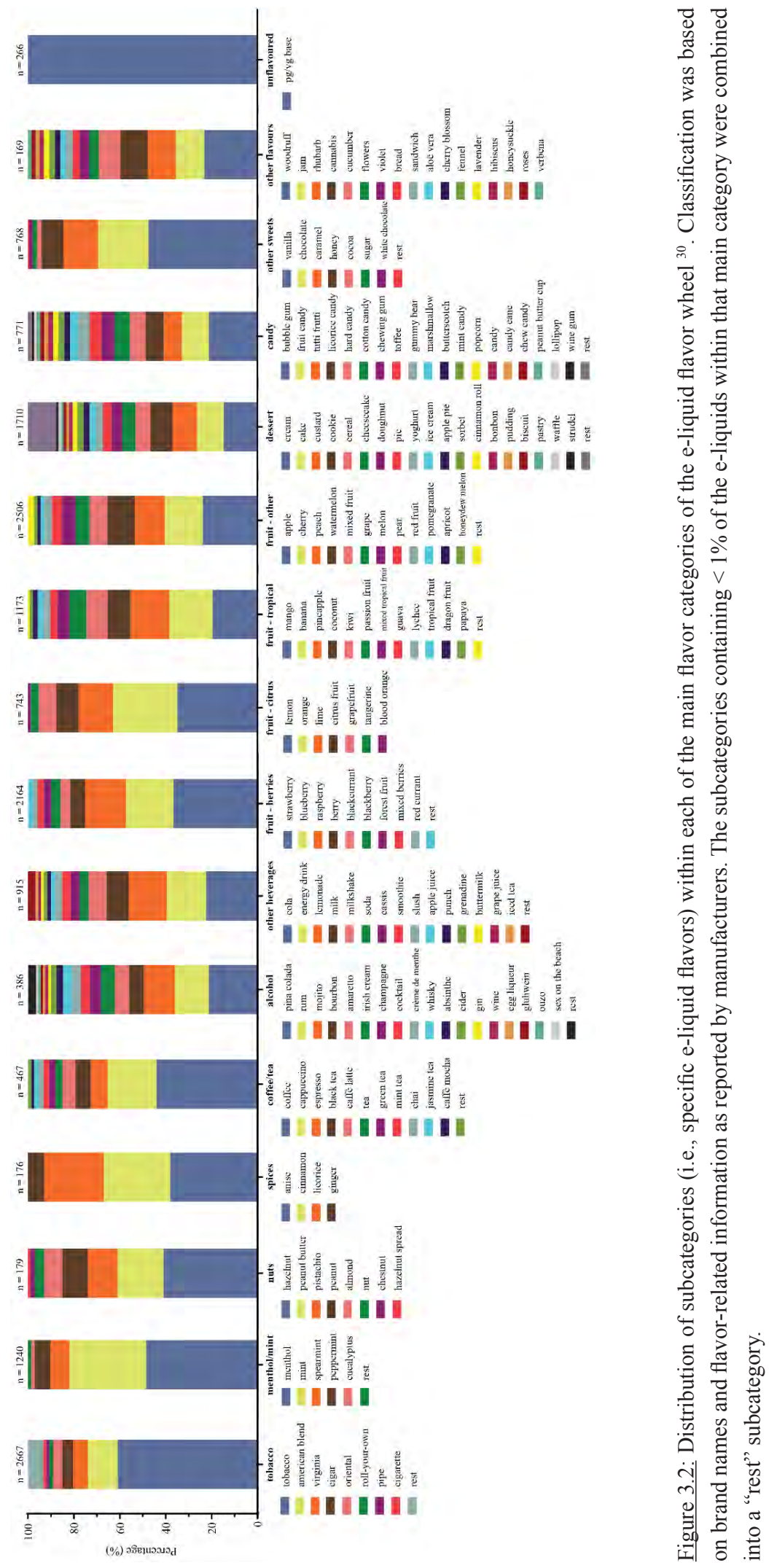




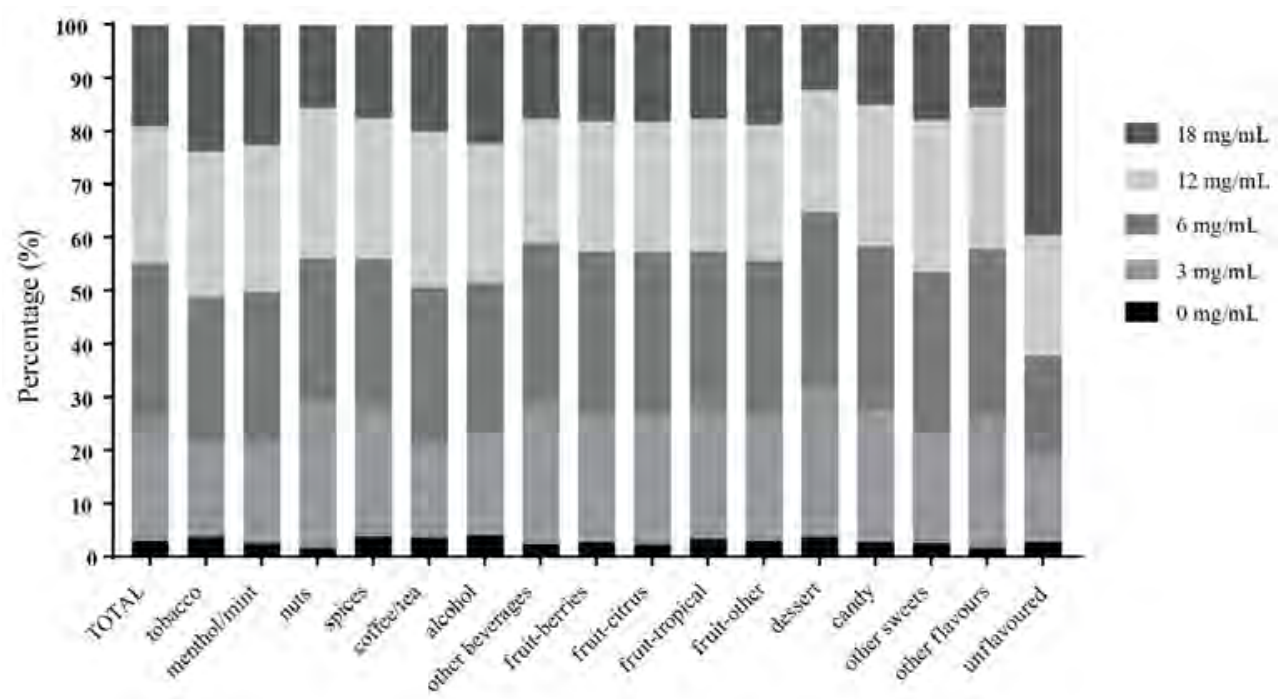

Figure 3.3: Nicotine concentrations within each of the main categories of the e-liquid flavor wheel ${ }^{30}$. Nicotine values reported by manufacturers in the EU-CEG system were categorized into five groups representing the most common nicotine concentrations available on the Dutch market. 


\section{Discussion}

This study presents a comprehensive overview of flavor descriptions of e-liquids reported to be marketed in the Netherlands in 2017. Using brand names and flavor-related information provided by manufacturers through the EU-CEG system ${ }^{29}$, e-liquids were classified into the main and subcategories of the e-liquid flavor wheel ${ }^{30}$. We found that $\sim 20000$ e-liquids were reported, having 245 unique flavor descriptions. Approximately one-third of the e-liquids was classified as having a fruit flavor (berries, citrus, tropical, or other fruits). Subsequently, the largest categories were tobacco, dessert, and menthol/mint. In line with this, literature shows that fruit and sweet flavors are the most commonly used flavors among both young and adult e-cigarette users $5,7,9,27,32$. Furthermore, adults who completely substituted the use of conventional cigarettes by e-cigarettes have often initiated e-cigarette use with fruity flavors rather than tobacco flavors, or switched from tobacco to non-tobacco e-liquid flavors over time ${ }^{7,28}$. On the other hand, dual users (using e-cigarettes as well as combustible tobacco) most commonly use tobacco-flavored e-liquids ${ }^{7,9,27}$, which is the second largest flavor category in the Netherlands based on the results of our current study. The flavor descriptions with which e-liquids are marketed, based on brand names and flavor-related information from manufacturers, thus seem to match flavor preferences as described in literature.

\section{Primary flavor descriptors}

Overall, at least $56 \%$ of the e-liquids was classified in a category that represents a sweet flavor (i.e., the ones classified as other beverages, fruit, dessert, candy, or other sweets). This large number of e-liquids marketed as sweet may be a response to sweet taste being the most preferred taste by all age groups ${ }^{33}$. Surprisingly, while umami, or savory, is typically also a popular taste in foods (e.g., broth, cooked meat, fish, and vegetables), not many e-liquids with a flavor description related to savory food products exist. It would be interesting for future research to investigate why the market for savory e-liquid flavors seems to be limited.

Within the main flavor categories, multiple subcategories were defined (245 in total, ranging from 4 to 46 per main category). However, regardless of the total number of subcategories within a particular main category, only four subcategories per main category were needed to classify roughly half of the e-liquids (Figure 3.2). For example, while the alcohol category contained 23 unique subcategories, more than half of all e-liquids in the alcohol main category were classified in the four largest subcategories: piña colada, rum, mojito, and bourbon. Similarly, while the dessert category contained 46 subcategories, almost half of all e-liquids in the dessert category were classified in the following four subcategories: cake, cookie, cream, custard. This shows that the main categories were dominated by only a few subcategories.

\section{Nicotine concentrations}

In the Netherlands, e-liquids are sold in nicotine concentrations ranging from 0 to $20 \mathrm{mg} / \mathrm{ml}$. The percentage of e-liquids with a high nicotine concentration (i.e., category of $18 \mathrm{mg} / \mathrm{mL}$ ) was relatively high in the unflavored category, as compared to the other main categories of the flavor wheel. A reason for this may be that unflavored e-liquids, some of them marketed as "nicotine booster", are mainly purchased to add nicotine to hand-made e-liquid mixes. Finally, only 3\% 
of the e-liquids were marketed as nicotine-free $(0 \mathrm{mg} / \mathrm{mL})$. However, we excluded e-liquids without any data on their nicotine concentration, while manufacturers of nicotine-free e-liquids may purposely not have submitted nicotine-related information for these e-liquids. Also, in the Netherlands, nicotine-free e-liquids were not required to be registered in the EU-CEG system at the time of this study ${ }^{34}$. Therefore, our results may provide an underestimation of the actual number of nicotine-free e-liquids on the Dutch market.

\section{Limitations}

For this study, we used flavor-related information retrieved from a set of variables in the EU-CEG system (i.e., "Brand Name", "Brand Subtype Name", "Product Identification", and "General Comment") ${ }^{29}$. This information was submitted by manufacturers, and does not necessarily represent the flavor as perceived by consumers or the flavor descriptions used for marketing on Web shops. Because of limited or unspecific flavor-related information from EUCEG, classification of approximately one-third of the e-liquids required an internet search, and eventually, $15 \%$ of the e-liquids could not be classified in any of the flavor wheel categories. Some of these products may have been removed from the market in the period between data extraction and the time of data analysis. Accordingly, it should be noted that information retrieved from the EU-CEG system represents a snapshot of the market on a single day. In addition, as products should be notified in the EU-CEG system at least 6 months prior to being placed on the market, it is possible that some products submitted to the EU-CEG system were not actually on the market at the time of data extraction.

Importantly, as information in the EU-CEG is provided by the e-cigarette industry, without the aim of sustaining research, it should be treated with appropriate caution. In order to verify and support conclusions based on EU-CEG data, independent market research may be conducted. After all, strict surveillance of submitted industry data is needed to ensure an accurate dataset for future use. A more elaborate discussion on the limitations (and strengths) of the data source and approach to flavor classification can be found in Appendix A3.3.

\section{Policy recommendations}

The fact that e-liquids are marketed with such a large variety of - especially sweet - flavor descriptions is highly concerning in the light of previous research demonstrating the great appeal of such flavors to youth, and therefore underlines a significant need for regulation. That is, in order to reduce e-cigarette appeal for adolescents and young adults, the abundant landscape of flavors in which e-liquids are promoted should be restricted. This can be achieved, for instance, by only allowing e-liquid flavors to be described as one of the 16 general terms that make up the main categories of the flavor wheel (inner wheel). Regulating e-liquid flavor names to the actual 16 categories also prevents the marketing of products with extraordinary names that may be particularly appealing to youth, such as unicorn-themed names ${ }^{32}$. Taking potential flavor regulation a step further, specific flavor categories that are proven to be particularly attractive to vulnerable user groups (i.e., non-smokers and youth), such as candy and dessert flavors, could be banned completely. Such forms of regulation mostly target the way e-liquids are marketed, and not the actual perceived flavor or composition of the products, which will make enforcement more feasible. However, the potential negative effect of implementing such rules on smokers aiming to 
switch towards exclusive e-cigarette use and thereby quit smoking should be considered as well.

\section{Conclusions}

This study was the first to use industry data to classify marketed e-liquids by flavor descriptions using brand names and flavor-related information. We showed that, in 2017, the Dutch market comprised $\sim 20000$ e-liquids in 245 unique flavor descriptions. One-third of the (classifiable) e-liquids was marketed as having a fruit flavor, and over half of the e-liquids as having any type of sweet flavor. The marketed variety of e-liquid flavor descriptions as identified in this study reflects flavor preferences of e-cigarette users as described in previous literature. Our approach can serve as an example for other countries to generate a similar overview. This is especially relevant for European Member States as they have the opportunity to retrieve a similar dataset from the EU-CEG system. This allows comparing the landscape of advertised e-liquid flavor descriptions between local markets. Furthermore, our systematic classification of e-liquids by flavor description provides structure in the huge variety in market supply, and can serve as a tool for policy makers in developing rules for e-liquid flavor regulation.

\section{What this paper adds}

What is already known on this subject: A main reason for the high appeal of e-cigarettes to young people is the availability of a wide variety of, especially sweet, e-liquid flavors. Since e-liquids are widely and readily available to young people, and may be harmful to health, there is a clear need for flavor regulation. What important gaps in knowledge exist on this topic: At this time, regulators do not have a complete overview of available e-liquids and flavor descriptions on their regional markets. This information is needed in order to monitor, and potentially regulate, the increasingly large and diversifying e-cigarette market. What this paper adds: This study was the first to use industry data to classify marketed e-liquids into the categories of the e-liquid flavor wheel using flavor-related information from manufacturers. Classifying e-liquids by flavor description helps to focus regulation on flavor categories that are, for example, most attractive to specific consumer groups and/or particularly contain toxic flavorings. Our approach can serve as an example for other regional markets to perform similar analyses. 


\section{Appendix}

Appendix A3.1: A detailed description of the classification approach

E-liquids were classified in a consistent manner according to our recently developed e-liquid flavor wheel

${ }^{30}$. The flavor wheel consists of an inner wheel with 16 main categories: tobacco, menthol/mint, nuts, spices, coffee/tea, alcohol, other beverages, fruit-berries, fruit-citrus, fruit-tropical, fruit-other, dessert, candy, other sweets, other flavors, and unflavored. In the outer wheel, the main categories are further specified using subcategories. Because the subcategories of the published flavor wheel are not all-encompassing, flavor descriptions that did not match the existing subcategories were regarded new subcategories.

Following the approach described in the flavor wheel publication ${ }^{30}$, primary and secondary e-liquid flavor descriptors were distinguished. An e-liquid's primary flavor descriptor was defined as the flavor descriptor associated with a particular (food or other) product as a whole (e.g., "piña colada" or "bubble gum"). Primary flavor descriptors were classified into one of the 16 main categories (inner wheel) and in one of the subcategories (outer wheel). Thus, e-liquids with piña colada as primary flavor descriptor would be classified in the main alcohol category and in the piña colada subcategory.

If the flavor description does not relate to one product as a whole, but contains several separate attributes (e.g., "strawberry with a hint of menthol and kiwi"), the e-liquid contains secondary flavor descriptors. In such case, the first flavor mentioned was considered the primary flavor descriptor (strawberry) and any other attributes were secondary flavor descriptors (menthol and kiwi). Secondary descriptors, if present, were classified into a subcategory only (outer wheel).

Subcategories were not allowed to encompass brand names (e.g. "skittles" or "red bull"), tastes (sweet, sour, bitter, salt, umami), or colors. Information regarding a specific country or area was not used for classification, unless it represented a well-known product type such as American tobacco. Along the classification process, the terms "ice" or "iced" in a brand name appeared to be used to describe a fresh, menthol flavor. Therefore, e-liquids referred to with these terms were consistently classified as having a secondary menthol flavor. 
Appendix Table A3.2: The number of e-liquids within each of the 245 defined subcategories within the 16 main flavor categories.

\begin{tabular}{|c|c|c|c|c|}
\hline $\begin{array}{l}\text { Main flavor } \\
\text { category }\end{array}$ & Subcategories I & $\begin{array}{l}\text { Number of } \\
\text { e-liquids I }\end{array}$ & Subcategories II & $\begin{array}{l}\text { Number of } \\
\text { e-liquids II }\end{array}$ \\
\hline \multirow{10}{*}{$\begin{array}{l}\text { Tobacco } \\
(\mathrm{n}=2667)\end{array}$} & tobacco & 1624 & black tobacco & 23 \\
\hline & American blend & 347 & flue-cured & 23 \\
\hline & Virginia & 175 & cavendish & 20 \\
\hline & cigar & 119 & Kentucky & 19 \\
\hline & oriental & 109 & latakia & 11 \\
\hline & roll-your-own & 44 & kretek & 10 \\
\hline & pipe & 38 & cigarillo & 8 \\
\hline & cigarette & 36 & Arabian blend & 7 \\
\hline & burley & 27 & snuff & 1 \\
\hline & shisha & 26 & & \\
\hline \multirow{4}{*}{$\begin{array}{l}\text { Menthol/mint } \\
(\mathrm{n}=1240)\end{array}$} & menthol & 601 & eucalyptus & 19 \\
\hline & $\operatorname{mint}$ & 415 & wintergreen & 12 \\
\hline & spearmint & 103 & horehound & 6 \\
\hline & peppermint & 84 & & \\
\hline \multirow{4}{*}{$\begin{array}{l}\text { Nuts } \\
(\mathrm{n}=179)\end{array}$} & hazelnut & 74 & almond & 14 \\
\hline & peanut butter & 35 & nut & 8 \\
\hline & pistachio & 23 & chestnut & 3 \\
\hline & peanut & 19 & hazelnut spread & 3 \\
\hline \multirow{2}{*}{$\begin{array}{l}\text { Spices } \\
(\mathrm{n}=176)\end{array}$} & anise & 67 & licorice & 46 \\
\hline & cinnamon & 51 & ginger & 12 \\
\hline \multirow{7}{*}{$\begin{array}{l}\text { Coffee/tea } \\
(\mathrm{n}=467)\end{array}$} & coffee & 206 & mint tea & 11 \\
\hline & cappuccino & 99 & chai & 10 \\
\hline & espresso & 34 & jasmine tea & 10 \\
\hline & black tea & 31 & caffè mocha & 5 \\
\hline & caffè latte & 27 & earl grey tea & 4 \\
\hline & tea & 14 & medina tea & 3 \\
\hline & green tea & 13 & & \\
\hline \multirow{12}{*}{$\begin{array}{l}\text { Alcohol } \\
(\mathrm{n}=386)\end{array}$} & piña colada & 82 & gin & 5 \\
\hline & rum & 57 & wine & 5 \\
\hline & mojito & 52 & egg liqueur & 4 \\
\hline & bourbon & 25 & gluhwein & 4 \\
\hline & amaretto & 24 & ouzo & 4 \\
\hline & Irish cream & 24 & sex on the beach & 4 \\
\hline & champagne & 18 & blue curacao & 3 \\
\hline & cocktail & 15 & eggnog & 3 \\
\hline & crème de menthe & 15 & malibu & 3 \\
\hline & whisky & 15 & crème de cassis & 2 \\
\hline & absinthe & 11 & gin tonic & 2 \\
\hline & cider & 9 & & \\
\hline
\end{tabular}




\begin{tabular}{|c|c|c|c|c|}
\hline \multirow{12}{*}{$\begin{array}{l}\text { Other beverages } \\
(\mathrm{n}=915)\end{array}$} & cola & 204 & buttermilk & 11 \\
\hline & energy drink & 157 & grape juice & 11 \\
\hline & lemonade & 152 & iced tea & 11 \\
\hline & milk & 89 & tropical juice & 6 \\
\hline & milkshake & 71 & chocolate milk & 5 \\
\hline & soda & 37 & fruit drink & 5 \\
\hline & cassis & 35 & watermelon juice & 4 \\
\hline & smoothie & 34 & orange juice & 3 \\
\hline & slush & 27 & tonic & 3 \\
\hline & apple juice & 17 & cactus juice & 2 \\
\hline & punch & 15 & root beer & 2 \\
\hline & grenadine & 14 & & \\
\hline \multirow{8}{*}{$\begin{array}{l}\text { Fruit - berries } \\
(\mathrm{n}=2164)\end{array}$} & strawberry & 795 & red currant & 44 \\
\hline & blueberry & 448 & cranberry & 15 \\
\hline & raspberry & 383 & waterberry & 8 \\
\hline & berry & 141 & boysenberry & 7 \\
\hline & blackcurrant & 94 & loganberry & 7 \\
\hline & blackberry & 87 & dewberry & 4 \\
\hline & forest fruit & 69 & gooseberry & 4 \\
\hline & mixed berries & 58 & & \\
\hline \multirow{4}{*}{$\begin{array}{l}\text { Fruit }- \text { citrus } \\
(\mathrm{n}=743)\end{array}$} & lemon & 259 & grapefruit & 58 \\
\hline & orange & 209 & tangerine & 25 \\
\hline & lime & 111 & blood orange & 10 \\
\hline & citrus fruit & 71 & & \\
\hline \multirow{7}{*}{$\begin{array}{l}\text { Fruit - tropical } \\
(\mathrm{n}=1173)\end{array}$} & mango & 229 & guava & 36 \\
\hline & banana & 222 & lychee & 35 \\
\hline & pineapple & 198 & tropical fruit & 29 \\
\hline & coconut & 116 & dragon fruit & 25 \\
\hline & kiwi & 108 & papaya & 16 \\
\hline & passion fruit & 88 & pawpaw & 10 \\
\hline & mixed tropical fruit & 61 & & \\
\hline \multirow{10}{*}{$\begin{array}{l}\text { Fruit }- \text { other } \\
(\mathrm{n}=2506)\end{array}$} & apple & 597 & apricot & 36 \\
\hline & cherry & 415 & honeydew melon & 34 \\
\hline & peach & 327 & fruit & 17 \\
\hline & watermelon & 299 & raisin & 13 \\
\hline & mixed fruit & 195 & fig & 10 \\
\hline & grape & 154 & plum & 10 \\
\hline & melon & 151 & nectarine & 8 \\
\hline & pear & 108 & cactus & 7 \\
\hline & red fruit & 82 & star fruit & 3 \\
\hline & pomegranate & 40 & & \\
\hline
\end{tabular}




\begin{tabular}{|c|c|c|c|c|}
\hline \multirow{23}{*}{$\begin{array}{l}\text { Dessert } \\
(\mathrm{n}=1710)\end{array}$} & cream & 251 & churros & 12 \\
\hline & cake & 198 & muffin & 12 \\
\hline & custard & 183 & rice crisps & 12 \\
\hline & cookie & 166 & bavarian cream & 11 \\
\hline & cereal & 107 & crème brûlée & 11 \\
\hline & cheesecake & 101 & nougat & 11 \\
\hline & donut & 80 & baked apple & 9 \\
\hline & pie & 64 & smore & 9 \\
\hline & yoghurt & 53 & cannoli & 8 \\
\hline & ice cream & 46 & rice pudding & 8 \\
\hline & apple pie & 44 & crêpe & 7 \\
\hline & sorbet & 44 & red velvet cake & 7 \\
\hline & cinnamon roll & 38 & cereal bar & 5 \\
\hline & bonbon & 25 & dulce de leche & 5 \\
\hline & pudding & 21 & gingerbread & 5 \\
\hline & biscuit & 20 & crème anglais & 4 \\
\hline & pastry & 20 & croissant & 4 \\
\hline & waffle & 19 & macaron & 3 \\
\hline & strudel & 17 & scone & 3 \\
\hline & dough & 16 & tiramisu & 3 \\
\hline & pancake & 15 & tompouce & 3 \\
\hline & merengue & 14 & praline & 2 \\
\hline & cupcake & 13 & galaktoboureko & 1 \\
\hline \multirow{11}{*}{$\begin{array}{l}\text { Candy } \\
(\mathrm{n}=771)\end{array}$} & bubble gum & 163 & mint candy & 19 \\
\hline & fruit candy & 90 & popcorn & 18 \\
\hline & tutti frutti & 62 & candy & 16 \\
\hline & licorice candy & 61 & candy cane & 14 \\
\hline & hard candy & 53 & chew candy & 13 \\
\hline & cotton candy & 51 & peanut butter cup & 12 \\
\hline & chewing gum & 45 & lollipop & 9 \\
\hline & toffee & 39 & wine gum & 8 \\
\hline & gummy bear & 38 & marzipan & 5 \\
\hline & marshmallow & 27 & Turkish delight & 5 \\
\hline & butterscotch & 20 & jawbreaker & 3 \\
\hline \multirow{4}{*}{$\begin{array}{l}\text { Other sweets } \\
(\mathrm{n}=768)\end{array}$} & vanilla & 364 & cocoa & 15 \\
\hline & chocolate & 170 & sugar & 15 \\
\hline & caramel & 115 & white chocolate & 11 \\
\hline & honey & 73 & mocha & 5 \\
\hline
\end{tabular}




\begin{tabular}{lllll}
\hline $\begin{array}{l}\text { Other flavors } \\
(\mathrm{n}=169)\end{array}$ & woodruff & 39 & aloë vera & 4 \\
& jam & 21 & cherry blossom & 4 \\
& rhubarb & 21 & fennel & 4 \\
& cannabis & 20 & lavender & 4 \\
& cucumber & 16 & hibiscus & 3 \\
& flowers & 7 & honeysuckle & 3 \\
& violet & 7 & roses & 3 \\
& bread & 5 & verbena & 3 \\
& sandwich & 5 & & \\
\hline Unflavored & PG/VG base & 266 & & \\
\hline
\end{tabular}

The main flavor categories were based on our previously published e-liquid flavor wheel ${ }^{30}$. PG, propylene glycol; VG, vegetable glycerin. 
Appendix A3.3: A discussion on the strengths and limitations of the data source and approach to flavor classification.

The EU-CEG system provides access to an extensive collection of information regarding all tobacco products and e-cigarettes that have been marketed in each European Member State. Such information provides a unique opportunity to obtain insight in various aspects of the European e-cigarette and tobacco product market. For instance, it allows analyses on branding, ingredients, and emissions of products that are currently on the market as well as of products that have been removed from the market. In this way, trends in tobacco products and e-cigarettes can be monitored, such as the emergence of new products or changes in composition as a consequence of new regulations. Therefore, the EU-CEG system can be a highly valuable tool in monitoring the market, provided that manufacturers consistently and regularly update the data on their products. This is especially important in a constantly changing and expanding market, as the one for e-cigarettes.

It should be noted that marketing-related information that could be used to determine an e-liquid's primary flavor descriptor may differ between the EU-CEG system and Web shops, and across Web shops. E-liquids currently classified using information from the EU-CEG system may therefore have been classified in a different main flavor category if they were to be searched for online. In addition, the primary flavor descriptor that was used for classification may not be a complete representation of an e-liquid's flavor. This is due to fact that only the first flavor descriptor mentioned was selected for classification of e-liquids containing multiple flavor descriptors, because e-liquids can be classified in only one main category. Thus, for a careful interpretation of results, it should be taken into account that classification of e-liquids by marketed flavor descriptions depends on the source of information.

Moreover, as flavor-related information from the EU-CEG system is limited, the number of secondary flavor descriptors may have been underestimated. Information about e-liquids that were described with at least one secondary flavor descriptor (approximately 30\%), was mostly obtained using the internet search. If flavor descriptions of more e-liquids were to be searched for online or if the EUCEG system would contain more extensive flavor-related information, we expect the number of secondary flavor descriptors to be higher.

In this study, the e-liquid flavor wheel was used and proved to be a useful tool for classification of e-liquids by flavor description. Future research using e-liquid flavor categories may consider to more precisely structure the dessert category, as it contained many different flavor descriptions, varying from pastries (e.g., cinnamon roll, croissant, doughnut), cakes, and pies to dairy flavors (e.g., yoghurt, [ice] cream, pudding) and treats (e.g., praline, cookies, nougat). For example, a sub-classification by type of dessert could be included (similar to the division of the fruit category into berries, citrus, tropical, and other types of fruits), or separate main categories representing these different types of flavors could be created. Furthermore, some dessert-related flavor descriptions could be classified in new main categories that could also be used to classify certain flavors that were part of the other flavors category in this study. For instance, flavor descriptions such as sandwich and bread could be combined with cereal from the dessert category into a new main grains-related category. Additional main categories related to plants, flowers, and/or vegetables could be considered as well. 


\section{References}

1. Filippidis FT, Laverty AA, Gerovasili V, Vardavas CI. Two-year trends and predictors of e-cigarette use in 27 European Union member states. Tob Control. 2017;26(1):98.

2. McMillen RC, Gottlieb MA, Shaefer RM, Winickoff JP, Klein JD. Trends in Electronic Cigarette Use Among U.S. Adults: Use is Increasing in Both Smokers and Nonsmokers. Nicotine Tob Res. 2015;17(10):1195-1202.

3. Rahman MA, Hann N, Wilson A, Mnatzaganian G, Worrall-Carter L. E-cigarettes and smoking cessation: evidence from a systematic review and meta-analysis. PLoS One. 2015;10(3):e0122544.

4. Soneji S, Barrington-Trimis JL, Wills TA, et al. Association Between Initial Use of e-Cigarettes and Subsequent Cigarette Smoking Among Adolescents and Young Adults: A Systematic Review and Meta-analysis. JAMA Pediatr. 2017;171(8):788-797.

5. Schneller LM, Bansal-Travers M, Goniewicz ML, McIntosh S, Ossip D, O'Connor RJ. Use of flavored electronic cigarette refill liquids among adults and youth in the US-Results from Wave 2 of the Population Assessment of Tobacco and Health Study (2014-2015). PLoS One. 2018;13(8):e0202744.

6. Villanti AC, Johnson AL, Ambrose BK, et al. Flavored Tobacco Product Use in Youth and Adults: Findings From the First Wave of the PATH Study (2013-2014). Am J Prev Med. 2017;53(2):139-151.

7. Harrell MB, Weaver SR, Loukas A, et al. Flavored e-cigarette use: Characterizing youth, young adult, and adult users. Prev Med Reports. 2017;5:33-40.

8. Pepper JK, Ribisl KM, Brewer NT. Adolescents' interest in trying flavoured e-cigarettes. Tob Control. 2016;25(Suppl 2):ii62-ii66.

9. Zare S, Nemati M, Zheng Y. A systematic review of consumer preference for e-cigarette attributes: Flavor, nicotine strength, and type. PLoS One. 2018;13(3):e0194145.

10. Liang Y, Zheng X, Zeng DD, Zhou X. Impact of Flavor on Electronic Cigarette Marketing in Social Media. 2016. In: Zheng X., Zeng D., Chen H., Leischow S. (eds) Smart Health. ICSH 2015. Lecture Notes in Computer Science, vol 9545. Springer, Cham.

11. Grana RA, Ling PM. "Smoking Revolution": A Content Analysis of Electronic Cigarette Retail Websites. Am J Prev Med. 2014;46(4):395-403.

12. Soule EK, Sakuma KK, Palafox S, et al. Content analysis of internet marketing strategies used to promote flavored electronic cigarettes. Addict Behav. 2019;91:128-135.

13. Garrison KA, O'Malley SS, Gueorguieva R, Krishnan-Sarin S. A fMRI study on the impact of advertising for flavored e-cigarettes on susceptible young adults. Drug Alcohol Depend. 2018;186:233241.

14. Vasiljevic M, Petrescu DC, Marteau TM. Impact of advertisements promoting candy-like flavoured e-cigarettes on appeal of tobacco smoking among children: an experimental study. Tob Control. 2016;25(e2):e107-e112.

15. Farrelly MC, Duke JC, Crankshaw EC, et al. A Randomized Trial of the Effect of E-cigarette TV Advertisements on Intentions to Use E-cigarettes. Am J Prev Med. 2015;49(5):686-693.

16. Zhu S-H, Sun JY, Bonnevie E, et al. Four hundred and sixty brands of e-cigarettes and counting: implications for product regulation. Tob Control. 2014;23(suppl 3):iii3-iii9.

17. Hutzler C, Paschke M, Kruschinski S, Henkler F, Hahn J, Luch AJAoT. Chemical hazards present in liquids and vapors of electronic cigarettes. Arch Toxicol. 2014;88(7):1295-1308.

18. England LJ, Bunnell RE, Pechacek TF, Tong VT, McAfee TA. Nicotine and the Developing Human: A Neglected Element in the Electronic Cigarette Debate. Am J Prev Med. 2015;49(2):286-293. 
19. The European Parliament and The Council of the European Union. Directive 2014/40/EU on the approximation of the laws, regulations and administrative provisions of the Member States concerning the manufacture, presentation and sale of tobacco and related products and repealing Directive 2001/37/EC. Vol 2014/40/EU: Official Journal of the European Union; 2014:127.

20. The Senate and House of Representatives of the United States of America in Congress. Public Law 111-31: Family Smoking Prevention and Tobacco Control and Federal Retirement Reform. In: $111^{\text {th }}$ Congress; 2009:1776-1858.

21. Chambers E, Paschke T. Validation of a recommended practice for assessing "characterizing flavor" to meet requirements of the EU Tobacco Product Directive (2014/40/EU). J Sens Stud. 2019;34(5):e12511.

22. Krusemann EJZ, Lasschuijt MP, de Graaf C, et al. Sensory analysis of characterising flavours: evaluating tobacco product odours using an expert panel. Tob Control. 2019;28(2):152-160.

23. US Department of Health and Human Services. Modifications to Compliance Policy for Certain Deemed Tobacco Products In: Products CfT, ed. FDA-2019-D-0661 Rockville, MD: Food and Drug Administration; 2019.

24. US Food and Drug Administration (FDA). Trump Administration Combating Epidemic of Youth E-Cigarette Use with Plan to Clear Market of Unauthorized, Non-Tobacco-Flavored E-Cigarette Products [press release]. Silver Spring, MD, 2019.

25. Giovenco DP, Hammond D, Corey CG, Ambrose BK, Delnevo CD. E-Cigarette Market Trends in Traditional U.S. Retail Channels, 2012-2013. Nicotine Tob Res. 2015;17(10):1279-1283.

26. O'Connor RJ, Fix BV, McNeill A, et al. Characteristics of nicotine vaping products used by participants in the 2016 ITC Four Country Smoking and Vaping Survey. Addiction. 2019;114 Suppl $1: 15-23$.

27. Berg CJ. Preferred flavors and reasons for e-cigarette use and discontinued use among never, current, and former smokers. Int J Public Health. 2016;61(2):225-236.

28. Russell C, McKeganey N, Dickson T, Nides M. Changing patterns of first e-cigarette flavor used and current flavors used by 20,836 adult frequent e-cigarette users in the USA. Harm Reduct J. 2018;15(1):33.

29. European Commission. EU Common Entry Gate (EU-CEG). 2016; https://ec.europa.eu/health/ euceg/introduction_en.

30. Krüsemann EJZ, Boesveldt S, de GraafK, Talhout R. An E-Liquid Flavor Wheel: A Shared Vocabulary Based on Systematically Reviewing E-Liquid Flavor Classifications in Literature. Nicotine Tob Res. 2019;21(10):1310-1319.

31. McHugh ML. Interrater reliability: the kappa statistic. Biochem Med (Zagreb). 2012;22(3):276-282.

32. Farsalinos KE, Romagna G, Tsiapras D, Kyrzopoulos S, Spyrou A, Voudris V. Impact of flavour variability on electronic cigarette use experience: An internet survey. Int J Environ Res Public Health. 2013;10(12):7272-7282.

33. Hoffman AC, Salgado RV, Dresler C, Faller RW, Bartlett C. Flavour preferences in youth versus adults: a review. Tob Control. 2016;25(Suppl 2):ii32-ii39.

34. Regeling van de Staatssecretaris van Volksgezondheid, Welzijn en Sport van 23 april 2018, kenmerk 1220157-166930-WJZ, houdende wijziging van de Tabaks- en rookwarenregeling ter regeling van de elektronische sigaret zonder nicotine en nadere regeling van voor roken bestemde kruidenproducten en nadere verpakkingseisen voor roken bestemde tabaksproducten Staatscourant van het Koninkrijk 
der Nederlanden; 2018.

76 Chapter 3. Overview of the Dutch E-liquid Market 



\section{CHAPTER 4}

\section{A comprehensive overview of common e-liquid ingredients and how they can be used to predict an e-liquid's flavor category}

Erna J.Z. Krüsemann*

Anne Havermans*

Jeroen L.A. Pennings

Kees de Graaf

Sanne Boesveldt

Reinskje Talhout

* shared first authorship

Published in Tobacco Control. E-publication ahead of print: 10 February 2020. http://dx.doi.org/10.1136/tobaccocontrol-2019-055447. 


\begin{abstract}
Objectives: Flavors increase e-cigarette attractiveness and use, and thereby exposure to potentially toxic ingredients. An overview of e-liquid ingredients is needed to select target ingredients for chemical-analytical and toxicological research and for regulatory approaches aimed at reducing e-cigarette attractiveness. Using information from e-cigarette manufacturers, we aim to identify the flavoring ingredients most frequently added to e-liquids on the Dutch market. Additionally, we used flavoring compositions to automatically classify e-liquids into flavor categories, thereby generating an overview that can facilitate market surveillance. Methods: We used a dataset containing 16839 e-liquids that were manually classified into 16 flavor categories in our previous study. For the overall set and each flavor category, we identified flavorings present in more than $10 \%$ of the products and their median quantities. Next, quantitative and qualitative ingredient information was used to predict e-liquid flavor categories using a random forest algorithm. Results: We identified 219 unique ingredients that were added to more than 100 e-liquids, of which 213 were flavorings. The mean number of flavorings per e-liquid was $10 \pm 15$. The most frequently used flavorings were vanillin (present in $35 \%$ of all liquids), ethyl maltol (32\%) and ethyl butyrate (28\%). In addition, we identified 29 category-specific flavorings. Moreover, e-liquids' flavor categories were predicted with an overall accuracy of $70 \%$. Conclusions: Information from manufacturers can be used to identify frequently used and category-specific flavorings. Qualitative and quantitative ingredient information can be used to successfully predict an e-liquid's flavor category, serving as an example for regulators that have similar datasets available.
\end{abstract}




\section{Introduction}

Electronic cigarette (e-cigarette) use among various user groups has increased considerably over the past years ${ }^{1,2}$. One of the most important reasons for e-cigarettes' great popularity is the assortment of available e-liquid flavors ${ }^{3-6}$; for example, no less than 245 unique flavors were available in the Netherlands in $2017^{7}$. Flavors increase product attractiveness among all types of (potential) users, that is, among youth and adults ${ }^{8}$, and among current smokers, dual users, exclusive vapers, as well as never-users ${ }^{9}$. For smokers, switching to e-cigarettes may be beneficial, as e-cigarette use (i.e., vaping) is considered less harmful than regular cigarette smoking ${ }^{10-14}$. In line with this, the use and marketing of e-liquid flavors that are appealing to smokers may contribute to public health benefits. However, flavors may also stimulate vaping among non-users, in particular young people ${ }^{15-17}$. This is concerning, as e-cigarettes are not safe ${ }^{10,18,19}$. That is, chemicals in e-cigarette emissions (e.g., tobacco-specific nitrosamines, metals, aldehydes, and other flavorings) can be toxic and thus harmful to consumers' health 20-22. In addition, e-cigarettes may facilitate smoking initiation among never-smokers ${ }^{23}$. As a consequence, e-liquid flavors are considered an important target in tobacco control in order to decrease e-cigarette attractiveness and use and thereby exposure to potentially toxic emissions.

One way of regulating e-liquid flavors could be restriction of flavor categories that are particularly appealing to non-users or youth. For example, the US Food and Drug Administration (FDA) recently announced that e-cigarettes with a flavor other than tobacco will be removed from the market until they are evaluated under the FDA's new product review authority ${ }^{24}$. Such rules often rely on flavor-related marketing descriptions, which do not necessarily reflect human perception and may differ between e-liquid providers and therefore can be challenging to enforce. In addition, allowing or banning a specific e-liquid flavor may be complicated as e-liquids regularly have multiple flavor descriptors (e.g., tobacco with caramel and vanilla) 7. Similarly, using sensory analysis to enforce e-liquid flavor regulations may be challenging. Although based on human perception, analyzing sensory properties of all e-liquids in a particular country to determine whether they comply with current regulations is extremely time-consuming. However, sensory analysis could be used to determine attractiveness of particular e-liquid flavors or flavor categories among vulnerable user groups (e.g., non-smoking adolescents), and in that way inform policy makers on how to reduce e-liquid appeal. Another option would be to decrease e-liquid attractiveness or toxicity by restricting the addition of particular flavoring ingredients. For example, particular flavorings could be banned or their maximum concentration could be restricted. This may diminish e-cigarette use and thereby exposure to potentially toxic e-liquid constituents, and thus increase health benefits for non-users and youth.

Research on e-liquid flavors and flavoring compositions can support regulators in developing policy measures. Accordingly, various chemical-analytical studies evaluated e-liquid flavoring ingredients and emissions ${ }^{21,25-29}$. These studies typically focus on a list of a priori selected target flavorings for their analyses ${ }^{21,25-29}$. These target lists are usually selected based on previous studies, which creates a risk of selection bias by overlooking other or new e-liquid ingredients that may have toxic properties. Therefore, there is a clear need for a published overview of common flavorings and other ingredients in e-liquids. This information may be used as a starting point for future chemical analytical researchers in developing their target lists, may 
provide targets for future toxicological studies, and may provide foci for regulation of e-liquid flavorings.

We previously generated an overview of all e-liquid flavor descriptions reported to be marketed in the Netherlands in $2017^{7}$, by manually classifying almost 20000 e-liquids into 16 main flavor categories ${ }^{30}$. Classification was based on e-liquids' flavor-related information reported by manufacturers. In the European Union, manufacturers are also required to provide information about their e-liquids' chemical composition. Using this information, the current study firstly aims to identify the most commonly used e-liquid flavorings in general, and to determine potential flavorings that are specific to a single flavor category.

In our previous study, we used information from manufacturers such as brand names to manually classify e-liquids by flavor description ${ }^{7}$. However, 2586 e-liquids (15\% of the entire dataset) could not be classified as flavor-related information was unspecific, incomplete or even unavailable. For example, it was not possible to classify e-liquids with generic brand names that are unrelated to a flavor (e.g., "Spaceship", or "Purple Unicorn", hypothetically). Manufacturers additionally reported information about their e-liquids' chemical composition. Therefore, the second aim of this study is to determine whether this information can be used to predict e-liquids' flavor categories, using a machine learning algorithm. Such automatic classification of e-liquids by flavor would allow to easily create market overviews of e-liquid flavor descriptions worldwide in a time- and cost-effective manner, provided that information on e-liquid ingredients is available. 


\section{Methods}

\section{Data collection and preparation}

According to the European Tobacco Product Directive, tobacco and e-liquid manufacturers are required to provide information such as brand names, ingredients, and emissions of the products they have marketed in each Member State. A complete dataset of all e-cigarette products on the Dutch market was extracted from the European Common Entry Gate system (EU-CEG) ${ }^{31}$ on 30 November 2017. For this study, only e-liquids were included (i.e., no other products such as devices). Duplicate submissions and products with incomplete information were excluded, resulting in a dataset of 19266 products.

In a previous study, flavor-related information about each e-liquid was obtained from the EU-CEG system. According to a standardized approach, e-liquid flavors were classified into one of the following 16 main flavor categories: tobacco, menthol/mint, nuts, spices, coffeel tea, alcohol, other beverages, fruit-berries, fruit-citrus, fruit-tropical, fruit-other, dessert, candy, other sweets, other flavors, and unflavored ${ }^{7,30}$. E-liquids were considered "unclassifiable" if they were not found on the internet ( $\mathrm{n}=1680 ; 9 \%$ of total sample) or could not be searched for due to the EU-CEG information being too general (e.g., only referring to a brand or product range, $\mathrm{n}=$ $906 ; 5 \%$ of total sample) ${ }^{7}$.

\section{Data analyses}

As most e-liquids were reported as being marketed in a package unit of " 1 " and containing 10 $\mathrm{mL}$ of fluid, deviating submissions (more than 1 e-liquid per package or e-liquids with a volume other than $10 \mathrm{~mL}$ ) were excluded. This resulted in a final dataset of 16839 products. For these products, ingredient-related information was extracted from the EU-CEG system, and analyzed using R statistical software V.3.5.1. Ingredients reported by manufacturers as having the function "Flavour and/or Taste Enhancer" will be referred to as flavorings. Negative values for ingredient amounts (resulting from EU-CEG artefacts) were set at zero. For the overall dataset, as well as for individual flavor categories and the unclassifiable subset separately, the following values were determined: the number of products, the mean number of total ingredients, the mean number of flavorings per product, the mean total number of ingredients per product, and the mean quantities of all flavorings per product.

Ingredients present under multiple names in the EU-CEG system (e.g. ethanol, etanolo, etanol, ethyl alcohol, ethyl alkohol, ethyl-alcohol, alcool ethylique, and EtOH) were merged into one ingredient name. First, unique ingredient names were identified $(n=8352)$, including the number of products for which they were reported in the EU-CEG system. Next, starting with the most frequently reported ingredients names, we manually searched for other names that represented the same ingredient and thus could be grouped together. This was done using CAS registry numbers (i.e., assigned by the Chemical Abstracts Service), FEMA registry numbers (i.e., assigned by the Flavor Extract Manufacturers Association), trivial names, translations of ingredient names, and text cleanup (e.g., removing upper/lower case redundancy and spelling mistakes). This process was repeated until all ingredient names that were initially reported in more than 100 products (i.e., more than $0.6 \%$ of all products) were checked. This resulted in a final list of 219 unique ingredients. 
For further analysis, solvents (propylene glycol, glycerol, ethanol, water, and triacetin) and nicotine were excluded. For the remaining 213 flavorings, we identified the flavorings that were present in at least $10 \%$ of all products ( $\mathrm{n}=25$ flavorings), as well as the median quantity $(\mathrm{mg} / 10 \mathrm{~mL})$ in which they were added. This was also done for each individual flavor category and for the set of unclassifiable products ( $\mathrm{n}=94$ flavorings in total).

Next, quantitative information of the flavorings that were present in at least $10 \%$ of the products in any flavor category were used for machine learning prediction of an e-liquid's class (i.e., flavor category) using the random forest (RF) algorithm ${ }^{32}$ in the randomForest $\mathrm{R}$ package. First, the 14253 products that were assigned to one of the 16 flavor categories were used for RF classification. A fivefold cross-validation was used, for which the data were randomly split into five subsets containing approximately the same number of products and similar distributions of the flavor categories. Next, ingredient information about $80 \%(4 / 5$ subsets) of the products was used to train a model that predicted the class of the other $20 \%(1 / 5)$ of the products; this was done five times. Additional R settings selected included the number of trees (ntree $=2000$ ) and the option to return both the predicted class label and the probabilities for each class. Resulting data were used to evaluate the overall prediction accuracy. For this, we determined how many products were assigned to the correct class according to the RF model (i.e., the flavor category with the highest probability). In addition, we determined for how many incorrectly assigned products the correct class received the second highest probability according the RF model (including tied second place). To determine the chance-based prediction accuracy, we randomly reassigned each product to one of the categories and repeated the machine learning analysis. This resulted in an overall chance accuracy of $10.2 \%$. Finally, we trained a model using quantitative information about the complete set of 14253 products with an assigned flavor category to predict the class of the 2585 products defined as "unclassifiable" in our previous study 7

Because quantitative information is not always reported, the analyses were repeated using qualitative information about the ingredients only to provide a proof of principle that the method can also be used for qualitative data. 


\section{Results}

\section{Mean number of flavorings}

Over all 16839 e-liquids, the mean number of reported flavorings per e-liquid was $10 \pm 15$. Figure 4.1 shows the mean number of flavorings and other ingredients in total and for each of the separate flavor categories. The mean number of flavorings per flavor category (excluding unflavored) ranged from $3 \pm 8$ (for nuts) to $18 \pm 20$ (for dessert).

On average, $63 \%$ of the total number of ingredients within one e-liquid were flavorings. The mean number of flavorings as percentage of the total number of ingredients (excluding unflavored) was highest for e-liquids classified as candy (75\% were flavorings) and lowest for nuts (23\% were flavorings). The median concentration of total flavorings per e-liquid was 28.0 $\mathrm{mg} / 10 \mathrm{~mL}$.

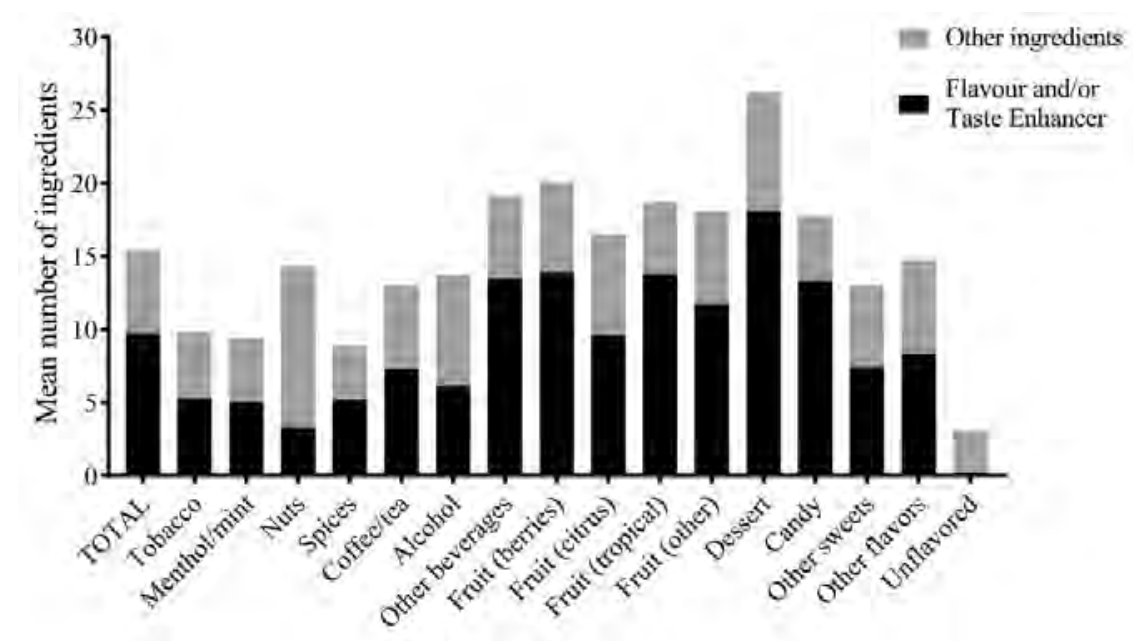

Figure 4.1: Mean number of ingredients indicated as having a "Flavour and/or Taste Enhancer" function (black) and ingredients with another function (grey) in total and for each of the separate flavor categories. Other functions of ingredients may include addictiveness enhancers, carriers, casings, fibres, humectants, solvents, processing aids, smoke odor modifiers, water-wetting agents, and viscosity modifiers ${ }^{33}$.

\section{Most frequently added flavorings and their quantities}

We identified 219 unique ingredients reported to be added to more than 100 e-liquids of the entire dataset. An overview of these ingredients, including their prevalence, is shown in Appendix Table A4.1. This overview covers $99.4 \%$ of all unique ingredients $(n=8352)$ reported. Ingredients other than flavoring ingredients were glycerol, nicotine, propylene glycol, water, ethanol, and triacetin. These compounds were present in respectively $94 \%, 88 \%, 86 \%, 45 \%, 23 \%$, and $15 \%$ of all e-liquids. 
Twenty-five flavoring ingredients were added to more than $10 \%$ of the overall sample of e-liquids (see Table 4.1). The most frequently used flavorings were vanillin (present in $35.2 \%$ of the total set), ethyl maltol $(32.0 \%)$ and ethyl butyrate $(28.4 \%)$. The highest median concentration was reported for menthol $(18.4 \mathrm{mg} / 10 \mathrm{~mL})$ and the lowest median concentration was reported for benzaldehyde $(0.3 \mathrm{mg} / 10 \mathrm{~mL})$.

The five flavorings that were most frequently used per separate flavor category are listed in Table 4.2. Appendix Table A4.2 shows an overview of all flavorings added to more than $10 \%$ of the e-liquids for each flavor category separately. Only two flavorings, ethyl maltol and vanillin, were added to more than $10 \%$ of the e-liquids of all flavor categories (except for unflavored). On the other hand, 29 flavorings were added to more than $10 \%$ of the e-liquids in a single category (excluding those specific to the other flavors category). These "category-specific" flavorings were: $\beta$-damascone (for tobacco); eucalyptol, menthone, and peppermint oil (for $\mathrm{menthol} / \mathrm{mint}$ ); 2,5-dimethylpyrazine, 2-3-hexanedione, 4,5-dimethyl-3-hydroxy-2,5-dihydrofuran-2-one, 5-methyl furfural, $\gamma$-dodecalactone, and triethyl citrate (for nuts); anethole and trans-anethole (for spices); ethyl heptanoate, ethyl nonanoate, isoamyl alcohol, and lactic acid (for alcohol); cinnamaldehyde (for other beverages); dimethyl sulfide and propionic acid (for fruit-berries); orange oil (for fruit-citrus); isobutyl acetate and trans-2-hexenal (for fruit-other); and 4-methyl5-thiazole ethanol, anisyl alcohol, benzyl benzoate, $\gamma$-hexalactone, methyl- $\alpha$-ionone, methylthio-methyl-pyrazine, and propenyl guaethol (for dessert). See Appendix Table A4.3 for flavor descriptions of these ingredients. 


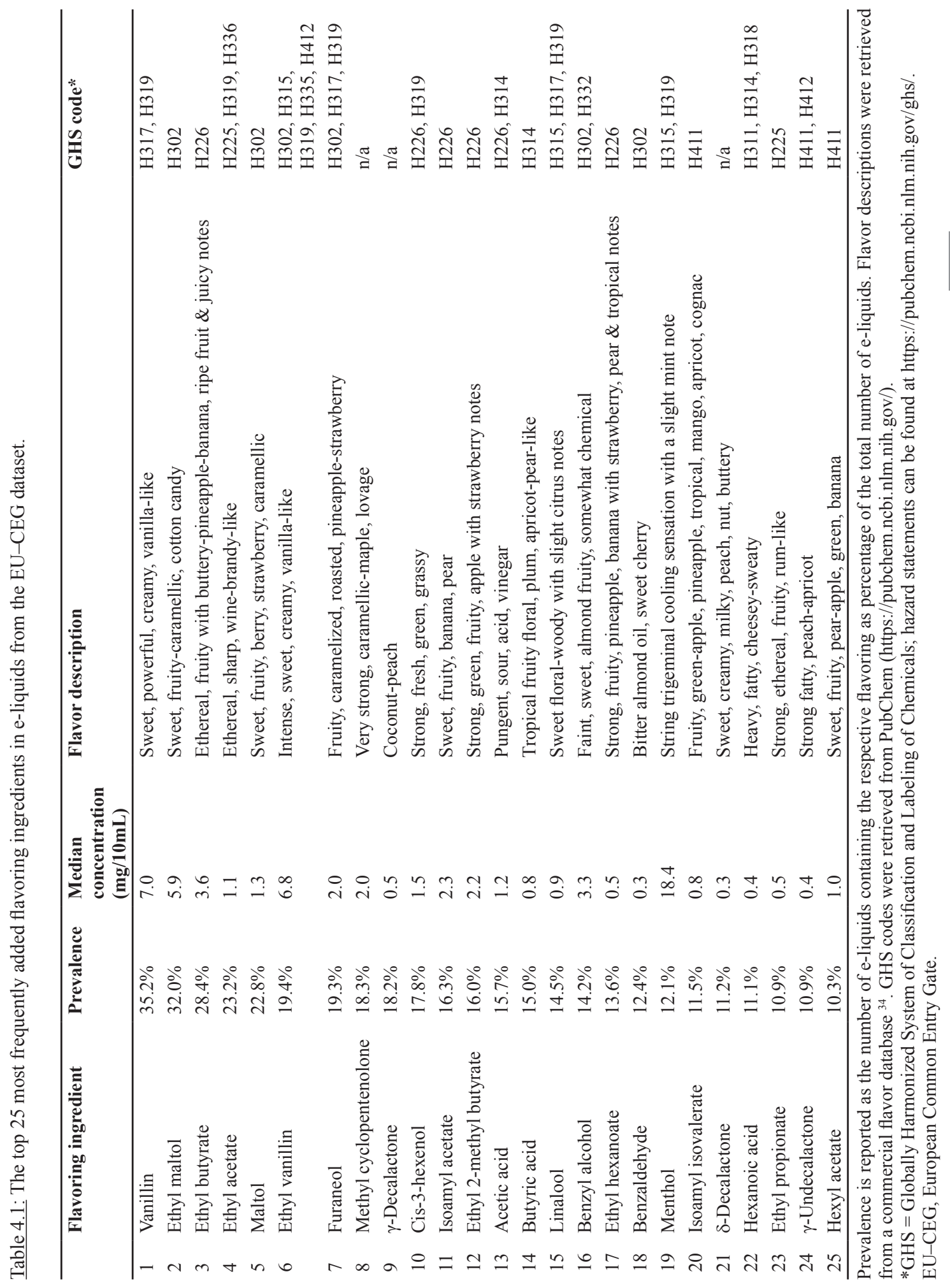


Table 4.2: Overview of the top 5 most frequently added flavorings for each individual flavor category ${ }^{30}$.

\begin{tabular}{|c|c|c|c|}
\hline Flavor category & Top 5 flavoring ingredients & Prevalence & Median concentration $(\mathrm{mg} / 10 \mathrm{~mL})$ \\
\hline \multirow[t]{5}{*}{ Tobacco } & Ethyl maltol & $31.2 \%$ & 6.8 \\
\hline & Methyl cyclopentenolone & $29.6 \%$ & 1.5 \\
\hline & Vanillin & $25.9 \%$ & 4.1 \\
\hline & 2,3,5-Trimethylpyrazine & $15.8 \%$ & 1.3 \\
\hline & Furaneol & $13.1 \%$ & 3.0 \\
\hline \multirow[t]{5}{*}{ Menthol/mint } & Menthol & $58.6 \%$ & 57.6 \\
\hline & Menthone & $16.6 \%$ & 22.7 \\
\hline & Ethyl maltol & $12.7 \%$ & 0.7 \\
\hline & Vanillin & $11.9 \%$ & 1.2 \\
\hline & Eucalyptol & $11.5 \%$ & 7.2 \\
\hline \multirow[t]{5}{*}{ Nuts } & Vanillin & $58.8 \%$ & 30.6 \\
\hline & Ethyl maltol & $47.5 \%$ & 24.5 \\
\hline & Ethyl vanillin & $35.0 \%$ & 32.0 \\
\hline & Acetoin & $27.5 \%$ & 24.0 \\
\hline & Maltol & $26.9 \%$ & 6.2 \\
\hline \multirow[t]{5}{*}{ Spices } & Menthol & $23.5 \%$ & 20.4 \\
\hline & Anethole & $22.8 \%$ & 60.0 \\
\hline & Ethyl maltol & $22.8 \%$ & 17.7 \\
\hline & Ethyl butyrate & $13.0 \%$ & 2.5 \\
\hline & Benzyl alcohol & $11.7 \%$ & 28.0 \\
\hline \multirow[t]{5}{*}{ Coffee/tea } & Vanillin & $36.0 \%$ & 5.7 \\
\hline & Methyl cyclopentenolone & $21.4 \%$ & 5.7 \\
\hline & Benzyl alcohol & $20.9 \%$ & 17.0 \\
\hline & Ethyl maltol & $15.3 \%$ & 3.3 \\
\hline & Ethyl vanillin & $15.0 \%$ & 2.5 \\
\hline \multirow[t]{5}{*}{ Alcohol } & Vanillin & $50.3 \%$ & 9.0 \\
\hline & Ethyl acetate & $25.7 \%$ & 7.9 \\
\hline & Ethyl butyrate & $23.1 \%$ & 6.0 \\
\hline & Ethyl propionate & $18.8 \%$ & 15.0 \\
\hline & Ethyl heptanoate & $18.5 \%$ & 2.2 \\
\hline \multirow[t]{5}{*}{ Other beverages } & Vanillin & $44.5 \%$ & 7.2 \\
\hline & Ethyl butyrate & $39.6 \%$ & 2.2 \\
\hline & Ethyl maltol & $36.6 \%$ & 3.2 \\
\hline & Ethyl acetate & $30.9 \%$ & 0.8 \\
\hline & Ethyl vanillin & $30.8 \%$ & 5.4 \\
\hline \multirow[t]{5}{*}{ Fruit-berries } & Ethyl butyrate & $52.4 \%$ & 6.2 \\
\hline & Cis-3-hexenol & $41.2 \%$ & 2.3 \\
\hline & Vanillin & $39.1 \%$ & 3.1 \\
\hline & Furaneol & $36.8 \%$ & 3.3 \\
\hline & Ethyl acetate & $36.0 \%$ & 1.4 \\
\hline
\end{tabular}




\begin{tabular}{|c|c|c|c|}
\hline \multirow[t]{5}{*}{ Fruit-citrus } & Ethyl maltol & $32.9 \%$ & 6.0 \\
\hline & Ethyl butyrate & $31.1 \%$ & 2.9 \\
\hline & Vanillin & $31.0 \%$ & 6.0 \\
\hline & Ethyl acetate & $26.3 \%$ & 0.7 \\
\hline & Linalool & $25.8 \%$ & 1.6 \\
\hline \multirow[t]{5}{*}{ Fruit-tropical } & Ethyl butyrate & $38.5 \%$ & 2.4 \\
\hline & Vanillin & $36.5 \%$ & 2.8 \\
\hline & Isoamyl acetate & $32.0 \%$ & 2.2 \\
\hline & Ethyl acetate & $31.2 \%$ & 1.1 \\
\hline & Ethyl maltol & $30.7 \%$ & 1.3 \\
\hline \multirow[t]{5}{*}{ Fruit-other } & Ethyl butyrate & $42.6 \%$ & 3.8 \\
\hline & Ethyl acetate & $37.7 \%$ & 3.1 \\
\hline & Isoamyl acetate & $35.9 \%$ & 3.6 \\
\hline & Vanillin & $32.0 \%$ & 2.8 \\
\hline & Maltol & $28.1 \%$ & 1.4 \\
\hline \multirow[t]{5}{*}{ Dessert } & Vanillin & $74.5 \%$ & 21.8 \\
\hline & Ethyl maltol & $64.8 \%$ & 9.0 \\
\hline & Ethyl vanillin & $63.0 \%$ & 13.8 \\
\hline & Maltol & $50.9 \%$ & 1.4 \\
\hline & Methyl cyclopentenolone & $49.2 \%$ & 1.2 \\
\hline \multirow[t]{5}{*}{ Candy } & Ethyl maltol & $39.4 \%$ & 0.5 \\
\hline & Isoamyl acetate & $37.4 \%$ & 2.3 \\
\hline & Ethyl butyrate & $37.2 \%$ & 1.1 \\
\hline & Vanillin & $35.2 \%$ & 1.1 \\
\hline & Ethyl acetate & $35.0 \%$ & 0.4 \\
\hline \multirow[t]{5}{*}{ Other sweets } & Vanillin & $61.4 \%$ & 30.0 \\
\hline & Ethyl maltol & $37.5 \%$ & 28.0 \\
\hline & Ethyl vanillin & $35.0 \%$ & 19.7 \\
\hline & Maltol & $26.0 \%$ & 1.3 \\
\hline & Piperonal & $24.4 \%$ & 3.2 \\
\hline \multirow[t]{5}{*}{ Other flavors } & Linalool & $30.5 \%$ & 3.0 \\
\hline & Ethyl butyrate & $25.2 \%$ & 6.0 \\
\hline & Ethyl acetate & $23.2 \%$ & 8.0 \\
\hline & Maltol & $23.2 \%$ & 4.1 \\
\hline & Ethyl 2-methyl butyrate & $22.5 \%$ & 5.9 \\
\hline Unflavored & NA & NA & NA \\
\hline
\end{tabular}

Prevalence is reported as the number of e-liquids containing the respective flavoring as percentage of the total number of e-liquids within that category. For a complete overview, see Appendix Table A4.1. See Appendix Table A4.3 for flavor descriptions of these ingredients. 


\section{Predictive value of flavoring composition}

Using information about ingredient quantities, 9982 of 14253 e-liquids were assigned to the correct flavor category. This means that the overall prediction accuracy of the RF algorithm was $70 \%$. For 3740 incorrectly assigned products ( $26 \%$ of total e-liquid sample), the correct class (i.e., the manually assigned class) received the second highest probability.

Using the RF model to predict the flavor category of the e-liquids that were defined as "unclassifiable" in our previous study ${ }^{7}$ resulted in: 56\% of these e-liquids being classified as tobacco; $10 \%$ as fruit-other; $9 \%$ as fruit-berries; $7 \%$ as menthol $/$ mint; $7 \%$ as dessert; 3\% as alcohol; $3 \%$ as other sweets; $2 \%$ as fruit-citrus; $2 \%$ as fruit-tropical; $1 \%$ as coffeeltea; and $1 \%$ as other beverages (see Appendix Table A4.4).

Finally, when only using information about the presence of ingredients rather than their amounts (a qualitative rather than quantitative approach), the RF algorithm had an overall prediction accuracy of $66 \%$. 


\section{Discussion}

This study provides an overview of frequently added ingredients, and their quantities, in all e-liquids available on the Dutch market in 2017. In total, 219 unique ingredients were identified, covering $99.4 \%$ of all ingredients reported to be used in our dataset of 16839 e-liquids. On average, $63 \%$ of the total number of ingredients per e-liquid were flavorings and the mean number of flavorings per e-liquid was 10. Common non-flavoring ingredients were glycerol, nicotine, propylene glycol, water, ethanol, and triacetin. Vanillin, also one of the mostly used additives in tobacco products ${ }^{35}$ for its sweet, creamy, vanilla-like flavor ${ }^{34}$, was the flavoring most frequently added to e-liquids. The overall median concentration was highest for menthol: a compound that is commonly added to tobacco products for its cooling and smoothing effects ${ }^{36}$. The highest mean numbers of flavorings were found for flavor categories that typically contain sweet e-liquids such as dessert, other beverages, fruit, and candy. As our results showed category-specific flavoring patterns, we were able to successfully predict an e-liquid's flavor category based on patterns of flavoring compositions ( $70 \%$ accuracy).

\section{Main applications}

Our study provides a comprehensive overview of flavorings added to e-liquids, including their quantities, thereby giving directions to other researchers for selection of target compounds in their future chemical-analytical and toxicological studies regarding e-liquid flavorings. For example, based on their research questions, researchers can use our data to select any number of most frequently used flavorings, or flavorings that are unique to particular flavor categories.

In addition, our study showed that using a machine-learning algorithm on a dataset on e-liquid flavoring compositions can provide a reliable estimation of marketed e-liquid flavors. The algorithm can be successfully applied using both ingredient quantities as well as qualitative information only. The resulting overview of marketed e-liquid flavors and their distribution across categories can be used for comparative analyses between regions or countries, and to keep track of market trends. Finally, regulators can combine our results with sensory data on flavor preferences from (potential) consumers to define regulatory targets for reducing e-liquid appeal and use among vulnerable user groups (e.g., non-smokers and youth).

\section{Considerations regarding the information source}

It should be noted that the information used in this study was provided by the industry, without the aim of sustaining research, and were not validated by an independent party. Therefore, while manufacturers were instructed to report ingredient quantities in weight per product unit (i.e., $\mathrm{mg} / 10 \mathrm{~mL})^{33}$, it is uncertain whether all ingredients were reported in the correct unit. Due to the large size of the dataset used in this study, potential data artefacts are expected to not have influenced overall results. Yet, as information was provided by the industry, results should be interpreted with appropriate caution. In addition, as the EU-CEG information used in this study was extracted at a single time point, results may not be generalizable over time. Therefore, regular follow-up analyses would be worthwhile to get more insight into the dynamics of the e-liquid flavor market. 


\section{Benefits and limitations of automatic classification}

The overall accuracy of predicting an e-liquid's flavor category based on patterns of flavoring compositions was $70 \%$ using ingredient quantities. This is almost 7 times higher than chance level (10.2\%), and was only slightly lower when only qualitative information was used (66\%). This shows that our approach provides a reliable estimation of the distribution of flavor categories on market or country level. It should be noted that the algorithm is not necessarily suitable for predicting flavor categories of individual products. Nevertheless, an important benefit of automatically classifying e-liquids over manual classification is that it significantly limits the time and workload needed to classify a large sample of products.

Other important advantages of classification based on ingredients are that it is insensitive to interpretation of flavor descriptions, and allows for classification of products with ambiguous brand names (e.g., "Spaceship", hypothetically) or lacking flavor-related marketing information. In our previous study, a set of 2586 e-liquids could not be classified in any flavor category, due to unavailable or unspecific flavor-related information. However, using the RF algorithm and ingredient information, we were able to classify these e-liquids in addition to the other 14253 products, thus provide a complete census of e-liquids on the Dutch market.

While the overall prediction accuracy was high, $30 \%$ of all e-liquids were misclassified. It is important to note that, in this study, an e-liquid was considered "misclassified" when the $\mathrm{RF}$ algorithm assigned it to a flavor category different from the one it was assigned to in the manual classification approach 7. The term "misclassified" is debatable: an e-liquid that was misclassified by the RF algorithm may actually better fit the category to which it was assigned by the algorithm than the category to which it was assigned by manual classification. For example, a hypothetical e-liquid marketed as "strawberry and mint" would be manually classified in the fruit-berries category, because strawberry was the first flavor description mentioned (see classification guidelines in $\operatorname{ref}^{30}$ ). However, this e-liquid may contain more menthol-related than strawberry-related flavoring ingredients, thus being potentially classified in the menthol/mint category by the RF algorithm. Whether the correct flavor category is fruit-berries or menthol/ mint is debatable, so this type of considerations increases difficulties regarding e-liquid flavor classification.

In this study, for most of the e-liquids that were misclassified (87.5\%), the correct class received the second highest probability. This shows that, if the algorithm assigned a product to the wrong class, the correct flavor category was usually second best. In these cases, the e-liquid flavor closely relates to multiple flavor categories. This can be caused by one e-liquid containing ingredients with flavor descriptions related to multiple categories, or ambiguous flavor-related marketing information that can be interpreted as relating to more than one flavor category. For example, an e-liquid marketed as "apple pie" (and thus classified in the main category dessert) may contain a mixture of ingredients that are separately described as having an apple, cinnamon, or pie flavor. Due to this combination of flavorings, this e-liquid may be as much likely to be classified in the fruit-other, spices, or dessert category. Other examples of such misclassifications in our dataset are: (1) an e-liquid with "peanut butter, vanilla \& banana" flavor, incorrectly assigned to other sweets while manually classified as nuts, and (2) an e-liquid with "lemonade" flavor, incorrectly assigned to fruit-citrus while manually classified as other beverages. The existence of products with such combined flavors makes both automatic and manual flavor 
classification challenging.

To improve classification results, classification of some products could be reconsidered, particularly of the products of which the probability of the assigned category is rather low, or of which probabilities of the assigned category and the second-best category differ not much. For example, this could be a product that is classified in the fruit-other category with a probability of $49 \%$ and received a probability of $46 \%$ for the dessert category (second-best). These e-liquids could be manually reclassified, for example, using flavor-related information from Web shops or using sensory analysis, provided that these e-liquids are still on the market at the time of data analysis. It should be noted that, although classifying e-liquids based on human expert judgement would be an accurate approach, training a panel to identify each e-liquid's flavor would be timeconsuming.

Relatively most misclassifications occurred in categories containing a low number of products (i.e., unflavored, nuts, spices), which may be due to the fact that they contained fewer examples for training the algorithm. Besides, a relatively high number of products from the other flavors category were incorrectly assigned to other categories. This seems inevitable as the e-liquids in this category do not have a certain common flavor, but instead have miscellaneous flavors that did not fit in any other category. Hence, correct classification for the other flavors category is more difficult, but also less relevant as this category is by definition a heterogeneous group.

Finally, it should be noted that our analyses were based on flavorings that were reported to be added to more than 100 e-liquids of the entire dataset (i.e., more than $0.6 \%$ of the 16839 products). Hence, a few seldom reported ingredients are not represented in our comprehensive overview of e-liquid ingredients. In addition, only e-liquids with a volume of $10 \mathrm{~mL}$ were included in this study. Therefore, results do not include cartridges and pre-filled e-cigarettes.

\section{Conclusions and recommendations}

We analyzed e-liquid ingredients and provided an overview of the flavoring ingredients most frequently reported to be added to e-liquids, and their quantities. Besides similarities between flavor categories, we identified flavorings that were specific to only one flavor category. Moreover, we successfully predicted e-liquids' flavor categories based on their flavoring composition with $70 \%$ accuracy. Automatically classifying e-liquids in this way allows to quickly generate an overview of marketed e-liquid flavor descriptions in a particular country. Thus, we recommended regulators to request information from manufacturers regarding the compositions of all marketed e-liquids to be able to perform similar analyses. This may help to support compliance and control of potential future regulations of e-liquid flavors. 


\section{What this paper adds}

What is already known on this subject: Flavoring ingredients increase e-cigarette attractiveness and use, particularly for young non-smokers, and thereby increase exposure to potentially toxic ingredients. Chemical analysis can be used to identify and quantify e-liquid flavoring ingredients. What important gaps in knowledge exist on this topic: A comprehensive overview of e-liquid ingredients is needed as a starting point for chemical-analytical and toxicological research, and for regulatory approaches aimed at reducing e-cigarette attractiveness. Instead of manual classification of e-liquids by flavor using marketing descriptions, an automatic approach is needed to create market overviews of e-liquid flavors worldwide in a time-efficient manner. What this paper adds: This study is the first to use information from manufacturers to create a comprehensive overview of e-liquid flavorings and their quantities, which may give direction to future research on e-liquid flavorings and may support flavoring-level regulation to decrease attractiveness of e-liquids. Our approach of automatically classifying e-liquids into flavor categories using their compositions was successful (70\% accuracy), and allows to quickly generate market overviews of e-liquid flavors that can be compared between countries. 


\section{Appendix}

Appendix Table A4.1: An overview of all 219 unique ingredients reported to be added to at least 100 e-liquids of the entire data set, including the percentage of e-liquids containing the ingredient, presented in alphabetical order.

\begin{tabular}{|c|c|}
\hline E-liquid ingredient & Prevalence \\
\hline$\alpha$-Damascenone & $0.02 \%$ \\
\hline$\alpha$-Damascone & $1.84 \%$ \\
\hline$\alpha$-Ionone & $5.55 \%$ \\
\hline$\alpha$-Methylbenzyl acetate & $1.80 \%$ \\
\hline$\alpha$-Pinene & $2.14 \%$ \\
\hline$\alpha$-Terpineol & $5.51 \%$ \\
\hline$\beta$-Caryophyllene & $1.81 \%$ \\
\hline$\beta$-Damascenone & $4.42 \%$ \\
\hline$\beta$-Damascone & $5.53 \%$ \\
\hline$\beta$-Ionone & $7.54 \%$ \\
\hline$\beta$-Pinene & $2.14 \%$ \\
\hline -Decalactone & $18.16 \%$ \\
\hline$\gamma$-Dodecalactone & $2.99 \%$ \\
\hline v-Hexalactone & $5.10 \%$ \\
\hline$\gamma$-Nonalactone & $9.53 \%$ \\
\hline v-Octalactone & $7.27 \%$ \\
\hline -Terpinene & $0.82 \%$ \\
\hline$\gamma$-Undecalactone & $10.86 \%$ \\
\hline$\gamma$-Valerolactone & $0.74 \%$ \\
\hline$\delta$-Decalactone & $11.21 \%$ \\
\hline$\delta$-Dodecalactone & $8.65 \%$ \\
\hline 2,3-Pentanedione & $3.09 \%$ \\
\hline 1-Pentanol & $1.05 \%$ \\
\hline 1,4-Dimethoxybenzene & $1.91 \%$ \\
\hline 2-3-Hexanedione & $1.07 \%$ \\
\hline 2-Acetylfuran & $1.18 \%$ \\
\hline 2-Acetylpyridine & $1.96 \%$ \\
\hline 2-Acetylpyrazine & $6.00 \%$ \\
\hline 2-Ethyl-3-methyl pyrazine & $0.79 \%$ \\
\hline 2-Isopropyl-4-methyl thiazole & $0.47 \%$ \\
\hline $\begin{array}{l}\text { 2-Isopropyl-N,2,3- } \\
\text { trimethylbutyramide }\end{array}$ & $1.28 \%$ \\
\hline 2-Methoxy-3-methyl pyrazine & $0.47 \%$ \\
\hline 2-Methyl butyric acid & $9.77 \%$ \\
\hline
\end{tabular}

[Continued from page 97]

\begin{tabular}{lr}
\hline E-liquid ingredient & Prevalence \\
\hline Ethyl butyrate & $28.37 \%$ \\
Ethyl cinnamate & $1.50 \%$
\end{tabular}

Ethyl decanoate $\quad 1.63 \%$

Ethyl dodecanoate $\quad 0.84 \%$

Ethyl heptanoate $\quad 2.00 \%$

Ethyl hexanoate $\quad 13.56 \%$

Ethyl isovalerate $\quad 9.51 \%$

Ethyl lactate $\quad 3.60 \%$

Ethyl maltol $\quad 32.01 \%$

Ethyl menthane carboxamide $\quad 1.51 \%$

Ethyl methyl phenylglycidate $\quad 2.41 \%$

Ethyl nonanoate $\quad 1.50 \%$

Ethyl octanoate $\quad 2.26 \%$

Ethyl propionate $\quad 10.94 \%$

Ethyl vanillin $\quad 19.38 \%$

Ethyl vanillin propylene glycol $\quad 0.32 \%$

acetal

Eucalyptol $\quad 3.00 \%$

Eugenol $\quad 6.01 \%$

Fenugreek $\quad 1.62 \%$

Furaneol $\quad 19.31 \%$

Furfural 2.98\%

Furfuryl alcohol 2.32\%

Geranial $\quad 0.80 \%$

Geraniol $\quad 4.06 \%$

Geranyl acetate $\quad 3.51 \%$

Ginger oil $\quad 0.14 \%$

Glycerol* $\quad 94.14 \%$

Glyceryl 1-acetate $\quad 0.91 \%$

Guaiacol $\quad 6.05 \%$

Hexanal $\quad 3.63 \%$

Hexanoic acid $\quad 11.10 \%$

Hexyl acetate $\quad 10.35 \%$

Hexyl butyrate $\quad 1.71 \%$ 


\begin{tabular}{|c|c|c|c|}
\hline 2-Methylbutyl acetate & $2.23 \%$ & Ionone (mixed isomers) & $0.99 \%$ \\
\hline 2-Phenylethanol & $4.20 \%$ & Isoamyl acetate & $16.33 \%$ \\
\hline 2-Phenylethyl acetate & $0.57 \%$ & Isoamyl alcohol & $4.49 \%$ \\
\hline 2-Propanol & $1.43 \%$ & Isoamyl butyrate & $6.83 \%$ \\
\hline 2,3-Dimethylpyrazine & $2.08 \%$ & Isoamyl isovalerate & $11.46 \%$ \\
\hline 2,3,5-Trimethylpyrazine & $5.67 \%$ & Isoamyl phenyl acetate & $1.49 \%$ \\
\hline 2,3,5,6-Tetramethylpyrazine & $2.90 \%$ & Isobutyl acetate & $4.88 \%$ \\
\hline 2,5-Dimethylpyrazine & $1.54 \%$ & Isobutyl alcohol & $1.41 \%$ \\
\hline 2,6-Dimethyl-5-heptenal & $2.15 \%$ & Isobutyl butyrate & $0.69 \%$ \\
\hline 4-(4-Hydroxyphenyl)-2-butanone & $9.43 \%$ & Isobutyric acid & $0.50 \%$ \\
\hline 4-(4-Methoxyphenyl)butan-2-one & $1.06 \%$ & Isovaleraldehyde & $1.78 \%$ \\
\hline 4-Methyl-5-thiazole ethanol & $5.53 \%$ & Jasmine absolute & $0.89 \%$ \\
\hline 4-Terpinenol & $0.37 \%$ & L-Carvone & $0.80 \%$ \\
\hline 4,5-Dimethyl-3-hydroxy-2,5- & $1.38 \%$ & Lactic acid & $3.42 \%$ \\
\hline dihydro-furan-2-one & & Lemon oil & $6.35 \%$ \\
\hline 5-Methyl-2-phenyl-2-hexenal & $1.10 \%$ & Levulinic acid & $0.81 \%$ \\
\hline 5-Methyl furfural & $2.73 \%$ & Lime oil & $3.98 \%$ \\
\hline 6-Methyl-5-hepten-2-one & $0.80 \%$ & Limonene & $7.90 \%$ \\
\hline 6-Methyl coumarin & $0.60 \%$ & Linalool & $14.53 \%$ \\
\hline Acetal & $1.08 \%$ & Linalyl acetate & $3.41 \%$ \\
\hline Acetaldehyde & $2.02 \%$ & Maltol & $22.76 \%$ \\
\hline Acetic acid & $15.67 \%$ & Mandarin oil & $0.50 \%$ \\
\hline Acetoin & $7.47 \%$ & Menthol & $12.10 \%$ \\
\hline Allyl hexanoate & $5.54 \%$ & Menthone & $2.92 \%$ \\
\hline Amyl acetate & $1.88 \%$ & Menthyl acetate & $2.32 \%$ \\
\hline Amyl butyrate & $1.10 \%$ & Methyl- $\alpha$-ionone & $2.62 \%$ \\
\hline Anethole & $2.30 \%$ & Methyl anthranilate & $3.74 \%$ \\
\hline Anisaldehyde & $9.01 \%$ & Methyl cinnamate & $9.37 \%$ \\
\hline Anise oil & $0.97 \%$ & Methyl cyclopentenolone & $18.32 \%$ \\
\hline Anisyl acetate & $0.83 \%$ & Methyl dihydrojasmonate & $0.81 \%$ \\
\hline Anisyl alcohol & $7.04 \%$ & Methyl salicylate & $1.19 \%$ \\
\hline Benzaldehyde & $12.43 \%$ & Methyl thiobutyrate & $1.47 \%$ \\
\hline Benzaldehyde propylene glycol & $0.74 \%$ & Methyl trans-cinnamate & $0.77 \%$ \\
\hline acetal & & Methyl-thio-methylpyrazine & $2.45 \%$ \\
\hline Benzyl acetate & $9.20 \%$ & Myrcene & $1.85 \%$ \\
\hline Benzyl alcohol & $14.19 \%$ & n-Butanol & $1.25 \%$ \\
\hline Benzyl benzoate & $3.10 \%$ & n-Hexanol & $3.58 \%$ \\
\hline Benzyl butyrate & $1.41 \%$ & n-Octanal & $1.10 \%$ \\
\hline Blood orange oil & $1.48 \%$ & n-Propanol & $0.51 \%$ \\
\hline Bucchu leaf oil & $1.28 \%$ & Neral & $0.75 \%$ \\
\hline Butyl acetate & $2.33 \%$ & Nerol & $1.24 \%$ \\
\hline
\end{tabular}




\begin{tabular}{|c|c|c|c|}
\hline Butyl butyrate & $2.82 \%$ & Neryl acetate & $1.59 \%$ \\
\hline Butyl butyryl lactate & $3.95 \%$ & Nicotine* & $88.41 \%$ \\
\hline Butyric acid & $14.99 \%$ & Octanoic acid & $4.39 \%$ \\
\hline Capsicum oleoresin & $0.48 \%$ & Octanol & $0.74 \%$ \\
\hline Caramel & $2.38 \%$ & Oleic acid & $1.09 \%$ \\
\hline Carob & $1.47 \%$ & Orange oil & $3.72 \%$ \\
\hline Carvone & $1.45 \%$ & p-Cymene & $0.96 \%$ \\
\hline Cassia oil & $1.25 \%$ & Peppermint oil & $1.78 \%$ \\
\hline Cedrol & $2.08 \%$ & Peru balsam & $1.16 \%$ \\
\hline Cinnamaldehyde & $3.78 \%$ & Piperonal & $9.56 \%$ \\
\hline Cinnamon oil & $0.99 \%$ & Potassium sorbate & $1.12 \%$ \\
\hline Cinnamyl alcohol & $0.82 \%$ & Propenyl guaethol & $2.42 \%$ \\
\hline cis-3-Hexenol & $17.77 \%$ & Propionic acid & $4.27 \%$ \\
\hline cis-3-Hexenyl acetate & $9.18 \%$ & Propyl acetate & $0.33 \%$ \\
\hline cis-3-Hexenyl butyrate & $3.35 \%$ & Propylene glycol* & $85.78 \%$ \\
\hline cis-3-Hexenyl isovalerate & $0.78 \%$ & Sodium benzoate & $1.16 \%$ \\
\hline cis-6-Nonen-1-ol & $0.93 \%$ & Sodium citrate & $1.16 \%$ \\
\hline Citral & $5.62 \%$ & Spearmint oil & $1.07 \%$ \\
\hline Citric acid & $3.10 \%$ & Strawberry extract & $1.59 \%$ \\
\hline Citronellol & $1.88 \%$ & Sucralose & $8.25 \%$ \\
\hline Citronellyl acetate & $1.50 \%$ & Sugar & $1.26 \%$ \\
\hline Cocoa extract & $1.54 \%$ & Tabanone & $0.53 \%$ \\
\hline Coffee extract & $0.34 \%$ & Terpinolene & $0.84 \%$ \\
\hline Cornmint oil & $1.26 \%$ & Thio menthone & $1.62 \%$ \\
\hline D-Carvone & $0.35 \%$ & trans-2-Hexenal & $3.85 \%$ \\
\hline Decanal & $1.25 \%$ & trans-2-Hexenoic acid & $1.31 \%$ \\
\hline Decanoic acid & $1.94 \%$ & trans-2-Hexenol & $1.82 \%$ \\
\hline Dihydrocoumarin & $5.89 \%$ & trans-2-Hexenylacetaat & $0.94 \%$ \\
\hline Dimethyl anthranilate & $1.32 \%$ & trans-Anethole & $2.19 \%$ \\
\hline Dimethyl sulfide & $5.02 \%$ & Triacetin* & $14.44 \%$ \\
\hline Dodecane & $0.74 \%$ & Triethyl citrate & $4.10 \%$ \\
\hline Ethanol* & $23.12 \%$ & Vanilla extract & $0.79 \%$ \\
\hline Ethyl-3-hydroxy butyrate & $0.48 \%$ & Vanillin & $35.17 \%$ \\
\hline Ethyl 2-methyl butyrate & $15.99 \%$ & Vanillin propylene glycol acetal & $1.33 \%$ \\
\hline Ethyl 2-phenyl acetate & $1.41 \%$ & Veratraldehyde & $1.70 \%$ \\
\hline Ethyl acetate & $23.23 \%$ & Water* & $44.96 \%$ \\
\hline
\end{tabular}

\section{[Continued]}

Prevalence is reported as the number of e-liquids containing the respective flavoring as percentage of the total number of e-liquids. This overview covers $99.4 \%$ of all ingredients reported.

* Non-flavoring ingredients $(\mathrm{n}=6)$, marked in light grey. 
Appendix Table A4.2: An overview of all flavorings added to more than $10 \%$ of the e-liquids within a category, for each flavor category separately.

\begin{tabular}{|c|c|c|c|c|c|}
\hline Flavoring ingredient & Prevalence & $\begin{array}{r}\text { Median } \\
\text { concen- } \\
\text { tration } \\
(\mathrm{mg} / 10 \mathrm{~mL})\end{array}$ & Flavoring ingredient & Prevalence & $\begin{array}{r}\text { Median } \\
\text { concen- } \\
\text { tration } \\
(\mathrm{mg} / 10 \mathrm{~mL}) \\
\end{array}$ \\
\hline \multicolumn{3}{|c|}{ Category: Tobacco ( $n=8$ flavorings) } & Isoamyl acetate & $32.0 \%$ & 2.25 \\
\hline Ethyl maltol & $31.2 \%$ & 6.78 & Ethyl acetate & $31.2 \%$ & 1.09 \\
\hline Methyl cyclopentenolone & $29.6 \%$ & 1.50 & Ethyl maltol & $30.7 \%$ & 1.31 \\
\hline Vanillin & $25.9 \%$ & 4.11 & cis-3-Hexenol & $29.1 \%$ & 1.00 \\
\hline 2,3,5-Trimethylpyrazine & $15.8 \%$ & 1.32 & Ethyl hexanoate & $27.2 \%$ & 0.72 \\
\hline Furaneol & $13.1 \%$ & 2.97 & Furaneol & $26.2 \%$ & 0.72 \\
\hline$\beta$-Damascone & $12.6 \%$ & 1.10 & Maltol & $24.9 \%$ & 1.04 \\
\hline 2-Acetylpyrazine & $12.3 \%$ & 1.32 & $\gamma$-Decalactone & $23.8 \%$ & 0.49 \\
\hline Benzyl alcohol & $10.3 \%$ & 6.00 & Allyl hexanoate & $22.8 \%$ & 1.11 \\
\hline \multicolumn{3}{|c|}{ Category: Menthol/mint ( $n=6$ flavorings) } & Acetic acid & $21.8 \%$ & 1.20 \\
\hline Menthol & $58.6 \%$ & 57.60 & Ethyl 2-methyl butyrate & $19.7 \%$ & 1.47 \\
\hline Menthone & $16.6 \%$ & 22.68 & Ethyl vanillin & $19.6 \%$ & 1.78 \\
\hline Ethyl maltol & $12.7 \%$ & 0.74 & $\gamma$-Undecalactone & $19.2 \%$ & 0.33 \\
\hline Vanillin & $11.9 \%$ & 1.24 & Isoamyl isovalerate & $19.2 \%$ & 1.10 \\
\hline Eucalyptol & $11.5 \%$ & 7.15 & Butyric acid & $18.7 \%$ & 1.02 \\
\hline Peppermint oil & $11.4 \%$ & 9.87 & Benzyl alcohol & $18.4 \%$ & 2.73 \\
\hline \multicolumn{3}{|c|}{ Category: Nuts ( $n=32$ flavorings) } & $\gamma$-Nonalactone & $18.4 \%$ & 0.78 \\
\hline Vanillin & $58.8 \%$ & 30.63 & Benzaldehyde & $17.6 \%$ & 0.20 \\
\hline Ethyl maltol & $47.5 \%$ & 24.50 & Isoamyl butyrate & $15.8 \%$ & 1.54 \\
\hline Ethyl vanillin & $35.0 \%$ & 32.00 & Benzyl acetate & $15.4 \%$ & 0.24 \\
\hline Acetoin & $27.5 \%$ & 24.00 & Methyl cyclopentenolone & $15.4 \%$ & 0.33 \\
\hline Maltol & $26.9 \%$ & 6.17 & Limonene & $15.3 \%$ & 2.99 \\
\hline 2,3-Pentanedione & $23.8 \%$ & 24.00 & Hexanoic acid & $15.2 \%$ & 0.40 \\
\hline Methyl cyclopentenolone & $23.8 \%$ & 24.00 & Ethyl propionate & $15.0 \%$ & 0.37 \\
\hline Butyric acid & $22.5 \%$ & 24.00 & $\gamma$-Octalactone & $14.7 \%$ & 0.42 \\
\hline$\delta$-Decalactone & $22.5 \%$ & 6.43 & Linalool & $14.7 \%$ & 0.40 \\
\hline$\gamma$-Decalactone & $22.5 \%$ & 1.63 & Ethyl isovalerate & $14.4 \%$ & 0.25 \\
\hline Ethyl acetate & $21.9 \%$ & 24.00 & 2-Methyl butyric acid & $13.4 \%$ & 1.40 \\
\hline Ethyl butyrate & $21.9 \%$ & 24.00 & Lemon oil & $13.4 \%$ & 0.25 \\
\hline Acetic acid & $21.3 \%$ & 24.00 & Cis-3-hexenyl acetate & $12.7 \%$ & 1.00 \\
\hline Furaneol & $20.0 \%$ & 28.56 & Hexyl acetate & $12.7 \%$ & 0.83 \\
\hline Anisaldehyde & $19.4 \%$ & 24.00 & Methyl cinnamate & $12.2 \%$ & 0.20 \\
\hline Guaiacol & $19.4 \%$ & 24.00 & Sucralose & $11.8 \%$ & 8.98 \\
\hline$\delta$-Dodecalactone & $17.5 \%$ & 1.63 & $\delta$-Decalactone & $11.4 \%$ & 0.24 \\
\hline 2,3,5-Trimethylpyrazine & $16.9 \%$ & 1.10 & Eugenol & $11.2 \%$ & 2.63 \\
\hline
\end{tabular}




\begin{tabular}{|c|c|c|}
\hline Ethyl propionate & $16.9 \%$ & 24.00 \\
\hline 2-Acetylpyrazine & $15.6 \%$ & 10.48 \\
\hline Butyl butyryl lactate & $15.6 \%$ & 50.91 \\
\hline$\gamma$-Octalactone & $15.0 \%$ & 35.14 \\
\hline 2,5-Dimethylpyrazine & $13.8 \%$ & 0.56 \\
\hline 4,5-Dimethyl- & $11.9 \%$ & 3.26 \\
\hline \multicolumn{3}{|l|}{ 3-hydroxy-2,5- } \\
\hline \multicolumn{3}{|l|}{ dihydrofuran-2-one } \\
\hline 5-Methyl furfural & $11.9 \%$ & 4.63 \\
\hline Benzyl alcohol & $11.9 \%$ & 8.00 \\
\hline Ethyl lactate & $11.9 \%$ & 46.28 \\
\hline$\gamma$-Dodecalactone & $11.9 \%$ & 1.63 \\
\hline Triethyl citrate & $11.9 \%$ & 46.28 \\
\hline Piperonal & $10.6 \%$ & 9.00 \\
\hline Veratraldehyde & $10.6 \%$ & 2.17 \\
\hline 2-3-Hexanedione & $10.0 \%$ & 1.63 \\
\hline \multicolumn{3}{|c|}{ Category: Spices ( $n=9$ flavorings) } \\
\hline Menthol & $23.5 \%$ & 20.42 \\
\hline Anethole & $22.8 \%$ & 60.00 \\
\hline Ethyl maltol & $22.8 \%$ & 17.72 \\
\hline Ethyl butyrate & $13.0 \%$ & 2.49 \\
\hline Benzyl alcohol & $11.7 \%$ & 28.00 \\
\hline Ethyl vanillin & $11.7 \%$ & 27.37 \\
\hline trans-Anethole & $11.1 \%$ & 105.00 \\
\hline Vanillin & $11.1 \%$ & 30.60 \\
\hline Anisaldehyde & $10.5 \%$ & 0.36 \\
\hline \multicolumn{3}{|c|}{ Category: Coffee/tea ( $n=13$ flavorings) } \\
\hline Vanillin & $36.0 \%$ & 5.68 \\
\hline Methyl cyclopentenolone & $21.4 \%$ & 5.70 \\
\hline Benzyl alcohol & $20.9 \%$ & 17.00 \\
\hline Ethyl maltol & $15.3 \%$ & 3.25 \\
\hline Ethyl vanillin & $15.0 \%$ & 2.47 \\
\hline Furaneol & $15.0 \%$ & 2.35 \\
\hline Maltol & $15.0 \%$ & 3.00 \\
\hline$\delta$-Decalactone & $14.3 \%$ & 0.60 \\
\hline Acetic acid & $13.3 \%$ & 0.66 \\
\hline$\delta$-Dodecalactone & $11.6 \%$ & 0.55 \\
\hline Ethyl acetate & $11.6 \%$ & 0.47 \\
\hline Dihydrocoumarin & $11.1 \%$ & 0.97 \\
\hline Ethyl butyrate & $11.1 \%$ & 1.28 \\
\hline Category: Alcohol $(n=22$ & prings) & \\
\hline
\end{tabular}

\begin{tabular}{|c|c|c|}
\hline Piperonal & $11.1 \%$ & 0.44 \\
\hline Acetoin & $10.5 \%$ & 1.00 \\
\hline \multicolumn{3}{|c|}{ Category: Fruit-other ( $n=35$ flavorings) } \\
\hline Ethyl butyrate & $42.6 \%$ & 3.78 \\
\hline Ethyl acetate & $37.7 \%$ & 3.08 \\
\hline Isoamyl acetate & $35.9 \%$ & 3.57 \\
\hline Vanillin & $32.0 \%$ & 2.81 \\
\hline Maltol & $28.1 \%$ & 1.41 \\
\hline Ethyl maltol & $28.0 \%$ & 0.95 \\
\hline Ethyl 2-methyl butyrate & $25.8 \%$ & 1.49 \\
\hline Hexyl acetate & $25.8 \%$ & 1.16 \\
\hline cis-3-Hexenol & $25.7 \%$ & 1.17 \\
\hline Benzaldehyde & $24.1 \%$ & 1.45 \\
\hline$\gamma$-Decalactone & $22.4 \%$ & 0.29 \\
\hline Acetic acid & $20.5 \%$ & 1.21 \\
\hline Linalool & $19.9 \%$ & 0.88 \\
\hline$\gamma$-Undecalactone & $19.8 \%$ & 0.77 \\
\hline Furaneol & $18.6 \%$ & 1.00 \\
\hline Ethyl hexanoate & $17.9 \%$ & 0.24 \\
\hline cis-3-Hexenyl acetate & $17.7 \%$ & 0.51 \\
\hline Benzyl alcohol & $15.3 \%$ & 1.40 \\
\hline Benzyl acetate & $14.6 \%$ & 1.00 \\
\hline Ethyl vanillin & $14.3 \%$ & 1.88 \\
\hline Ethyl isovalerate & $13.9 \%$ & 1.20 \\
\hline Isobutyl acetate & $13.7 \%$ & 1.76 \\
\hline Butyric acid & $13.2 \%$ & 0.32 \\
\hline trans-2-Hexenal & $12.9 \%$ & 1.93 \\
\hline 2-Methyl butyric acid & $12.0 \%$ & 1.34 \\
\hline Ethyl propionate & $11.9 \%$ & 0.33 \\
\hline 4-(4-Hydroxyphenyl)- & $11.7 \%$ & 1.43 \\
\hline \multicolumn{3}{|l|}{ 2-butanone } \\
\hline Isoamyl isovalerate & $11.7 \%$ & 0.77 \\
\hline$\delta$-Decalactone & $11.0 \%$ & 0.20 \\
\hline Methyl cyclopentenolone & $10.7 \%$ & 0.50 \\
\hline$\beta$-Ionone & $10.5 \%$ & 0.13 \\
\hline Limonene & $10.5 \%$ & 1.77 \\
\hline Anisaldehyde & $10.3 \%$ & 0.92 \\
\hline Menthol & $10.3 \%$ & 16.59 \\
\hline Sucralose & $10.2 \%$ & 10.80 \\
\hline \multicolumn{3}{|c|}{ Category: Dessert ( $n=47$ flavorings) } \\
\hline Vanillin & $74.5 \%$ & 21.80 \\
\hline
\end{tabular}




\begin{tabular}{|c|c|c|c|c|c|}
\hline & & & & & \\
\hline Vanillin & $50.3 \%$ & 9.00 & Ethyl maltol & $64.8 \%$ & 9.02 \\
\hline Ethyl acetate & $25.7 \%$ & 7.92 & Ethyl vanillin & $63.0 \%$ & 13.76 \\
\hline Ethyl butyrate & $23.1 \%$ & 5.98 & Maltol & $50.9 \%$ & 1.37 \\
\hline Ethyl propionate & $18.8 \%$ & 15.00 & Methyl cyclopentenolone & $49.2 \%$ & 1.20 \\
\hline Ethyl heptanoate & $18.5 \%$ & 2.23 & Butyric acid & $45.7 \%$ & 1.00 \\
\hline Ethyl maltol & $17.6 \%$ & 10.85 & Ethyl butyrate & $44.4 \%$ & 1.40 \\
\hline Benzaldehyde & $16.5 \%$ & 6.00 & $\gamma$-Decalactone & $40.6 \%$ & 0.24 \\
\hline$\gamma$-Nonalactone & $15.6 \%$ & 5.62 & $\delta$-Decalactone & $39.5 \%$ & 0.36 \\
\hline Ethyl hexanoate & $15.3 \%$ & 0.90 & Ethyl acetate & $38.7 \%$ & 0.39 \\
\hline Acetic acid & $15.0 \%$ & 1.58 & Furaneol & $34.2 \%$ & 2.10 \\
\hline Maltol & $14.2 \%$ & 4.90 & $\gamma$-Nonalactone & $34.1 \%$ & 0.96 \\
\hline Allyl hexanoate & $13.9 \%$ & 2.33 & Isoamyl isovalerate & $33.5 \%$ & 0.51 \\
\hline Piperonal & $13.0 \%$ & 1.14 & Piperonal & $33.1 \%$ & 0.36 \\
\hline$\delta$-Decalactone & $12.7 \%$ & 0.23 & Anisaldehyde & $31.8 \%$ & 0.20 \\
\hline Methyl cyclopentenolone & $12.1 \%$ & 10.82 & 4-Methyl-5-thiazole & $30.2 \%$ & 0.35 \\
\hline Isoamyl alcohol & $11.8 \%$ & 0.61 & Ethanol & & \\
\hline Isoamyl acetate & $11.3 \%$ & 1.00 & Guaiacol & $30.0 \%$ & 0.24 \\
\hline Lactic acid & $11.3 \%$ & 15.00 & Ethyl propionate & $29.9 \%$ & 0.98 \\
\hline Ethyl vanillin & $11.0 \%$ & 9.41 & Anisyl alcohol & $29.4 \%$ & 0.72 \\
\hline Ethyl nonanoate & $10.4 \%$ & 1.00 & $\delta$-Dodecalactone & $29.0 \%$ & 0.29 \\
\hline Furaneol & $10.4 \%$ & 7.96 & Benzyl alcohol & $28.8 \%$ & 4.01 \\
\hline Menthol & $10.1 \%$ & 11.75 & Hexanoic acid & $27.4 \%$ & 1.14 \\
\hline \multicolumn{3}{|c|}{ Category: Other beverages ( $n=44$ flavorings) } & $\gamma$-Octalactone & $27.0 \%$ & 0.54 \\
\hline Vanillin & $44.5 \%$ & 7.19 & cis-3-Hexenol & $25.0 \%$ & 1.00 \\
\hline Ethyl butyrate & $39.6 \%$ & 2.20 & $\gamma$-Hexalactone & $24.8 \%$ & 0.54 \\
\hline Ethyl maltol & $36.6 \%$ & 3.23 & Acetoin & $24.0 \%$ & 1.36 \\
\hline Ethyl acetate & $30.9 \%$ & 0.75 & Ethyl 2-methyl butyrate & $22.2 \%$ & 1.51 \\
\hline Ethyl vanillin & $30.8 \%$ & 5.39 & Sucralose & $21.8 \%$ & 8.68 \\
\hline Maltol & $28.2 \%$ & 0.90 & Acetic acid & $21.3 \%$ & 0.44 \\
\hline Isoamyl acetate & $26.4 \%$ & 1.75 & Dihydrocoumarin & $21.3 \%$ & 1.00 \\
\hline Ethyl 2-methyl butyrate & $25.5 \%$ & 0.25 & 2-Acetylpyrazine & $20.5 \%$ & 1.26 \\
\hline Furaneol & $25.0 \%$ & 0.66 & Benzaldehyde & $20.0 \%$ & 0.10 \\
\hline Ethyl hexanoate & $23.7 \%$ & 0.37 & Ethyl hexanoate & $17.6 \%$ & 0.62 \\
\hline Linalool & $23.5 \%$ & 0.31 & Linalool & $17.2 \%$ & 0.79 \\
\hline Methyl cinnamate & $22.0 \%$ & 0.18 & 2-Methyl butyric acid & $16.4 \%$ & 1.18 \\
\hline cis-3-Hexenol & $21.9 \%$ & 1.00 & Methyl cinnamate & $14.7 \%$ & 1.00 \\
\hline$\gamma$-Decalactone & $20.6 \%$ & 0.25 & Butyl butyryl lactate & $13.7 \%$ & 1.01 \\
\hline Butyric acid & $20.3 \%$ & 0.26 & 2,3-Pentanedione & $13.3 \%$ & 1.17 \\
\hline Ethyl isovalerate & $20.2 \%$ & 0.90 & $\gamma$-Undecalactone & $13.3 \%$ & 0.67 \\
\hline Acetic acid & $19.4 \%$ & 0.14 & Benzyl benzoate & $13.1 \%$ & 0.46 \\
\hline Benzyl acetate & $18.5 \%$ & 1.00 & Isoamyl acetate & $13.1 \%$ & 2.20 \\
\hline
\end{tabular}




\begin{tabular}{|c|c|c|c|c|c|}
\hline & & & & & \\
\hline Isoamyl isovalerate & $17.6 \%$ & 0.20 & Methyl- $\alpha$-ionone & $12.8 \%$ & 1.77 \\
\hline Benzaldehyde & $17.2 \%$ & 0.19 & Ethyl lactate & $12.6 \%$ & 0.67 \\
\hline Lemon oil & $17.2 \%$ & 6.10 & Methyl-thio- & $11.9 \%$ & 0.06 \\
\hline Benzyl alcohol & $16.0 \%$ & 2.82 & methylpyrazine & & \\
\hline Sucralose & $16.0 \%$ & 8.84 & $\beta$-Ionone & $11.1 \%$ & 0.42 \\
\hline Citral & $15.1 \%$ & 0.61 & Hexyl acetate & $10.8 \%$ & 0.60 \\
\hline Methyl cyclopentenolone & $14.5 \%$ & 0.46 & Propenyl guaethol & $10.2 \%$ & 1.00 \\
\hline Isoamyl butyrate & $14.2 \%$ & 1.64 & \multicolumn{3}{|c|}{ Category: Candy ( $n=34$ flavorings) } \\
\hline$\beta$-Ionone & $13.8 \%$ & 0.75 & Ethyl maltol & $39.4 \%$ & 0.50 \\
\hline Eugenol & $13.8 \%$ & 1.97 & Isoamyl acetate & $37.4 \%$ & 2.29 \\
\hline Ethyl propionate & $13.6 \%$ & 0.48 & Ethyl butyrate & $37.2 \%$ & 1.13 \\
\hline Hexanoic acid & $13.6 \%$ & 0.26 & Vanillin & $35.2 \%$ & 1.07 \\
\hline$\gamma$-Undecalactone & $13.5 \%$ & 0.41 & Ethyl acetate & $35.0 \%$ & 0.39 \\
\hline 4-(4-Hydroxyphenyl)- & $13.2 \%$ & 3.11 & Maltol & $26.2 \%$ & 0.50 \\
\hline 2-butanone & & & cis-3-Hexenol & $23.2 \%$ & 1.00 \\
\hline Lime oil & $13.1 \%$ & 27.50 & Ethyl 2-methyl butyrate & $22.9 \%$ & 0.17 \\
\hline$\alpha$-Ionone & $12.9 \%$ & 1.00 & Ethyl vanillin & $21.6 \%$ & 0.28 \\
\hline$\alpha$-Terpineol & $12.9 \%$ & 0.74 & Linalool & $21.0 \%$ & 0.66 \\
\hline Cinnamaldehyde & $12.5 \%$ & 3.31 & Butyric acid & $20.5 \%$ & 0.32 \\
\hline Limonene & $12.5 \%$ & 4.50 & Ethyl hexanoate & $20.1 \%$ & 0.11 \\
\hline cis-3-Hexenyl acetate & $12.3 \%$ & 0.14 & $\gamma$-Decalactone & $19.9 \%$ & 0.24 \\
\hline Piperonal & $11.9 \%$ & 0.20 & Ethyl isovalerate & $19.5 \%$ & 0.17 \\
\hline Hexyl acetate & $11.4 \%$ & 0.60 & Benzaldehyde & $17.9 \%$ & 0.10 \\
\hline Allyl hexanoate & $10.9 \%$ & 1.00 & Furaneol & $17.6 \%$ & 1.21 \\
\hline Anisaldehyde & $10.6 \%$ & 0.11 & Benzyl acetate & $17.3 \%$ & 1.00 \\
\hline$\gamma$-Nonalactone & $10.3 \%$ & 0.76 & 4-(4-Hydroxyphenyl)- & $17.0 \%$ & 1.00 \\
\hline 2-Methyl butyric acid & $10.2 \%$ & 0.17 & 2-butanone & & \\
\hline \multicolumn{3}{|c|}{ Category: Fruit-berries ( $n=36$ flavorings) } & Benzyl alcohol & $16.7 \%$ & 0.68 \\
\hline Ethyl butyrate & $52.4 \%$ & 6.21 & $\gamma$-Undecalactone & $16.0 \%$ & 0.20 \\
\hline cis-3-Hexenol & $41.2 \%$ & 2.33 & Hexyl acetate & $16.0 \%$ & 1.00 \\
\hline Vanillin & $39.1 \%$ & 3.05 & Isoamyl butyrate & $15.7 \%$ & 1.28 \\
\hline Furaneol & $36.8 \%$ & 3.26 & Isoamyl isovalerate & $14.8 \%$ & 0.39 \\
\hline Ethyl acetate & $36.0 \%$ & 1.36 & Methyl cinnamate & $13.9 \%$ & 0.18 \\
\hline Ethyl 2-methyl butyrate & $35.7 \%$ & 2.51 & Methyl cyclopentenolone & $13.3 \%$ & 0.14 \\
\hline Acetic acid & $33.5 \%$ & 2.31 & Hexanoic acid & $12.9 \%$ & 0.22 \\
\hline Ethyl maltol & $33.5 \%$ & 3.34 & 2-Methyl butyric acid & $12.6 \%$ & 0.30 \\
\hline$\gamma$-Decalactone & $33.1 \%$ & 0.91 & Citral & $12.3 \%$ & 0.75 \\
\hline Maltol & $32.7 \%$ & 1.21 & Acetic acid & $12.2 \%$ & 0.40 \\
\hline 4-(4-hydroxyphenyl)- & $32.6 \%$ & 1.78 & $\beta$-Ionone & $12.0 \%$ & 0.66 \\
\hline 2-butanone & & & cis-3-Hexenyl acetate & $11.4 \%$ & 0.26 \\
\hline Linalool & $31.1 \%$ & 0.92 & Ethyl propionate & $10.6 \%$ & 0.20 \\
\hline
\end{tabular}




\begin{tabular}{|c|c|c|c|c|c|}
\hline Hexanoic acid & $26.3 \%$ & 0.60 & Eugenol & $10.6 \%$ & 0.45 \\
\hline 2-Methyl butyric acid & $25.7 \%$ & 2.79 & Lemon oil & $10.4 \%$ & 0.13 \\
\hline Butyric acid & $25.5 \%$ & 0.85 & \multicolumn{3}{|c|}{ Category: Other sweets ( $n=20$ flavorings) } \\
\hline Ethyl hexanoate & $24.2 \%$ & 0.81 & Vanillin & $61.4 \%$ & 30.00 \\
\hline Ethyl isovalerate & $23.8 \%$ & 0.65 & Ethyl maltol & $37.5 \%$ & 28.04 \\
\hline Methyl cinnamate & $23.4 \%$ & 0.92 & Ethyl vanillin & $35.0 \%$ & 19.68 \\
\hline Isoamyl acetate & $22.0 \%$ & 1.00 & Maltol & $26.0 \%$ & 1.30 \\
\hline cis-3-Hexenyl acetate & $21.5 \%$ & 1.00 & Piperonal & $24.4 \%$ & 3.18 \\
\hline Benzyl acetate & $19.6 \%$ & 0.74 & Methyl cyclopentenolone & $22.0 \%$ & 5.65 \\
\hline$\gamma$-Undecalactone & $19.1 \%$ & 0.21 & $\delta$-Decalactone & $18.9 \%$ & 0.71 \\
\hline Benzyl alcohol & $19.0 \%$ & 1.02 & Ethyl butyrate & $18.6 \%$ & 0.51 \\
\hline Hexyl acetate & $18.7 \%$ & 0.41 & $\gamma$-Nonalactone & $16.2 \%$ & 0.75 \\
\hline Ethyl vanillin & $18.6 \%$ & 2.92 & Furaneol & $15.1 \%$ & 2.22 \\
\hline$\beta$-Ionone & $17.4 \%$ & 0.36 & Isoamyl isovalerate & $13.7 \%$ & 1.11 \\
\hline Ethyl propionate & $17.1 \%$ & 0.32 & 2,3,5-Trimethylpyrazine & $13.1 \%$ & 0.25 \\
\hline Benzaldehyde & $16.1 \%$ & 0.15 & Anisaldehyde & $13.1 \%$ & 0.50 \\
\hline$\alpha$-Ionone & $16.0 \%$ & 0.40 & Ethyl acetate & $13.0 \%$ & 0.57 \\
\hline Menthol & $15.7 \%$ & 9.12 & $\gamma$-Decalactone & $12.9 \%$ & 0.30 \\
\hline Dimethyl sulfide & $14.1 \%$ & 0.20 & Butyric acid & $12.6 \%$ & 0.77 \\
\hline Isoamyl isovalerate & $13.1 \%$ & 0.96 & Acetoin & $12.0 \%$ & 1.15 \\
\hline Isoamyl butyrate & $12.8 \%$ & 0.36 & Guaiacol & $11.1 \%$ & 0.04 \\
\hline$\delta$-Decalactone & $11.6 \%$ & 0.13 & $\delta$-Dodecalactone & $10.0 \%$ & 0.40 \\
\hline Sucralose & $11.3 \%$ & 12.15 & Veratraldehyde & $10.0 \%$ & 2.96 \\
\hline Propionic acid & $11.0 \%$ & 3.00 & \multicolumn{3}{|c|}{ Category: Other flavors ( $n=34$ flavorings) } \\
\hline \multicolumn{3}{|c|}{ Category: Fruit-citrus ( $n=31$ flavorings) } & Linalool & $30.5 \%$ & 3.00 \\
\hline Ethyl maltol & $32.9 \%$ & 5.98 & Ethyl butyrate & $25.2 \%$ & 6.00 \\
\hline Ethyl butyrate & $31.1 \%$ & 2.85 & Ethyl acetate & $23.2 \%$ & 8.00 \\
\hline Vanillin & $31.0 \%$ & 6.00 & Maltol & $23.2 \%$ & 4.10 \\
\hline Ethyl acetate & $26.3 \%$ & 0.66 & Ethyl 2-methyl butyrate & $22.5 \%$ & 5.90 \\
\hline Linalool & $25.8 \%$ & 1.56 & Vanillin & $21.9 \%$ & 21.00 \\
\hline Citral & $23.4 \%$ & 2.81 & Acetic acid & $21.2 \%$ & 0.70 \\
\hline Lemon oil & $21.9 \%$ & 12.00 & Isoamyl acetate & $20.5 \%$ & 6.00 \\
\hline Maltol & $20.3 \%$ & 0.48 & cis-3-Hexenyl acetate & $19.2 \%$ & 0.30 \\
\hline Ethyl vanillin & $19.9 \%$ & 16.00 & cis-3-Hexenol & $18.5 \%$ & 1.80 \\
\hline Lime oil & $19.4 \%$ & 9.66 & Ethyl acetoacetate & $18.5 \%$ & 0.20 \\
\hline$\alpha$-Terpineol & $19.0 \%$ & 0.26 & Ethyl hexanoate & $18.5 \%$ & 0.20 \\
\hline Furaneol & $18.4 \%$ & 1.50 & Butyric acid & $17.2 \%$ & 1.95 \\
\hline cis-3-Hexenol & $17.6 \%$ & 0.77 & Hexyl acetate & $17.2 \%$ & 8.00 \\
\hline Limonene & $17.1 \%$ & 13.00 & Isoamyl butyrate & $17.2 \%$ & 5.20 \\
\hline Orange oil & $16.1 \%$ & 1.74 & cis-3-Hexenyl butyrate & $15.2 \%$ & 0.10 \\
\hline$\gamma$-Decalactone & $15.9 \%$ & 0.24 & Ethyl isovalerate & $15.2 \%$ & 0.10 \\
\hline
\end{tabular}




\begin{tabular}{|c|c|c|c|c|c|}
\hline & \\
\hline Benzyl alcohol & $15.3 \%$ & 1.20 & $\gamma$-Decalactone & $15.2 \%$ & 1.20 \\
\hline Methyl cyclopentenolone & $14.9 \%$ & 8.57 & Hexanal & $15.2 \%$ & 0.02 \\
\hline Ethyl hexanoate & $14.7 \%$ & 2.62 & Linalyl acetate & $15.2 \%$ & 0.04 \\
\hline$\delta$-Dodecalactone & $14.1 \%$ & 1.13 & Furaneol & $13.9 \%$ & 7.40 \\
\hline Ethyl 2-methyl butyrate & $13.1 \%$ & 0.75 & Myrcene & $13.2 \%$ & 25.60 \\
\hline Acetic acid & $12.7 \%$ & 1.10 & Anisaldehyde & $12.6 \%$ & 1.80 \\
\hline Sucralose & $12.4 \%$ & 13.88 & Dihydrocoumarin & $12.6 \%$ & 30.00 \\
\hline$\gamma$-Nonalactone & $12.1 \%$ & 2.05 & Ethyl maltol & $12.6 \%$ & 17.70 \\
\hline Ethyl propionate & $11.1 \%$ & 1.30 & Limonene & $12.6 \%$ & 6.97 \\
\hline 4-(4-Hydroxyphenyl)- & $10.6 \%$ & 1.00 & 2-Phenylethanol & $11.9 \%$ & 0.10 \\
\hline 2-butanone & & & Citronellol & $11.9 \%$ & 6.00 \\
\hline Butyric acid & $10.3 \%$ & 0.10 & 2-Methyl butyric acid & $11.3 \%$ & 1.10 \\
\hline Hexanoic acid & $10.3 \%$ & 0.68 & Benzaldehyde & $11.3 \%$ & 6.60 \\
\hline Isoamyl isovalerate & $10.3 \%$ & 0.67 & $\beta$-Caryophyllene & $11.3 \%$ & 25.60 \\
\hline 2-Methyl butyric acid & $10.2 \%$ & 2.64 & Hexanoic acid & $11.3 \%$ & 0.20 \\
\hline Ethyl isovalerate & $10.0 \%$ & 0.20 & Methyl cinnamate & $11.3 \%$ & 0.40 \\
\hline \multicolumn{3}{|c|}{ Category: Fruit-tropical $(n=39$ flavorings) } & $\beta$-Damascenone & $10.6 \%$ & 0.10 \\
\hline Ethyl butyrate & $38.5 \%$ & 2.42 & \multicolumn{3}{|c|}{ Category: Unflavored ( $n=0$ flavorings) } \\
\hline Vanillin & $36.5 \%$ & 2.75 & NA & NA & NA \\
\hline
\end{tabular}

Prevalence is reported as the number of e-liquids containing the respective flavoring as percentage of the total number of e-liquids. The final column shows the median concentration of the flavoring ingredient for the respective flavor category. The 29 "category-specific" flavorings that were added to more than $10 \%$ of the e-liquids in a single category are marked in light grey. 
Appendix Table A4.3: Flavor descriptions, retrieved from a commercial flavor database ${ }^{34}$, of the ingredients listed in the main text of the manuscript. Ingredients are presented in alphabetical order.

\begin{tabular}{|c|c|c|}
\hline Flavoring ingredient & Flavor description & $\begin{array}{l}\text { Flavor } \\
\text { category* }\end{array}$ \\
\hline 3,5-Trimethylpyrazine & Baked potato, roasted nut, cocoa, coffee, burnt & \\
\hline 2,5-Dimethylpyrazine & Chocolate, roasted nuts, earthy & Nuts \\
\hline 2-3-Hexanedione & Creamy, sweet buttery; butter-cheese, fruity, caramellic & Nuts \\
\hline $\begin{array}{l}\text { 4,5-Dimethyl-3-hydroxy- } \\
\text { 2,5-dihydrofuran-2-one }\end{array}$ & Powerful caramel aroma; sweet burnt taste & Nuts \\
\hline 4-Methyl-5-thiazole ethanol & In dilution, meaty-beef like with nutty note & Dessert \\
\hline 5-Methyl furfural & Sweet spicy, bready, nutty, caramellic & Nuts \\
\hline Acetic acid $^{\circ}$ & Pungent, sour, acid, vinegar & \\
\hline Acetoin & Creamy-buttery, yogurt-like & \\
\hline Anethole & Sweet, herbaceous, anise (artificial licorice) & Spices \\
\hline Anisyl alcohol & $\begin{array}{l}\text { Sweet, fruity, floral, balsamic anisic-vanilla-creamy- } \\
\text { coumarinic }\end{array}$ & Dessert \\
\hline Benzaldehyde $^{o}$ & Bitter almond oil, sweet cherry & \\
\hline Benzyl alcohol $^{\circ}$ & Faint, sweet, almond fruity, somewhat chemical & \\
\hline Benzyl benzoate & Faint, sweet, balsamic with slight bitter, fruity notes & Dessert \\
\hline Butyric acid $^{\circ}$ & Tropical fruity floral, plum, apricot-pear-like & \\
\hline Cinnamaldehyde & Spicy, cinnamon-cassia-like with sweet warm (hot) taste & Other beverages \\
\hline Cis-3-hexenol ${ }^{\circ}$ & Strong, fresh, green, grassy & \\
\hline$\delta$-Decalactone ${ }^{\mathrm{O}}$ & Sweet, creamy, milky, peach, nut, buttery & \\
\hline Dimethyl sulfide & $\begin{array}{l}\text { Pungent, cabbage, cooked vegetable odor; corn-like on } \\
\text { dilution }\end{array}$ & Fruit, berries \\
\hline Ethyl 2-methyl butyrate ${ }^{o}$ & Strong, green, fruity, apple with strawberry notes & \\
\hline Ethyl acetate $^{\mathrm{o}}$ & Ethereal, sharp, wine-brandy-like & \\
\hline Ethyl butyrate $^{o}$ & $\begin{array}{l}\text { Ethereal, fruity with buttery-pineapple-banana, ripe fruit, } \\
\text { and juicy notes }\end{array}$ & \\
\hline Ethyl heptanoate & Strong, fruity, winey, cognac-like & Alcohol \\
\hline Ethyl hexanoate $^{\circ}$ & $\begin{array}{l}\text { Strong, fruity, pineapple, banana with strawberry, pear, } \\
\text { and tropical notes }\end{array}$ & \\
\hline Ethyl maltol ${ }^{\circ}$ & Sweet, fruity-caramellic, cotton candy & \\
\hline Ethyl nonanoate & Fatty-waxy, oily, wine-cognac, grape, tropical, nut-like & Alcohol \\
\hline Ethyl propionate $^{\circ}$ & Strong, ethereal, fruity, rum-like & \\
\hline Ethyl vanillin ${ }^{\circ}$ & Intense, sweet, creamy, vanilla-like & \\
\hline Eucalyptol & Strong, camphoraceous, cool, fresh & Menthol/mint \\
\hline Furaneol $^{\circ}$ & Fruity, caramelized, roasted, pineapple-strawberry & \\
\hline Hexanoic acid $^{\circ}$ & Heavy, fatty, cheesey-sweaty & \\
\hline Hexyl acetate ${ }^{\circ}$ & Sweet, fruity, pear-apple, green, banana & \\
\hline Isoamyl acetate $^{\circ}$ & Sweet, fruity, banana, pear & \\
\hline Isoamyl alcohol & $\begin{array}{l}\text { Breathtaking, alcoholic odor; in dilution a winey-brandy } \\
\text { taste }\end{array}$ & Alcohol \\
\hline
\end{tabular}




\begin{tabular}{|c|c|c|}
\hline Isoamyl isovalerate $^{\circ}$ & $\begin{array}{l}\text { Fruity, green-apple, pineapple, tropical, mango, apricot, } \\
\text { cognac }\end{array}$ & \\
\hline Isobutyl acetate & Fruity, banana-apple-pear-pineapple & Fruit, other \\
\hline Lactic acid & Weak, sour, buttermilk & Alcohol \\
\hline Linalool $^{\circ}$ & Sweet floral-woody with slight citrus notes & \\
\hline Maltol $^{\circ}$ & Sweet, fruity, berry, strawberry, caramellic & \\
\hline Menthol $^{\circ}$ & $\begin{array}{l}\text { String trigeminal cooling sensation with a slight mint } \\
\text { note }\end{array}$ & \\
\hline Menthone & Minty-herbaceous (not green); dry woody notes & Menthol/mint \\
\hline Methyl cyclopentenolone $^{\circ}$ & Very strong, caramellic-maple, lovage & \\
\hline Methyl- $\alpha$-ionone & $\begin{array}{l}\text { Orris, violet, woody, floral, oily with woody raspberry } \\
\text { notes }\end{array}$ & Dessert \\
\hline Methyl-thio-methyl-pyrazine & Roasted nut, burnt, meaty & Dessert \\
\hline Orange oil & Orange & Fruit, citrus \\
\hline Peppermint oil & Peppermint & Menthol/mint \\
\hline Piperonal & $\begin{array}{l}\text { Sweet, floral-cherry (heliotrope); sweet cherry-vanilla } \\
\text { taste }\end{array}$ & \\
\hline Propenyl guaethol & Sweet, vanilla, creamy, phenolic, anisic flavor & Dessert \\
\hline Propionic acid & Pungent, sour milk, cheese & Fruit, berries \\
\hline Trans-2-hexenal & Green, fruity, fresh, apple with leafy and grassy notes & Fruit, other \\
\hline Trans-anethole & Sweet, herbaceous, anise (artificial licorice) & Spices \\
\hline Triethyl citrate & $\begin{array}{l}\text { Weak, sweet, winey-fruity-plum-like odor; slight bitter } \\
\text { taste }\end{array}$ & Nuts \\
\hline Vanillin $^{\circ}$ & Sweet, powerful, creamy, vanilla-like & \\
\hline$\beta$-damascone & Blackcurrant, plum, rose, honey, tobacco & Tobacco \\
\hline$\gamma$-Decalactone ${ }^{o}$ & Coconut-peach & \\
\hline$\gamma$-Dodecalactone & Fatty, fruity, peach odor & Nuts \\
\hline$\gamma$-Hexalactone & Coumarin-like, sweet, creamy note & Dessert \\
\hline$\gamma$-Undecalactone ${ }^{\circ}$ & Strong fatty, peach-apricot & \\
\hline
\end{tabular}

* Flavor category that the ingredient is specific to ("category-specific flavorings"). A flavoring ingredient added to more than $10 \%$ of the e-liquids in a single category was considered "category-specific".

${ }^{\circ}$ The 25 flavoring ingredients that were most frequently added to e-liquids from the EU-CEG dataset. These data are also presented in Table 4.1 of the main manuscript. 
ing

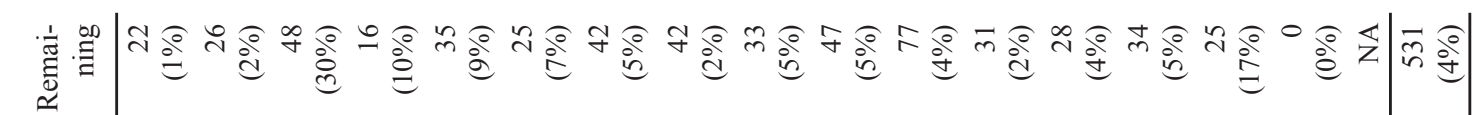

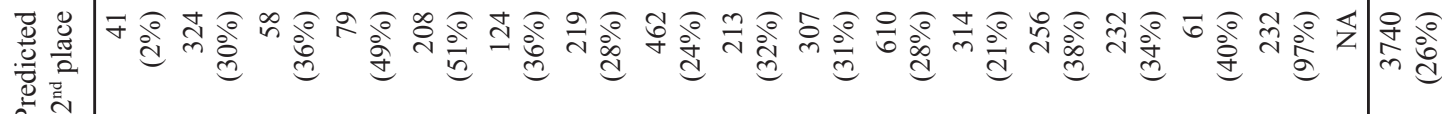
范范茫

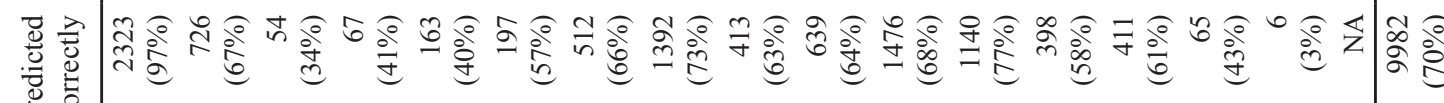
- 2

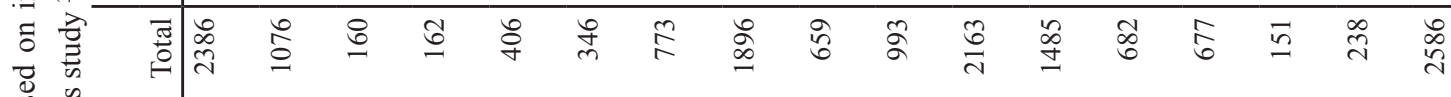

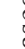
2 d 荧 ๖ั 赵 $\pi$ t

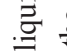
营

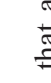
. \begin{tabular}{l|lllllllllllllllll}
0 & 0 & 0 & 0 & 0 & 0 & 0 & 0 & 0 & 0 & 0 & 0 & 0 & 0 & 0 & 0 & 0 & 0
\end{tabular}

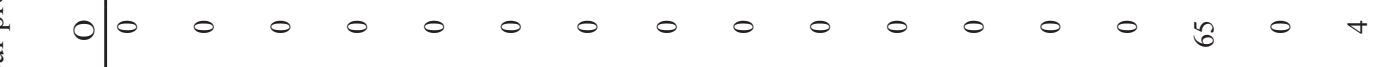

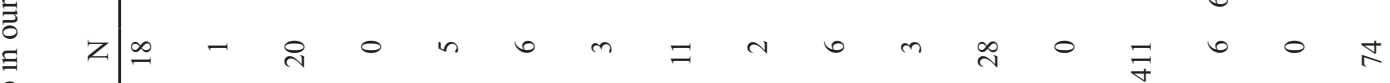

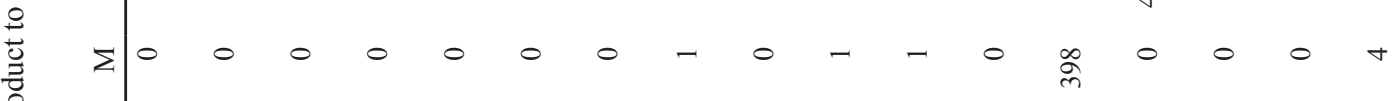

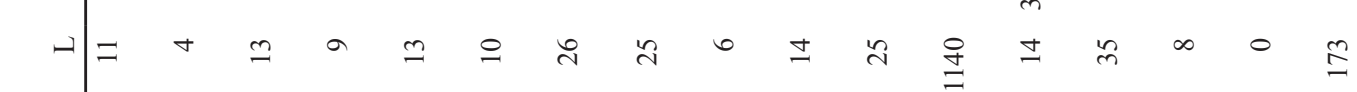

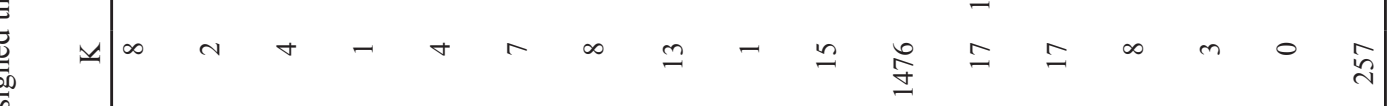

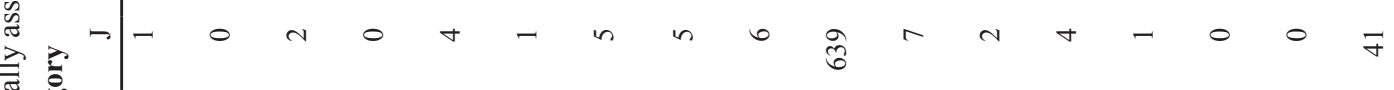
莺

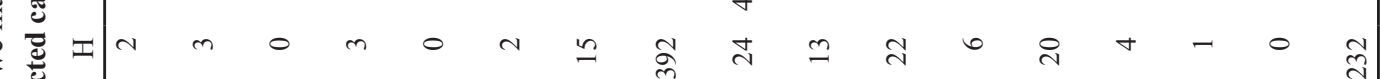
:

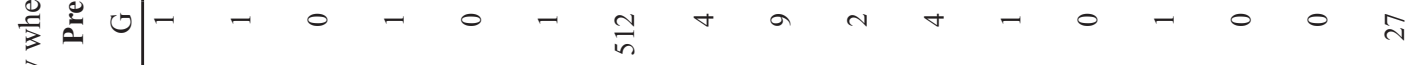
를 幽 강 惫 $2<$

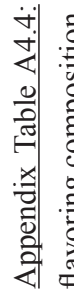

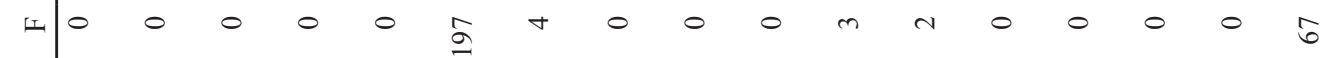
I

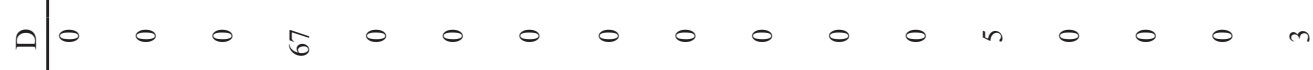

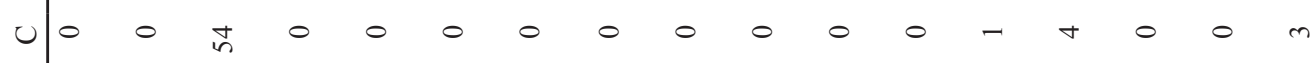
$m$ ก

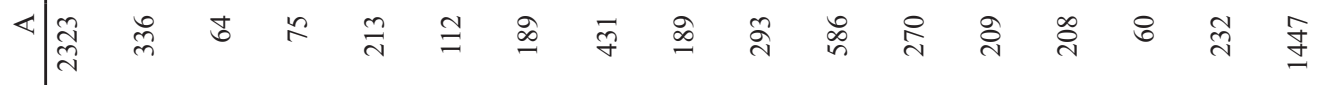

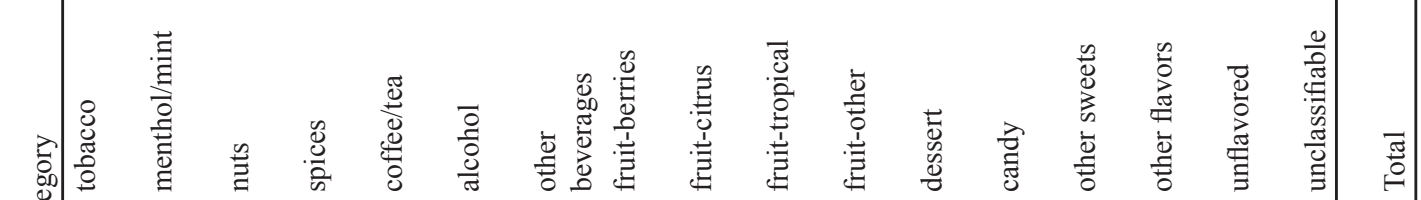
Uే ๑ 0 川 I 


\section{References}

1. Filippidis FT, Laverty AA, Gerovasili V, Vardavas CI. Two-year trends and predictors of e-cigarette use in 27 European Union member states. Tob Control. 2017;26(1):98.

2. McMillen RC, Gottlieb MA, Shaefer RM, Winickoff JP, Klein JD. Trends in Electronic Cigarette Use Among U.S. Adults: Use is Increasing in Both Smokers and Nonsmokers. Nicotine Tob Res. 2015;17(10):1195-1202.

3. Zare S, Nemati M, Zheng Y. A systematic review of consumer preference for e-cigarette attributes: Flavor, nicotine strength, and type. PLoS One. 2018;13(3):e0194145.

4. Kong G, Morean ME, Cavallo DA, Camenga DR, Krishnan-Sarin S. Reasons for Electronic Cigarette Experimentation and Discontinuation Among Adolescents and Young Adults. Nicotine Tob Res. 2015; 17(7):847-854.

5. Harrell MB, Weaver SR, Loukas A, et al. Flavored e-cigarette use: Characterizing youth, young adult, and adult users. Prev Med Reports. 2017;5:33-40.

6. Pepper JK, Ribisl KM, Brewer NT. Adolescents' interest in trying flavoured e-cigarettes. Tob Control. 2016;25(Suppl 2):ii62-ii66.

7. Havermans A, Krüsemann EJZ, Pennings J, de Graaf K, Boesveldt S, Talhout R. Nearly 20000 e-liquids and 250 unique flavour descriptions: an overview of the Dutch market based on information from manufacturers. Tob Control. 2019:tobaccocontrol-2019-055303.

8. Schneller LM, Bansal-Travers M, Goniewicz ML, McIntosh S, Ossip D, O'Connor RJ. Use of flavored electronic cigarette refill liquids among adults and youth in the US-Results from Wave 2 of the Population Assessment of Tobacco and Health Study (2014-2015). PLoS One. 2018;13(8):e0202744.

9. Romijnders K, van Osch L, de Vries H, Talhout R. Perceptions and Reasons Regarding E-Cigarette Use among Users and Non-Users: A Narrative Literature Review. Int J Environ Res Public Health. 2018;15(6).

10. Prochaska JJ. The public health consequences of e-cigarettes: a review by the National Academies of Sciences. A call for more research, a need for regulatory action. Addiction. 2019;114(4):587-589.

11. Chen J, Bullen C, Dirks K. A Comparative Health Risk Assessment of Electronic Cigarettes and Conventional Cigarettes. Int J Environ Res Public Health. 2017;14(4).

12. Goniewicz ML, Gawron M, Smith DM, Peng M, Jacob P, 3rd, Benowitz NL. Exposure to Nicotine and Selected Toxicants in Cigarette Smokers Who Switched to Electronic Cigarettes: A Longitudinal Within-Subjects Observational Study. Nicotine Tob Res. 2017;19(2):160-167.

13. Levy DT, Borland R, Lindblom EN, et al. Potential deaths averted in USA by replacing cigarettes with e-cigarettes. Tob Control. 2018;27(1):18-25.

14. Margham J, McAdam K, Forster M, et al. Chemical Composition of Aerosol from an E-Cigarette: A Quantitative Comparison with Cigarette Smoke. Chem Res Toxicol. 2016;29(10):1662-1678.

15. Cullen KA, Ambrose BK, Gentzke AS, Apelberg BJ, Jamal A, King BA. Notes from the Field: Use of Electronic Cigarettes and Any Tobacco Product Among Middle and High School Students - United States, 2011-2018. MMWR Morb Mortal Wkly Rep. 2018;67(45):1276-1277.

16. Miech R, Johnston L, O’Malley PM, Bachman JG, Patrick ME. Adolescent Vaping and Nicotine Use in 2017-2018 - U.S. National Estimates. N Engl J Med. 2019;380(2):192-193.

17. Central Agency for Statistics (CBS), National Institute for Public Health and the Environment (RIVM), Trimbos-instituut. Roken: Cijfers \& Context. 2018; https://www.volksgezondheidenzorg. info/onderwerp/roken/cijfers-context/huidige-situatie-jongeren. 
18. McRobbie H. Modelling the Population Health Effects of E-Cigarettes Use: Current Data Can Help Guide Future Policy Decisions. Nicotine Tob Res. 2017;19(2):131-132.

19. Layden JE, Ghinai I, Pray I, et al. Pulmonary Illness Related to E-Cigarette Use in Illinois and Wisconsin - Final Report. N Engl J Med. 2020;382(10):903-916.

20. Eddingsaas N, Pagano T, Cummings C, Rahman I, Robinson R, Hensel E. Qualitative Analysis of E-Liquid Emissions as a Function of Flavor Additives Using Two Aerosol Capture Methods. Int J Environ Res Public Health. 2018;15(2).

21. Klager S, Vallarino J, MacNaughton P, Christiani DC, Lu Q, Allen JG. Flavoring Chemicals and Aldehydes in E-Cigarette Emissions. Environ Sci Technol. 2017;51(18):10806-10813.

22. Goniewicz ML. Carcinogens and toxicants in e-cigarettes. Cancer Prev Res. 2013;6(11).

23. Soneji S, Barrington-Trimis JL, Wills TA, et al. Association Between Initial Use of e-Cigarettes and Subsequent Cigarette Smoking Among Adolescents and Young Adults: A Systematic Review and Meta-analysis. JAMA Pediatr. 2017;171(8):788-797.

24. US Food and Drug Administration (FDA). Trump Administration Combating Epidemic of Youth E-Cigarette Use with Plan to Clear Market of Unauthorized, Non-Tobacco-Flavored E-Cigarette Products [press release]. Silver Spring, MD, 2019.

25. Girvalaki C, Tzatzarakis M, Kyriakos CN, et al. Composition and chemical health hazards of the most common electronic cigarette liquids in nine European countries. Inhal Toxicol. 2018;30(910):361-369.

26. Hahn J, Monakhova YB, Hengen J, et al. Electronic cigarettes: overview of chemical composition and exposure estimation. Tob Induc Dis. 2014;12(1):23.

27. Hutzler C, Paschke M, Kruschinski S, Henkler F, Hahn J, Luch A. Chemical hazards present in liquids and vapors of electronic cigarettes. Arch Toxicol. 2014;88(7):1295-1308.

28. Lisko JG, Tran H, Stanfill SB, Blount BC, Watson CH. Chemical Composition and Evaluation of Nicotine, Tobacco Alkaloids, $\mathrm{pH}$, and Selected Flavors in E-Cigarette Cartridges and Refill Solutions. Nicotine Tob Res. 2015;17(10):1270-1278.

29. Tierney PA, Karpinski CD, Brown JE, Luo W, Pankow JF. Flavour chemicals in electronic cigarette fluids. Tob Control. 2016;25(e1):e10-15.

30. Krüsemann EJZ, Boesveldt S, de GraafK, Talhout R. An E-Liquid Flavor Wheel: A Shared Vocabulary Based on Systematically Reviewing E-Liquid Flavor Classifications in Literature. Nicotine Tob Res. 2019;21(10):1310-1319.

31. European Commission. EU Common Entry Gate (EU-CEG). 2016; https://ec.europa.eu/health/ euceg/introduction_en.

32. Breiman L. Random Forests. Mach Learn. 2001;45(1):5-32.

33. The European Commission. Commission Implementing Decision (EU) 2015/2183 of 24 November 2015 establishing a common format for the notification of electronic cigarettes and refill containers (notified under document C(2015) 8087). Official Journal of the European Union. 2015;L 309:15-27.

34. Leffingwell \& Associates. Flavor-Base 9 - Tobacco Version for Windows XP/Vista/7\&8. 2013.

35. Van Nierop LE, Pennings JLA, Schenk E, Kienhuis AS, Talhout R. Analysis of Manufacturer's Information on Tobacco Product Additive Use. Tob Regul Sci. 2019;5(2):182-205.

36. Ferris Wayne G, Connolly GN. Application, function, and effects of menthol in cigarettes: a survey of tobacco industry documents. Nicotine Tob Res. 2004;6 Suppl 1:S43-54.

37. Havermans A, Krüsemann EJZ, Pennings J, de Graaf K, Boesveldt S, Talhout R. Nearly 20000 
e-liquids and 250 unique flavour descriptions: an overview of the Dutch market based on information from manufacturers. Tob Control. 2019:tobaccocontrol-2019-055303. 



\section{CHAPTER 5}

\section{GC-MS analysis of e-cigarette refill solutions: A comparison of flavoring composition between flavor categories}

Erna J.Z. Krüsemann

Jeroen L.A. Pennings

Johannes W.J.M. Cremers

Frank Bakker

Sanne Boesveldt

Reinskje Talhout

Published in Journal of Pharmaceutical and Biomedical Analysis. 2020;188:113364. https://doi.org/10.1016/j.jpba.2020.113364. 


\begin{abstract}
Objectives: Electronic cigarette refill solutions (e-liquids) are available in various flavor descriptions that can be categorized as fruit, tobacco, and more. Flavors increase sensory appeal, thereby stimulating e-cigarette use, and flavoring ingredients can contribute to e-cigarette toxicity. We aim to inform toxicologists, sensory scientists, and regulators by determining flavoring compounds in e-liquids with various flavors, and compare results between flavor categories. Methods: Gas chromatography - mass spectrometry (GC-MS) was used to identify 79 flavorings in 320 e-liquids, classified in 15 flavor categories. Ten flavorings highly prevalent in e-liquids according to information from manufacturers were quantified. Flavoring prevalence was defined as the number of e-liquids with the flavoring as percentage of the total number of e-liquids. The method was validated in terms of specificity, linearity, repeatability, recovery, and sensitivity. Results: The mean number of flavorings per e-liquid was $6 \pm 4$. Flavoring prevalence was highest for vanillin (creamy/vanilla flavor), ethyl butyrate (ethereal/fruity), and cis-3hexenol (fresh/green). Based on similarities in flavoring prevalence, four clusters of categories were distinguished: (1) fruit, candy, alcohol, beverages; (2) dessert, coffee/tea, nuts, sweets; (3) menthol/mint; and (4) spices, tobacco, and unflavored. Categories from cluster 4 generally had less flavorings per e-liquid than fruit, candy, alcohol, beverages (cluster 1) and dessert (cluster 2) $(p<0.05)$. Flavoring concentrations varied between e-liquids within the categories. Conclusions: We evaluated flavoring compositions of 320 e-liquids using a simple GC-MS method. Flavoring prevalence was similar within four clusters of typically fresh/sweet, warm/sweet, fresh/cooling, and non-sweet flavor categories. To compare flavoring concentrations between individual flavor categories, additional research is needed.
\end{abstract}




\section{Introduction}

Over the past years, the use of electronic cigarettes (e-cigarettes) has been increasing worldwide ${ }^{1,2}$. An important reason for experimentation with e-cigarettes is the variety of available flavors in e-cigarette refill solutions (e-liquids) ${ }^{3}$. Not surprisingly, e-liquid flavors are an important focus for marketeers: we showed that the Dutch e-liquid market (2017) alone already comprised no less than 245 unique flavor names ${ }^{4}$. Whereas e-cigarettes may be an appealing, less harmful substitute for tobacco smoking among adults ${ }^{5}$, particularly young people who currently do not smoke are attracted to e-liquid flavors and thereby prone to initiation of e-cigarette use ${ }^{6}$. Importantly, e-cigarettes cause users to inhale potentially toxic substances, and are therefore not safe ${ }^{7}$. Additionally, e-cigarettes often contain nicotine and thereby present a substantial risk of nicotine dependence. This makes regulation of e-cigarette flavors, to reduce appeal for those who would otherwise not smoke, currently an important topic of debate ${ }^{8}$.

One way to regulate e-liquid flavors is to restrict particular flavoring ingredients (from now on referred to as flavorings). For combustible cigarettes and roll-your-own tobacco, the European Commission created a list of 15 priority additives that have no health benefits, but may instead stimulate use of and addiction to an extremely harmful product ${ }^{9}$. Similarly, for e-cigarettes, regulators could focus on flavorings of which inhalation is known to be toxic. Banning these could directly reduce harm from exposure to e-cigarette emissions. Another option is to ban the most popular e-liquid flavorings, thereby reducing overall appeal of the product. This may result in decreased use and thereby decreased exposure to toxic constituents of e-cigarette emissions among vulnerable user groups such as non-smokers and youth.

As the composition of the e-liquid flavor is often not mentioned on the product label, research is needed to identify e-liquid flavorings in order to provide focus for toxicologists, sensory scientists, and regulators. Recently, we published a comprehensive overview of the most prevalent e-liquid flavoring additives across 16839 e-liquids based on information from manufacturers ${ }^{10}$. However, industry data may not always be present, complete, or correct. In addition, research has shown that e-liquid ingredients can react with one another to form new molecules ${ }^{11}$, which may make ingredients lists from manufacturers less suitable for risk assessment of the product. Therefore, additional chemical-analytical research is needed.

Gas chromatographic-mass spectrometric (GC-MS) methods are widely accepted for analysis of volatile compounds such as flavorings in several types of products, such as cheese ${ }^{12}$, wine ${ }^{13}$, olive oil ${ }^{14}$, as well as tobacco ${ }^{15}$. Several studies were performed to determine flavorings and other chemicals in e-cigarette aerosol ${ }^{16,17}$, for example to identify harmful and potentially harmful constituents that users are exposed to ${ }^{18}$. As there is a relation between constituents in e-cigarette aerosol generated by vaping machines and constituents in the e-liquid, qualitative and quantitative GC-MS methods have also been used to determine flavorings in e-liquids, e.g. ${ }^{16,19-23}$. However, data on e-liquid flavoring ingredients in relation to their marketed flavor descriptions are limited. Aszyck et al. ${ }^{22}$ compared e-liquid flavoring profiles between five different brands for five e-liquid flavors (tobacco, strawberry, cherry, menthol and apple). Our study provides new insights by comparing flavoring compositions of e-liquids in more than 200 different flavor descriptions across multiple flavor categories, and includes a larger number of e-liquids than any previous chemical-analytical study. Additionally, flavoring concentrations 
will be compared between flavor categories by quantifying the 10 most prevalent flavorings as reported by manufacturers in our previous study ${ }^{10}$. By identifying common e-liquid flavorings using GC-MS and comparing results between flavor categories, we aim to inform toxicologists, sensory scientists, and regulators regarding attractiveness and toxicity of e-liquids. 


\section{Methods}

\section{E-liquid samples}

Commercial e-liquids, all intended to be used as e-cigarette refill solutions, were purchased online from 9 different vendors in the Netherlands. Based on the product name and flavor-related descriptors on the vendor's Web site, the main e-liquid flavor was classified into the following 15 categories of the e-liquid flavor wheel: tobacco, menthol/mint, nuts, spices, coffee/tea, alcohol, other beverages, fruit-berries, fruit-citrus, fruit-tropical, fruit-other, dessert, candy, other sweets, and unflavored ${ }^{24}$. We selected a large variety of specific e-liquid flavors for an optimal representation of each main category. Selection of the specific e-liquid flavors was based on the subcategories identified in our previous analysis of e-liquid flavors on the Dutch market ${ }^{4}$ : we aimed to select a maximum of two e-liquids per subcategory (based on availability). Selecting the brand and vendor of an e-liquid flavor was based on availability; when an e-liquid flavor was available from multiple brands or vendors, selection was based on obtaining a large variety in brands rather than a large number of vendors. This resulted in a final sample of 320 e-liquids from 204 different subcategories, that is, with 204 unique flavor names. If an e-liquid was available in multiple nicotine concentrations, a randomized choice was made from one of the following three categories: zero $(0 \mathrm{mg} / \mathrm{mL})$, low $(1-8 \mathrm{mg} / \mathrm{mL})$, and high $(9-18 \mathrm{mg} / \mathrm{mL})$. None of the e-liquids contained nicotine salts. Propylene glycol to vegetable glycerin $(\mathrm{PG} / \mathrm{VG})$ ratios as declared are reported; Table 5.1 shows an overview of the sample characteristics. Samples were stored in their original package at room temperature, and analyzed directly after opening.

\section{Target flavorings}

A targeted approach was used to determine 79 flavorings and nicotine. Selection of target compounds was based on information from manufacturers ${ }^{10}$, target lists of previous chemicalanalytical studies on e-liquid flavors ${ }^{19-21}$, and additives found in flavored tobacco products ${ }^{15}$. We quantified the following 10 flavorings, as these were the most commonly added e-liquid flavorings according to information from manufacturers in the Netherlands ${ }^{10}$ : vanillin, ethyl maltol, ethyl butyrate, ethyl acetate, maltol, ethyl vanillin, furaneol, methyl cyclopentenolone, $\gamma$-decalactone, and cis-3-hexenol. The 79 target flavorings tested, including their flavor descriptions based on a commercial flavor database ${ }^{25}$, are listed in Appendix Table A5.1.

\section{Other chemicals}

Standards of the target compounds in analytical or food grade (purity $\geq 95-99 \%$ ) were used to optimize identification accuracy. All flavoring chemicals were purchased from Sigma-Aldrich (Zwijndrecht, the Netherlands); ethyl acetate was purchased from Alfa Aesar (Kandel, Germany). Ethanol absolute was obtained from Merck (Darmstadt, Germany). Nicotine (purity > 99\%) and the internal standards benzene-d6 (purity 100\%) and n-heptadecane (purity $>99 \%$ ) were obtained from Acros Organics, Sigma-Aldrich, and Merck, respectively. 
Table 5.1: Characteristics of the 320 e-liquids selected for this study.

\begin{tabular}{llll}
\hline Variable & & \multicolumn{2}{l}{ Number of e-liquids (\%) } \\
\hline Main flavor category & Tobacco & 32 & $(10 \%)$ \\
& Menthol/mint & 8 & $(3 \%)$ \\
& Nuts & 8 & $(3 \%)$ \\
& Spices & 12 & $(4 \%)$ \\
& Coffee/tea & 19 & $(6 \%)$ \\
& Alcohol & 25 & $(8 \%)$ \\
& Other beverages & 28 & $(9 \%)$ \\
& Fruit (berries) & 22 & $(7 \%)$ \\
& Fruit (citrus) & 11 & $(3 \%)$ \\
& Fruit (tropical) & 22 & $(7 \%)$ \\
& Fruit (other & 28 & $(9 \%)$ \\
& Dessert & 61 & $(19 \%)$ \\
& Candy & 29 & $(9 \%)$ \\
& Other sweets & 13 & $(4 \%)$ \\
& Unflavored & 2 & $(1 \%)$ \\
\hline Nicotine level (mg/mL) & None $(0 \mathrm{mg} / \mathrm{mL})$ & 121 & $(38 \%)$ \\
& Low (1-8 mg/mL) & 107 & $(33 \%)$ \\
& High $(9-18 \mathrm{mg} / \mathrm{mL})$ & 92 & $(29 \%)$ \\
\hline VG level & $<50 \%$ & 75 & $(23 \%)$ \\
& $=50 \%$ & 91 & $(28 \%)$ \\
& $>50 \%$ & 154 & $(48 \%)$ \\
\hline
\end{tabular}

Marketed flavor descriptions were used to classify the e-liquids in 15 main categories of the e-liquid flavor wheel ${ }^{24}$. General note: percentages may not add to $100 \%$ due to rounding.

$\mathrm{VG}$, vegetable glycerin.

\section{Standard solutions and test sample preparation}

For qualification of the flavorings and nicotine, the standards were individually dissolved in ethanol (ca. $5 \mu \mathrm{g} / \mathrm{mL}$ ). One solution of the internal standards benzene-d6 and n-heptadecane was prepared in ethanol (both $100.0 \mu \mathrm{g} / \mathrm{L}$ ). For quantification, 9 flavoring standards were dissolved as a mixture in the internal standard solution in 10 different concentrations; furaneol was dissolved separately (see Appendix Table A5.2 for the concentration ranges). All test samples were diluted with the internal standard solution in a 1:100 ratio in duplicate. The standard solution was stored in the refrigerator at $4 \square \mathrm{C}$ until usage.

\section{GC-MS conditions}

An Agilent 7890B GC system coupled with an Agilent 240 ion trap mass spectrometer was used, equipped with a 7693 auto-sampler and a G4513A injector. Compounds were chromatographically separated using an Inert Cap Aquatic-2 column $(60 \mathrm{~m} \times 250 \mu \mathrm{m}$ i.d., 1.4 $\mu \mathrm{m}$ film thickness; medium polarity), with helium as a carrier gas in a constant flow rate of $1 \mathrm{~mL} /$ 
min. The temperature program was set at $50^{\circ} \mathrm{C}$ (hold for $6 \mathrm{~min}$ ), then ramp to $250^{\circ} \mathrm{C}$ with $10^{\circ} \mathrm{C} /$ min (hold for $9 \mathrm{~min}$ ). Total run time was $35 \mathrm{~min}$. The injection volume was $1 \mu \mathrm{L}$ with a 10:1 split ratio. The injector temperature and temperature of the transfer line were set at $200^{\circ} \mathrm{C}$ and $260^{\circ} \mathrm{C}$, respectively. The MS operated in a positive electron impact (EI) mode with an electron energy of $70 \mathrm{eV}$. The ion source temperature was set at $260^{\circ} \mathrm{C}$. After each test sample, a blank sample containing the ethanol-based internal standard solution $(100 \mu \mathrm{g} / \mathrm{L})$ was included to control for carry-over effects.

Qualification of target flavorings and nicotine was performed in full scan mode covering $29-250 \mathrm{~m} / \mathrm{z}$, a range sufficiently broad to cover the analytes. Qualification was based on the retention times and the MS spectra of the individual standards (i.e., references) listed in Appendix Table A5.1. Appendix Figure A5.1 shows chromatograms of a standard mixture and two e-liquid samples. Acceptance criteria for positive identification were: a maximum deviation of \pm 0.2 minutes of the expected retention time, a maximum difference of $20 \%$ between the relative intensities of quantifier/qualifier in the e-liquid samples versus the standards, and a match of at least $70 \%$ between the sample and reference spectrum. To verify the presence of flavorings in each e-liquid sample, retention times and the mass spectra were confirmed using those of the standards.

The 10 flavorings of interest were quantified in e-liquids where the respective flavoring was positively identified, based on the quantifier ion (Appendix Table A5.1). Concentrations were reported for flavorings with a signal at least 10 times higher than noise, based on the average signal to noise ratio of two runs. Two internal standards that differed significantly in retention time, ranging from 13 (benzene-d6) to 31 (n-heptadecane) minutes, were selected, thereby spanning the range in which the components of interest eluted. N-heptadecane was used as a back-up in case there would be interference of the peak of the analyte with that of the primary internal standard. This was not the case, hence, only benzene-d6 was used for quantification of the flavorings.

\section{Method validation procedures}

To determine specificity, we compared the retention time - quantifier ion combination between each of the 79 target compounds, nicotine, and the internal standards. A criterium was set that compounds with the same quantifier ion should have a difference in retention time of $>0.4$ minutes. This was based on three times the highest absolute standard deviation of the retention times, which was 0.11 minutes for ethyl maltol. Linearity of the 10 specific calibration curves was assessed, in duplicate, by dissolving a mixture of 9 flavoring standards in the internal standard solution in 10 different concentrations $(\sim 10-100 \mu \mathrm{g} / \mathrm{mL})$; furaneol was dissolved separately. To determine repeatability of the retention times and peak areas for the 10 flavorings and the two internal standards, two solutions of PG and VG were made in 30:70 and 70:30 ratio, respectively. A mixture of 9 flavorings ( $100 \mu \mathrm{g} / \mathrm{mL}$ dissolved in the ethanol-based internal standard solution) was added to these solutions in two different concentrations (20 and $80 \mu \mathrm{g} / \mathrm{mL}$ ); furaneol was dissolved separately. Each of the 4 solutions and a blank sample containing only the internal standard solution $(100 \mu \mathrm{g} / \mathrm{L})$ were injected in the GC-MS system 6 times. For each compound, we aimed for a relative standard deviation (RSD) of less than $1 \%$ and $10 \%$ for the retention time and peak area, respectively. To determine recovery, the same solutions of PG/VG (70/30 and 
30/70) and flavorings ( 20 and $80 \mu \mathrm{g} / \mathrm{ml}$ ) were used. For each component, results of 6 injections were averaged. The recovery was defined as the determined concentration as percentage of the added concentration of the respective flavoring. For sensitivity of the 10 flavorings, flavoring standards with a concentration of 10 or $30 \mu \mathrm{g} / \mathrm{mL}$ were used. Limits of detection (LODs) were calculated based on the calibration curve as $3.3 *$ standard deviation / slope; and limits of quantification (LOQs) as $10 *$ standard deviation / slope.

\section{Data analysis}

Data processing was performed using the MS workstation V.7.0.2 (Agilent technologies). The statistical software program R V.3.6.0 and Microsoft Excel were used to determine flavoring detection frequency and prevalence, the mean number of (unique) flavorings per e-liquid, and median concentrations (including range). Detection frequency is defined as the number of e-liquids in which a flavoring was detected; flavoring prevalence is defined as the number of e-liquids with the flavoring as percentage of the total number of e-liquids (overall or within a category). A heat map (combined with hierarchical clustering) was created to visualize flavoring prevalence. Flavorings and flavor categories were grouped together by similarity in a dendrogram. Clusters of flavor categories were distinguished by cutting off the dendrogram halfway, in order to capture more than $50 \%$ of the variation between the flavor categories. Relative prevalence was used to account for differences in sample size (i.e., number of e-liquids) between the flavor categories. ANOVA and $t$-tests were used to determine differences in the mean number of flavorings per e-liquid between categories. To correct for multiple testing, Benjamini-Hochberg false discovery rate (FDR) ${ }^{26}$ adjusted $p$-values of $<5 \%$ were considered significant. Concentrations of the flavorings in the duplicate runs were averaged for further analyses. 


\section{Results and discussion}

\section{Method validation}

Regarding specificity of the method, none of the flavorings had both the same quantifier ion and a difference in retention time of $<0.4$ minutes (see Appendix Table A5.1). However, the mass spectra of decanal and L-menthol overlapped for a large part and the retention time differed only 0.018 minutes. Therefore, e-liquids that screened positive on either one of those flavorings were manually confirmed. Results for linearity of the method are shown in Appendix Table A5.2. All coefficient of determination $\left(\mathrm{r}^{2}\right)$ values were $>0.993$. RSD response factors were $<10 \%$, except for maltol (25\%) and furaneol (22\%). Repeatability of the retention times and peak areas for the 10 flavorings selected for quantification and the two internal standards is shown in Appendix Table A5.3. Repeatability was generally sufficient, except for ethyl maltol, for which the RSD of the peak area reached $27 \%$. The recovery generally ranged from $92 \%$ to $120 \%$, but was higher for maltol (up to 207\%) (see Appendix Table A5.4). Finally, the LOD and LOQ for each of the 10 flavorings selected for quantification are shown in Appendix Table A5.5. Quantification limits in the e-liquid samples varied between 0.03 (ethyl acetate and ethyl vanillin) and 0.25 (maltol) $\mathrm{mg} / \mathrm{mL}$. In conclusion, validation was considered acceptable, except for ethyl maltol, maltol, and furaneol. Hence, concentrations for these compounds should be considered with appropriate caution.

\section{Most common e-liquid flavorings}

Of the 79 flavorings, 66 were detected in at least one e-liquid. Eighteen flavorings were identified in more than $10 \%$ of the overall sample of e-liquids (see Table 5.2), a cut-off that is consistent with our previous study on e-liquid flavorings ${ }^{10}$. The most frequently identified flavorings were vanillin (present in $42 \%$ of the total set), ethyl butyrate (41\%), and cis-3-hexenol (35\%); their flavors are described as respectively creamy/vanilla, ethereal/fruity, and fresh/green ${ }^{25}$. Of the 320 e-liquids, we detected vanillin, ethyl vanillin, or both in 144 e-liquids (45\%). This is comparable to a previous study in which vanillin and/or ethyl vanillin were identified in approximately half of the e-liquid samples ${ }^{21}$.

Strikingly, most of the flavoring compounds listed in Table 5.2 impart a sweet and/or fruity aroma. This finding directly adds to the ongoing debate in the United States (US) about why teenagers and young adults who did not previously smoke combustible cigarettes started vaping. Previous research showed that young people typically have a preference for sweet taste ${ }^{27}$ and non-smokers are mainly interested in trying sweet e-liquids ${ }^{28}$. This, together with our current and previous findings that sweet and fruity flavoring ingredients dominate ${ }^{10}$ and the e-liquid market is generally dominated by sweet flavors ${ }^{4}$, may provide directions for regulation of (sweet) e-liquid flavors and/or flavorings in order to reduce e-cigarette appeal among youth non-smokers.

Detection frequencies of the top 18 most frequently identified flavorings in this study were comparable to those found in our previous study (Table 5.2, final column), in which we analyzed the Dutch e-liquid market ( $\mathrm{n}=16839$ e-liquids) using information from manufacturers about their e-liquids' flavorings compositions ${ }^{10}$. In addition, other studies using e-liquids from the US ${ }^{23}$ and Germany ${ }^{20}$ reported the same compounds as most prevalent. It would be interesting to 
further investigate why these flavorings in particular are so common, and to perform a comparison between e-liquid (flavoring) compositions on an international level.

Table 5.2: The top 18 most frequently identified flavorings in a sample of 320 e-liquids using GC-MS.

\begin{tabular}{|c|c|c|c|c|}
\hline & Flavoring ingredient & Prevalence & Flavor description & $\begin{array}{l}\text { Prevalence across } \\
16839 \text { e-liquids } \\
\text { reported in the } \\
\text { EU-CEG system* }\end{array}$ \\
\hline 1 & Vanillin & $42 \%$ & Sweet, powerful, creamy, vanilla-like & $35 \%$ \\
\hline 2 & Ethyl butyrate & $41 \%$ & $\begin{array}{l}\text { Ethereal, fruity with buttery-pineapple- } \\
\text { banana, ripe fruit \& juicy notes }\end{array}$ & $28 \%$ \\
\hline 3 & Cis-3-hexenol & $35 \%$ & Strong, fresh, green, grassy & $18 \%$ \\
\hline 4 & Benzyl alcohol & $32 \%$ & $\begin{array}{l}\text { Faint, sweet, almond fruity, somewhat } \\
\text { chemical }\end{array}$ & $14 \%$ \\
\hline 5 & Ethyl maltol & $31 \%$ & Sweet, fruity-caramellic, cotton candy & $32 \%$ \\
\hline 6 & Ethyl vanillin & $25 \%$ & Intense, sweet, creamy, vanilla-like & $19 \%$ \\
\hline 7 & $\gamma$-Decalactone & $23 \%$ & Coconut-peach & $18 \%$ \\
\hline 8 & Methyl cyclopentenolone & $23 \%$ & Very strong, caramellic-maple, lovage & $18 \%$ \\
\hline 9 & Ethyl methyl butyrate & $22 \%$ & $\begin{array}{l}\text { Strong, green, fruity, apple with } \\
\text { strawberry notes }\end{array}$ & $16 \%$ \\
\hline 10 & Isoamyl alcohol & $20 \%$ & $\begin{array}{l}\text { Breathtaking, alcoholic odor; in dilution } \\
\text { a winey-brandy taste }\end{array}$ & $4 \%$ \\
\hline 11 & $\gamma$-Nonalactone & $19 \%$ & Strong, fatty, coconut odor and taste & $10 \%$ \\
\hline 12 & Menthol & $18 \%$ & $\begin{array}{l}\text { Strong trigeminal cooling sensation } \\
\text { with a slight mint note }\end{array}$ & $12 \%$ \\
\hline 13 & Isoamyl isovalerate & $16 \%$ & $\begin{array}{l}\text { Fruity, green-apple, pineapple, tropical, } \\
\text { mango, apricot, cognac }\end{array}$ & $11 \%$ \\
\hline 14 & Ethyl propionate & $15 \%$ & Strong, ethereal, fruity, rum-like & $11 \%$ \\
\hline 15 & Linalool & $15 \%$ & $\begin{array}{l}\text { Sweet floral-woody with slight citrus } \\
\text { notes }\end{array}$ & $15 \%$ \\
\hline 16 & $\gamma$-Octalactone & $13 \%$ & $\begin{array}{l}\text { Sweet-coumarinic, coconut-like odor } \\
\text { and taste }\end{array}$ & $7 \%$ \\
\hline 17 & Cis-3-hexenyl acetate & $12 \%$ & $\begin{array}{l}\text { Strong, fruity-grassy-green odor with } \\
\text { banana notes }\end{array}$ & $9 \%$ \\
\hline 18 & Maltol & $11 \%$ & $\begin{array}{l}\text { Sweet, fruity, berry, strawberry, } \\
\text { caramellic }\end{array}$ & $23 \%$ \\
\hline
\end{tabular}

Prevalence is reported as the number of e-liquids containing the respective flavoring as percentage of the total number of e-liquids. Flavor descriptions were retrieved from a commercial flavor database ${ }^{25}$.

* In a previous study, we analyzed information from manufacturers in the EU-CEG system about flavoring compositions of 16839 e-liquids reported to be marketed in the Netherlands in $2017^{10}$.

EU-CEG, European Common Entry Gate. 


\section{Comparison between flavor categories}

Prevalence of flavorings within a category

The prevalence of the flavorings in total and within each individual flavor category can be found in Appendix Table A5.6. For example, considering the flavorings, vanillin was detected in more than three-quarter of the e-liquids classified as dessert (detection frequency $82 \%$ ), other sweets (77\%), and nuts (75\%), and approximately half of the e-liquids classified as fruit-berries (50\%), coffee/tea (47\%), and other beverages (46\%), but not in any of the e-liquids classified as menthol/ mint, spices, and, as expected, unflavored. As an example regarding the flavor categories, the most frequently detected flavorings within the menthol/mint category were menthol (detected in $75 \%$ of the menthol/mint e-liquids), menthyl acetate (63\%), limonene $(63 \%)$, and menthone $(63 \%)$. All of these compounds are described as having a minty, fresh flavor ${ }^{25}$.

Using the data from Appendix TableA5.6, we visualized the prevalence of flavorings (rows) in e-liquids from the different flavor categories (columns), including a hierarchical clustering of flavorings and flavor categories by similarity (see Figure 5.1). Cutting off the dendrogram halfway resulted in four clusters of flavor categories with similar flavoring prevalence: (1) fruit, candy, alcohol, beverages; (2) dessert, coffee/tea, nuts, sweets; (3) menthol/mint; and (4) spices, tobacco, and unflavored. Compared to other clusters, the first cluster is characterized mostly by a high prevalence of ethyl butyrate (ethereal, fruity flavor), cis-3-hexenol (fresh, green flavor), $\gamma$-decalactone (coconut-peach flavor), and isoamyl alcohol (alcoholic, winey-brandy flavor). This explains why this cluster contains the categories that have particularly artificial/fresh sweet and ethereal e-liquid flavors: fresh fruits, artificial sweet candies such as gummy bears, ethereal alcoholic drinks varying from sweet cocktails to liquors, and sweet fresh beverages such as lemonade and cola. The second cluster can be described by a relatively high prevalence of vanillin and ethyl vanillin (both having a creamy, vanilla flavor), ethyl maltol (sweet, fruitycaramellic flavor), and methyl cyclopentenolone (strong, caramellic-maple flavor). This explains the presence of e-liquids with a particularly "warm" sweet flavor within this cluster: dessert flavors varying from cheesecake to custard, sweet e-liquid flavor descriptions such as vanilla, caramel, and chocolate, coffee flavors varying from cappuccino to caffè mocha, and nut flavors such as peanut with caramel and chocolate. The third cluster contains the menthol/mint category and is clearly characterized by a high prevalence of flavorings described as causing a minty, fresh cooling sensation: menthol, menthyl acetate, limonene, and menthone. The fourth cluster includes e-liquids from typically non-sweet categories: spices, tobacco, and unflavored. These can be described by an overall low flavoring prevalence, particularly regarding the flavorings that characterize the other clusters. Compared to other clusters, prevalence of anethole and p-anisaldehyde (both having an anisic herbaceous flavor), eugenol (clove flavor), and transcinnamaldehyde (cinnamon flavor) are relatively high, which relates to the nature of particular e-liquids from the spice category. Overall, this shows that, as expected, chemical-analytical data on e-liquid flavoring compositions provides information about an e-liquid's flavor label. This is particularly relevant in cases of absent or ambiguous brand names and product descriptions, or when information about e-liquids' flavoring content may not be reliable (e.g., information submitted by manufacturers may not always be complete or correct). 


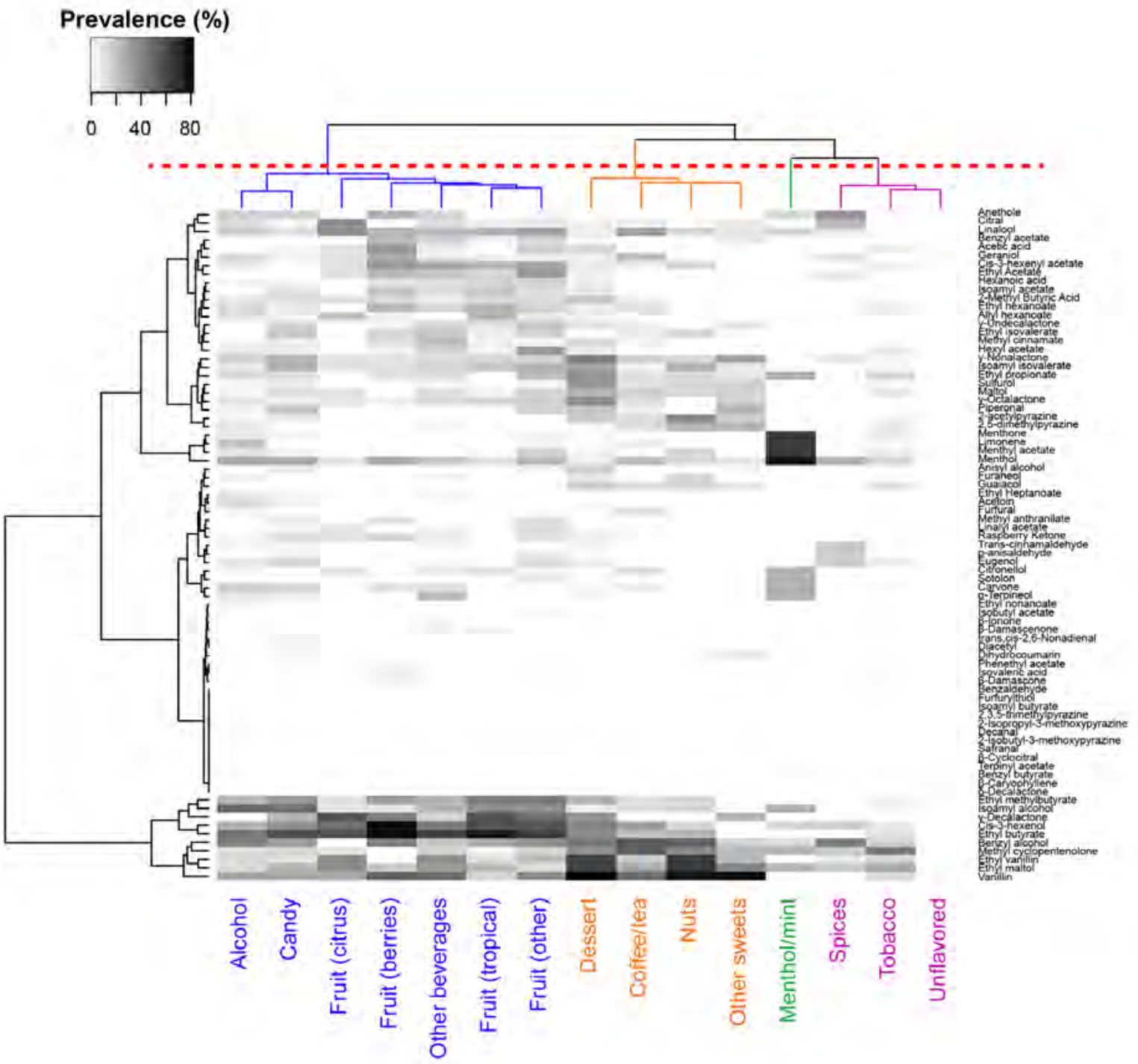

Figure 5.1: Visualization of the prevalence of 79 target flavorings (rows) in e-liquids, for each of the 15 flavor categories (columns). Relative prevalence is expressed on a scale from black to white, which indicates high to low prevalence (\%), respectively. Flavorings and flavor categories are hierarchically clustered (organized as a dendrogram) based on similar data. Cutting off the dendrogram halfway (dotted red line) distinguishes four clusters of flavor categories (highlighted in blue, orange, green, and purple) that represent groups of similar flavoring prevalence data. 
Flavorings detected in any e-liquid within a category

From the 79 target flavorings, zero flavorings were identified in any e-liquid from the unflavored category. Apart from that, the number of target flavorings (79 in total) detected in any (at least one) e-liquid within a category ranged from 15 for the spices category to 52 for the dessert category (see Table 5.3). The number of flavorings detected in more than $10 \%$ of the e-liquids within a category ranged from 7 for tobacco to 34 for fruit-other (Table 3, final column). For most categories, this number was much lower than the number of target flavorings detected in any e-liquid. Thus, part of the flavorings are relatively common for a category, but various flavorings are added to only a few e-liquids within that category. These flavorings are probably used to define a particular e-liquid flavor (i.e., a subcategory of the flavor wheel) or a particular brand.

Table 5.3: The sum of unique target flavorings (79 in total) detected in any (i.e., at least one) e-liquid within a category, and in more than $10 \%$ of the e-liquids within a category.

\begin{tabular}{lll}
\hline Flavor category & $\begin{array}{l}\text { Sum of unique flavorings } \\
(\mathbf{n}=\mathbf{7 9})\end{array}$ & $\begin{array}{l}\text { Sum of unique flavorings }(\mathbf{n}=\mathbf{7 9}) \\
\text { in }>\mathbf{1 0 \%} \text { of the e-liquids }\end{array}$ \\
\hline Dessert & 52 & 22 \\
Candy & 49 & 25 \\
Fruit (other) & 48 & 34 \\
Other beverages & 45 & 31 \\
Alcohol & 44 & 20 \\
Fruit (berries) & 35 & 20 \\
Coffee/tea & 33 & 23 \\
Fruit (tropical) & 32 & 19 \\
Tobacco & 29 & 7 \\
Fruit (citrus) & 25 & 9 \\
Nuts & 23 & 23 \\
Other sweets & 22 & 13 \\
Menthol/mint & 16 & 16 \\
Spices & 15 & 10 \\
Unflavored & 0 & 0 \\
\hline
\end{tabular}




\section{Mean number of flavorings per e-liquid}

In total, 1969 flavorings were detected in 320 e-liquids, of which two e-liquids were marketed as unflavored. In 14 e-liquids that were marketed as having a flavor (4\% of total sample) and therefore expected to contain flavorings, zero of the target flavorings were detected. As we used a targeted approach rather than an open screening approach, flavorings outside the target list were not identified, and thus, unknown compounds may be present.

The mean number ( \pm standard deviation) of flavorings per individual e-liquid was 6 \pm 4 for the overall sample. The mean number of flavorings per e-liquid was zero within the unflavored category, and further ranged from $3 \pm 3$ for e-liquids classified as tobacco to $8 \pm 4$ for both dessert and fruit-other (see Figure 5.2).

We found significant differences in the mean number of flavorings per e-liquid between categories $(p=3.72 \mathrm{E}-11)$. The mean number of flavorings per e-liquid within the alcohol, other beverages, fruit-berries, fruit-tropical, fruit-other, dessert, and candy categories were significantly higher than within the tobacco, spices, and unflavored categories $(p<0.05)$. This is consistent with the low number of flavorings per e-liquid as a potential reason for hierarchical clustering of the tobacco, spices, and unflavored categories based on flavoring prevalence (see section Prevalence of flavorings within a category). The mean number of flavorings per e-liquid for dessert and fruit-other was also significantly higher than for the plausibly related other sweets and fruit-citrus categories $(p<0.05)$. This can be explained by the type of flavors within these categories: the other sweets and fruit-citrus categories contains e-liquids with a relatively simple flavor such as vanilla, caramel, or lemon; flavors that could be created with only a few flavorings (e.g., vanillin for vanilla flavor and limonene for citrus flavor). In contrast, the dessert and fruitother categories contain many e-liquids with multiple flavor descriptors, for example respectively blueberry cheesecake and a pomegranate-flavored e-liquid with hints of anise, violet, and mint. Thus, our results show that e-liquids with a combined flavor description contain more different flavorings than e-liquids described as simply having one flavor. See Appendix Table A5.7 for other significant differences and $p$-values. 


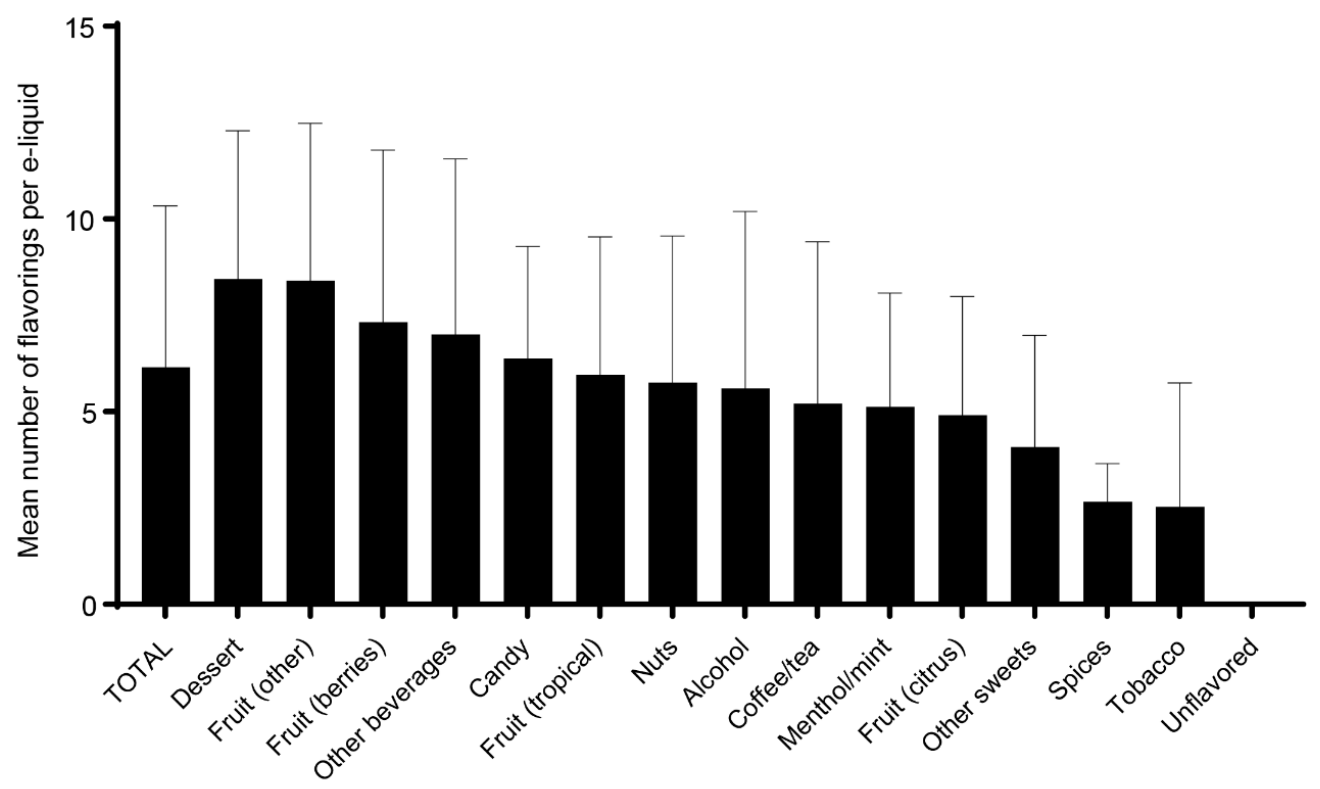

Figure 5.2: Mean $( \pm \mathrm{SD})$ number of flavorings per e-liquid for the total dataset $(n=320$ e-liquids) and for each individual flavor category. The mean number of flavorings per e-liquids was significantly lower for the unflavored category compared to all other categories, except for menthol/mint and nuts. The mean number of flavorings per e-liquids was significantly lower for both tobacco and spice compared to alcohol, other beverages, fruit (berries, tropical, and other), dessert, and candy. See Appendix Table A5.7 for $p$-values.

\section{Flavorings with a low detection frequency}

Some flavorings that manufacturers reported to have added to more than $10 \%$ of the e-liquids on the Dutch market (see previous study ${ }^{10}$ ) were identified in this study with a frequency of less than $5 \%$ or not at all: furaneol (identified in $2.5 \%$ of the e-liquids included in this study), benzyl butyrate $(0 \%)$, benzaldehyde $(0 \%), \delta$-decalactone $(0 \%)$. The difference in detection frequency between both studies may be assigned to the different study aims, and thereby the selection of e-liquid flavors. That is, in the current study, we selected only a maximum of two e-liquids per flavor subcategory in order to obtain an optimal representation of each main category, while our previous study included the complete set of e-liquids and their flavors as reported to be marketed in the Netherlands, thereby containing much more e-liquids within some flavor subcategories.

Other reasons why some flavorings that manufacturers reported to have added were not at all detected in the current study may be that their concentrations were below our limit of detection, or due to the chemically unstable character of the e-liquid. For example, benzaldehyde and other flavoring aldehydes have been shown to rapidly react with the e-liquid solvent propylene glycol (PG), which causes almost half of the aldehyde content to be converted into flavor aldehyde PG acetals ${ }^{11}$. Thus, even though manufacturers reported to add flavoring aldehydes to e-liquids, chemical analysis of the neat e-liquid may show distinct chemical profiles due to a changed 
composition during storage. Flavoring aldehyde profiles may also differ between the neat e-liquid and the e-cigarette aerosol ${ }^{16}$, as aldehydes are formed during the aerosolization process by pyrolysis of $\mathrm{PG}$ and $\mathrm{VG}^{29}$. More chemical-analytical research is needed to obtain insight in the part of flavoring aldehydes, as well as other potentially reactive compounds, that is added, converted, and formed, when chemically analyzing the e-liquid itself compared to e-cigarette aerosol. Also, not only regarding the aldehyde content, further research is needed to chemically assess the complete composition of e-cigarette aerosol and the associated health risks. Although this type of research provides insight in what users are actually exposed to, it will be extremely time-consuming.

\section{Flavoring concentrations}

The median concentrations and ranges of the 10 flavorings quantified, stratified by flavor category, are shown in Appendix Table A5.8. Within the flavor categories, concentrations of some flavorings varied substantially. For example, within the dessert category, vanillin concentrations ( $\mathrm{n}=50$ data points) ranged from $0.4-13.5 \mathrm{mg} / \mathrm{mL}$, ethyl vanillin concentrations (37 data points) ranged from $0.2-12.8 \mathrm{mg} / \mathrm{mL}$, and ethyl maltol concentrations (28 data points) ranged from $<0.1-17.3 \mathrm{mg} / \mathrm{mL}$. No statistical comparisons of flavoring concentrations between categories could be performed, as the number of data points within a category was often too low or sometimes even zero. In order to determine whether manufacturers create different e-liquid flavors by varying flavoring concentrations besides adding distinct flavorings, further research is needed. For example, flavoring concentrations could be statistically compared between flavor categories, which requires a large number of data points (i.e., sufficient e-liquids containing the respective flavoring to perform such analyses). Also, as most e-liquids contain multiple flavorings, future research is needed not only to investigate the relation between concentrations and perception of individual flavorings, but also the interaction between the flavoring mixtures, and how differences in concentrations may influence overall flavor perception ${ }^{30}$.

\section{Identification of nicotine}

The nicotine content as stated on the e-liquid package varied from $0-18 \mathrm{mg} / \mathrm{mL}$. We identified nicotine in 16 e-liquids that were marketed as nicotine-free (5\% of total sample). In 3 e-liquids that were marketed as having $6 \mathrm{mg} / \mathrm{mL}$ nicotine, nicotine was not identified. This is in line with a previous study showing nicotine labelling discrepancies ${ }^{20}$, and supports our hypothesis that information from manufacturers may not always be correct, thereby confirming the importance of chemical-analytical measurements.

\section{Limitations}

A few limitations of this study should be noted. Firstly, it is hard to separate stereoisomers, but many flavorings are chiral and the odor of two isomers may differ (e.g., R-carvone has a spearmint odor and S-carvone has a spicy, caraway odor). Furthermore, the validation experiment did not include flavorings with close structural similarities to our target flavorings (e.g., alpha- and beta-damascone, which differ only in the position of a double bond). Therefore, conclusive identification of these types of flavorings in e-liquids would require additional analyses. Secondly, only positively identified flavorings were confirmed using standards, and 
quantification of the 10 flavorings selected was only performed in e-liquids where the respective flavoring was positively identified. This means that false negatives may have remained, which were not included in the quantification analysis and may have caused an underestimation of flavoring prevalence. Thirdly, analyzing the standards of maltol, ethyl maltol, and furaneol using GC-MS resulted in tailing peaks, explaining the suboptimal recovery and relatively high RSD response factors for these compounds. Although validation results for the other compounds were considered acceptable, determined concentrations of maltol, ethyl maltol, and furaneol should be treated with appropriate caution. Peaks of PG and VG also tended to be tailed and their spectra may show some overlap with spectra of the target flavorings. Although identification of the flavorings was based on the reference spectra of individual standards, PG and VG may have affected our quantification results. To minimize the carryover effect between samples, a blanc sample with ethanol was run between each test sample.

It should also be noted that classification of the e-liquids into the main categories of the flavor wheel was based on the e-liquids' flavor as a whole or the first flavor descriptor mentioned 24; other flavor descriptors were not taken into account in this study. Including more than one flavor descriptor would result in some e-liquids being classified in multiple flavor categories. Although this would be a more accurate approach to flavor classification, it is not possible, within an e-liquid, to separate the flavorings that contribute to the e-liquid's main flavor category from the flavorings that contribute to the secondary flavor descriptors.

Further, the resulting clusters of the flavor categories were based on the prevalence of the flavorings tested in this study. Selecting different target compounds or cutting off the dendrogram at a higher or lower level may result in different clusters of flavor categories. However, as the prevalence results were comparable to those reported by the industry and found in previous chemical-analytical studies, we believe that the majority of flavorings important for distinguishing between e-liquid flavor categories were covered in our analysis.

Finally, we quantified the flavorings that were most prevalent according to information from manufacturers as analyzed in our previous study. However, these compounds were not necessarily most prevalent according to our current GC-MS results. As some of the 10 flavorings selected for quantification were not detected in each flavor category or only in a few e-liquids, groups were too small to statistically compare concentrations between the 15 flavor categories. 


\section{Conclusions}

This study used a simple and pragmatic GC-MS method to identify and quantify target flavorings in a large sample of e-liquids with different marketed flavor descriptions. Vanillin (creamy/ vanilla flavor), ethyl butyrate (ethereal/fruity flavor), and cis-3-hexenol (fresh/green flavor) were most frequently detected in the overall dataset. Based on similarities in flavoring prevalence, four clusters of flavor categories could be distinguished: (1) fruit, candy, alcohol, beverages; (2) dessert, coffee/tea, nuts, sweets; (3) menthol/mint; and (4) spices, tobacco, and unflavored. These clusters can be characterized by the presence or absence of particular flavorings, and by the mean number of flavorings per e-liquid. This shows that, as expected, chemical-analytical data on e-liquid flavoring compositions provides information about an e-liquid's flavor label, and that e-liquids from some flavor categories are more similar in terms of flavoring compositions than others. This information could be used, for example, when product descriptions are absent or ambiguous, or when regulators have no access to information from manufacturers about e-liquids' flavoring content. In addition, our study showed that flavoring concentrations varied within the overall dataset. Additional research is needed to compare flavoring concentrations between the individual flavor categories in order to investigate whether manufacturers create different types of e-liquid flavors by varying flavoring concentrations besides adding distinct compounds. In conclusion, our comparison of flavoring compositions between e-liquid flavor categories may provide focus to regulators, sensory scientists, and toxicologists in their efforts to respectively decrease e-liquid appeal for particularly youth, and to further investigate e-liquid appeal and the potentially harmful effects of inhaling particular e-liquid constituents such as flavorings. 


\section{Appendix}

Appendix Table A5.1: Detection parameters (i.e., specificity) of the 79 target flavorings, nicotine, and the two internal standards (benzene-d6 and n-heptadecane).

\begin{tabular}{|c|c|c|c|c|c|}
\hline$\#$ & $\begin{array}{l}\text { RT } \\
(\mathrm{min})\end{array}$ & $\begin{array}{l}\text { Chemical } \\
\text { compound }\end{array}$ & $\begin{array}{l}\text { Quantifier } \\
\text { ion }(\mathrm{m} / \mathrm{z})\end{array}$ & $\begin{array}{l}\text { Reference } \\
\text { spectrum }(\mathrm{m} / \mathrm{z})\end{array}$ & Flavor description \\
\hline 1 & 10.279 & Acetic acid & 43.0 & $43.0,45.0,60.0$ & Pungent, sour, acid, vinegar \\
\hline 2 & 11.368 & Diacetyl & 43.0 & $43.0,42.0,43.9$ & Strong, buttery \\
\hline 3 & 11.765 & Ethyl acetate & 43.0 & $32.0,45.0,43.0$ & Ethereal, sharp, wine-brandy-like \\
\hline 4 & 13.324 & Benzene-d6 & 84.1 & $84.1,82.1,56.0$ & NA (internal standard) \\
\hline 5 & 14.571 & Ethyl propionate & 57.0 & $57.0,74.1,29.0$ & Strong, ethereal, fruity, rum-like \\
\hline 6 & 15.231 & Isoamyl alcohol & 55.1 & $55.1,70.1,41.1$ & $\begin{array}{l}\text { Breathtaking, alcoholic odor; in } \\
\text { dilution a winey-brandy taste }\end{array}$ \\
\hline 7 & 15.494 & Acetoin & 89.0 & $89.0,43.0,45.0$ & Creamy-buttery, yogurt-like \\
\hline 8 & 16.135 & Isobutyl acetate & 43.0 & $43.0,56.0,41.0$ & Fruity, banana-apple-pear-pineapple \\
\hline 9 & 16.852 & Ethyl butyrate & 71.0 & $71.1,88.0,43.1$ & $\begin{array}{l}\text { Ethereal, fruity with buttery-pineapple- } \\
\text { banana, ripe fruit \& juicy notes }\end{array}$ \\
\hline 10 & 17.312 & Isovaleric acid & 60.0 & $60.0,41.0,87.0$ & $\begin{array}{l}\text { Very sour,"sweaty", cheesy, odor; } \\
\text { fruity on dilution }\end{array}$ \\
\hline 11 & 17.555 & $\begin{array}{l}\text { 2-Methyl butyric } \\
\text { acid }\end{array}$ & 74.0 & $74.0,41.0,57.0$ & $\begin{array}{l}\text { Pungent, acrid, Roquefort cheese } \\
\text { flavor; fruity in dilution }\end{array}$ \\
\hline 12 & 17.927 & $\begin{array}{l}\text { Ethyl methyl } \\
\text { butyrate }\end{array}$ & 102.0 & $57.1,102.1,41.0$ & $\begin{array}{l}\text { Strong, green, fruity, apple with } \\
\text { strawberry notes }\end{array}$ \\
\hline 13 & 17.989 & Ethyl isovalerate & 88.0 & $88.0,57.1,85.1$ & $\begin{array}{l}\text { Strong, fruity apple flavor with } \\
\text { buttery-wine-banana-blueberry notes }\end{array}$ \\
\hline 14 & 18.487 & Cis-3-hexenol & 67.1 & $67.2,41.0,82.1$ & Strong, fresh, green, grassy \\
\hline 15 & 18.491 & Isoamyl acetate & 42.9 & $67.1,55.1,163.0$ & Sweet, fruity, banana, pear \\
\hline 16 & 19.119 & Furfural & 95.1 & $95.1,96.0,39.0$ & $\begin{array}{l}\text { Sweet, cereal, bread-like, yeasty, } \\
\text { caramellic, spicy almond notes }\end{array}$ \\
\hline 17 & 20.365 & $\begin{array}{l}\text { 2,5-Dimethyl } \\
\text { pyrazine }\end{array}$ & 108.1 & $108.1,42.0,39.0$ & Chocolate, roasted nuts, earthy \\
\hline 18 & 20.454 & Hexanoic acid & 60.0 & $60.0,73.0,41.0$ & Heavy, fatty, cheesey-sweaty \\
\hline 19 & 20.542 & Furfurylthiol & 81.0 & $81.0,53.1,114.0$ & $\begin{array}{l}\text { Powerful burnt, coffee-like, caramellic } \\
\& \text { alliaceous on dilution }\end{array}$ \\
\hline 20 & 21.053 & Ethyl hexanoate & 88.0 & $88.0,43.0,99.1$ & $\begin{array}{l}\text { Strong, fruity, pineapple, banana; } \\
\text { strawberry, pear \& tropical notes }\end{array}$ \\
\hline 21 & 21.329 & Hexyl acetate & 43.0 & $43.0,56.1,61.0$ & Sweet, fruity, green, pear-apple-like \\
\hline 22 & 21.383 & $\begin{array}{l}\text { Cis-3-hexenyl } \\
\text { acetate }\end{array}$ & 67.1 & $67.1,43.0,82.1$ & $\begin{array}{l}\text { Strong, fruity-grassy-green odor with } \\
\text { banana notes }\end{array}$ \\
\hline 23 & 21.884 & D-Limonene & 67.0 & $67.1,93.1,68.1$ & $\begin{array}{l}\text { Fresh, sweet, hydrocarbon and orange } \\
\text { citrus odor }\end{array}$ \\
\hline 24 & 22.038 & Benzaldehyde & 77.0 & $77.0,105.1,106.0$ & $\begin{array}{l}\text { Odor of bitter almond oil; } \\
\text { characteristic sweet cherry taste }\end{array}$ \\
\hline 25 & 22.045 & Isoamyl butyrate & 71.0 & $70.1,71.1,43.0$ & $\begin{array}{l}\text { Mixed fruit odor; sweet, apricot-apple- } \\
\text { banana-pear flavor }\end{array}$ \\
\hline 26 & 22.162 & $\begin{array}{l}\text { 2,3,5-Trimethyl } \\
\text { pyrazine }\end{array}$ & 122.1 & $122.1,42.0,39.0$ & $\begin{array}{l}\text { Baked potato, roasted nut, cocoa, } \\
\text { coffee, burnt }\end{array}$ \\
\hline
\end{tabular}




\begin{tabular}{|c|c|c|c|c|c|}
\hline \# & $\begin{array}{l}\text { RT } \\
(\min )\end{array}$ & $\begin{array}{l}\text { Chemical } \\
\text { compound }\end{array}$ & $\begin{array}{l}\text { Quantifier } \\
\text { ion }(\mathrm{m} / \mathrm{z})\end{array}$ & $\begin{array}{l}\text { Reference } \\
\text { spectrum }(\mathrm{m} / \mathrm{z})\end{array}$ & Flavor description \\
\hline 27 & 22.640 & Allyl hexanoate & 99.1 & $99.1,43.0,71.1$ & Fatty, fruity, winey-pineapple-like \\
\hline 28 & 22.810 & Ethyl heptanoate & 88.1 & $88.1,43.0,55.1$ & Strong, fruity, winey, cognac-like \\
\hline 29 & 22.834 & Isoamyl isovalerate & 70.1 & $70.1,85.0,57.0$ & $\begin{array}{l}\text { Fruity, green-apple, pineapple, tropical, } \\
\text { mango, apricot, cognac }\end{array}$ \\
\hline 30 & 23.037 & 2-Acetylpyrazine & 94.1 & $94.1,52.0,80.1$ & $\begin{array}{l}\text { Characteristic of popcorn; nutty, bread } \\
\text { cru }\end{array}$ \\
\hline 31 & 23.084 & Linalool & 93.0 & $93.1,71.1,55.0$ & $\begin{array}{l}\text { Sweet floral-woody with slight citrus } \\
\text { notes }\end{array}$ \\
\hline 32 & 23.122 & $\begin{array}{l}\text { Methyl } \\
\text { cyclopentenolone }\end{array}$ & 112.0 & $112.0,55.1,69.1$ & Very strong, caramellic-maple, lovage \\
\hline 33 & 23.210 & Benzyl alcohol & 79.1 & $79.1,77.1,108.1$ & $\begin{array}{l}\text { Faint, sweet, almond fruity, somewhat } \\
\text { chemical }\end{array}$ \\
\hline 34 & 23.225 & Furaneol & 43.0 & $43.0,128.1,57.0$ & $\begin{array}{l}\text { Fruity, caramelized pineapple- } \\
\text { strawberry; roasted }\end{array}$ \\
\hline 35 & 23.378 & $\begin{array}{l}\text { 2-Isopropyl-3- } \\
\text { methoxypyrazine }\end{array}$ & 137.2 & $\begin{array}{l}137.2,124.2 \\
152.1\end{array}$ & $\begin{array}{l}\text { Green peas, earthy, bell pepper, raw } \\
\text { potato, and galbanum }\end{array}$ \\
\hline 36 & 24.165 & Guaiacol & 81.1 & $81.1,109.1,124.1$ & $\begin{array}{l}\text { Strong, sweet, smoke-like \& vanilla, } \\
\text { phenolic-medicinal notes }\end{array}$ \\
\hline 37 & 24.443 & $\begin{array}{l}\text { Sotolon (synoym: } \\
\text { 4,5-dimethyl- } \\
\text { 3-hydroxy-2,5- } \\
\text { dihydrofuran-) }\end{array}$ & 83.1 & $83.1,129.0,84.0$ & $\begin{array}{l}\text { Powerful caramel aroma; sweet burnt } \\
\text { taste }\end{array}$ \\
\hline 38 & 24.571 & $\begin{array}{l}\text { trans,cis-2,6- } \\
\text { Nonadienal }\end{array}$ & 70.1 & $70.1,69.1,41.0$ & $\begin{array}{l}\text { Cucumber-like, green melon or violet } \\
\text { leaf }\end{array}$ \\
\hline 39 & 24.851 & Menthone & 112.0 & $69.1,112.1,55.0$ & $\begin{array}{l}\text { Minty-herbaceous (not green); dry } \\
\text { woody notes }\end{array}$ \\
\hline 40 & 24.879 & L-menthol & 81.1 & $81.1,95.1,71.1$ & $\begin{array}{l}\text { Strong trigeminal cooling sensation } \\
\text { with a slight mint note }\end{array}$ \\
\hline 41 & 24.897 & Decanal & 57.0 & $57.1,41.0,55.1$ & $\begin{array}{l}\text { Strong, penetrating, sweet, waxy, } \\
\text { orange peel odor; citrus taste }\end{array}$ \\
\hline 42 & 24.897 & $\begin{array}{l}\text { 2-Isobutyl-3- } \\
\text { methoxypyrazine }\end{array}$ & 124.1 & $124.1,81.1,94.1$ & Characteristic Green Bell Pepper \\
\hline 43 & 25.005 & Maltol & 126.0 & $126.1,71.1,97.1$ & $\begin{array}{l}\text { Sweet, fruity, berry, strawberry, } \\
\text { caramellic }\end{array}$ \\
\hline 44 & 25.209 & Benzyl acetate & 108.0 & $108.1,91.1,79.1$ & $\begin{array}{l}\text { Sweet, floral, fruity odor of jasmin and } \\
\text { gardenia }\end{array}$ \\
\hline 45 & 25.294 & Citronellol & 67.1 & $67.1,69.1,81.1$ & Rose-like \\
\hline 46 & 25.383 & $\alpha$-Terpineol & 59.0 & $59.0,93.0,81.1$ & Very sweet, floral (lilac), lime \\
\hline 47 & 25.499 & Linalyl acetate & 93.1 & $93.1,80.1,43.0$ & $\begin{array}{l}\text { Sweet, floral, fruity odor; citrus-pear } \\
\text { notes }\end{array}$ \\
\hline 48 & 25.913 & Geraniol & 69.1 & $69.1,41.0,67.1$ & $\begin{array}{l}\text { Sweet, floral, rose-like odor; fruity } \\
\text { taste }\end{array}$ \\
\hline 49 & 25.932 & Ethyl nonanoate & 88.1 & $88.1,73.1,55.0$ & $\begin{array}{l}\text { Fatty-waxy, oily, cognac, grape, nut- } \\
\text { like odor; wine-cognac tropical taste }\end{array}$ \\
\hline 50 & 25.985 & Safranal & 91.0 & $91.1,107.1,105.0$ & $\begin{array}{l}\text { Powerful saffron aroma and taste; } \\
\text { tobacco-camphoraceous notes }\end{array}$ \\
\hline 51 & 26.239 & $\beta$-Cyclocitral & 67.1 & $67.1,109.0,81.1$ & $\begin{array}{l}\text { Sweet, mild green, grassy floral hay- } \\
\text { like, minty, slightly fruity odor }\end{array}$ \\
\hline
\end{tabular}




\begin{tabular}{|c|c|c|c|c|c|}
\hline \# & $\begin{array}{l}\text { RT } \\
(\min )\end{array}$ & $\begin{array}{l}\text { Chemical } \\
\text { compound }\end{array}$ & $\begin{array}{l}\text { Quantifier } \\
\text { ion }(\mathrm{m} / \mathrm{z})\end{array}$ & $\begin{array}{l}\text { Reference } \\
\text { spectrum }(\mathrm{m} / \mathrm{z})\end{array}$ & Flavor description \\
\hline 52 & 26.306 & Ethyl maltol & 140.0 & $140.1,139.1,97.1$ & Sweet, fruity-caramellic, cotton candy \\
\hline 53 & 26.494 & Menthyl acetate & 95.0 & $95.1,81.1,43.0$ & $\begin{array}{l}\text { Sweet, fruity-sour, weak, minty } \\
\text { cooling sensation }\end{array}$ \\
\hline 54 & 26.515 & Citral & 69.1 & $69.1,41.0,84.1$ & Strong, lemon-like \\
\hline 55 & 26.680 & Phenethyl acetate & 104.0 & $104.0,43.0,91.1$ & Sweet, rose, fruity, honey \\
\hline 56 & 26.708 & R-carvone & 82.0 & $82.1,54.0,93.1$ & Spearmint odor \& taste \\
\hline 57 & 27.200 & Anethole & 148.2 & $\begin{array}{l}148.2,147.2 \\
117.1\end{array}$ & $\begin{array}{l}\text { Sweet, herbaceous, anise (artificial } \\
\text { Licorice) }\end{array}$ \\
\hline 58 & 27.221 & Y-Octalactone & 85.0 & $85.0,57.0,56.0$ & Sweet-coumarinic, coconut-like \\
\hline 59 & 27.571 & p-Anisaldehyde & 135.1 & $135.1,136.1,77.1$ & $\begin{array}{l}\text { Floral, hay-like odor; sweet anisic- } \\
\text { vanilla-fruity herbaceous }\end{array}$ \\
\hline 60 & 27.631 & Terpinyl acetate & 93.1 & $93.1,121.1,43.0$ & Herbaceous, sweet spicy bergamot \\
\hline 61 & 27.711 & $\begin{array}{l}\text { Trans- } \\
\text { cinnamaldehyde }\end{array}$ & 78.2 & $78.2,131.1,77.2$ & $\begin{array}{l}\text { Spicy, cinnamon-cassia-like with sweet } \\
\text { warm (hot) taste }\end{array}$ \\
\hline 62 & 27.796 & Anisyl alcohol & 109.0 & $\begin{array}{l}109.0,121.1 \\
138.1\end{array}$ & $\begin{array}{l}\text { Sweet, fruity, floral, balsamic anisic- } \\
\text { vanilla-creamy-coumarinic like }\end{array}$ \\
\hline 63 & 27.915 & Sulfurol & 112.0 & $112.0,113.0,85.0$ & $\begin{array}{l}\text { Meaty-beef like with nutty note in } \\
\text { dilution }\end{array}$ \\
\hline 64 & 28.040 & Benzyl butyrate & 91.1 & $91.1,108.1,65.1$ & $\begin{array}{l}\text { Fruity floral, plum, tropical apricot- } \\
\text { pear-like }\end{array}$ \\
\hline 65 & 28.581 & Eugenol & 164.0 & $164.1,91.1,103.1$ & $\begin{array}{l}\text { Strong, spicy, dry, pungent, smoky, } \\
\text { clove-like }\end{array}$ \\
\hline 66 & 28.789 & $\beta$-Damascenone & 69.1 & $69.1,121.1,105.1$ & $\begin{array}{l}\text { Fruity-floral with apple-plum-raisin- } \\
\text { pune, tea, rose, tobacco notes }\end{array}$ \\
\hline 67 & 28.872 & Nicotine & 84.1 & $84.1,133.2,161.1$ & NA \\
\hline 68 & 28.932 & Y-Nonalactone & 85.0 & $85.0,57.0,55.1$ & Strong, fatty, coconut \\
\hline 69 & 29.174 & Methyl anthranilate & 119.0 & $119.0,92.0,151.0$ & Musty, neroli, grape-like \\
\hline 70 & 29.187 & $\beta$-Caryophyllene & 133.1 & $91.0,93.1,79.1$ & $\begin{array}{l}\text { Woody, spicy, dry, with citrus } \\
\text { undernote }\end{array}$ \\
\hline 71 & 29.312 & $\beta$-Damascone & 177.1 & $177.1,81.1,69.1$ & $\begin{array}{l}\text { Complex odour of blackcurrant, plum, } \\
\text { rose, honey and tobacco }\end{array}$ \\
\hline 72 & 29.381 & Methyl cinnamate & 131.0 & $131.0,103.0,77.1$ & $\begin{array}{l}\text { Fruity-balsamic odor; sweet fruity } \\
\text { (cherry-strawberry) taste }\end{array}$ \\
\hline 73 & 29.465 & Piperonal & 149.0 & $\begin{array}{l}149.0,150.0 \\
121.0\end{array}$ & Sweet cherry-vanilla \\
\hline 74 & 30.619 & Vanillin & 151.0 & $151.1,152.0,81.1$ & Sweet, powerful, creamy, vanilla-like \\
\hline 75 & 30.702 & $\beta$-Ionone & 177.1 & $177.1,91.1,43.0$ & Woody, violet, fruity, raspberry \\
\hline 76 & 30.881 & $\gamma$-Decalactone & 85.0 & $195.1,85.1,197.1$ & Coconut-peach \\
\hline 77 & 30.897 & Dihydrocoumarin & 91.1 & $91.1,120.2,148.1$ & Very sweet, nut, hay, coumarin-tobacco \\
\hline 78 & 31.420 & Ethyl vanillin & 137.0 & $\begin{array}{l}137.0,166.1 \\
138.0\end{array}$ & Intense, sweet, creamy, vanilla-like \\
\hline 79 & 31.906 & $\delta$-Decalactone & 99.0 & $99.0,71.1,55.0$ & $\begin{array}{l}\text { Sweet, creamy, buttery, milky, peach, } \\
\text { nut }\end{array}$ \\
\hline 80 & 31.908 & n-Heptadecaan & 57.1 & $57.1,71.1,85.1$ & NA (internal standard) \\
\hline 81 & 33.241 & $\gamma$-Undecalactone & 85.0 & $85.0,55.1,41.0$ & Strong fatty, peach-apricot \\
\hline 82 & 33.878 & Raspberry Ketone & 107.0 & $107.0,164.1,77.1$ & Very sweet, fruity, raspberry \\
\hline
\end{tabular}

Flavor descriptions are based on a commercial flavor database ${ }^{25}$. RT, retention time. 

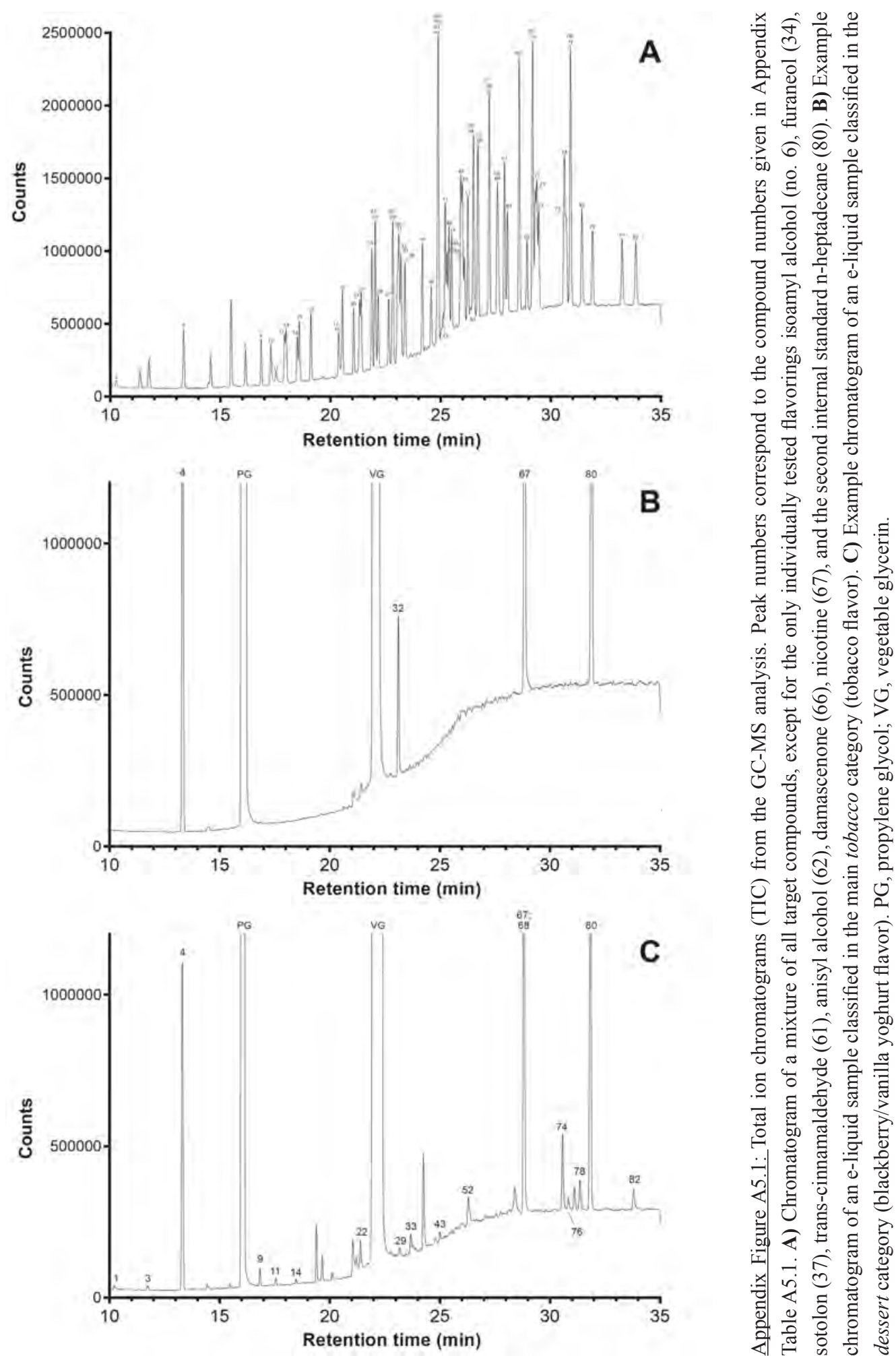
Appendix Table A5.2: Linearity of the 10 calibration curves, determined over 10 concentrations analyzed in independently weighted duplicates, including concentration ranges, coefficients of determination $\left(\mathrm{r}^{2}\right)$, and RSD response factors.

\begin{tabular}{llllll}
\hline Flavoring & $\begin{array}{l}\text { Lower } \\
\text { limit } \\
(\boldsymbol{\mu g} / \mathbf{m L})\end{array}$ & $\begin{array}{l}\text { Upper } \\
\text { limit } \\
(\boldsymbol{\mu g} / \mathbf{m L})\end{array}$ & $\mathbf{r}^{2}$ & $\begin{array}{l}\text { RSD response } \\
\text { factor }(\%)\end{array}$ & Calibration curve \\
\hline Ethyl acetate & 10 & 100 & 0.999 & 2.87 & $\mathrm{y}=0.0053 \mathrm{x}-0.00003$ \\
Cis-3-hexenol & 10 & 100 & 1.000 & 6.79 & $\mathrm{y}=0.0063 \mathrm{x}-0.01250$ \\
Ethyl butyrate & 10 & 100 & 0.999 & 5.69 & $\mathrm{y}=0.0042 \mathrm{x}-0.01260$ \\
Ethyl maltol & 30 & 100 & 0.993 & 6.62 & $\mathrm{y}=0.0036 \mathrm{x}-0.02280$ \\
Ethyl vanillin & 10 & 80 & 0.999 & 3.13 & $\mathrm{y}=0.0152 \mathrm{x}-0.01927$ \\
$\gamma$-Decalactone & 10 & 90 & 0.990 & 9.58 & $\mathrm{y}=0.0103 \mathrm{x}+0.07200$ \\
Maltol & 30 & 90 & 0.996 & 24.73 & $\mathrm{y}=0.0045 \mathrm{x}-0.05530$ \\
Vanillin & 10 & 100 & 0.999 & 4.09 & $\mathrm{y}=0.0085 \mathrm{x}-0.01319$ \\
Methyl cyclopentenolone & 10 & 100 & 0.993 & 5.75 & $\mathrm{y}=0.0030 \mathrm{x}+0.00620$ \\
Furaneol & 10 & 100 & 0.997 & 22.09 & $\mathrm{y}=0.0021 \mathrm{x}-0.01310$ \\
\hline
\end{tabular}

Flavorings were dissolved in the internal standard solution: a solution of the internal standards benzene-d6 and n-heptadecane in ethanol (both $100.0 \mu \mathrm{g} / \mathrm{L}$ ). RSD, relative standard deviation.

Appendix Table A5.3: Repeatability results for the 10 flavorings selected for quantification and the 2 internal standards.

\begin{tabular}{|c|c|c|c|c|c|c|}
\hline & \multicolumn{2}{|c|}{ Retention time } & \multicolumn{4}{|c|}{ Peak area } \\
\hline \multicolumn{3}{|l|}{ PG/VG ratio } & $30 / 70$ & $30 / 70$ & $70 / 30$ & $70 / 30$ \\
\hline \multicolumn{3}{|l|}{ Conc. $(\mu \mathrm{g} / \mathrm{mL})$} & 20 & 80 & 20 & 80 \\
\hline Flavoring & $\begin{array}{c}\text { Mean RT } \\
(\mathrm{n}=24)\end{array}$ & $\begin{array}{c}\text { RSD } \\
(\mathrm{n}=24)\end{array}$ & $\begin{array}{c}\text { RSD } \\
(n=6)\end{array}$ & $\begin{array}{c}\text { RSD } \\
(\mathrm{n}=6)\end{array}$ & $\begin{array}{c}\mathrm{RSD} \\
(\mathrm{n}=6)\end{array}$ & $\begin{array}{c}\text { RSD } \\
(\mathrm{n}=6)\end{array}$ \\
\hline Cis-3-hexenol & 18.48 & $0.03 \%$ & $1.61 \%$ & $1.55 \%$ & $0.77 \%$ & $2.32 \%$ \\
\hline Ethyl acetate & 11.74 & $0.05 \%$ & $1.34 \%$ & $1.32 \%$ & $0.82 \%$ & $2.10 \%$ \\
\hline Ethyl butyrate & 16.85 & $0.04 \%$ & $1.98 \%$ & $1.49 \%$ & $1.77 \%$ & $2.17 \%$ \\
\hline Ethyl maltol & 26.30 & $0.04 \%$ & $27.10 \%$ & $4.07 \%$ & $25.53 \%$ & $4.50 \%$ \\
\hline Ethyl vanillin & 31.40 & $0.02 \%$ & $1.13 \%$ & $1.11 \%$ & $1.44 \%$ & $1.60 \%$ \\
\hline$\gamma$-Decalactone & 30.87 & $0.02 \%$ & $1.95 \%$ & $1.13 \%$ & $0.63 \%$ & $1.71 \%$ \\
\hline Maltol & 25.01 & $0.04 \%$ & $7.60 \%$ & $1.78 \%$ & $5.18 \%$ & $1.66 \%$ \\
\hline Methyl cyclopentenolone & 23.12 & $0.02 \%$ & $0.91 \%$ & $1.59 \%$ & $1.38 \%$ & $1.33 \%$ \\
\hline Vanillin & 30.61 & $0.02 \%$ & $1.72 \%$ & $1.81 \%$ & $2.32 \%$ & $0.52 \%$ \\
\hline \multirow[t]{2}{*}{ Furaneol } & 23.16 & $0.03 \%$ & $2.63 \%$ & $1.66 \%$ & $1.81 \%$ & $1.00 \%$ \\
\hline & $\begin{array}{c}\text { Mean RT } \\
(\mathrm{n}=30)\end{array}$ & $\begin{array}{c}\text { RSD } \\
(\mathrm{n}=30)\end{array}$ & $\begin{array}{c}\text { RSD } \\
(\mathrm{n}=30)\end{array}$ & & & \\
\hline Benzene-d6 & 13.32 & $0.04 \%$ & $2.53 \%$ & & & \\
\hline n-Heptadecane & 31.89 & $0.02 \%$ & $2.97 \%$ & & & \\
\hline
\end{tabular}

Repeatability was determined for the retention times and peak areas. For analysis, 4 solutions and 1 blank sample were injected 6 times. RSD, relative standard deviation; PG, propylene glycol; VG, vegetable glycerin. 
Appendix Table A5.4: Recovery of the 10 flavorings selected for quantification, including the relative standard deviation (RSD, see Table A5.3).

\begin{tabular}{lcccccccc}
\hline \multicolumn{1}{c}{ PG/VG } & \multicolumn{2}{c}{$\mathbf{3 0 / 7 0}$} & \multicolumn{2}{c}{$\mathbf{3 0 / 7 0}$} & \multicolumn{2}{c}{$\mathbf{7 0 / 3 0}$} & \multicolumn{2}{c}{$\mathbf{7 0 / 3 0}$} \\
\multicolumn{1}{c}{ Conc. $(\boldsymbol{\mu g} / \mathbf{m L})$} & \multicolumn{2}{c}{$\mathbf{2 0}$} & \multicolumn{2}{c}{$\mathbf{8 0}$} & \multicolumn{2}{c}{$\mathbf{2 0}$} & \multicolumn{2}{c}{$\mathbf{8 0}$} \\
\hline Flavoring & Recovery & RSD & Recovery & RSD & Recovery & RSD & Recovery & RSD \\
\hline Cis-3-hexenol & $99.2 \%$ & $1.61 \%$ & $94.8 \%$ & $1.55 \%$ & $100.3 \%$ & $0.77 \%$ & $96.4 \%$ & $2.32 \%$ \\
Ethyl acetate & $104.5 \%$ & $1.34 \%$ & $101.3 \%$ & $1.32 \%$ & $103.9 \%$ & $0.82 \%$ & $101.7 \%$ & $2.10 \%$ \\
Ethyl butyrate & $98.7 \%$ & $1.98 \%$ & $96.2 \%$ & $1.49 \%$ & $100.6 \%$ & $1.77 \%$ & $98.6 \%$ & $2.17 \%$ \\
Ethyl maltol & $113.2 \%$ & $27.10 \%$ & $102.8 \%$ & $4.07 \%$ & $120.1 \%$ & $25.53 \%$ & $104.0 \%$ & $4.50 \%$ \\
Ethyl vanillin & $99.9 \%$ & $1.13 \%$ & $96.9 \%$ & $1.11 \%$ & $100.8 \%$ & $1.44 \%$ & $96.2 \%$ & $1.60 \%$ \\
$\gamma$-Decalactone & $92.4 \%$ & $1.95 \%$ & $93.6 \%$ & $1.13 \%$ & $91.6 \%$ & $0.63 \%$ & $94.4 \%$ & $1.71 \%$ \\
Maltol & $206.7 \%$ & $7.60 \%$ & $122.9 \%$ & $1.78 \%$ & $205.3 \%$ & $5.18 \%$ & $124.1 \%$ & $1.66 \%$ \\
Methyl cyclopentenolone & $103.1 \%$ & $0.91 \%$ & $95.0 \%$ & $1.59 \%$ & $100.5 \%$ & $1.38 \%$ & $96.1 \%$ & $1.33 \%$ \\
Vanillin & $102.0 \%$ & $1.72 \%$ & $98.1 \%$ & $1.81 \%$ & $102.2 \%$ & $2.32 \%$ & $98.1 \%$ & $0.52 \%$ \\
Furaneol & $115.7 \%$ & $2.63 \%$ & $114.3 \%$ & $1.66 \%$ & $108.1 \%$ & $1.81 \%$ & $107.1 \%$ & $1.00 \%$ \\
\hline
\end{tabular}

For each component, 6 injections were analyzed and results were averaged.

RSD, relative standard deviation; PG, propylene glycol; VG, vegetable glycerin.

Appendix Table A5.5: Sensitivity of the 10 flavorings selected for quantification.

\begin{tabular}{llllll}
\hline Flavoring & $\begin{array}{l}\text { Concentration } \\
(\boldsymbol{\mu g} / \mathbf{m l})\end{array}$ & $\begin{array}{l}\text { LOD } \\
\text { standard } \\
(\boldsymbol{\mu g} / \mathbf{m L})\end{array}$ & $\begin{array}{l}\text { LOQ } \\
\text { standard } \\
(\boldsymbol{\mu g} / \mathbf{m L})\end{array}$ & $\begin{array}{l}\text { LOD } \\
\mathbf{s a m p l e *} \\
(\mathbf{m g} / \mathbf{m L})\end{array}$ & $\begin{array}{l}\text { LOQ } \\
\mathbf{s a m p l e} \\
(\mathbf{m g} / \mathbf{m L})\end{array}$ \\
\hline Ethyl acetate & 10 & 0.09 & 0.29 & 0.01 & 0.03 \\
Ethyl butyrate & 10 & 0.19 & 0.57 & 0.02 & 0.06 \\
Cis-3-hexenol & 10 & 0.22 & 0.68 & 0.02 & 0.07 \\
Methyl cyclopentenolone & 10 & 0.19 & 0.58 & 0.02 & 0.06 \\
Vanillin & 10 & 0.13 & 0.41 & 0.01 & 0.04 \\
$\gamma$-Decalactone & 10 & 0.32 & 0.96 & 0.03 & 0.10 \\
Ethyl vanillin & 10 & 0.10 & 0.31 & 0.01 & 0.03 \\
Furaneol & 10 & 0.73 & 2.21 & 0.07 & 0.22 \\
Maltol & 30 & 0.82 & 2.47 & 0.08 & 0.25 \\
Ethyl maltol & 30 & 0.22 & 0.66 & 0.02 & 0.07 \\
\hline
\end{tabular}

$\mathrm{LOD}=3.3 *$ standard deviation / slope. $\mathrm{LOQ}=10 *$ standard deviation / slope.

LOD, limit of detection; LOQ, limit of quantification.

* Sample values represent LOD and LOQ in the e-liquid samples (i.e., $100 *$ the

LOD and LOQ in the flavoring standards, given a sample dilution of 100 times). 


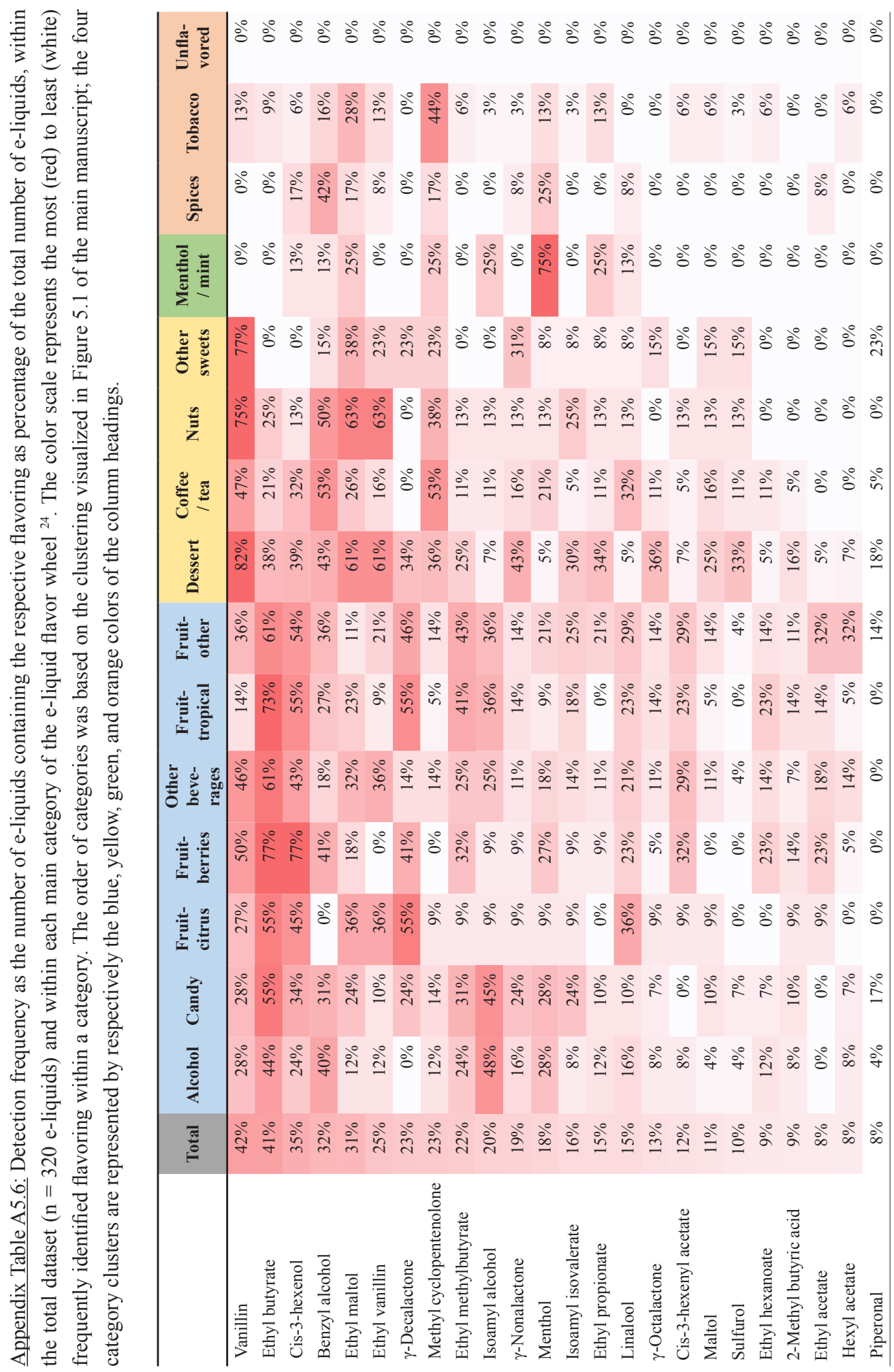




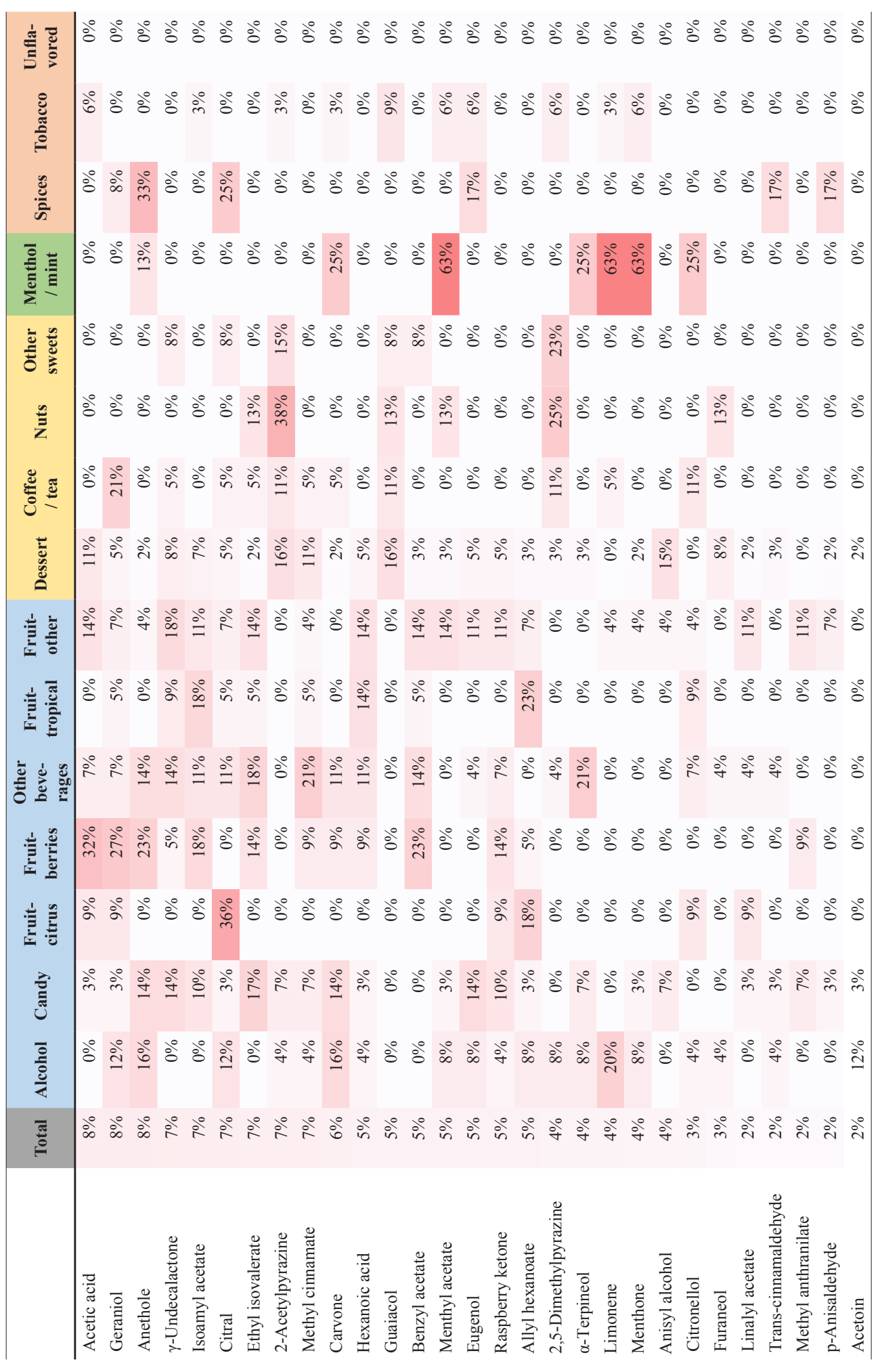




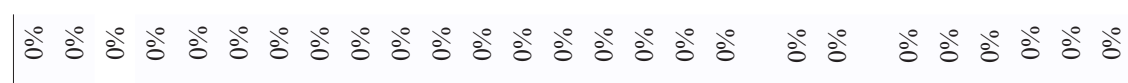

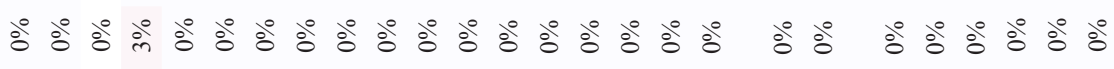

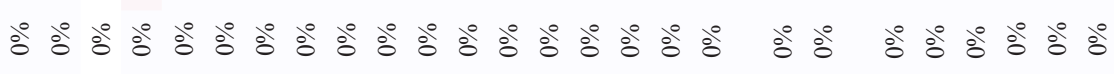

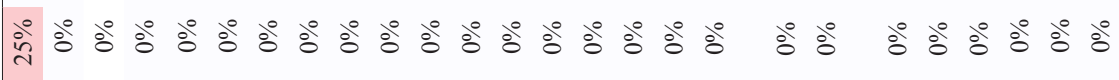

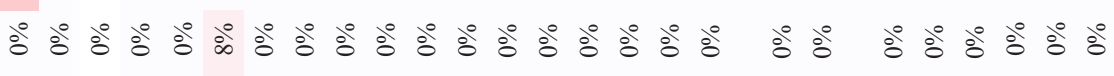

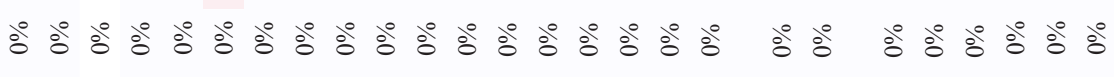

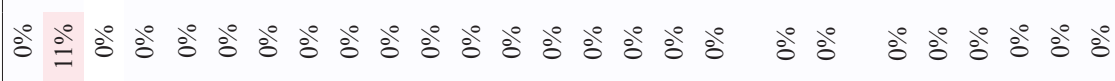

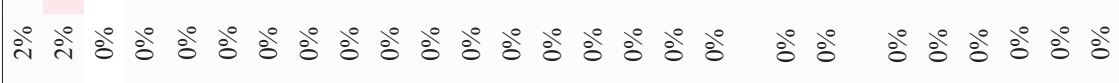

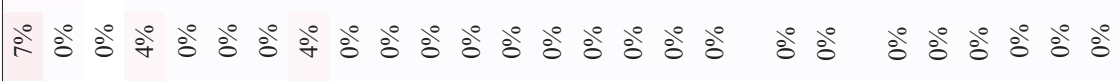

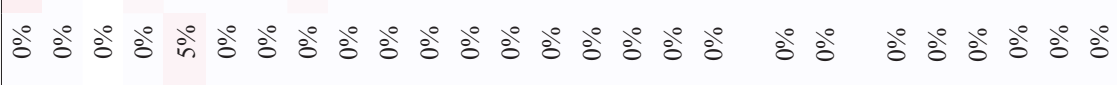

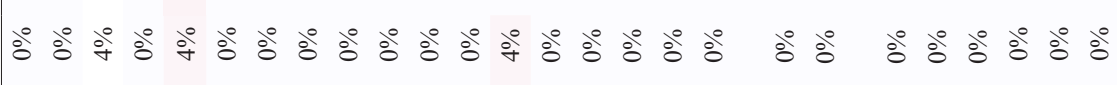

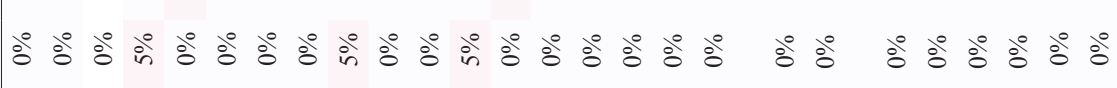

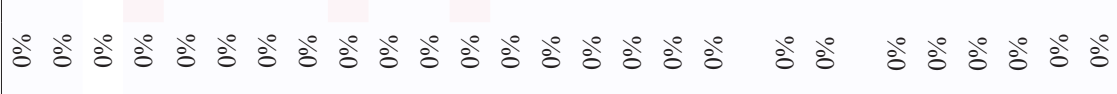

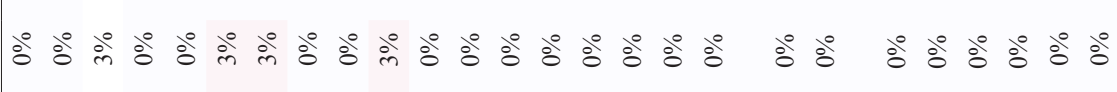

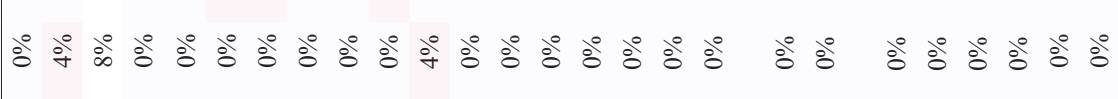

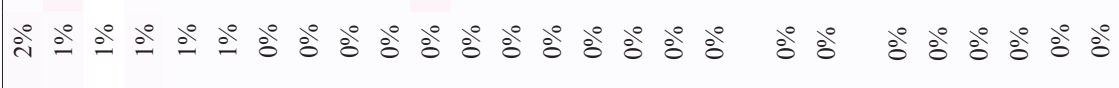

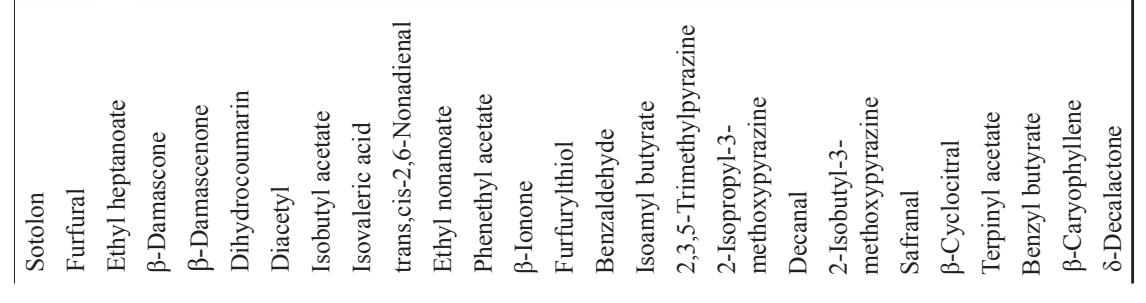




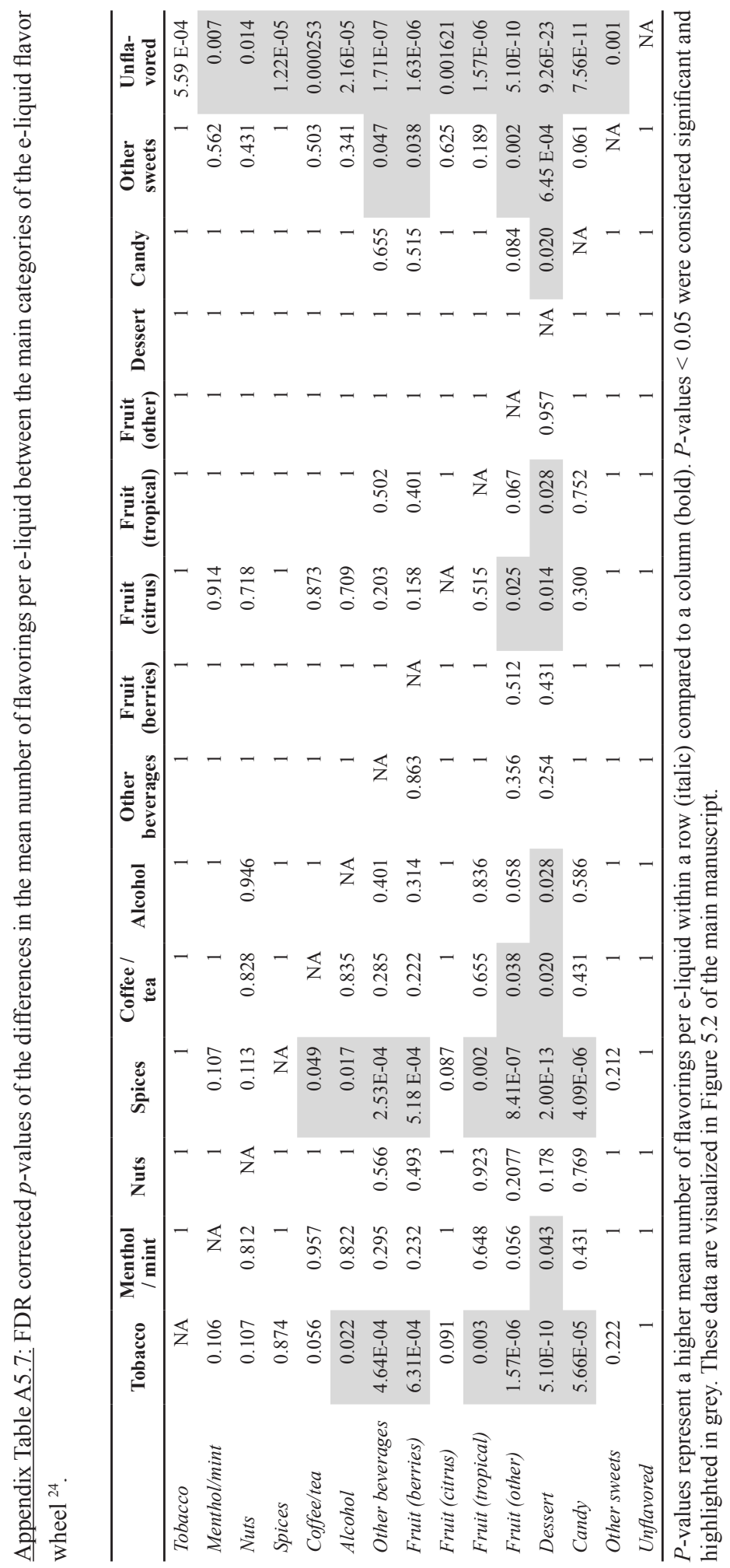


Appendix Table A5.8: Flavoring concentrations (median and range), if detected, for each individual flavor category and in the total data set $(\mathrm{n}=320$ e-liquids), including compound prevalence and concentrations.

\begin{tabular}{|c|c|c|c|c|c|c|c|}
\hline \multirow[t]{2}{*}{ Flavoring } & \multirow[t]{2}{*}{ Flavor category } & \multirow{2}{*}{$\begin{array}{l}\text { Prevalence } \\
>\text { LOD (n) }\end{array}$} & \multirow{2}{*}{$\begin{array}{l}\text { Prevalence } \\
>\text { LOQ (n) }\end{array}$} & \multicolumn{3}{|c|}{ Concentration $(\mathrm{mg} / \mathrm{mL})$} & \multirow[t]{2}{*}{ Mean RSD } \\
\hline & & & & Median & $\begin{array}{r}\text { Lower } \\
\text { limit }\end{array}$ & $\begin{array}{r}\text { Upper } \\
\text { limit }\end{array}$ & \\
\hline \multirow{13}{*}{ 咅 } & Tobacco & 4 & 4 & 1.6 & 0.5 & 3.3 & \\
\hline & Nuts & 6 & 6 & 1.9 & 0.5 & 11.6 & \\
\hline & Coffee/tea & 9 & 9 & 1.2 & 0.3 & 9.3 & \\
\hline & Alcohol & 7 & 6 & 0.8 & 0.0 & 7.5 & \\
\hline & Other beverages & 13 & 13 & 0.7 & 0.2 & 2.7 & \\
\hline & Fruit (berries) & 11 & 9 & 0.2 & 0.0 & 0.6 & \\
\hline & Fruit (citrus) & 3 & 3 & 0.8 & 0.8 & 5.3 & \\
\hline & Fruit (tropical) & 3 & 3 & 0.4 & 0.4 & 0.6 & \\
\hline & Fruit (other) & 10 & 10 & 0.8 & 0.3 & 1.6 & \\
\hline & Dessert & 50 & 50 & 2.9 & 0.4 & 13.5 & \\
\hline & Candy & 8 & 8 & 1.1 & 0.3 & 6.5 & \\
\hline & Other sweets & 10 & 10 & 1.6 & 0.2 & 9.8 & \\
\hline & TOTAL & 134 & 131 & 1.3 & 0.2 & 13.5 & $4.2 \%$ \\
\hline \multirow{13}{*}{ 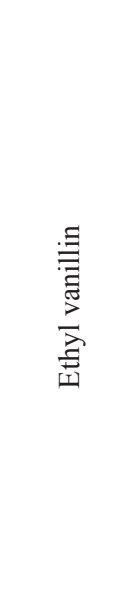 } & Tobacco & 4 & 4 & 1.4 & 1.0 & 1.9 & \\
\hline & Nuts & 5 & 5 & 0.5 & 0.3 & 5.6 & \\
\hline & Spices & 1 & 1 & 0.3 & 0.3 & 0.3 & \\
\hline & Coffee/tea & 3 & 3 & 4.2 & 2.4 & 4.8 & \\
\hline & Alcohol & 3 & 3 & 0.7 & 0.4 & 1.8 & \\
\hline & Other beverages & 10 & 10 & 1.1 & 0.2 & 4.5 & \\
\hline & Fruit (citrus) & 4 & 3 & 0.7 & 0.0 & 3.0 & \\
\hline & Fruit (tropical) & 2 & 2 & 0.2 & 0.2 & 0.3 & \\
\hline & Fruit (other) & 6 & 5 & 0.4 & 0.0 & 1.5 & \\
\hline & Dessert & 37 & 37 & 1.3 & 0.2 & 12.8 & \\
\hline & Candy & 3 & 3 & 0.4 & 0.2 & 8.9 & \\
\hline & Other sweets & 3 & 2 & 2.2 & 0.0 & 3.0 & \\
\hline & TOTAL & 81 & 78 & 1.1 & 0.2 & 12.8 & $4.1 \%$ \\
\hline
\end{tabular}




\begin{tabular}{|c|c|c|c|c|c|c|c|}
\hline \multirow{12}{*}{ 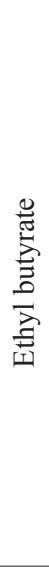 } & Tobacco & 3 & 3 & 0.3 & 0.1 & 0.3 & \\
\hline & Nuts & 2 & 2 & 0.3 & 0.1 & 0.6 & \\
\hline & Coffee/tea & 4 & 4 & 0.2 & 0.1 & 0.5 & \\
\hline & Alcohol & 11 & 11 & 0.2 & 0.0 & 2.2 & \\
\hline & Other beverages & 17 & 17 & 0.5 & 0.1 & 7.0 & \\
\hline & Fruit (berries) & 17 & 17 & 0.6 & 0.1 & 4.3 & \\
\hline & Fruit (citrus) & 6 & 6 & 0.5 & 0.1 & 0.9 & \\
\hline & Fruit (tropical) & 16 & 16 & 0.3 & 0.1 & 2.2 & \\
\hline & Fruit (other) & 17 & 17 & 0.5 & 0.1 & 2.8 & \\
\hline & Dessert & 23 & 23 & 0.3 & 0.1 & 1.3 & \\
\hline & Candy & 16 & 16 & 0.6 & 0.2 & 3.1 & \\
\hline & TOTAL & 132 & 132 & 0.4 & 0.1 & 7.0 & $7.3 \%$ \\
\hline \multirow{15}{*}{ 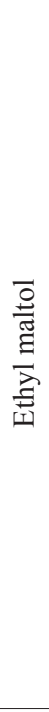 } & Tobacco & 9 & 9 & 2.8 & 0.8 & 13.6 & \\
\hline & Menthol/mint & 2 & 1 & 3.2 & 0.0 & 6.4 & \\
\hline & Nuts & 5 & 4 & 11.6 & 0.0 & 17.2 & \\
\hline & Spices & 2 & 2 & 5.7 & 1.1 & 6.6 & \\
\hline & Coffee/tea & 5 & 3 & 1.0 & 0.0 & 4.6 & \\
\hline & Alcohol & 3 & 3 & 8.4 & 8.3 & 18.3 & \\
\hline & Other beverages & 9 & 9 & 1.5 & 0.8 & 11.5 & \\
\hline & Fruit (berries) & 4 & 2 & 0.7 & 0.0 & 14.4 & \\
\hline & Fruit (citrus) & 4 & 2 & 0.3 & 0.0 & 0.6 & \\
\hline & Fruit (tropical) & 5 & 2 & 0.0 & 0.0 & 2.8 & \\
\hline & Fruit (other) & 3 & 2 & 1.5 & 0.0 & 2.7 & \\
\hline & Dessert & 37 & 28 & 3.1 & 0.0 & 17.3 & \\
\hline & Candy & 7 & 4 & 1.5 & 0.0 & 6.6 & \\
\hline & Other sweets & 5 & 5 & 1.3 & 0.6 & 14.2 & \\
\hline & TOTAL & 100 & 76 & 3.1 & 0.3 & 18.3 & $9.6 \%$ \\
\hline \multirow{9}{*}{ 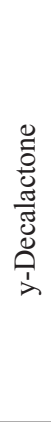 } & Other beverages & 4 & 4 & 1.0 & 0.0 & 1.4 & \\
\hline & Fruit (berries) & 9 & 9 & 0.2 & 0.1 & 0.8 & \\
\hline & Fruit (citrus) & 6 & 6 & 0.1 & 0.1 & 0.2 & \\
\hline & Fruit (tropical) & 12 & 12 & 0.2 & 0.1 & 2.0 & \\
\hline & Fruit (other) & 13 & 13 & 0.3 & 0.1 & 1.4 & \\
\hline & Dessert & 21 & 21 & 0.2 & 0.1 & 2.1 & \\
\hline & Candy & 7 & 6 & 0.1 & 0.0 & 0.2 & \\
\hline & Other sweets & 3 & 1 & 0.0 & 0.0 & 1.0 & \\
\hline & TOTAL & 75 & 72 & 0.2 & 0.0 & 1.4 & $7.7 \%$ \\
\hline
\end{tabular}




\begin{tabular}{|c|c|c|c|c|c|c|c|}
\hline \multirow{12}{*}{$\begin{array}{l}\frac{\overline{0}}{\frac{\pi}{\pi}} \\
\sum\end{array}$} & Tobacco & 2 & 2 & 0.8 & 0.5 & 1.0 & \\
\hline & Nuts & 1 & 1 & 0.4 & 0.4 & 0.4 & \\
\hline & Coffee/tea & 3 & 3 & 0.5 & 0.3 & 0.6 & \\
\hline & Alcohol & 1 & 1 & 0.8 & 0.8 & 0.8 & \\
\hline & Other beverages & 3 & 3 & 2.1 & 0.4 & 3.1 & \\
\hline & Fruit (citrus) & 1 & 1 & 0.4 & 0.4 & 0.4 & \\
\hline & Fruit (tropical) & 1 & 1 & 0.5 & 0.5 & 0.5 & \\
\hline & Fruit (other) & 4 & 4 & 1.0 & 0.2 & 1.2 & \\
\hline & Dessert & 15 & 15 & 0.5 & 0.2 & 1.6 & \\
\hline & Candy & 3 & 3 & 0.7 & 0.3 & 1.6 & \\
\hline & Other sweets & 2 & 2 & 0.4 & 0.4 & 0.4 & \\
\hline & TOTAL & 36 & 36 & 0.5 & 0.2 & 3.1 & $12.2 \%$ \\
\hline \multirow{14}{*}{ 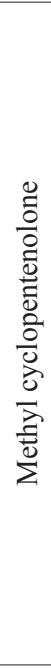 } & Tobacco & 14 & 14 & 1.4 & 0.4 & 6.2 & \\
\hline & Menthol/mint & 2 & 2 & 1.8 & 0.1 & 3.5 & \\
\hline & Nuts & 3 & 3 & 4.9 & 2.0 & 7.5 & \\
\hline & Spices & 2 & 2 & 1.4 & 0.4 & 2.5 & \\
\hline & Coffee/tea & 10 & 10 & 0.7 & 0.1 & 2.8 & \\
\hline & Alcohol & 3 & 2 & 0.9 & 0.0 & 2.1 & \\
\hline & Other beverages & 4 & 4 & 0.4 & 0.3 & 1.1 & \\
\hline & Fruit (citrus) & 1 & 1 & 0.1 & 0.1 & 0.1 & \\
\hline & Fruit (tropical) & 1 & 1 & 0.4 & 0.4 & 0.4 & \\
\hline & Fruit (other) & 4 & 3 & 0.4 & 0.0 & 1.1 & \\
\hline & Dessert & 22 & 22 & 1.0 & 0.1 & 7.4 & \\
\hline & Candy & 4 & 4 & 0.3 & 0.2 & 0.8 & \\
\hline & Other sweets & 3 & 3 & 0.5 & 0.4 & 0.6 & \\
\hline & TOTAL & 73 & 71 & 0.7 & 0.1 & 7.5 & $8.8 \%$ \\
\hline \multirow{5}{*}{ 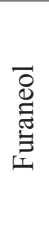 } & Nuts & 1 & 1 & 26.1 & 26.1 & 26.1 & \\
\hline & Alcohol & 1 & 1 & 19.6 & 19.6 & 19.6 & \\
\hline & Other beverages & 1 & 1 & 12.5 & 12.5 & 12.5 & \\
\hline & Dessert & 5 & 5 & 16.7 & 12.1 & 18.9 & \\
\hline & TOTAL & 8 & 8 & 17.6 & 12.1 & 26.1 & $6.1 \%$ \\
\hline
\end{tabular}




\begin{tabular}{|c|c|c|c|c|c|c|c|}
\hline \multirow{14}{*}{ 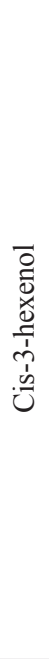 } & Tobacco & 2 & 2 & 0.8 & 0.7 & 0.8 & \\
\hline & Menthol/mint & 1 & 1 & 0.2 & 0.2 & 0.2 & \\
\hline & Nuts & 1 & 1 & 0.3 & 0.3 & 0.3 & \\
\hline & Spices & 2 & 2 & 0.2 & 0.2 & 0.2 & \\
\hline & Coffee/tea & 6 & 4 & 0.2 & 0.0 & 1.4 & \\
\hline & Alcohol & 6 & 6 & 0.6 & 0.3 & 2.0 & \\
\hline & Other beverages & 12 & 12 & 0.6 & 0.0 & 1.5 & \\
\hline & Fruit (berries) & 17 & 17 & 0.2 & 0.0 & 1.2 & \\
\hline & Fruit (citrus) & 5 & 5 & 0.3 & 0.1 & 0.6 & \\
\hline & Fruit (tropical) & 12 & 12 & 0.8 & 0.1 & 1.5 & \\
\hline & Fruit (other) & 15 & 13 & 0.3 & 0.0 & 2.9 & \\
\hline & Dessert & 24 & 22 & 0.1 & 0.0 & 1.5 & \\
\hline & Candy & 10 & 8 & 0.1 & 0.0 & 0.7 & \\
\hline & TOTAL & 113 & 105 & 0.3 & 0.0 & 2.9 & $6.1 \%$ \\
\hline \multirow{8}{*}{ 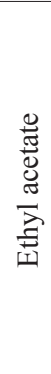 } & Spices & 1 & 1 & 0.4 & 0.4 & 0.4 & \\
\hline & Other beverages & 5 & 5 & 0.6 & 0.2 & 3.3 & \\
\hline & Fruit (berries) & 5 & 5 & 0.5 & 0.2 & 1.8 & \\
\hline & Fruit (citrus) & 1 & 1 & 0.6 & 0.6 & 0.6 & \\
\hline & Fruit (tropical) & 3 & 3 & 0.3 & 0.2 & 0.9 & \\
\hline & Fruit (other) & 9 & 9 & 0.3 & 0.1 & 2.1 & \\
\hline & Dessert & 3 & 3 & 0.3 & 0.2 & 0.3 & \\
\hline & TOTAL & 27 & 27 & 0.4 & 0.1 & 3.3 & $5.4 \%$ \\
\hline
\end{tabular}

Flavoring prevalence was reported as the number of e-liquids within a category in which the flavoring was detected (third column), and the number of e-liquids in which the flavoring was detected at a concentration higher than LOQ (fourth column). The RSDs of the individual flavoring concentrations were averaged over the whole dataset (final column). Statistical differences could not be determined due to unequal detection frequencies of flavorings between the categories.

RSD, relative standard deviation; LOD, limit of detection; LOQ, limit of quantification. 


\section{References}

1. Filippidis FT, Laverty AA, Gerovasili V, Vardavas CI. Two-year trends and predictors of e-cigarette use in 27 European Union member states. Tob Control. 2017;26(1):98-104.

2. US Department of Health and Human Services. E-cigarette use among youth and young adults: a report of the surgeon general. Atlanta, GA: US Department of Health and Human Services, Centers for Disease Control and Prevention, National Center for Chronic Disease Prevention and Health Promotion, Office on Smoking and Health; 2016.

3. Romijnders K, van Osch L, de Vries H, Talhout R. Perceptions and Reasons Regarding E-Cigarette Use among Users and Non-Users: A Narrative Literature Review. Int J Environ Res Public Health. $2018 ; 15(6)$.

4. Havermans A, Krüsemann EJZ, Pennings J, de Graaf K, Boesveldt S, Talhout R. Nearly 20000 e-liquids and 250 unique flavour descriptions: an overview of the Dutch market based on information from manufacturers. Tob Control. 2019:tobaccocontrol-2019-055303.

5. Polosa R, O'Leary R, Tashkin D, Emma R, Caruso M. The effect of e-cigarette aerosol emissions on respiratory health: a narrative review. Expert Rev Respir Med. 2019;13(9):899-915.

6. Goldenson NI, Leventhal AM, Simpson KA, Barrington-Trimis JL. A Review of the Use and Appeal of Flavored Electronic Cigarettes. Curr Addict Rep. 2019;6(2):98-113.

7. Rankin GD, Wingfors $\mathrm{H}$, Uski $\mathrm{O}$, et al. The toxic potential of a fourth-generation E-cigarette on human lung cell lines and tissue explants. J Appl Toxicol. 2019;39(8):1143-1154.

8. Prochaska JJ. The public health consequences of e-cigarettes: a review by the National Academies of Sciences. A call for more research, a need for regulatory action. Addiction. 2019;114(4):587-589.

9. Scientific Committee on Health Environmental and Emerging Risks (SCHEER). Additives used in tobacco products - Opinion II. 2016; 107. Available at: https:/ec.europa.eu/health/sites/ health/files/ scientific_committees/scheer/docs/scheer_o_001.pdf.

10. Krüsemann EJZ, Havermans A, Pennings JLA, de Graaf K, Boesveldt S, Talhout R. Comprehensive overview of common e-liquid ingredients and how they can be used to predict an e-liquid's flavour category. Tob Control. 2020:tobaccocontrol-2019-055447.

11. Erythropel HC, Jabba SV, DeWinter TM, et al. Formation of flavorant-propylene Glycol Adducts With Novel Toxicological Properties in Chemically Unstable E-Cigarette Liquids. Nicotine Tob Res. 2019;21(9):1248-1258.

12. Ruggirello M, Giordano M, Bertolino M, Ferrocino I, Cocolin L, Dolci P. Study of Lactococcus lactis during advanced ripening stages of model cheeses characterized by GC-MS. Food Microbiol. 2018;74:132-142.

13. Wen Y, Lopez R, Ferreira V. An automated gas chromatographic-mass spectrometric method for the quantitative analysis of the odor-active molecules present in the vapors emanated from wine. $J$ Chromatogr A. 2018;1534:130-138.

14. Ben Brahim S, Amanpour A, Chtourou F, Kelebek H, Selli S, Bouaziz M. Gas ChromatographyMass Spectrometry-Olfactometry To Control the Aroma Fingerprint of Extra Virgin Olive Oil from Three Tunisian Cultivars at Three Harvest Times. J Agric Food Chem. 2018;66(11):2851-2861.

15. Krusemann EJ, Visser WF, Cremers JW, Pennings J, Talhout R. Identification of flavour additives in tobacco products to develop a flavour library. Tob Control. 2018;27(1):105-111.

16. Herrington JS, Myers C. Electronic cigarette solutions and resultant aerosol profiles. J Chromatogr A. 2015;1418:192-199. 
17. Rawlinson C, Martin S, Frosina J, Wright C. Chemical characterisation of aerosols emitted by electronic cigarettes using thermal desorption-gas chromatography-time of flight mass spectrometry. J Chromatogr A. 2017;1497:144-154.

18. Leigh NJ, Lawton RI, Hershberger PA, Goniewicz ML. Flavourings significantly affect inhalation toxicity of aerosol generated from electronic nicotine delivery systems (ENDS). Tob Control. 2016;25(Suppl 2):ii81-ii87.

19. Aszyk J, Wozniak MK, Kubica P, Kot-Wasik A, Namiesnik J, Wasik A. Comprehensive determination of flavouring additives and nicotine in e-cigarette refill solutions. Part II: Gas-chromatography-mass spectrometry analysis. J Chromatogr A. 2017;1517:156-164.

20. Hutzler C, Paschke M, Kruschinski S, Henkler F, Hahn J, Luch A. Chemical hazards present in liquids and vapors of electronic cigarettes. Arch Toxicol. 2014;88(7):1295-1308.

21. Tierney PA, Karpinski CD, Brown JE, Luo W, Pankow JF. Flavour chemicals in electronic cigarette fluids. Tob Control. 2016;25(e1):e10-15.

22. Aszyk J, Kubica P, Wozniak MK, Namiesnik J, Wasik A, Kot-Wasik A. Evaluation of flavour profiles in e-cigarette refill solutions using gas chromatography-tandem mass spectrometry. J Chromatogr A. 2018;1547:86-98.

23. Omaiye EE, McWhirter KJ, Luo W, Tierney PA, Pankow JF, Talbot P. High concentrations of flavor chemicals are present in electronic cigarette refill fluids. Sci Rep. 2019;9(1):2468.

24. Krüsemann EJZ, Boesveldt S, de Graaf K, Talhout R. An E-Liquid Flavor Wheel: A Shared Vocabulary Based on Systematically Reviewing E-Liquid Flavor Classifications in Literature. Nicotine Tob Res. 2019;21(10):1310-1319.

25. Leffingwell \& Associates. Flavor-Base 9 - Tobacco Version for Windows XP/Vista/7\&8. 2013.

26. Benjamini Y, Hochberg Y. Controlling the False Discovery Rate: A Practical and Powerful Approach to Multiple Testing. J Roy Stat Soc B Met. 1995;57(1):289-300.

27. Hoffman AC, Salgado RV, Dresler C, Faller RW, Bartlett C. Flavour preferences in youth versus adults: a review. Tob Control. 2016;25(Suppl 2):ii32-ii39.

28. Romijnders KA, Krüsemann EJ, Boesveldt S, de Graaf K, de Vries H, Talhout R. E-Liquid Flavor Preferences and Individual Factors Related to Vaping: A Survey among Dutch Never-Users, Smokers, Dual Users, and Exclusive Vapers. Int J Environ Res Public Health. 2019;16(23):4661.

29. Stein YS, Antal MJ, Jones M. A study of the gas-phase pyrolysis of glycerol. J Anal Appl Pyrol. 1983;4(4):283-296.

30. Le Berre E, Beno N, Ishii A, Chabanet C, Etievant P, Thomas-Danguin T. Just noticeable differences in component concentrations modify the odor quality of a blending mixture. Chem Senses. 2008;33(4):389-395. 



\section{CHAPTER 6}

\section{E-liquid flavor preferences and individual factors}

related to vaping: A survey among Dutch

\section{never-users, smokers, dual users, and exclusive vapers}

Kim A.G.J. Romijnders*

Erna J.Z. Krüsemann*

Sanne Boesveldt

Kees de Graaf

Hein de Vries

Reinskje Talhout

* shared first authorship

Published in International Journal of Environmental Research and Public Health. 2019;16(23). https://doi.org/10.3390/ijerph16234661. 


\begin{abstract}
Appealing product characteristics, such as flavors, may stimulate e-cigarette use. Whereas switching to e-cigarettes may reduce harm for smokers, concerns exist about e-cigarette use among never-smokers. The role of flavors in the decision to switch to or refrain from vaping is unclear. This study used a bottom-up approach to investigate the relation between flavor preferences and individual factors related to vaping in various user groups. A cross-sectional survey was conducted among never-users $(n=407)$, smokers $(n=138)$, dual users $(n=122)$, and exclusive vapers $(n=61)$ in the Netherlands. Demographics, attractiveness of product characteristics, flavor preferences, and individual factors related to vaping (knowledge, trust, perceived susceptibility, attitude, social influence, deliberation, and intention) were assessed. Availability of different flavors was the most attractive characteristic of e-cigarettes. Dual users and exclusive vapers had most often used tobacco and menthol/mint flavors when they first started vaping. Compared to dual users, exclusive vapers currently used more sweet and fruit flavors. Never-users who were interested in trying an e-liquid flavor had more knowledge about and a more positive attitude towards e-cigarettes. Smokers who were interested in trying a flavor had a more positive attitude towards e-cigarettes and experienced the social influence towards not using e-cigarettes as less strong, than those who did not want to try any flavor. Hence, individual factors related vaping differed depending on whether never-users and smokers wanted to try an e-liquid flavor. This means that flavors may moderate differences found in individual factors related to vaping, or vice versa.
\end{abstract}




\section{Introduction}

Although vaping prevalence in the Netherlands is currently rather low (3\%) ${ }^{1}$, worldwide use of electronic cigarettes (e-cigarettes) increased worldwide in recent years ${ }^{2,3}$. The majority of e-cigarette users are former or current smokers ${ }^{4-6}$ and literature has showed that e-cigarette use (i.e., vaping) may be associated with smoking cessation and reduction ${ }^{7,8}$. In the Netherlands, e-cigarettes are more often used by daily smokers (12\%) compared to non-daily smokers (4\%). Worldwide, vaping is also becoming increasingly popular among adolescent non- and never-smokers ${ }^{3,9,10}$. The regulation of e-cigarettes in order to optimize public health benefits is challenging and is currently an important topic of debate. Compared to cigarette smoking, vaping may reduce harm among smokers ${ }^{11-14}$, but literature showed that e-cigarettes contain toxic ingredients ${ }^{11}$. In addition, concerns have been raised that vaping may contribute to nicotine addiction and renormalization of cigarette smoking in adolescent never-smokers ${ }^{3,15-}$ ${ }^{18}$. Consequently, from a public health perspective, the initiation of vaping by current non- and never-smokers, and thereby exposure to potentially toxic ingredients, should be prevented ${ }^{18}$.

Research is needed to better understand differences between initiation of e-cigarette use by current smokers versus non-smokers in order to inform regulators about policy-making regarding e-cigarettes in order to develop targeted health communication for smokers, nonsmokers, and e-cigarette users. Previous studies found differences in individual factors related to e-cigarettes among never-users, smokers, dual users, and e-cigarette users ${ }^{19}$. Individual factors that were found to differ included, for example, knowledge, perceived susceptibility, severity, trust, attitudes, deliberation, social influence, and intention ${ }^{19-23}$. Furthermore, literature showed that the importance of product characteristics such as design, price, and flavors may differ between adult smokers and adolescent non-smokers ${ }^{24-27}$. However, the relation between e-cigarette product characteristics and individual-level factors has been neglected.

A recent study hypothesized that there is an important interplay between individual-level factors and characteristics of tobacco products ${ }^{28}$. Since product characteristics (e.g., flavors, design, and price) influence e-cigarette appeal ${ }^{24-27}$ and may influence a person's attitude towards e-cigarettes ${ }^{24,28}$, such an interplay may also exist for e-cigarettes. However, thus far, most studies on e-cigarettes focused either on product characteristics ${ }^{29}$, or on socio-cognitive factors related to vaping behavior ${ }^{30}$. In contrast, researchers in the food and nutrition domain have already recognized the importance of the interaction between product characteristics and individuallevel decision-making factors in food choice ${ }^{31-33}$. For example, a model by Shepherd ${ }^{31}$ shows that food choice is influenced by the interaction between physical or chemical properties of food, such as flavors (product factor), and the individual's perception of and attitude towards those sensory properties (individual-level factors). Furthermore, flavors and other sensory properties are recognized as by far the most important factors in the acceptance and rejection of food products ${ }^{32}$.

Similarly, since e-liquid flavors are recognized as an important reason for e-cigarette use ${ }^{24}$, flavors may interact with individual-level factors related to vaping. Moreover, the availability of many different, mostly sweet, e-liquid flavors is an important reason for vaping among different types of users ${ }^{24,29}$. Research showed that for most e-cigarette users, and in particular for youth, the first and current e-liquid had a flavor other than tobacco ${ }^{26,34,35}$. In addition, flavors 
increase the probability of choosing e-cigarettes in an online discrete choice experiment among youth, for both never-users and ever-users of e-cigarettes ${ }^{36}$. Therefore, besides investigating product characteristics as reasons for e-cigarette use, additional research is needed to investigate the interaction between flavors as an e-cigarette product characteristic and individual factors related to vaping.

To increase our understanding of differences in e-cigarette appeal between user groups, this study firstly investigates which product characteristics are found attractive by Dutch neverusers, smokers, dual users, and exclusive vapers. Secondly, we aim to determine flavor preferences of Dutch never-users, smokers, dual users, and exclusive vapers. Thirdly, we aim to explore whether eight individual factors related to vaping differ between never-users and smokers who reported to be interested in trying an e-liquid flavor compared to those who reported not to be interested in trying any e-liquid flavor. 


\section{Materials and methods}

A cross-sectional survey was conducted in the Netherlands among never-users of e-cigarettes and cigarettes, cigarette smokers, dual users of e-cigarettes and cigarettes, and e-cigarette users. The survey was administered in May 2017 through the online survey panel Flycatcher, which is an ISO-certified independent research panel specialized in online research ${ }^{37}$. The study was approved by the Medical Ethics Committee of Zuyderland - Zuyd (17-N-88). The recruitment, participant characteristics, and survey were previously described by Romijnders et al. ${ }^{38}$.

\section{Recruitment and participant characteristics}

In total, 12750 invitations were sent to panelists who met the following inclusion criteria: being able to understand Dutch; being aware of e-cigarettes; aged 13-17 years (adolescents) or 18 years and older (adults). Sample size was determined based on a power of $80 \%$ to identify a minimal difference of 1 on a 7-point Likert scale for attitude (based on previous literature ${ }^{39}$ ) as significant at $p<0.05$. The sample cannot be considered representative of the Dutch population, as oversampling for the smokers and e-cigarette users was performed in order to have sufficient observations. Participants were asked to provide consent before the start of the survey. Parents of panelists under the age of 18 had previously provided consent for participation of their child in research questionnaires. Overall, 1307 surveys were completed and the response rate was 10.3\% ( $69.7 \%$ for adults, $\mathrm{n}=694 ; 5.2 \%$ for adolescents, $\mathrm{n}=613$ ). For the current study, respondents were eligible if they met the definition of one of the following user groups (see Appendix Table A6.1 for the survey items used): never-users are participants who reported to never have smoked and never used an e-cigarette; smokers are participants who reported to currently exclusively use cigarettes on a daily or weekly basis; dual users are participants who reported to currently simultaneously use cigarettes on a daily or weekly basis and e-cigarettes on a daily or weekly basis; vapers are participants who reported to currently exclusively use e-cigarettes on a daily or weekly basis ${ }^{40}$.

It should be noted that these definitions, similar to studies performed before ${ }^{39}$, include individuals who had no history with smoking prior to becoming an exclusive vaper. In addition, and due to the cross-sectional nature of the data, no transitory phases for dual users and exclusive vapers can be determined ${ }^{40}$. We aimed for mutually exclusive groups. Hence, as the group of exclusive vapers may also include former smokers and the group of exclusive smokers may also include former vapers, former users were not included as a separate group. An overview of the items used to determine whether respondents met our definitions can be found in Appendix Table A6.1. In total, 728 participants met the eligibility criteria of this study. Of those, 394 were adults (62.4\% female) and 334 were adolescents (46.7\% female).

\section{Survey}

The current study included measures on basic demographics, attractiveness of e-cigarettes, flavor preferences, and individual factors related to vaping. The survey included routing to ensure that participants were asked about relevant items only (e.g., never-users were not asked which flavor their first e-cigarette had). A full overview of the items and concepts is available in the Appendix. First, participants were asked about basic demographics, and smoking and vaping characteristics 
19,24,40-44. Educational level was determined based on the Dutch version of International Standard Classification of Education (ISCED) ${ }^{45}$.

Second, participants were asked to evaluate the attractiveness of eight product characteristics of e-cigarettes from a predetermined list using a check all that apply (CATA) approach that was based on previous research ${ }^{19,24}$. For the items that were used to assess demographics and attractiveness of product characteristics, see Appendix Table A6.1.

Third, interest in trying an e-liquid flavor (for never-users and smokers), and the first and current e-liquid flavors used (for dual and e-cigarette users) were assessed. For the items that were used to assess flavor preferences, see Appendix Table A6.2. To assess flavor interest among never-users and smokers, multiple flavor categories ${ }^{46}$ (CATA) or the option "none of the flavors" were selected. If the latter answer option was selected, no flavor category could be selected simultaneously. E-cigarette users and dual users were asked about their current and first e-liquid flavor used: "Which flavor do you currently use most? If possible, please specify the name of the flavor." and "Which flavor did you try first? If possible, please specify the name of the flavor." For both current and first flavor used, dual users and e-cigarette users could select only one flavor category ${ }^{46}$ and had to specify their choice through an open question. The answer options for never-users, smokers, dual users and e-cigarette users were: tobacco, menthol/ mint, nuts, herbs, spices, coffee/tea, cocktails, alcohol, other, sodas, sweet (chocolate, vanilla, desserts, or other), fruit, milk products, candy, floral, unflavored, and none of the flavors. The closed answers options that were used to assess flavor preference in all user groups were recoded according to the 13 main categories of the recently published e-liquid flavor wheel ${ }^{47}$, except for the option "none of the flavors". Recoding the reported flavor preferences resulted in the reported flavor preferences resulted in the following 13 main categories: tobacco (survey item: tobacco), menthol/mint (survey item: menthol/mint), nuts (survey item: nuts), spices (survey items: herbs, spices), coffee/tea (survey items: coffee; tea), alcohol (survey items: alcohol, cocktail; alcohol, other), other beverages (survey items: soda; sweet, other), fruit (survey item: fruit), dessert (survey items: sweet, dessert; milk product), other sweets (survey items: sweet, chocolate; sweet, vanilla), candy (survey items: sweet, candy), other flavors (survey items: floral; other) and unflavored (survey item: unflavored). Open answers from dual and e-cigarette users were assessed by two authors (KR and $\mathrm{EK}$ ) to support recoding of the closed answers according to the categories of the e-liquid flavor wheel ${ }^{47}$. In some cases, multiple survey items were associated with one flavor category (e.g., the survey answer options "sweet, chocolate" and "sweet, vanilla" were both recoded to the other sweets flavor category). For equal weight of the flavor categories, each participant could obtain a maximum score of " 1 " for each flavor category. Thus, participants who reported being interested in both chocolate and vanilla-flavored e-liquids received a total score of " 1 " for the other sweets flavor category.

Fourth, individual factors related to vaping were assessed (see Appendix Table A6.2 for the items that were used). Evidence-based knowledge about smoking and vaping was measured with 12 statements that were either correct or incorrect. We considered evidence-based knowledge as knowledge that is based on scientific consensus - that is, information provided by the Dutch National Institute of Public Health and the Environment (RIVM) and previous research 19,48,49. The knowledge items were summed ( 1 = correct, 0 = incorrect; "I don't know" was categorized as incorrect). Furthermore, a 7-point Likert scale was used to assess trust in information (two 
items) ${ }^{50}$, perceived susceptibility towards e-cigarettes (three items: item A, item B, item C) ${ }^{51,52}$, severity related to vaping (four items ${ }^{51,52}$ ), attitude towards e-cigarettes (four items) ${ }^{39,48}$, social influence (one item) ${ }^{53}$, deliberation about vaping (three items) ${ }^{48}$, and intention to start using e-cigarettes (one item). A scale was computed for trust in information, severity related to vaping, attitude towards e-cigarettes, and deliberation of the pros and cons of vaping, by averaging the scores of the two items for trust (Cronbach's $\alpha=0.915$ ), the four items for severity (Cronbach's $\alpha=0.639$ ), the four items of attitude (Cronbach's $\alpha=0.927$ ), and the three items for deliberation (Cronbach's $\alpha=0.656$ ). No scale could be computed for perceived susceptibility towards e-cigarettes (Cronbach's $\alpha \leq 0.6$ ) - thus, for each user group, the three mean scores for perceived susceptibility towards e-cigarettes and the three mean scores for perceived susceptibility towards cigarettes (for each individual survey item) were used. Similarly, for each user group, the mean score for each item regarding intention and social influence was determined.

\section{Data analysis}

IBM statistics SPSS V.24 (IBM, Armonk, NY, USA) ${ }^{54}$ was used for data analysis. No data were excluded. Attractiveness of product characteristics, and the e-liquid flavor categories preferred to try (for never-users and smokers, excluding those who selected the answer option "I don't want to try any flavor") and firstly and currently used (for dual and exclusive vapers) were analyzed using frequencies. Flavor preferences were presented in a pie chart as percentage of the total number of responses.

Spearman correlation analyses showed that age, gender, and level of education were significantly associated with individual factors related to vaping $(p<0.05)$. However, these Spearman correlations were small, ranging from -0.211 to 0.169 . Age, level of education, and gender were therefore excluded from further analyses due to small or non-significant correlations.

Individual factors were compared between both never-users and smokers interested in trying an e-liquid flavor and those not interested in trying a flavor (answer option: "none of the flavors") using $t$-tests. Results were considered significant if $p<0.05$. 


\section{Results}

Of the 728 never-users, smokers, dual users, and exclusive e-cigarette users, $23.7 \%$ was highly educated $(50.0 \%$ low education level and $26.2 \%$ middle education level), and the average age was $34.1 \pm 20.2(\min =13, \max =84)($ see $\underline{\text { Table } 6.1})$.

\section{Attractiveness of product characteristics}

Table 6.1 shows attractiveness of e-cigarette product characteristics, stratified by user group. From the e-cigarette product characteristics assessed, all groups reported flavors as the most attractive.

\section{E-liquid flavor preferences}

Of the 407 never-users, $68 \%$ selected the option "none of the flavors" ( $n=278$ participants) and $32 \%$ selected to be interested in trying one or more e-liquid flavors ( $\mathrm{n}=248$ responses $)$. Of the 138 smokers, $20 \%(n=27$ participants $)$ was interested in none of the flavors and $80 \%(n=208$ responses) selected to be interested in trying one or more e-liquid flavor categories.

Figure 6.1 shows e-liquid flavor preferences as percentage of each flavor category for never-users and smokers. Never-users were mostly interested in trying e-liquid flavors from the menthol/mint (19\% of the 248 responses) and sweet categories, such as other sweets (19\%) and fruit (14\%). Smokers were mostly interested in e-liquids with tobacco flavor (30\%), followed by menthol/mint (18\%) and other sweets $(9 \%)$.

Of the 122 dual users, 120 reported the flavor of their first e-cigarette used and 121 reported the flavor they currently use (see Figure 6.2). Of the 61 exclusive vapers, 58 reported the flavor of both their first and current e-cigarette. Among dual users, the most frequently reported flavors of their current and first e-cigarette used were similar: tobacco (52\% vs. 53\%), menthol/ mint $(26 \%$ vs. $27 \%)$, other sweets $(10 \%$ vs. $11 \%)$, and fruit (7\% vs. $6 \%)$. Among exclusive vapers, differences were observed in the most frequently reported flavors of their current and first e-cigarette used: tobacco (43\% vs. 53\%), menthol/mint (19\% vs. $28 \%)$, and fruit (14\% vs. 9\%) and other sweets (14\% vs. $7 \%)$. 
Table 6.1: Participants' demographics and the attractiveness of e-cigarette product characteristics. Data are presented for adult and adolescent never-users $(n=407)$, smokers $(n=138)$, dual users $(n=122)$, and exclusive vapers $(n=61)$.

\begin{tabular}{llllll}
\hline $\begin{array}{l}\text { Participants' demographics and the } \\
\text { attractiveness of e-cigarette product } \\
\text { characteristics }\end{array}$ & $\begin{array}{l}\text { Never- } \\
\text { users } \\
(\mathbf{n}=\mathbf{4 0 7})\end{array}$ & $\begin{array}{l}\text { Smokers } \\
(\mathbf{n = 1 3 8})\end{array}$ & $\begin{array}{l}\text { Dual users } \\
(\mathbf{n = 1 2 2})\end{array}$ & $\begin{array}{l}\text { Exclusive } \\
\text { vapers } \\
(\mathbf{n}=\mathbf{6 1})\end{array}$ \\
\hline Mean age (SD) & $31(18.6)^{\mathrm{D}}$ & $35(20.6)$ & $37(18.8)^{\mathrm{N}}$ & $37(19.4)$ \\
\hline Gender & Male & $44.0 \%$ & $37.7 \%$ & $53.3 \%$ & $49.2 \%$ \\
& Female & $56.0 \%$ & $62.3 \%$ & $46.7 \%$ & $50.8 \%$ \\
\hline Education & Low & $52.1 \%$ & $52.9 \%$ & $43.4 \%$ & $39.3 \%$ \\
& Middle & $20.6 \% \mathrm{D}^{\mathrm{D}, \mathrm{E}}$ & $30.4 \%$ & $35.2 \% \mathrm{~N}^{\mathrm{N}}$ & $44.3 \% \mathrm{~N}$ \\
& High & $27.3 \%$ & $16.7 \%$ & $21.3 \%$ & $16.4 \%$ \\
\hline $\begin{array}{l}\text { Attractive } \\
\text { characteristics } \\
\text { of e-cigarettes } \\
(\%)\end{array}$ & All the different flavors & $10.3 \%$ & $30.4 \%$ & $34.4 \%$ & $68.9 \%$ \\
& The product looks nice & $6.6 \%$ & $19.6 \%$ & $22.1 \%$ & $44.3 \%$ \\
& The nicotine level can be varied & $4.7 \%$ & $13.8 \%$ & $15.6 \%$ & $31.1 \%$ \\
& It is possible to alter the setting & $3.7 \%$ & $10.9 \%$ & $12.3 \%$ & $24.6 \%$ \\
& $\begin{array}{l}\text { of the e-cigarette to my wishes } \\
\text { Its varying designs }\end{array}$ & $3.2 \%$ & $9.4 \%$ & $10.7 \%$ & $21.3 \%$ \\
& $\begin{array}{l}\text { You can blow nice smoke } \\
\text { clouds with it (cloud chasing) }\end{array}$ & $2.5 \%$ & $7.2 \%$ & $8.2 \%$ & $16.4 \%$ \\
& Price of the product & $2.0 \%$ & $5.8 \%$ & $6.6 \%$ & $13.1 \%$ \\
& Price of the e-liquids & $2.0 \%$ & $5.8 \%$ & $6.6 \%$ & $13.1 \%$ \\
\hline
\end{tabular}

$\mathrm{N}, \mathrm{D}, \mathrm{E}$ Superscripts indicate significant differences in a row between user groups $(p<0.05)$, with $\mathrm{N}=$ never-users, $\mathrm{D}=$ dual users, and $\mathrm{E}=$ exclusive vapers. Significant differences between user groups were determined for age, gender, and education using Bonferroni post-hoc corrections. General note: due to rounding, percentages may not add up to $100 \%$. 


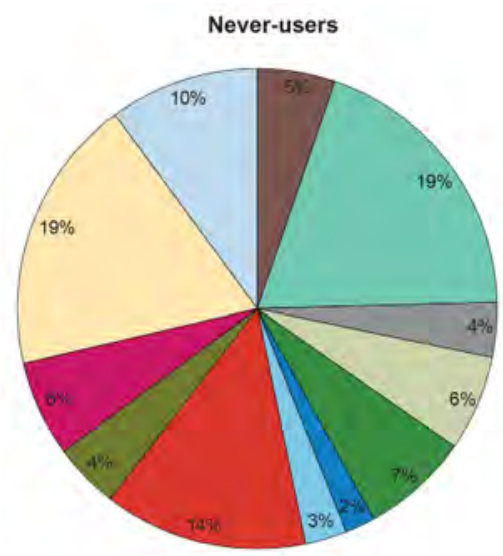

Total $=248$

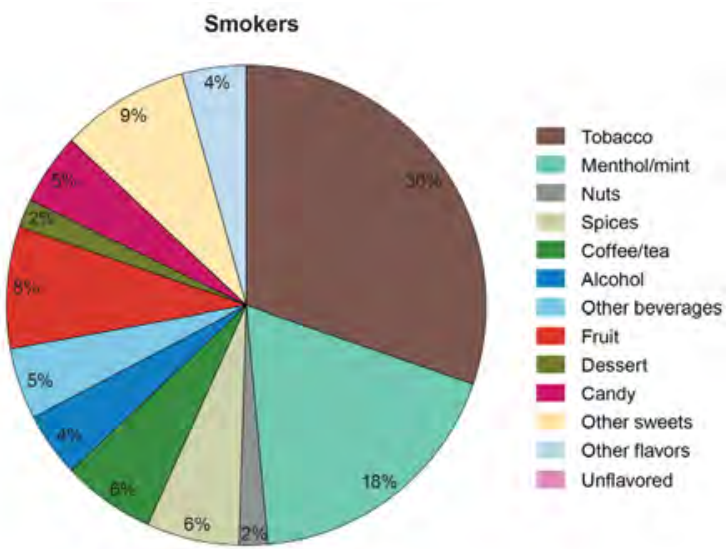

Total $=208$

Figure 6.1: Interest in trying e-liquid flavors among never-users (left) and smokers (right). Never-users $(\mathrm{n}=278 ; 68 \%)$ and smokers $(\mathrm{n}=27 ; 20 \%)$ who selected the option "none of the flavors" were excluded from this visualization; hence, the pie charts visualize 248 responses from $32 \%$ of the never-users and 208 responses from $80 \%$ of the smokers.. Data are presented as percentages of the total number of responses, not of the total sample sizes. 

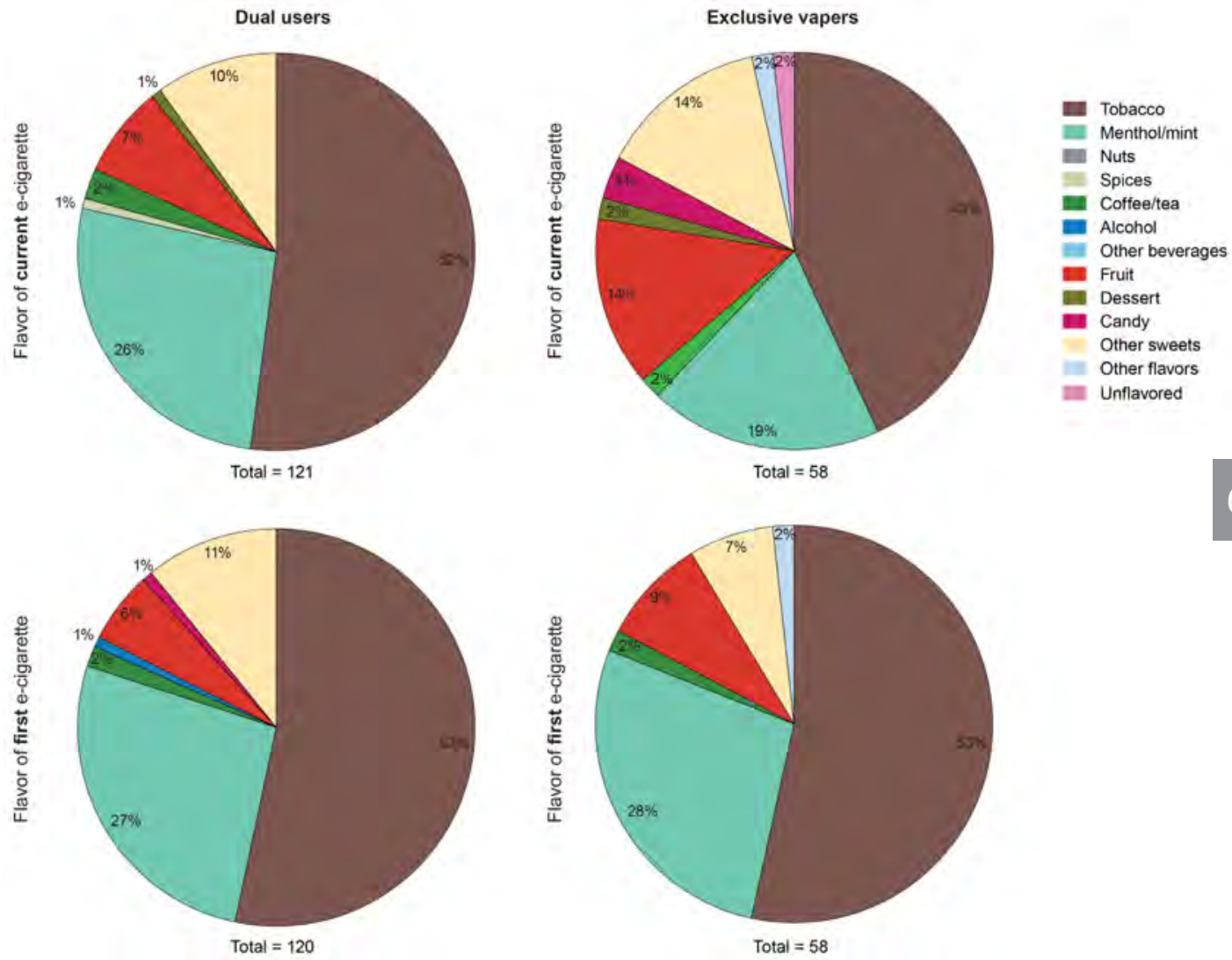

Figure 6.2: Flavors used at current (top) and first (bottom) e-cigarette exposure among dual users (left) and exclusive vapers (right). Participants could select only one flavor category to indicate the flavor in their current and first e-cigarette used. Data are presented as percentages of the total number of responses, not of the total sample sizes. 


\section{Individual factors related to vaping}

Table 6.2 shows differences in individual factors related vaping between never-users and smokers. In addition, differences within the groups of never-users and smokers between those who were interested in trying an e-liquid flavor and those who did not want to try any flavor are shown. Never-users who were interested in trying a flavor had significantly less knowledge about e-cigarettes compared to never-users who did not want to try any e-liquid flavor ( $p<$ $0.05)$. Not surprisingly, never-users and smokers who were interested in trying a flavor were significantly more positive towards e-cigarettes and had a significant higher intention to start vaping, compared to never users and smokers who reported not wanting to try any e-liquid flavor $(p<0.05)$, within both never-users and smokers. Never-users who were interested in trying a flavor reported a lower perceived susceptibility (item C) than never-users who did not want to try a flavor $(p<0.05)$. In addition, smokers who were interested in trying a flavor considered the social influence towards not using e-cigarettes as less strong, which means that the smokers who were not interested in trying an e-cigarette flavor more often find that society thinks that one should not vape $(p<0.05)$. 
Table 6.2: Individual factors related to vaping. Data are presented for never-users and smokers._

\begin{tabular}{|c|c|c|c|c|}
\hline \multicolumn{3}{|l|}{$\begin{array}{l}\text { Individual factors } \\
\text { related to vaping }\end{array}$} & \multirow{2}{*}{$\begin{array}{l}\begin{array}{l}\text { Never-users } \\
(\mathbf{n}=\mathbf{4 0 7})\end{array} \\
9.3(1.5) *\end{array}$} & \multirow{2}{*}{$\begin{array}{l}\begin{array}{c}\text { Smokers } \\
(\mathbf{n}=\mathbf{1 3 8})\end{array} \\
8.4(1.8) *\end{array}$} \\
\hline \multirow{3}{*}{$\begin{array}{l}\text { Knowledge about } \\
\text { e-cigarettes and } \\
\text { cigarettes (SD) }\end{array}$} & & Overall & & \\
\hline & & Those interested in trying a flavor & $8.9(1.7)^{\circ}$ & $8.4(1.7)$ \\
\hline & & Those who did not want to try any flavor & $9.4(1.4)^{\circ}$ & $8.3(2.1)$ \\
\hline \multirow{3}{*}{$\begin{array}{l}\text { Trust in } \\
\text { information (SD) }\end{array}$} & & Overall & $5.2(1.1)$ & $4.9(1.4)$ \\
\hline & & Those interested in trying a flavor & $5.2(1.0)$ & $4.9(1.4)$ \\
\hline & & Those who did not want to try any flavor & $5.2(1.4)$ & $5.1(1.5)$ \\
\hline \multirow{9}{*}{$\begin{array}{l}\text { Perceived } \\
\text { susceptibility about } \\
\text { vaping (SD) }\end{array}$} & A & Overall & $4.9(1.3)^{*}$ & $4.3(1.2) *$ \\
\hline & & Those interested in trying a flavor & $4.8(1.3)$ & $4.2(1.2)$ \\
\hline & & Those who did not want to try any flavor & $4.9(1.3)$ & $4.4(1.2)$ \\
\hline & $\mathrm{B}$ & Overall & $5.0(1.2)^{*}$ & $4.3(1.2) *$ \\
\hline & & Those interested in trying a flavor & $4.8(1.2)$ & $4.3(1.2)$ \\
\hline & & Those who did not want to try any flavor & $5.1(1.2)$ & $4.5(1.2)$ \\
\hline & $\mathrm{C}$ & Overall & $4.9(1.2)^{*}$ & $4.3(1.2) *$ \\
\hline & & Those interested in trying a flavor & $4.8(1.3)^{\circ}$ & $4.2(1.2)$ \\
\hline & & Those who did not want to try any flavor & $5.0(1.2)^{\circ}$ & $4.6(1.2)$ \\
\hline \multirow{3}{*}{$\begin{array}{l}\text { Severity of vaping } \\
\text { (SD) }\end{array}$} & & Overall & $4.6(1.1)^{*}$ & $4.4(1.1) *$ \\
\hline & & Those interested in trying a flavor & $4.6(1.1)$ & $4.4(1.2)$ \\
\hline & & Those who did not want to try any flavor & $4.6(1.1)$ & $4.1(1.1)$ \\
\hline \multirow{3}{*}{$\begin{array}{l}\text { Attitude towards } \\
\text { e-cigarettes (SD) }\end{array}$} & & Overall & $2.1(1.1)^{*}$ & $3.5(1.1) *$ \\
\hline & & Those interested in trying a flavor & $2.6(1.2)^{\circ}$ & $3.7(1.0)^{\circ}$ \\
\hline & & Those who did not want to try any flavor & $1.9(1.0)^{\circ}$ & $2.9(1.2)^{\circ}$ \\
\hline \multirow{3}{*}{\multicolumn{2}{|c|}{$\begin{array}{l}\text { Social influence } \\
\text { (SD) }\end{array}$}} & Overall & $5.1(1.7) *$ & $4.4(1.5) *$ \\
\hline & & Those interested in trying a flavor & $4.9(1.7)$ & $4.2(1.5)^{\circ}$ \\
\hline & & Those who did not want to try any flavor & $5.2(1.7)$ & $5.1(1.2)^{\circ}$ \\
\hline \multirow{3}{*}{\multicolumn{2}{|c|}{$\begin{array}{l}\text { Deliberation on the } \\
\text { pros and cons of } \\
\text { e-cigarette use (SD) }\end{array}$}} & Overall & $2.8(1.5)$ & $3.0(1.5)$ \\
\hline & & Those interested in trying a flavor & $2.9(1.6)$ & $3.1(1.4)$ \\
\hline & & Those who did not want to try any flavor & $2.7(1.5)$ & $2.7(1.7)$ \\
\hline \multirow{3}{*}{\multicolumn{2}{|c|}{$\begin{array}{l}\text { Intention to start } \\
\text { vaping (SD) }\end{array}$}} & Overall & $1.2(0.8) *$ & $2.5(1.7) *$ \\
\hline & & Those interested in trying a flavor & $1.4(1.1)^{\circ}$ & $2.7(1.7)^{\circ}$ \\
\hline & & Those who did not want to try any flavor & $1.1(0.6)^{\circ}$ & $1.6(1.4)^{\circ}$ \\
\hline
\end{tabular}

* Indicates significant differences $(p<0.05)$ in a row between user groups. ${ }^{\circ}$ Indicates a within-group significant difference $(p<0.05)$ between those who were interested in trying any e-liquid flavor and those who were not interested in trying any e-liquid flavor. Knowledge was determined using 12 statements; a higher score represented more knowledge, with $0=$ no correct answers and $12=$ correct answers for all statements. Trust was assessed with two items, using a 7-point Likert scale; $1=$ low to $7=$ high level of trust in information provided. Perceived susceptibility assessed the chance of developing cancer as a result of vaping with three statements: (A) If I vape, then my risk of developing some form of cancer during my lifetime is...; (B) I think that if I vape, my risk of developing some form of cancer during my lifetime is ...; (C) My feeling is that if I vape, the risk of developing some form of cancer during my lifetime is... Scores represent $1=$ low to $7=$ high perception of cognitive risk of health risks related to e-cigarette use. Severity was assessed with four items using a 7-point Likert scale, with $1=$ very bad and $7=$ not bad at all. Attitude was assessed with four items using a 7 -point Likert scale, with $1=$ very negative towards e-cigarette use and $7=$ very positive 
towards e-cigarette use. Deliberation was assessed with three items using a 7-point Likert scale, with $1=$ no deliberation about e-cigarette use and $7=$ very extensive deliberation about e-cigarette use. Intention to start vaping was assessed using a 7-point Likert scale, with $1=$ very low intention to start vaping and $7=$ very high intention to start vaping. 


\section{Discussion}

This study shows that the availability of different flavors was reported to be the most attractive product characteristic of e-cigarettes by all user groups, and that flavor preferences differ between never-users, smokers, dual users, and exclusive vapers. The first e-cigarette used by dual users and vapers mostly had a tobacco or menthol/mint flavor, but compared to dual users, we observed that exclusive vapers currently use more sweet and fruit-flavored e-liquids than dual users. While tobacco was the most appealing flavor category among smokers, never-users were mostly interested in trying menthol/mint and sweet-flavored e-liquids. In addition, individual factors related to vaping differed within the groups of never-users and smokers. That is, neverusers interested in trying a flavor had less knowledge about cigarettes and e-cigarettes than those who did not want to try any flavor. Attitude was more positive, and intention to start vaping was higher among both never-users and smokers who were interested in trying a flavor compared to those not interested in trying a flavor. Perceived susceptibility of health consequences was lower among never-users who were interested in trying a flavor, and social influence regarding not using e-cigarettes was lower among smokers who were interested in trying a flavor. Thus, similarly to the role of flavors in food choice ${ }^{31,32}$, our results indicate that interest in flavors may moderate the differences in individual factors related to vaping.

While concerns have been raised about potential e-cigarette use among never-users $3,9,55$, the never-users in our study had a low intention to start vaping and more than two-third (68\%) of the never-users did not want to try any e-liquid flavor. However, nearly one-third of the neverusers were still interested in trying an e-liquid flavor. Not surprisingly, they perceived a lower susceptibility towards negative health consequences of vaping, had a more positive attitude towards e-cigarettes, less knowledge about cigarettes and e-cigarettes, and a higher intention to start vaping than never-users who did not want to try any flavor. It should, however, be noted that a causal relation between these findings was not examined. For example, never-users could report to find e-liquid flavors interesting because they were already interested in trying e-cigarettes. On the other hand, they may have become interested in trying e-cigarettes because of the appealing flavors that they recognize from palatable food products. This means that being interested in flavors has a positive effect on the decision to start using e-cigarettes, or vice versa. Nevertheless, our findings regarding the interest of never-users in e-liquid flavors indicate that never-users may be vulnerable to flavor marketing of e-cigarettes ${ }^{26,27,56,57}$. For example, marketing of appealing e-liquid flavors may make never-users even more positive towards vaping, thereby potentially influencing their choice to initiate or refrain from vaping ${ }^{19}$. This suggests that the reverse can also be true: adapting product characteristics, for example restricting e-liquid flavors or regulating other product characteristics, may reduce attractiveness and consequently make never-users more negative about vaping. Some characteristics of e-liquids are currently regulated in the Netherlands ${ }^{58,59}$, yet legislation regarding e-liquid flavors currently does not exist. Further research is needed to help regulators decide whether and how the regulation of e-liquid flavors can improve public health.

This study showed that smokers who were interested in trying an e-liquid flavor had a more positive attitude towards e-cigarettes than smokers who were not interested in trying a flavor. In addition, smokers who were interested in trying a flavor considered the social influence 
towards not using e-cigarettes as less strong, which means that the smokers who were not interested in trying an e-cigarette flavor more often find that society thinks that one should not vape. Furthermore, two-third of the smokers reported interest in an e-liquid flavor other than tobacco. This indicates that flavors could support the decision of smokers to switch to vaping ${ }^{57}$, for example by allowing the marketing of e-liquid flavors and other product characteristics that smokers find attractive ${ }^{4}$. The role of e-liquid flavors in supporting both the decisions to switch towards e-cigarette use (for smokers) and to refrain from using e-cigarettes (for never-users) demonstrates the complexity of developing future regulations on e-liquid flavors.

Additional support for the interest in flavors moderating differences in individual factors related to vaping is provided by the different patterns of e-liquid flavors used by dual users and exclusive vapers. In line with previous research, both groups mostly used tobacco and menthol/ mint flavored e-cigarettes at initiation, but exclusive vapers currently used more fruit and sweet e-liquid flavors than dual users $5,34,60-62$. This could be interpreted as vapers switching from tobacco to non-tobacco flavors over time, which is supported by a previous study ${ }^{5}$. Because most adult exclusive vapers included in this study used e-cigarettes for 1 to 5 years and most dual users reported to vape for only less than 6 months (data not shown), it is possible that the dual users may switch to fruit or sweet e-liquid flavors in the future. Longitudinal research is needed to investigate whether and how e-liquid flavors could support dual users in their decision to switch to exclusive vaping. In addition, it would be interesting to investigate the process of e-liquid flavors (or other product characteristics) eventually not living up to dual users' expectation, thereby leaving them to quit vaping and relapse into exclusive cigarette smoking. This information could be used to, for example, stimulate an exchange of knowledge and experiences between exclusive vapers and dual users about the flavored e-liquids they use ${ }^{63}$.

\section{Future research}

Previous studies assessing individual factors related to vaping mostly focused their survey items on e-cigarettes in general. This means that participants are typically asked about their mental representation or beliefs of an unspecified e-cigarette, thereby not taking into account that the e-cigarette is a product that is available in various shapes, sizes, colors, flavors, and more. As our results suggest that flavors may moderate the differences in individual factors related to vaping, we recommend using survey items that represent a specific flavor or other product characteristic. For example, instead of only focusing on perceived susceptibility or attitude towards e-cigarettes in general ${ }^{64,65}$, researchers should also assess perceived susceptibility or attitude towards a specific e-liquid flavor category, such as fruit, candy, or tobacco ${ }^{47}$. In addition, as other product characteristics may moderate differences found in individual factors related to vaping, the impact of for example price, labeling, and packaging of e-cigarettes and e-liquids on individual factors related to vaping should be investigated in different user groups ${ }^{66}$ to determine which characteristics make up their "ideal" e-cigarette. Furthermore, it would be interesting to use sensory research to investigate differences in e-liquid flavor liking between user groups, and how this relates to individual factors related to vaping. Finally, research is needed to investigate the interaction between product characteristics and individual factors related to new and emerging products, such as heated tobacco products and products containing nicotine salts. This will provide insight into which specific product characteristics are most appealing to vulnerable user 
groups, such as never-users and youth, and thus need to be regulated.

\section{Limitations}

Ideally, our sample size would be large enough to stratify our sample into different age groups and different flavor categories. However, our sample size was too small to determine differences in the preference of specific flavor categories between age groups (adults vs. adolescents), and differences in individual factors related vaping between specific flavor categories. In addition, response rates among adolescents was very low, and the rather high education level of participants in this study was not representative of the Dutch population ${ }^{67,68}$. In addition, the sample cannot be considered representative of the Dutch population as oversampling for the smokers and e-cigarette users was performed in order to have sufficient observations. As a result, the percentages of smokers and vapers in our study do not reflect the actual percentages of smokers and vapers in the Dutch population.

\section{Conclusions}

This study demonstrates that being interested in flavors moderates the differences in individual factors related to vaping for never-users and smokers, or vice versa. While the availability of different flavors was reported to be the most attractive product characteristic of e-cigarettes in all user groups, specific flavor preferences varied between never-users, smokers, dual users, and exclusive vapers. Importantly, individual factors related to vaping (knowledge, perceived susceptibility, attitude, social influence, and intention to start vaping) differed between neverusers and/or smokers who were interested in trying an e-liquid flavor and those who did not want to try a flavor. Our results confirm the importance and complexity of regulating e-liquid flavors in a way that both the decision to switch towards vaping (for smokers) and the decision to refrain from vaping (for never-users) are supported. Ideally, regulation should allow marketing of e-liquid flavors that stimulate smokers and dual users to keep or start using e-cigarettes. To make never-users more negative about and keep them from using e-cigarettes, product appeal should be reduced by, for example, restricting the marketing and promotion of e-liquid flavors that they find particularly appealing. 


\section{Appendix}

Appendix Table A6.1: Overview of the included measures to determine user group, demographics, and attractiveness of product characteristics parameters.

\begin{tabular}{|c|c|c|c|c|}
\hline Concept & Item & Answer option & $\begin{array}{l}\text { Explanation of } \\
\text { the concept }\end{array}$ & Reference \\
\hline \multirow[t]{3}{*}{ Type of user } & $\begin{array}{l}\text { A. I smoke or } \\
\text { vape }\end{array}$ & $\begin{array}{l}\text { 1. Both cigarettes and } \\
\text { E-cigarettes } \\
\text { 2. Only cigarettes } \\
\text { 3. Only E-cigarettes } \\
\text { 4. I do not smoke or vape } \\
\text { 5. I have smoked in the past } \\
\text { 6. I have vaped in the past } \\
\text { 7. I have smoked both } \\
\text { cigarettes and vaped in the } \\
\text { past }\end{array}$ & \multirow{3}{*}{$\begin{array}{l}\text { - Response options } \\
\text { A.4 formed the } \\
\text { group never- } \\
\text { users. } \\
\text { - Response options } \\
\text { A.2 \& (B.1 OR } \\
\text { B.2) formed } \\
\text { smokers. } \\
\text { - Response options } \\
\text { A.1 \& (B.1 OR } \\
\text { B.2) \& (C.1 OR } \\
\text { C.2) formed dual } \\
\text { users. } \\
\text { - Response options } \\
\text { A.3 \& (C.1 OR } \\
\text { C.2) formed } \\
\text { e-cigarette users. } \\
\end{array}$} & \multirow{3}{*}{$\begin{array}{l}\text { Pearson, } \\
\text { Hitchman, } \\
\text { Brose, Bauld, } \\
\text { Glasser, } \\
\text { Villanti, } \\
\text { McNeill, } \\
\text { Abrams, Cohen } \\
{ }^{41} \text {; Amato, } \\
\text { Boyle, Levy }{ }^{42} \text {; } \\
\text { International } \\
\text { Tobacco } \\
\text { Control Policy } \\
\text { Evaluation } \\
\text { Project }\end{array}$} \\
\hline & $\begin{array}{l}\text { B. How often do } \\
\text { you smoke? }\end{array}$ & $\begin{array}{l}\text { 1. Every day } \\
\text { 2. Not every day, but at least } \\
\text { once a week } \\
\text { 3. Not every week, but at least } \\
\text { once a month } \\
\text { 4. Less than monthly }\end{array}$ & & \\
\hline & $\begin{array}{l}\text { C. How often do } \\
\text { you vape? }\end{array}$ & $\begin{array}{l}\text { 1. Every day } \\
\text { 2. Not every day, but at least } \\
\text { once a week } \\
\text { 3. Not every week, but at least } \\
\text { once a month } \\
\text { 4. Less than monthly } \\
\text { 5. I have never vaped regularly, } \\
\text { I only tried it once or twice. }\end{array}$ & & \\
\hline \multirow[t]{3}{*}{ Demographics } & $\begin{array}{l}\text { How old are } \\
\text { you? }\end{array}$ & Open question & & \multirow{3}{*}{$\begin{array}{l}\text { Centraal Bureau } \\
\text { voor Statistiek } \\
\text { (CBS) }{ }^{45}\end{array}$} \\
\hline & $\begin{array}{l}\text { What is your } \\
\text { gender? }\end{array}$ & Male-female & & \\
\hline & $\begin{array}{l}\text { What is } \\
\text { your level of } \\
\text { education? }\end{array}$ & $\begin{array}{l}\text { 1. Did not finish school } \\
\text { 2. Primary school to 8th } \\
\text { grade }\end{array}$ & $\begin{array}{l}\text { - Low level of } \\
\text { education: } \\
\text { answer options } 1 \text {, } \\
2,3,4 \text {. } \\
\text { - Middle level } \\
\text { of education: } \\
\text { answer options } \\
5,6 \text {. } \\
\text { - Higher level } \\
\text { of education: } \\
\text { answer options } \\
7-11 .\end{array}$ & \\
\hline
\end{tabular}




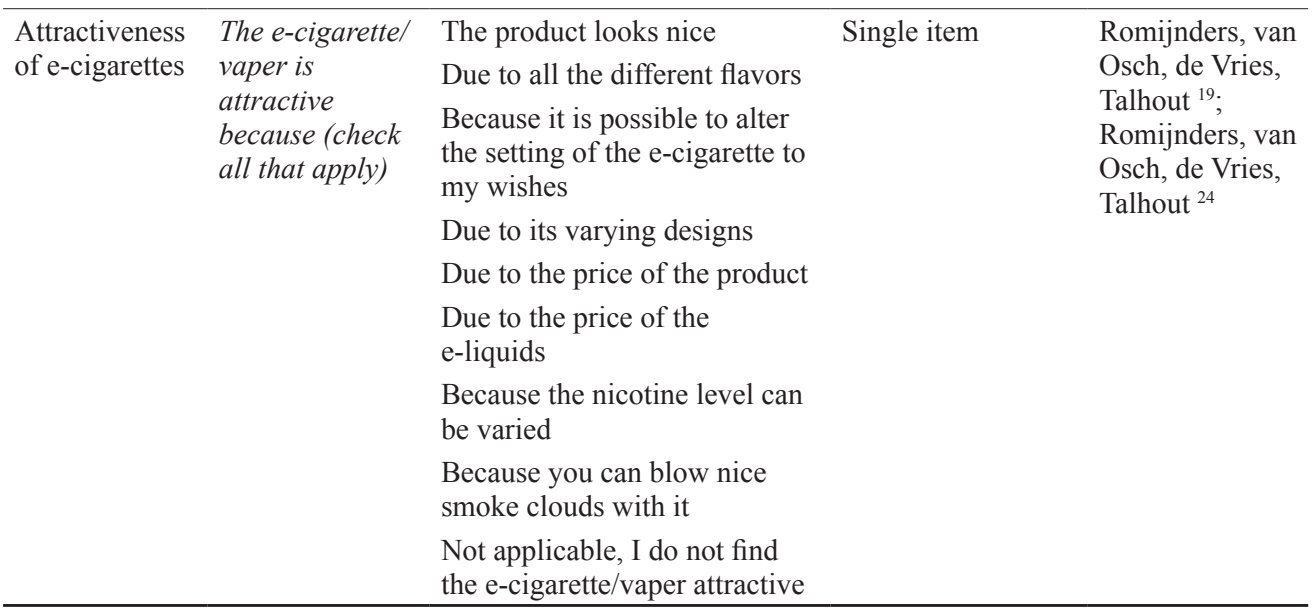


Appendix Table A6.2: Overview of the included measures regarding flavor preferences.

\begin{tabular}{|c|c|c|c|c|}
\hline Concept & Item & Answer option & Explanation of the concept & Reference \\
\hline $\begin{array}{l}\text { Flavor } \\
\text { preference of } \\
\text { never-users } \\
\text { and smokers }\end{array}$ & $\begin{array}{l}\text { If you were } \\
\text { to start using } \\
\text { an e-cigarette, } \\
\text { which flavor } \\
\text { would you like } \\
\text { to try? } \\
\text { (check all that } \\
\text { apply) }\end{array}$ & $\begin{array}{l}\text { - Tobacco } \\
\text { - Menthol/mint } \\
\text { - Nuts } \\
\text { - Herbs, spices } \\
\text { - Coffee/tea } \\
\text { - Cocktails } \\
\text { - Alcohol, other } \\
\text { - Sodas } \\
\text { - Sweet, chocolate } \\
\text { - Sweet, vanilla } \\
\text { - Sweets, other } \\
\text { - Fruit } \\
\text { - Sweet, desserts } \\
\text { - Milk products } \\
\text { - Candy } \\
\text { - Floral } \\
\text { - Unflavored } \\
\text { - None of the flavors }\end{array}$ & \multirow{5}{*}{$\begin{array}{l}\text { For never-users and smokers: if } \\
\text { participants selected "none of the } \\
\text { flavors", no other flavor category } \\
\text { could be selected simultaneously. } \\
\text { These closed answer options were } \\
\text { recoded to the } 13 \text { main categories } \\
\text { of the recently published e-liquid } \\
\text { flavor wheel }{ }^{47} \text {, with the exception } \\
\text { of "none of the flavors". Recoding } \\
\text { the reported flavor preferences } \\
\text { resulted in the following } 13 \text { main } \\
\text { categories: tobacco (survey item: } \\
\text { tobacco), menthol/mint (survey } \\
\text { item: menthol/mint), nuts (survey } \\
\text { item: nuts), spices (survey items: } \\
\text { herbs, spices), coffeeltea (survey } \\
\text { items: coffee; tea), alcohol } \\
\text { (survey items: alcohol, cocktail; } \\
\text { alcohol, other), other beverages } \\
\text { (survey items: soda; sweet, other), } \\
\text { fruit (survey item: fruit), dessert } \\
\text { (survey items: sweet, dessert; } \\
\text { milk product), other sweets } \\
\text { (survey items: sweet, chocolate; } \\
\text { sweet, vanilla), candy (survey } \\
\text { items: sweet, candy), other flavors } \\
\text { (survey items: floral; other) } \\
\text { and unflavored (survey item: } \\
\text { unflavored). } \\
\text { For example, if a never-user or } \\
\text { smoker reported an interest in the } \\
\text { survey items "sweet, candy" and } \\
\text { "alcohol, cocktail", their answers } \\
\text { were recoded as a preference for } \\
\text { the categories candy and alcohol, } \\
\text { respectively. } \\
\text { Open answers from dual and } \\
\text { e-cigarette users were assessed } \\
\text { by two authors (KR and EK) to } \\
\text { support recoding of their closed } \\
\text { answers in accordance with the } \\
\text { categories of the e-liquid flavor } \\
\text { wheel }{ }^{47} \text {. }\end{array}$} & $\begin{array}{l}\text { Yingst, } \\
\text { Veldheer, } \\
\text { Hammett, } \\
\text { Hrabovsky, } \\
\text { Foulds }{ }^{46}\end{array}$ \\
\hline \multirow{4}{*}{$\begin{array}{l}\text { Flavor } \\
\text { preference of } \\
\text { dual users and } \\
\text { e-cigarette } \\
\text { users }\end{array}$} & $\begin{array}{l}\text { Which e-liquid } \\
\text { flavor did you } \\
\text { try first? }\end{array}$ & \multirow{4}{*}{$\begin{array}{l}\text { - Tobacco } \\
\text { - Menthol/mint } \\
\text { - Nuts } \\
\text { - Herbs, spices } \\
\text { - Coffee/tea } \\
\text { - Cocktails } \\
\text { - Alcohol, other } \\
\text { - Sodas } \\
\text { - Sweet, chocolate } \\
\text { - Sweet, vanilla } \\
\text { - Sweets, other } \\
\text { - Fruit } \\
\text { - Sweet, desserts } \\
\text { - Milk products } \\
\text { - Candy } \\
\text { - Floral } \\
\text { - Unflavored }\end{array}$} & & \\
\hline & $\begin{array}{l}\text { Which e-liquid } \\
\text { flavor do you } \\
\text { currently use } \\
\text { most? }\end{array}$ & & & \\
\hline & & & & \\
\hline & & & & \\
\hline
\end{tabular}




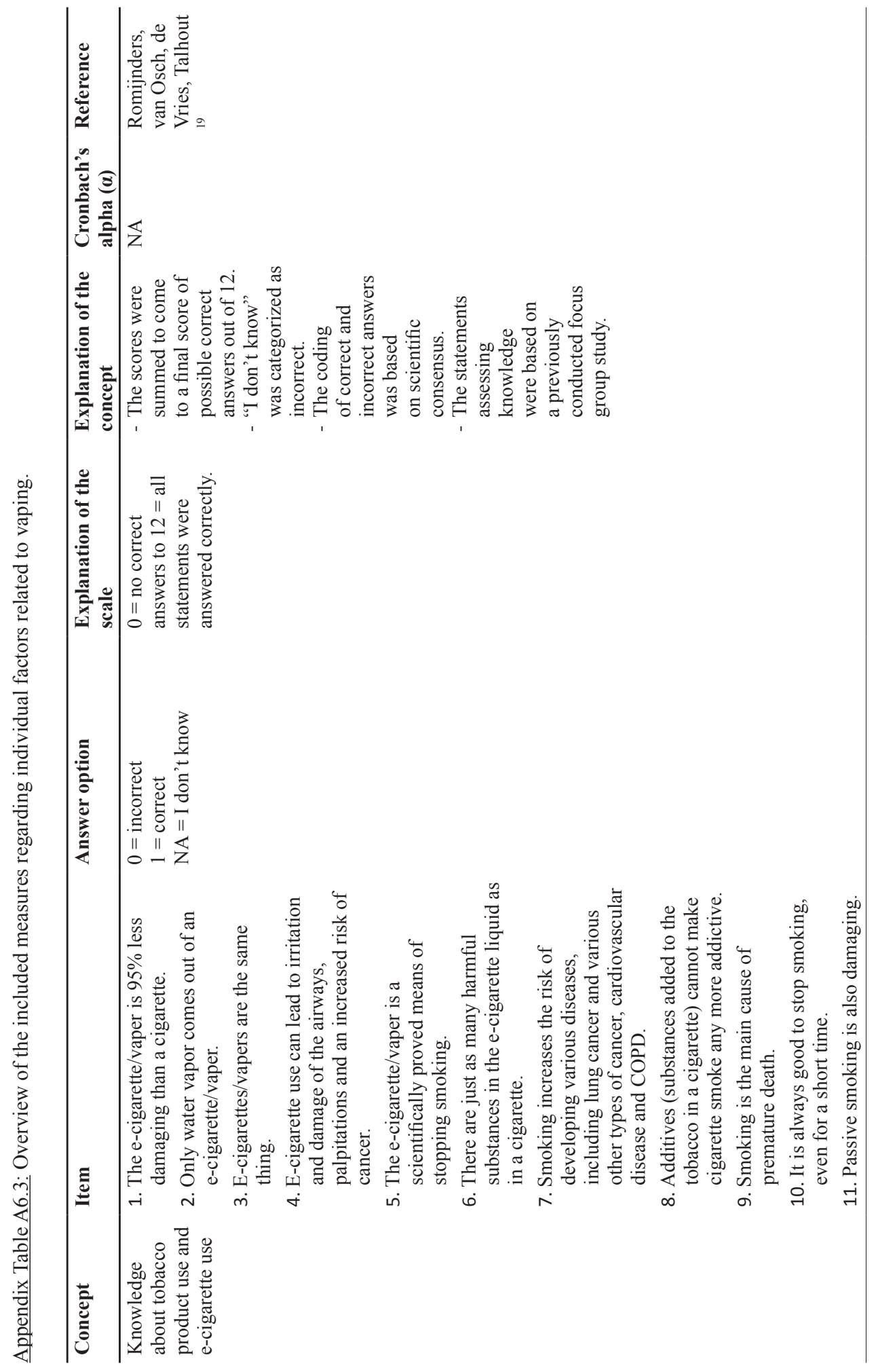




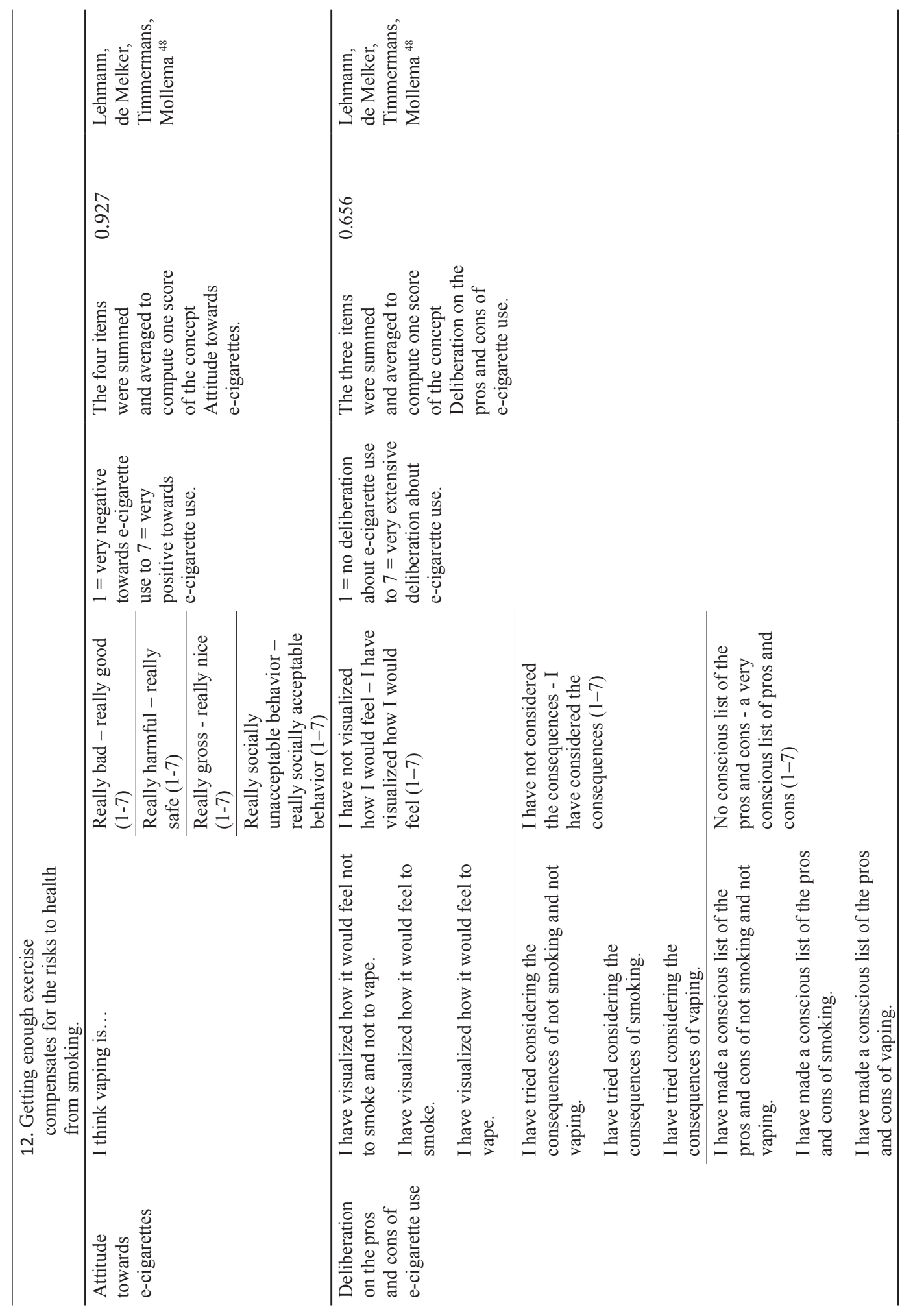




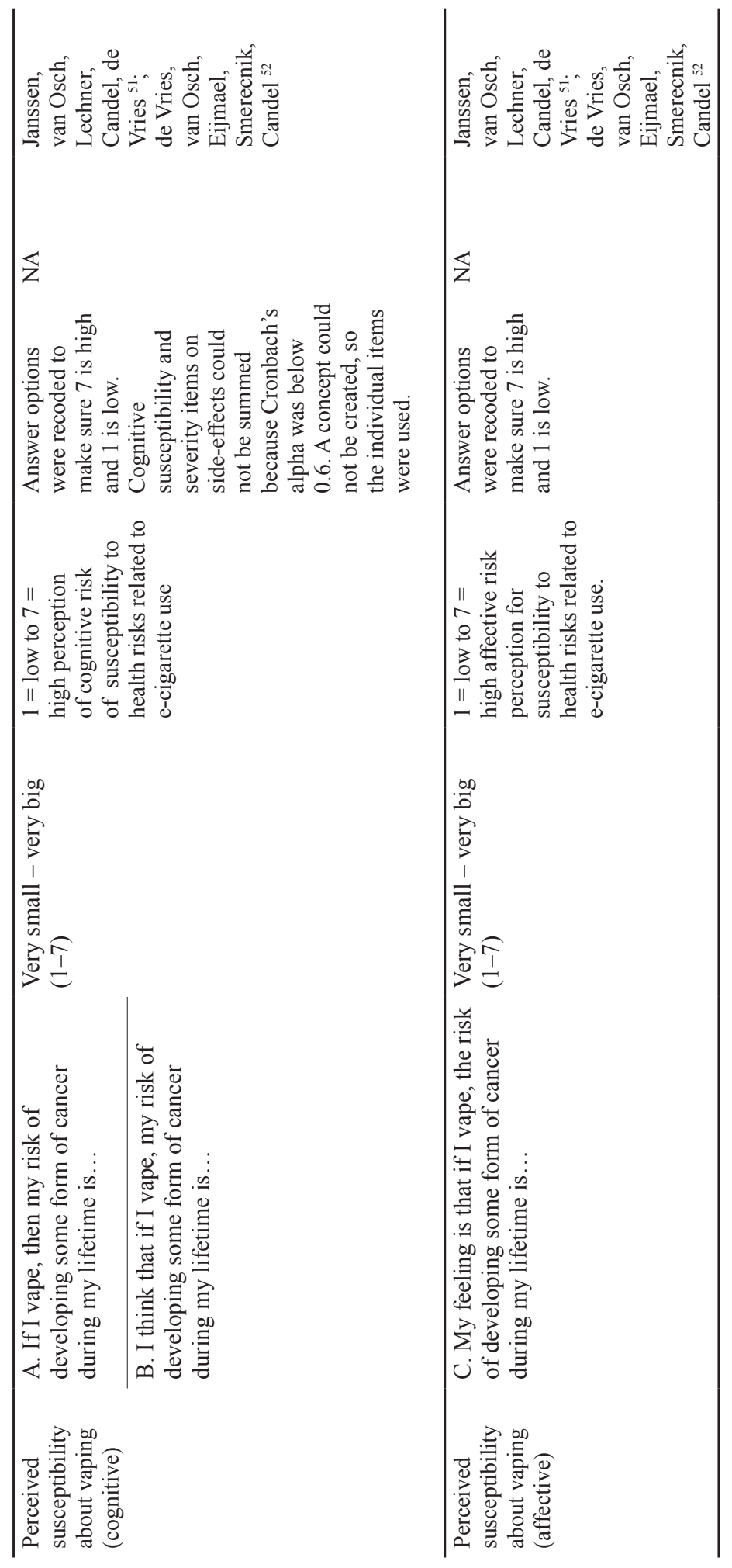




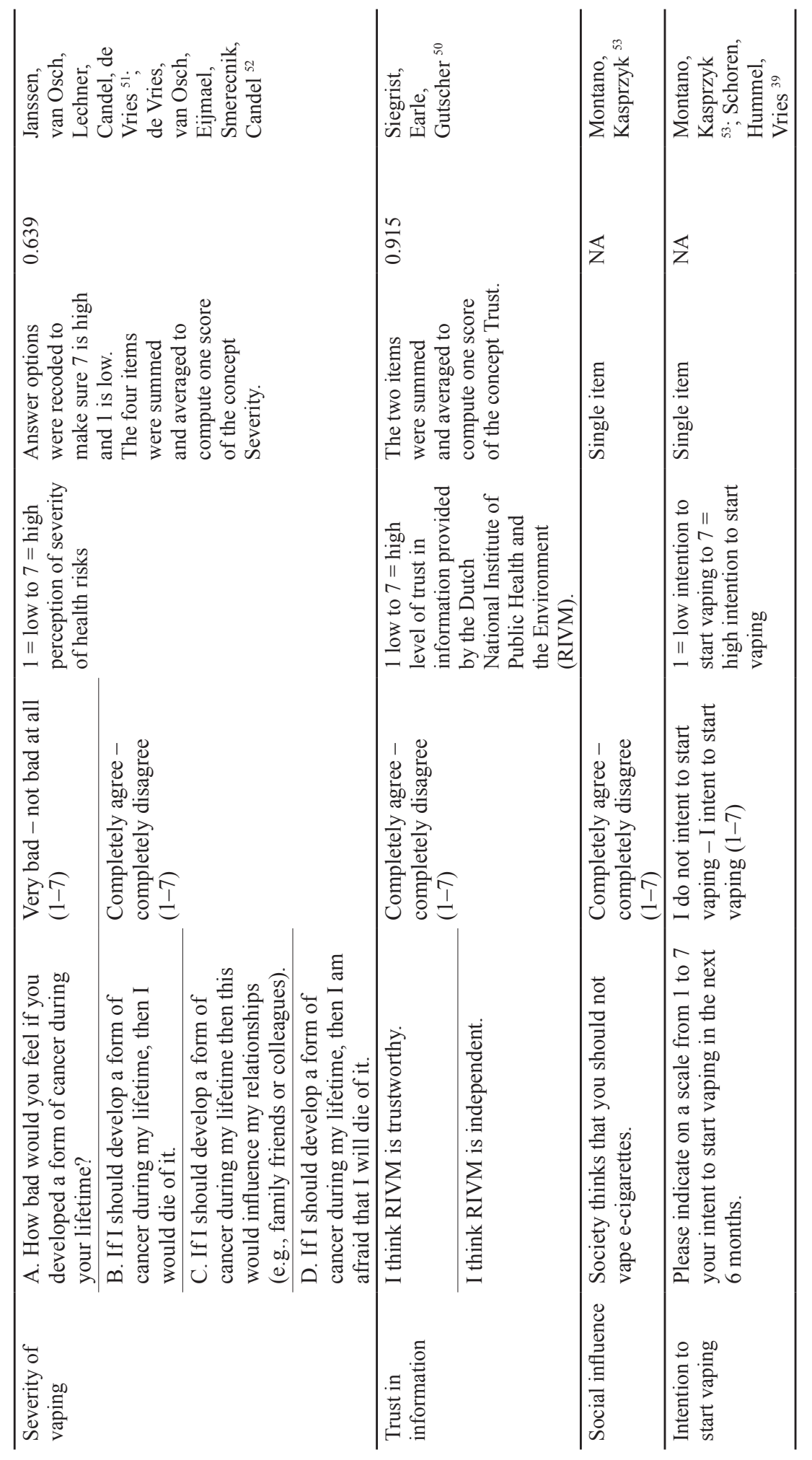




\section{References}

1. Nationaal Expertisecentrum Tabaksontmoediging (Trimbos-instituut). Kerncijfers Roken 2017. 2018; https://www.trimbos.n1/docs/cdade14e-20dc-462a-9f63-1b71ee995a84.pdf.

2. Filippidis FT, Laverty AA, Gerovasili V, Vardavas CI. Two-year trends and predictors of e-cigarette use in 27 European Union member states. Tob Control. 2017;26(1):98-104.

3. McMillen RC, Gottlieb MA, Shaefer RM, Winickoff JP, Klein JD. Trends in Electronic Cigarette Use Among U.S. Adults: Use is Increasing in Both Smokers and Nonsmokers. Nicotine Tob Res. 2015;17(10):1195-1202.

4. Diamantopoulou E, Barbouni A, Merakou K, Lagiou A, Farsalinos K. Patterns of e-cigarette use, biochemically verified smoking status and self-reported changes in health status of a random sample of vapeshops customers in Greece. Intern Emerg Med. 2019;14(6):843-851.

5. Russell C, McKeganey N, Dickson T, Nides M. Changing patterns of first e-cigarette flavor used and current flavors used by 20,836 adult frequent e-cigarette users in the USA. Harm Reduct J. 2018;15(1):33.

6. Nationaal Expertisecentrum Tabaksontmoediging (Trimbos-instituut). Kerncijfers Roken 2017. 2018; https://www.trimbos.nl/docs/cdade14e-20dc-462a-9f63-1b71ee995a84.pdf.

7. Rahman MA, Hann N, Wilson A, Mnatzaganian G, Worrall-Carter L. E-cigarettes and smoking cessation: evidence from a systematic review and meta-analysis. PLoS One. 2015;10(3):e0122544.

8. Chan G, Morphett K, Gartner C, et al. Predicting vaping uptake, vaping frequency and ongoing vaping among daily smokers using longitudinal data from the International Tobacco Control (ITC) Four Country Surveys. Addiction. 2019;114 Suppl 1:61-70.

9. Miech R, Johnston L, O’Malley PM, Bachman JG, Patrick ME. Adolescent Vaping and Nicotine Use in 2017-2018 - U.S. National Estimates. N Engl J Med. 2019;380(2):192-193.

10. Cullen KA, Gentzke AS, Sawdey MD, et al. e-Cigarette Use Among Youth in the United States, 2019. JAMA. 2019;322(21):2095-2103.

11. Prochaska JJ. The public health consequences of e-cigarettes: a review by the National Academies of Sciences. A call for more research, a need for regulatory action. Addiction. 2019;114(4):587-589.

12. Chen J, Bullen C, Dirks K. A Comparative Health Risk Assessment of Electronic Cigarettes and Conventional Cigarettes. Int J Environ Res Public Health. 2017;14(4).

13. Goniewicz ML, Gawron M, Smith DM, Peng M, Jacob P, 3rd, Benowitz NL. Exposure to Nicotine and Selected Toxicants in Cigarette Smokers Who Switched to Electronic Cigarettes: A Longitudinal Within-Subjects Observational Study. Nicotine Tob Res. 2017;19(2):160-167.

14. Levy DT, Borland R, Lindblom EN, et al. Potential deaths averted in USA by replacing cigarettes with e-cigarettes. Tob Control. 2018;27(1):18-25.

15. Kinouani S, Pereira E, Tzourio C. Electronic Cigarette Use in Students and Its Relation with Tobacco-Smoking: A Cross-Sectional Analysis of the i-Share Study. Int J Environ Res Public Health. 2017;14(11).

16. Liu X, Lugo A, Davoli E, et al. Electronic cigarettes in Italy: a tool for harm reduction or a gateway to smoking tobacco? Tob Control. 2020;29(2):148-152.

17. Soneji S, Barrington-Trimis JL, Wills TA, et al. Association Between Initial Use of e-Cigarettes and Subsequent Cigarette Smoking Among Adolescents and Young Adults: A Systematic Review and Meta-analysis. JAMA Pediatr. 2017;171(8):788-797.

18. McRobbie H. Modelling the Population Health Effects of E-Cigarettes Use: Current Data Can Help 
Guide Future Policy Decisions. Nicotine Tob Res. 2017;19(2):131-132.

19. Romijnders K, van Osch L, de Vries H, Talhout R. A Deliberate Choice? Exploring the Decision to Switch from Cigarettes to E-Cigarettes. Int J Environ Res Public Health. 2019;16(4).

20. Marteau TM, Dormandy E, Crockett R. Informed choice: why measuring behaviour is important. Arch Dis Child. 2005;90(5):546-547; author reply 546-547.

21. van den Berg M, Timmermans DR, ten Kate LP, van Vugt JM, van der Wal G. Informed decision making in the context of prenatal screening. Patient Educ Couns. 2006;63(1-2):110-117.

22. Baron J. Thinking and Deciding. 4th ed. Cambridge: Cambridge University Press; 2007.

23. Gray RJ, Hoek J, Edwards R. A qualitative analysis of 'informed choice' among young adult smokers. Tob Control. 2016;25(1):46-51.

24. Romijnders K, van Osch L, de Vries H, Talhout R. Perceptions and Reasons Regarding E-Cigarette Use among Users and Non-Users: A Narrative Literature Review. Int J Environ Res Public Health. $2018 ; 15(6)$.

25. Laverty AA, Vardavas CI, Filippidis FT. Design and marketing features influencing choice of e-cigarettes and tobacco in the EU. Eur J Public Health. 2016;26(5):838-841.

26. Villanti AC, Johnson AL, Ambrose BK, et al. Flavored Tobacco Product Use in Youth and Adults: Findings From the First Wave of the PATH Study (2013-2014). Am J Prev Med. 2017;53(2):139-151.

27. Chen-Sankey JC, Kong G, Choi K. Perceived ease of flavored e-cigarette use and e-cigarette use progression among youth never tobacco users. PLoS One. 2019;14(2):e0212353.

28. McClernon FJ, Pacek LR, Wiley JL. A Conceptual Framework for Understanding Multiple Tobacco Product Use and the Impact of Regulatory Action. Nicotine Tob Res. 2018;21(3):268-277.

29. Zare S, Nemati M, Zheng Y. A systematic review of consumer preference for e-cigarette attributes: Flavor, nicotine strength, and type. PLoS One. 2018;13(3):e0194145.

30. Adriaens K, Van Gucht D, Baeyens F. Differences between Dual Users and Switchers Center around Vaping Behavior and Its Experiences Rather than Beliefs and Attitudes. Int J Environ Res Public Health. 2017;15(1).

31. Shepherd R. Social determinants of food choice. Proc Nutr Soc. 1999;58(4):807-812.

32. Rozin P, Fallon A. The psychological categorization of foods and non-foods: A preliminary taxonomy of food rejections. Appetite. 1980;1(3):193-201.

33. Stafleu A, de Graaf C, Van Staveren WA, Burema J. Affective and cognitive determinants of intention to consume twenty foods that contribute to fat intake. Ecol Food Nutr. 2001;40(3):193-214.

34. Harrell MB, Weaver SR, Loukas A, et al. Flavored e-cigarette use: Characterizing youth, young adult, and adult users. Prev Med Reports. 2017;5:33-40.

35. Huang LL, Baker HM, Meernik C, Ranney LM, Richardson A, Goldstein AO. Impact of nonmenthol flavours in tobacco products on perceptions and use among youth, young adults and adults: a systematic review. Tob Control. 2017;26(6):709-719.

36. Shang C, Huang J, Chaloupka FJ, Emery SL. The impact of flavour, device type and warning messages on youth preferences for electronic nicotine delivery systems: evidence from an online discrete choice experiment. Tob Control. 2018;27(e2):e152-e159.

37. B.V. FIR. Voed de discussie met onderzoek dat zelf nooit ter discussie staat. 2017; https://www. flycatcher.eu/. Accessed 17 August 2018.

38. Romijnders K, Pennings JLA, van Osch L, de Vries H, Talhout R. A Combination of Factors Related to Smoking Behavior, Attractive Product Characteristics, and Socio-Cognitive Factors are Important 
to Distinguish a Dual User from an Exclusive E-Cigarette User. Int J Environ Res Public Health. 2019;16(21).

39. Schoren C, Hummel K, Vries H. Electronic cigarette use: comparing smokers, vapers, and dual users on characteristics and motivational factors. Tob Prev Cessation. 2017;3(April):8.

40. Borland R, Murray K, Gravely S, et al. A new classification system for describing concurrent use of nicotine vaping products alongside cigarettes (so-called 'dual use'): findings from the ITC-4 Country Smoking and Vaping wave 1 Survey. Addiction. 2019;114 Suppl 1:24-34.

41. Pearson JL, Hitchman SC, Brose LS, et al. Recommended core items to assess e-cigarette use in population-based surveys. Tob Control. 2018;27(3):341-346.

42. Amato MS, Boyle RG, Levy D. How to define e-cigarette prevalence? Finding clues in the use frequency distribution. Tob Control. 2016;25(e1):e24-29.

43. International Tobacco Control Policy Evaluation Project. ITC Netherlands (NL11_Pw). International Tobacco Control (ITC). 2017; https://www.itcproject.org/files/ITC_NL11_Replenishment_S_web_ Eng_Dut.pdf. Accessed 10 January 2018.

44. Heatherton TF, Kozlowski LT, Frecker RC, Fagerstrom KO. The Fagerstrom Test for Nicotine Dependence: a revision of the Fagerstrom Tolerance Questionnaire. Br J Addict. 1991;86(9):11191127.

45. Centraal Bureau voor Statistiek (CBS). International Standard Classification of Education (ISCED) [Dutch version]. Heerlen: Centraal Bureau voor Statistiek (CBS); 2018.

46. Yingst JM, Veldheer S, Hammett E, Hrabovsky S, Foulds J. A Method for Classifying User-Reported Electronic Cigarette Liquid Flavors. Nicotine Tob Res. 2017;19(11):1381-1385.

47. Krüsemann EJZ, Boesveldt S, de Graaf K, Talhout R. An E-Liquid Flavor Wheel: A Shared Vocabulary Based on Systematically Reviewing E-Liquid Flavor Classifications in Literature. Nicotine Tob Res. 2018.

48. Lehmann BA, de Melker HE, Timmermans DRM, Mollema L. Informed decision making in the context of childhood immunization. Patient Educ Couns. 2017;100(12):2339-2345.

49. National Institute for Public Health and the Environment (RIVM). Tobacco. 2018; https://www.rivm. nl/tabak. Accessed 19 June 2019.

50. Siegrist M, Earle TC, Gutscher H. Test of a trust and confidence model in the applied context of electromagnetic field (EMF) risks. Risk Anal. 2003;23(4):705-716.

51. Janssen E, van Osch L, Lechner L, Candel M, de Vries H. Thinking versus feeling: differentiating between cognitive and affective components of perceived cancer risk. Psychol Health. 2012;27(7):767783.

52. de Vries H, van Osch L, Eijmael K, Smerecnik C, Candel M. The role of risk perception in explaining parental sunscreen use. Psychol Health. 2012;27(11):1342-1358.

53. Montano DE, Kasprzyk D. Theory of Reasoned Action, Theory of Planned Behavior, and the Integrated Behavioral Model. In: Glanz K, Rimer BK, Viswanath K, eds. Health Behavior and Health Education: Theory, Research and Practice. 4th ed. New Jersey: John Wiley \& Sons; 2008:67-96.

54. ATLAS.ti [computer program]. Version 7.52002-2018.

55. Cullen KA, Ambrose BK, Gentzke AS, Apelberg BJ, Jamal A, King BA. Notes from the Field: Use of Electronic Cigarettes and Any Tobacco Product Among Middle and High School Students - United States, 2011-2018. MMWR Morb Mortal Wkly Rep. 2018;67(45):1276-1277.

56. Chen JC. Flavored E-cigarette Use and Cigarette Smoking Reduction and Cessation-A Large National 
Study among Young Adult Smokers. Subst Use Misuse. 2018;53(12):2017-2031.

57. Buu A, Hu YH, Piper ME, Lin HC. The association between e-cigarette use characteristics and combustible cigarette consumption and dependence symptoms: Results from a national longitudinal study. Addict Behav. 2018;84:69-74.

58. The European Parliament and The Council of the European Union. Directive 2014/40/EU on the approximation of the laws, regulations and administrative provisions of the Member States concerning the manufacture, presentation and sale of tobacco and related products and repealing Directive 2001/37/EC. Vol 2014/40/EU: Official Journal of the European Union; 2014:127.

59. Ministry of Health Welfare and Sports (the Netherlands). Tabaks- en rookwarenwet (Dutch Tobacco Act). 2020; https://wetten.overheid.nl/BWBR0004302/2020-07-01.

60. Schneller LM, Bansal-Travers M, Goniewicz ML, McIntosh S, Ossip D, O’Connor RJ. Use of flavored electronic cigarette refill liquids among adults and youth in the US-Results from Wave 2 of the Population Assessment of Tobacco and Health Study (2014-2015). PLoS One. 2018;13(8):e0202744.

61. O'Connor RJ, Fix BV, McNeill A, et al. Characteristics of nicotine vaping products used by participants in the 2016 ITC Four Country Smoking and Vaping Survey. Addiction. 2019;114 Suppl 1:15-23.

62. Berg CJ. Preferred flavors and reasons for e-cigarette use and discontinued use among never, current, and former smokers. Int J Public Health. 2016;61(2):225-236.

63. Russell C, Dickson T, McKeganey N. Advice From Former-Smoking E-Cigarette Users to Current Smokers on How to Use E-Cigarettes as Part of an Attempt to Quit Smoking. Nicotine Tob Res. 2018;20(8):977-984.

64. Feirman SP, Lock D, Cohen JE, Holtgrave DR, Li T. Flavored Tobacco Products in the United States: A Systematic Review Assessing Use and Attitudes. Nicotine Tob Res. 2016;18(5):739-749.

65. Camenga DR, Fiellin LE, Pendergrass T, Miller E, Pentz MA, Hieftje K. Adolescents' perceptions of flavored tobacco products, including E-cigarettes: A qualitative study to inform FDA tobacco education efforts through videogames. Addict Behav. 2018;82:189-194.

66. Buckell J, Sindelar JL. The impact of flavors, health risks, secondhand smoke and prices on young adults' cigarette and e-cigarette choices: a discrete choice experiment. Addiction. 2019.

67. Centraal Bureau voor Statistiek (CBS). Society: numbers of level of education. Trends in the Netherlands 2018. 2018; https://longreads.cbs.nl/trends18/maatschappij/cijfers/onderwijs/. Accessed 1 March 2019.

68. Van Gelder M, Pijpe AJC. E-epidemiology: a comprehensive update.15(0.007). 



\section{CHAPTER 7}

\section{Sensory evaluation of e-liquid flavors by smelling and vaping yields similar results}

Erna J.Z. Krüsemann

Franziska M. Wenng

Jeroen L.A. Pennings

Kees de Graaf

Reinskje Talhout

Sanne Boesveldt

Published in Nicotine \& Tobacco Research. 2020;22(5): 798-805.

https://doi.org/10.1093/ntr/ntz155. 


\begin{abstract}
Introduction: Sensory research on e-liquid flavors can be performed by means of smelling and vaping. However, data comparing smelling versus vaping e-liquid flavors is lacking. This study aims to investigate if smelling could be an alternative to vaping experiments by determining the correlation for hedonic flavor assessment between orthonasal smelling and vaping of e-liquids, for smokers and non-smokers. Methods: Twenty-four young adult smokers (mean age 24.8 \pm 9.3) and 24 non-smokers (mean age $24.9 \pm 7.7$ ) smelled and vaped 25 e-liquids in various flavors. Participants rated liking, intensity, familiarity, and irritation on a $100-\mathrm{mm}$ Visual Analog Scale. Pearson correlations within and between smelling and vaping were calculated. Differences between user groups were calculated using $t$-tests. Results: Correlation coefficients between smelling and vaping based on mean group ratings were 0.84 for liking, 0.82 for intensity, 0.84 for familiarity, and 0.73 for irritation. Means of the within-subjects correlation coefficients were, respectively, $0.51,0.37,0.47$, and 0.25 . Correlations between smelling and vaping varied across individuals (ranging from -0.27 to 0.87 ) and flavors $(-0.33$ to 0.81 ). Correlations and mean liking ratings did not differ between smokers and non-smokers. Conclusions: The strong group-level correlations between orthonasal smelling and vaping e-liquid flavors justify the use of smelling instead of vaping in future research. For example, smelling could be used to investigate differences in e-liquid flavor liking between (potential) user groups such as nicotinenaïve adolescents. The more modest within-subject correlations and variation across individuals and flavors merit caution in using smelling instead of vaping in other types of experiments. Implications: This study supports the use of orthonasal smelling (instead of vaping) e-liquids to measure hedonic flavor perception in some studies where vaping would be inappropriate or not feasible. Examples of research situations where smelling e-liquids may be sufficient are (1) investigating nicotine-naïve individuals (i.e., non-users), (2) investigating individuals under legal age for e-cigarette use (i.e., youth and adolescents), (3) investigating brain responses to exposure of e-liquid flavors using functional magnetic resonance imaging or electroencephalography, and (4) comparing hedonic flavor assessment between adolescent non-users and current smokers to provide support for future regulations on e-liquid flavors.
\end{abstract}




\section{Introduction}

The use of electronic cigarettes (e-cigarettes) has become increasingly popular over the past years ${ }^{1,2}$. Literature describes the variety of flavors being an important reason for e-cigarette use ${ }^{3,4}$. Not surprisingly, the number and variety of flavors on the e-cigarette market has exploded ${ }^{5}$, for example, up to 245 unique flavors in the Netherlands in $2017^{6}$. While most e-cigarette users are concurrent or former smokers ${ }^{7-9}$, the availability of appealing flavors may also stimulate e-cigarette use among non-smokers and adolescents ${ }^{10-14}$.

As e-cigarettes are less harmful than cigarettes ${ }^{15-19}$, smokers' health may benefit from using e-cigarettes compared to smoking combustible tobacco. However, as e-cigarettes are not safe, use among current non-users and adolescents should be prevented ${ }^{15,20}$. Research showed that flavor use and preferences may differ between user groups ${ }^{9,10,21-23}$. Thus, e-liquid flavors could be regulated in order to maximize public health benefits, and for this, research on flavor preferences among different user groups is needed.

Flavor perception is a combined sensation of olfactory stimuli (smell), gustatory stimuli (taste), and chemesthesis (touch) ${ }^{24,25}$. Examples of chemesthetic sensations in the mouth are the burn of capsaicinoids in chili peppers and the cooling of menthol ${ }^{26}$. Sensory flavor research can be conducted by means of smelling and tasting, that is, smelling and vaping when investigating e-liquid flavors. Smelling and vaping reflect two different ways of olfactory assessment: orthonasally, where ambient odors enter via the nose when we sniff, and retronasally, caused by the airflow from the back of the mouth and throat to the nose when we eat and swallow (similar to vaping). So far, sensory research on e-liquid perception is limited to a few vaping experiments 27-29. Whereas orthonasal smelling experiments only focus on the olfactory component of flavor perception, vaping evokes olfactory, gustatory, as well as chemesthetic sensations. However, although reflecting real consumer behavior, vaping experiments are associated with two important ethical restrictions regarding the study population. That is, participants are required to be over 18 years old, and, when investigating nicotine-containing e-liquids, should be experienced vapers or smokers because of the addictive effect of nicotine ${ }^{30}$. These restrictions do not apply to smelling experiments, which thus provide the opportunity to investigate adolescents and nonusers. In addition, experiments based on smelling e-liquid flavors are faster and less expensive than vaping experiments, because they do not require the use of e-cigarettes. While orthonasal smelling experiments are a potential alternative approach for sensory research on e-liquids, sensory comparability of smelling and vaping data is yet unknown.

Previous research on food and beverages finds comparable results between orthonasal and retronasal perception. For example, studies on wine and Pisco spirits found comparable ratings between orthonasal (sniffing) and retronasal olfaction (sipping and swallow) in terms of descriptive profiling using trained panelists ${ }^{31,32}$. Furthermore, although neural responses seem to differ, it was shown that pleasantness ratings were comparable between orthonasal and retronasal presentation of chocolate odor ${ }^{33}$. Another study found that liking, sweetness, and intensity of e-liquid flavors are primarily driven by the e-liquids' volatile compounds, indicating that e-liquid flavor perception more strongly depends on (retronasal) olfaction than on taste ${ }^{34}$. In line with this, we hypothesize that hedonic assessment of e-liquids by means of orthonasal smelling and vaping is comparable, and, thus, that smelling experiments could be used to replace vaping 
experiments.

To test this hypothesis, this study aims to investigate if hedonic evaluation of e-liquid flavors by orthonasal smelling is correlated with (retronasal) vaping ratings. In addition, the correlation between smelling and vaping will be determined for intensity, familiarity, and irritation, as these factors are known to influence liking ${ }^{29,35,36}$. As smokers are used to inhalation, flavor perception through vaping may differ between smokers and non-smokers. Therefore, we also investigate whether there are differences between smokers and non-smokers. 


\section{Methods}

\section{Participants}

Participants were recruited from Wageningen and surroundings (the Netherlands) by E-mail, social media, flyers, and word-of-mouth. Twenty-four smokers ( $50 \%$ female; mean age $=24.8 \pm$ 9.3, range 18-54 years old) and 24 non-smokers (50\% female; mean age $=24.9 \pm 7.7$, range 20 55 years old) were included. Smokers reported to smoke more than 1 cigarette/day on average (mean $=10.2 \pm 6.5$ cigarettes/day) and not only in the weekends. Non-smokers were required to have never smoked or have quit smoking for $>12$ months. Panel characteristics are shown in Appendix Table A7.1. Sample size ( $n=24$ per group, accounting for potential dropout) was determined using a statistical algorithm with 1000 random samplings of a subset of the study population from preliminary smelling experiments (data not shown), and aimed at identifying a correlation coefficient of at least $0.25-0.45$ (based on a correlation between liking and familiarity in the preliminary smelling experiments, as well as on correlations between liking and sweetness, coolness, harshness, and bitterness in previous literature ${ }^{29}$ ), with more than $95 \%$ power and significant at $p<0.01$.

Participants were screened using a self-report questionnaire to: be between 18 and 55 years of age; be healthy; never have used an e-cigarette before; and have a good proficiency of the Dutch language. In addition, participants had to have normal olfactory function according to the Sniffin' Sticks identification test ${ }^{37}$. Exclusion criteria were: pregnancy or lactating; allergies for any of the product flavors under investigation in this study; employment at the Division of Human Nutrition and Health of Wageningen University; and participation in other medicalscientific research.

Participants who completed the study received a financial compensation; participants who did not pass the screening test received a gift voucher. All participants provided written informed consent at their first visit. The study was approved by the Medical Ethical Committee of Wageningen University (NL65748.081.18).

\section{Experimental procedure}

Eligible participants were invited for a screening session to determine their olfactory functioning. If they passed the olfactory test ( $\geq 12$ correct answers out of 16) ${ }^{37}$, they were familiarized with the Visual Analog Scale (VAS) and the type of e-cigarette used in this study, by taking a maximum of five puffs and rating liking (how much do you like this flavor?). The e-cigarette contained a nicotine-free, unflavored e-liquid. Participants decided themselves whether to inhale the vape over their lungs or to directly exhale the vape from their mouth, as long as they did this consistently over all sessions (see Appendix Table A7.1).

Test sessions took place in sensory booths, each equipped with a computer, water tap, and tissues. The room was accommodated with a controlled ventilation system of five air changes per hour. Participants were asked to refrain from using scented perfumes on test days, and from smoking, chewing gum, brushing their teeth, and eating or drinking anything apart from water at least 1 hour prior to the test sessions. Two smelling and two vaping sessions were scheduled during which participants assessed 25 e-liquid flavors in total (13 and 12 e-liquids per session) on liking, intensity, familiarity, and irritation. The order of the sessions was counterbalanced 
across individuals. The time between two sessions was at least 1 week.

VAS ratings were collected using EyeQuestion software V.4.11.19 (Logic8 BV). During the test sessions, participants were asked to take one puff (vaping sessions) or to smell the e-liquid sample once (smelling sessions). Between each product, a break of at least 30 seconds was installed to prevent olfactory adaptation. Within each session, the product sequence was randomized. All products were first assessed on liking ("how much do you like this odor/ flavor?"). Subsequently, after a 1-minute break, products were assessed on perceived intensity ("how strong do you perceive this odor/flavor?"), familiarity ("how familiar are you with this odor/flavor?"), and irritation ("to what extent does this odor/flavor give you an irritating feeling in your nose/mouth or throat?"). Participants were explicitly asked to only focus on the odor/ flavor instead of on overall (vaping) experience. Participants were allowed to rinse their mouth with water between each sample. For hygienic purposes, fresh mouthpieces were used every time a participant assessed a new flavor. No adverse events occurred and all measures and conditions have been reported.

\section{Materials and equipment}

During the training and test sessions, a 100-mm VAS was used to assess liking, intensity, familiarity, and irritation (left anchor at $10 \mathrm{~mm}$ : "Not at all", right anchor at $90 \mathrm{~mm}$ : "Extremely"). Twenty-five commercial e-liquids, from four different brands, were purchased from three online shops. E-liquids contained a base of 70\% propylene glycol (PG) and 30\% vegetable glycerin $(\mathrm{VG})$, and $0 \mathrm{mg} / \mathrm{mL}$ nicotine. In order to obtain a high variety of flavors, selection of e-liquid flavors was based on the different categories of our recently published e-liquid flavor wheel ${ }^{38}$ : tobacco (American tobacco with hazelnut, Indonesian tobacco, and Oriental tobacco); menthol/ mint (mint and peppermint); nuts (hazelnut); spices (fennel and licorice); coffee/tea (coffee and cappuccino); alcohol (piña colada and whiskey); other beverages (cola and energy drink); fruit (strawberry, lemon, banana, and watermelon); dessert (cookie); candy (cotton candy and red candy); other sweets (caramel, chocolate, and vanilla); and unflavored (PG/VG base). For vaping, eGo-type e-cigarettes were used with a battery capacity of $900 \mathrm{mAh}$, constant voltage, and a coil resistance of $2 \mathrm{Ohm}$.

\section{Sample preparation}

For smelling, 10 drops of an e-liquid were put in a 50-mL brown glass vial. For vaping, e-cigarette clearomizers were filled with sufficient e-liquid (with a maximum of $1.6 \mathrm{~mL}$ ) and covered in tin foil to avoid visual cues. E-cigarettes and vials were labelled with a random three-digit code. Vials and e-cigarettes were cleaned and filled with e-liquid up to two days before each test session. The coil was replaced when cleaning the e-cigarettes. Every other week, a new set of e-liquids from the same batch was used. E-liquids were stored within their original package at room temperature. Vials and e-cigarettes filled with e-liquid were stored in the dark at room temperature. 


\section{Data analysis}

Data were analyzed using R statistical software V.3.5.1. No data were excluded. Results are presented for the whole group and separately for smokers and non-smokers.

\section{Mean ratings}

The mean score and standard error over all flavors were calculated for each variable, for both smelling and vaping, separately for smokers and non-smokers. A constant value of 50 was subtracted in order to center ratings around zero. The effect of assessment type (smelling vs. vaping) and smoking status (smokers vs. non-smokers) and their interaction were examined using a 2-way ANOVA model. The model included the participant as a covariate to allow for repeated (paired) measurements per individual. To correct for multiple testing, Benjamini-Hochberg false discovery rate (FDR) ${ }^{39}$ adjusted $p$-values of less than 5\% were considered significant.

Next, for each flavor separately, mean scores and standard errors were calculated for each variable. For each flavor, differences between smelling and vaping were compared using paired $t$-tests; smokers were compared to non-smokers for liking using an unpaired $t$-test. To correct for multiple testing, Benjamini-Hochberg false discovery rate ${ }^{39}$ adjusted $p$-values of less than $5 \%$ were considered significant.

\section{Correlation coefficients}

Since the correlations between smelling and vaping for each variable depend on data that can be assigned to participants $(n=48)$ as well as flavors $(n=25){ }^{40}$, correlations were calculated in two different ways. First, for each variable (liking, intensity, familiarity, irritation), Pearson correlation coefficients between smelling and vaping were calculated for the mean ratings per flavor, thus based on 25 pairs of data. This was done for the whole group, and separately for smokers and non-smokers. To determine if correlations for smokers and non-smokers were significantly different from zero and from each other $(p \leq 0.01)$, a Fisher's Z-transformation was applied in order to transform the sampling distribution of the Pearson correlations towards a normal distribution. Transformed correlations were compared using an unpaired $t$-test, and $p$-values $\leq 0.01$ were considered significant.

Secondly, to allow insight into individual participants, a Pearson correlation was calculated for each individual (i.e., within-subjects correlations) for liking, intensity, familiarity, and irritation ratings. These individual correlations were Fisher's Z-transformed, and the overall average was calculated and back-transformed. This was done for the whole group, and separately for smokers and non-smokers. Unpaired $t$-tests on Fisher's Z-transformed correlations were used to determine if correlations for smokers and non-smokers were significantly different from zero and from each other $(p \leq 0.01)$. In addition, to examine variability across flavors, a Pearson correlation between smelling and vaping was calculated for each of the 25 e-liquid flavors separately.

Finally, within smelling and vaping data, we calculated Pearson correlation coefficients between the four variables (using ratings across all participants and flavors). $T$-tests were used to determine if correlations were significantly different from zero $(p \leq 0.05)$. 


\section{Results}

\section{Mean liking, intensity, familiarity, irritation ratings of e-liquid flavors}

Mean ratings for liking, familiarity, and irritation showed no significant effects for assessment type, smoking status, and their interaction (see Table 7.1). A significant interaction term was found for intensity ( $p=0.01$ after false discovery rate correction). Intensity ratings were higher for smelling compared to vaping (for both user groups), and non-smokers rated the flavors as more intense compared to smokers (for both assessment types). The significant interaction reflects particularly low intensity ratings for assessment by means of vaping in smokers.

For the individual e-liquid flavors, mean liking ratings did not significantly differ between smokers and non-smokers. Furthermore, there were no significant differences between smokers and non-smokers regarding intensity, familiarity, and irritation (except vaping of caramel, which scored significantly higher in intensity for non-smokers compared to smokers; $p=0.04$ after false discovery rate correction). Information on mean ratings for liking, intensity, familiarity, and irritation for smelling and vaping of individual flavors can be found in Appendix A7.2 and Appendix A7.3.

Table 7.1: Group means ( $\pm \mathrm{SE}$ ) and two-way ANOVA $p$-values for liking, intensity, familiarity, and irritation ratings.

\begin{tabular}{|c|c|c|c|c|c|c|c|}
\hline & \multicolumn{2}{|c|}{ Smokers $(n=24)$} & \multicolumn{2}{|c|}{ Non-smokers $(\mathrm{n}=24)$} & \multicolumn{3}{|c|}{$\begin{array}{c}\text { 2-way ANOVA } \\
\text { (FDR corrected p-values) }\end{array}$} \\
\hline & Smelling & Vaping & Smelling & Vaping & $\begin{array}{l}\text { Assessment } \\
\text { type }\end{array}$ & $\begin{array}{l}\text { Smoking } \\
\text { status }\end{array}$ & Interaction \\
\hline Liking & $-1.4 \pm 1.0$ & $0.8 \pm 0.9$ & $1.2 \pm 0.9$ & $-0.1 \pm 0.9$ & 0.39 & 0.17 & 0.17 \\
\hline Intensity & $9.6 \pm 0.8$ & $3.5 \pm 0.8$ & $12.1 \pm 0.8$ & $11.4 \pm 0.8$ & 0.56 & 0.17 & $0.01 *$ \\
\hline Familiarity & $7.0 \pm 1.0$ & $2.5 \pm 1.1$ & $4.8 \pm 1.0$ & $2.1 \pm 1.1$ & 0.17 & 0.23 & 0.43 \\
\hline Irritation & $-22.1 \pm 0.9$ & $-26.2 \pm 0.8$ & $-23.5 \pm 0.8$ & $-25.5 \pm 0.8$ & 0.17 & 0.27 & 0.27 \\
\hline
\end{tabular}

The ANOVA model included assessment type (smelling vs. vaping) and smoking status (smokers vs. nonsmokers). Data were collected on a 100-mm Visual Analog Scale (anchored "not at all" to "extremely") and centered around zero by subtracting a constant value of 50 .

* Significant $(p \leq 0.05)$ after FDR correction.

FDR, false discovery rate. 


\section{Correlations between smelling and vaping}

The correlation coefficient between smelling and vaping for liking, based on mean group ratings, was 0.84 . Figure 7.1 shows the correlation coefficients based on the mean smelling and vaping ratings of the whole group for all variables. The correlation coefficients separately for smokers and non-smokers can be found in Appendix A7.4.

The mean of the within-subject correlations between smelling and vaping for liking was 0.51 . Table 7.2 shows the means of the within-subject correlation coefficients for all variables. For each variable, correlations were significantly different from zero $(p \leq 0.01)$. None of the correlations differed significantly between smokers and non-smokers. The correlations for liking separated by flavor and participant can be found in Appendix A7.5.

Table 7.2: Mean of the 48 within-subjects correlation coefficients between smelling and vaping for liking, intensity, familiarity, and irritation, for the whole group $(\mathrm{n}=48)$ and separately for smokers $(\mathrm{n}=24)$ and non-smokers $(\mathrm{n}=24)$.

\begin{tabular}{lllll}
\hline & Whole group & Smokers & Non-smokers & p-value* \\
\hline Liking & 0.51 & 0.49 & 0.54 & 0.475 \\
Intensity & 0.37 & 0.34 & 0.40 & 0.280 \\
Familiarity & 0.47 & 0.44 & 0.50 & 0.310 \\
Irritation & 0.25 & 0.21 & 0.29 & 0.239 \\
\hline
\end{tabular}

The same data are presented as correlation coefficients based on mean group ratings in Figure 7.1.

* Correlations between smokers and non-smokers were considered significantly different if $p \leq 0.01$. 

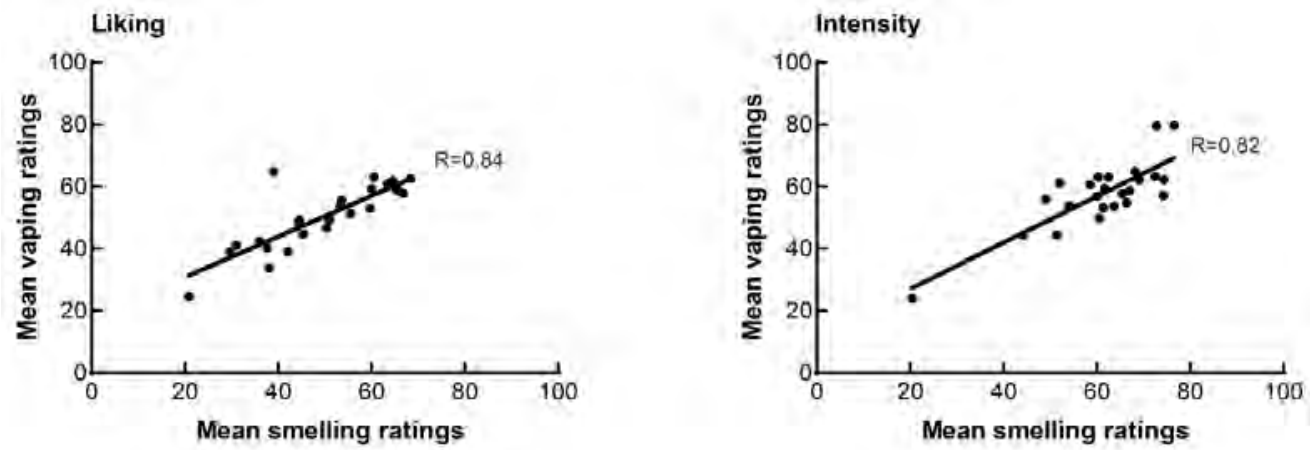

Familiarity
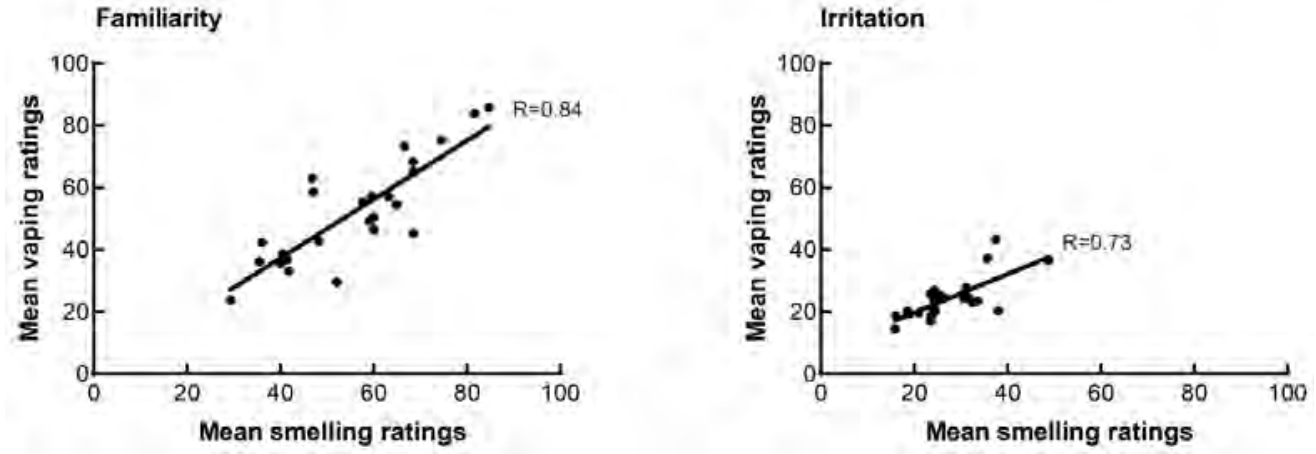

Figure 7.1: Correlation coefficients between smelling and vaping based on the mean group ratings of each of the 25 products, for liking (top left), intensity (top right), familiarity (bottom left), and irritation (bottom right). Each dot represents the mean group rating for a product on a 100-mm Visual Analog Scale. The same data are presented as mean of within-subject correlation coefficients in Table 7.2. 


\section{Correlations within smelling and vaping}

Table 7.3 shows the correlation coefficients between ratings for liking, intensity, familiarity, and irritation over the whole group and all flavors. For both smelling and vaping, significant correlations were found between liking and familiarity $(R=0.45$ and $R=0.37$, for smelling and vaping, respectively), liking and irritation $(\mathrm{R}=-0.29$ and $R=-0.16)$, intensity and familiarity $(R$ $=0.34$ and $R=0.36)$, and between intensity and irritation $(R=0.35$ and $R=0.29)$. In addition, for vaping, significant correlations were found between liking and intensity $(R=-0.07)$ and between familiarity and irritation $(R=0.08)$.

Table 7.3: Pearson correlation coefficients between liking, intensity, familiarity, and irritation, for smelling and vaping, for smokers and non-smokers combined.

\begin{tabular}{lllllllll}
\hline \multicolumn{3}{l}{ Smelling } & \multicolumn{7}{c}{ Vaping } \\
\hline & Liking & Intensity & Familiarity & Irritation & Liking & Intensity & Familiarity & Irritation \\
\cline { 2 - 8 } Liking & 1.00 & -0.04 & $0.45^{*}$ & $-0.29^{*}$ & 1.00 & $-0.07^{*}$ & $0.37^{*}$ & $-0.16^{*}$ \\
Intensity & & 1.00 & $0.34^{*}$ & $0.35^{*}$ & & 1.00 & $0.36^{*}$ & $0.29^{*}$ \\
Familiarity & & 1.00 & 0.02 & & & 1.00 & $0.08^{*}$ \\
Irritation & & & 1.00 & & & & 1.00 \\
\hline
\end{tabular}

* Significantly different from zero $(p \leq 0.05)$. 


\section{Discussion}

This study aimed to investigate if hedonic evaluation of e-liquid flavors by orthonasal smelling is correlated with (retronasal) vaping ratings. We found strong positive group-level correlations and more modest within-subject correlations between smelling and vaping for ratings of liking, intensity, familiarity, and irritation of e-liquid flavors. Correlations between smelling and vaping varied across individuals and flavors, but did not differ between smokers and non-smokers.

The strong positive correlations between smelling and vaping are in line with previous studies that found comparable results between orthonasal and retronasal perception of food products ${ }^{31-33,41,42}$. This can be explained by physiological reasons, as both orthonasal (smelling) and retronasal smell (vaping) cause the volatile flavor molecules to be sensed by the same olfactory receptors located in the nasal epithelium. The strong group-level correlations between smelling and vaping justify the use of orthonasal smelling (instead of vaping) e-liquids to measure hedonic flavor perception in studies where vaping would be inappropriate or not feasible. Examples of such research situations are investigating nicotine-naïve individuals (i.e., non-users) or individuals under legal age for e-cigarette use (i.e., youth and adolescents). In addition, smelling can be used to compare hedonic flavor assessment between adolescent nonusers and current smokers, providing support for future regulations on e-liquid flavors. Finally, neural responses to e-liquid flavor/odor exposure (e.g., using functional magnetic resonance imaging or electroencephalography) can help to better understand the role of flavors in liking of and reward from e-cigarettes.

This study showed that the correlations between smelling and vaping varied across flavors. The correlation between smelling and vaping for liking of whiskey flavor was negative, potentially because the whiskey-flavored e-liquid received the lowest ratings for liking. The positive correlations for other flavors varied from modest to strong (see Appendix Table A7.5). As we used only one or two e-liquids to represent a main flavor category, the across-flavors variability in correlation coefficients could be assigned to the individual products selected rather than to flavor categories in general. Consequently, smelling experiments can be used in the future to investigate overall flavor liking among different user groups. However, in order to investigate differences between flavor categories or even between individual flavors, each category or individual flavor should be represented by multiple e-liquids from various subcategories (e.g., e-liquids with a mojito, beer, and rum flavor to represent the "alcohol" category) or brands (e.g., multiple strawberry- or orange-flavored e-liquids from different brands).

\section{Correlations within smelling and vaping}

The correlations within smelling and vaping showed that liking was positively correlated with familiarity and negatively with irritation, which is in line with previous literature ${ }^{29,36}$. There was no correlation for smelling between liking and intensity, and a low negative correlation for vaping (i.e., the higher the intensity ratings, the less a flavor was liked). This could be explained by the typically non-linear, inverted " $U$ " shaped relation between intensity and liking, where liking first increases with physical (or sensory) intensity, peaks, and then decreases ${ }^{43}$. Since commercial e-liquids were used, it may be assumed that the flavors were designed to have an intensity that results in optimal liking ratings (peak of the curve). Following the inverted "U" 
shaped curve, higher intensity ratings would then indeed result in lower ratings for liking. This is supported by, for instance, outcomes for the strawberry-flavored e-liquid: for smelling, mean intensity ratings were much higher and, consequently, mean liking ratings were much lower than for vaping.

In addition, this study found higher intensity ratings for smelling than for vaping. In line with this, previous research showed that odors presented in an orthonasal way were rated as more intense than odors administered in a retronasal way ${ }^{41,42}$. An explanation may be that odors are typically encountered at higher concentrations during orthonasal perception of liquids versus retronasal perception ${ }^{41,44}$. Therefore, it should be taken into account that orthonasal compared to retronasal presentation of e-liquid flavors may require lower concentrations to produce the same intensity when replacing future vaping experiments by smelling experiments. Future research is needed to determine an optimal and consistent e-liquid intensity for conducting smelling experiments. A possible approach may be heating the e-liquids, as increasing temperature may change flavor perception due to an increased release of volatile molecules ${ }^{45,46}$.

\section{Comparing smokers and non-smokers}

This study found that smokers perceived the flavors as less intense than non-smokers did; intensity ratings were particularly low for assessment by means of vaping in smokers. Although smokers are more prone to olfactory dysfunction than non-smokers ${ }^{47}$, we only included participants with normal olfactory function in our study. However, smokers may have rated intensity during vaping lower because they are used to inhale smoke.

In addition, we found that the flavors rated highest and lowest in liking differed between smokers and non-smokers (see Appendix Table A7.3). Whereas liking was highest for mint and peppermint among smokers, sweet flavors such as strawberry, watermelon, and caramel scored highest for liking among non-smokers. Although this may suggest a trend that is in line with previous literature ${ }^{13}$, differences in flavor liking between smokers and non-smokers were not significant. A reason for this may be that we asked participants to focus on flavor perception only ("how much do you like this flavor?") rather than creating an e-cigarette context (e.g., "how much would you like to try an e-cigarette with this flavor?"). Because liking depends on context factors ${ }^{43}$ and flavor preference in an e-cigarette context may differ between user groups ${ }^{9,10,21-23}$, differences in hedonic flavor assessment between user groups may have been found if questions were to be asked in an e-cigarette context. In addition, as people are often unable to identify unlabeled flavors without a predefined list of verbal descriptors to choose from ${ }^{48}$ and learned associations from previous experiences can influence the hedonic perception ${ }^{49}$, outcomes may have been different if participants would be aware of the specific flavors used in this study. Overall, our study design was chosen to determine the correlation between smelling and vaping for liking of e-liquid flavors. Future research investigating differences in flavor liking between user groups, for example using smelling experiments, would benefit from creating an e-cigarette context and labeling the flavors under investigation.

\section{Strengths of this study}

A strength of this study was that we included participants who had never used an e-cigarette; thus, outcomes were not influenced by prior vaping experiences. In addition, the panel consisted of a 
balanced number of smokers and non-smokers (50\% were smokers), and both user groups had a similar mean age and equal male/female ratio (50\% were male). Finally, we selected e-liquid flavors from all different flavor categories ${ }^{38}$ and covered a wide hedonic range in order to rule out strong influences from individual flavors on the overall correlations.

\section{Limitations and future directions}

The more modest within-subject correlations, variation across individuals, and variation across specific e-liquid flavors found in this study suggest that future research is needed to investigate whether the use of smelling instead of vaping is applicable to other research situations. First, this study used nicotine-free e-liquids (for ethical reasons), but the use of nicotine-containing e-liquids may have resulted in different outcomes. That is, nicotine may be expected to evoke taste and chemesthetic sensations during vaping (i.e., bitterness and harshness) that contribute to flavor (dis)liking. As these sensations cannot be perceived by means of orthonasal smelling, a research situation that includes nicotine-containing e-liquids may yield lower correlations between hedonic smelling and vaping ratings. In addition, nicotine may cause participants to have more difficulties restricting their ratings to odor/flavor perception without being influenced by the overall vaping experience Future studies are thus necessary to determine the degree to which smelling and vaping ratings align when using nicotine-containing e-liquids.

Second, even though previous literature showed that e-liquid flavor perception more strongly depends on (retronasal) olfaction than on taste ${ }^{34}$, it would be interesting to investigate the role of taste in orthonasal assessment of e-liquid flavors (e.g., via learned associations). Additionally, it should be noted that e-liquids with an identical flavor label (e.g., melon from brand A and melon from brand B) might not consist of the same mixture of odor molecules and thus differ in the response pattern in the olfactory epithelium and beyond ${ }^{50}$. Hence, while our results justify orthonasal assessment of affective responses, additional research is needed to determine whether orthonasal smelling can also be used for assessments of sensory (perceptual) responses such as descriptive odor profiles of e-liquids. Finally, as smelling experiments were previously used to identify characterizing flavors in cigarettes and roll-your-own tobacco ${ }^{51}$, future research could focus on expanding the current results to flavors in other product types such as water pipe, cigars, and heated tobacco products.

\section{Concluding remarks}

We are the first to show that hedonic evaluation through orthonasal (smelling) and retronasal assessment (vaping) of e-liquid flavors yields comparable results, for both smokers and nonsmokers. This finding justifies the use of orthonasal smelling instead of vaping in several future studies, for example, investigating individuals who are nicotine-naïve (i.e., non-users) or under legal age for e-cigarette use (i.e., adolescents). Thus, smelling experiments, also being faster and less expensive than vaping, might be used to provide support for future regulations on e-liquid flavors. However, the more modest within-subject correlations and variation across individuals and specific e-liquid flavors suggest that the use of smelling instead of vaping may not be applicable to all research situations (e.g., for nicotine-containing e-liquids). Additional research is necessary to understand which variables tend to dissociate smelling vs. vaping ratings. 


\section{Appendix}

Appendix Table A7.1: Panel characteristics.

\begin{tabular}{|c|c|c|c|c|}
\hline & & $\begin{array}{l}\text { Whole group } \\
(\mathrm{n}=48)\end{array}$ & $\begin{array}{l}\text { Smokers } \\
(\mathrm{n}=24)\end{array}$ & $\begin{array}{l}\text { Non-smokers } \\
(\mathrm{n}=\mathbf{2 4}) *\end{array}$ \\
\hline \multirow[t]{2}{*}{ Gender } & Female & $50 \%$ & $50 \%$ & $50 \%$ \\
\hline & Male & $50 \%$ & $50 \%$ & $50 \%$ \\
\hline Mean age $( \pm$ SD) & & $24.8 \pm 8.4$ & $24.8 \pm 9.3$ & $24.9 \pm 7.7$ \\
\hline Age range & & $18-55$ & $18-54$ & $20-55$ \\
\hline Mean number of cigarettes/day ( & SD) & NA & $10.2 \pm 6.5$ & NA \\
\hline $\begin{array}{l}\text { Participants who inhaled during } \\
\text { vaping session via** }\end{array}$ & $\begin{array}{l}\text { Mouth only } \\
\text { Mouth and lungs } \\
\text { Don't remember }\end{array}$ & $\begin{array}{l}19 \\
28 \\
1\end{array}$ & $\begin{array}{l}1 \\
23 \\
0\end{array}$ & $\begin{array}{l}18 \\
5 \\
1\end{array}$ \\
\hline $\begin{array}{l}\text { Participants who exhaled during } \\
\text { vaping session via*** }\end{array}$ & $\begin{array}{l}\text { Mouth } \\
\text { Mouth and nose }\end{array}$ & $\begin{array}{l}36 \\
12 \\
\end{array}$ & $\begin{array}{l}16 \\
8\end{array}$ & $\begin{array}{l}20 \\
4 \\
\end{array}$ \\
\hline
\end{tabular}

* Among the group of non-smokers, there were 2 ex-smokers who reported to have quit smoking for 6 and 7 years, respectively.

** Inhalation and exhalation approach were asked retrospectively.

Appendix A7.2: Mean liking, intensity, familiarity, irritation ratings of e-liquid flavors.

Mean liking ratings ranged from $20.9 \pm 2.0$ (whiskey) to $68.4 \pm 2.1$ (mint) for smelling, and from 24.5 \pm 2.8 (whiskey) to $64.8 \pm 2.3$ (strawberry) for vaping (see Figure A7.2.1). Intensity ratings ranged from $20.4 \pm 2.7$ (PG/VG base) to $76.6 \pm 2.2$ (mint) for smelling, and from $23.9 \pm 2.5$ (PG/VG base) to $79.7 \pm$ 1.6 (mint) for vaping (see Figure A7.2.1). Familiarity ranged from $29.3 \pm 3.0$ (PG/VG base) to $84.7 \pm 1.4$ (mint) for smelling, and from $23.8 \pm 2.3$ (PG/VG base) to $85.7 \pm 1.2$ (mint) for vaping (see Figure A7.2.2). Irritation ranged from $15.9 \pm 2.3$ ( $\mathrm{PG} / \mathrm{VG}$ base) to $48.8 \pm 3.7$ (whiskey) for smelling, and from $14.5 \pm 1.6$ (PG/VG base) to $43.4 \pm 3.7$ (peppermint) for vaping (see Figure A7.2.2). 

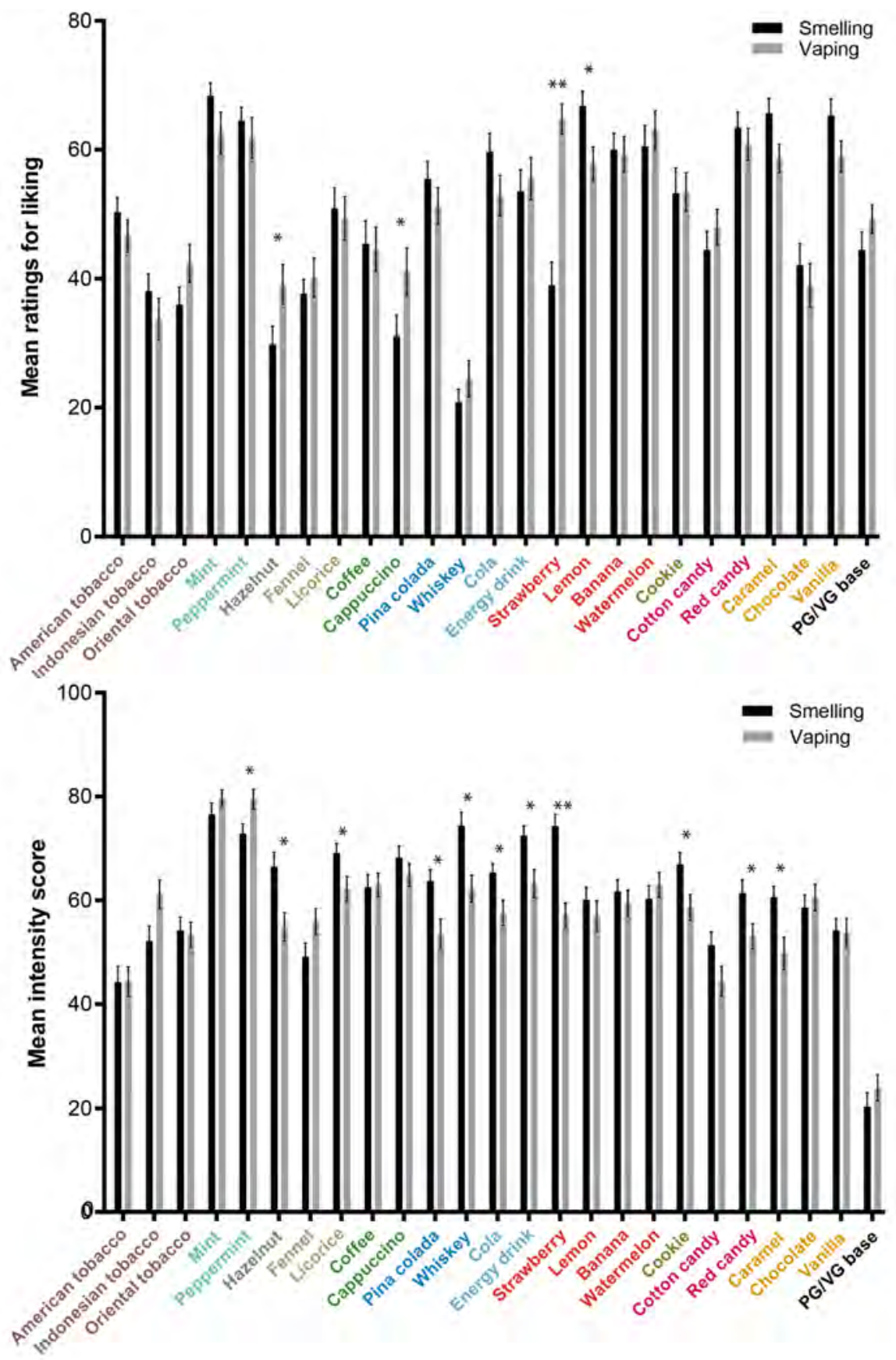

Appendix Figure A7.2.1: Mean ratings ( \pm SE) for liking (top) and perceived intensity (bottom) of each e-liquid flavor based on results from the whole group on a 100-mm Visual Analog Scale, for smelling (black) and vaping (grey). * Significant differences between smelling and vaping with $p<0.05$ (after false discovery rate correction); ${ }^{* *}$ Significant differences between smelling and vaping with $\mathrm{p}<0.001$ (after false discovery rate correction). 


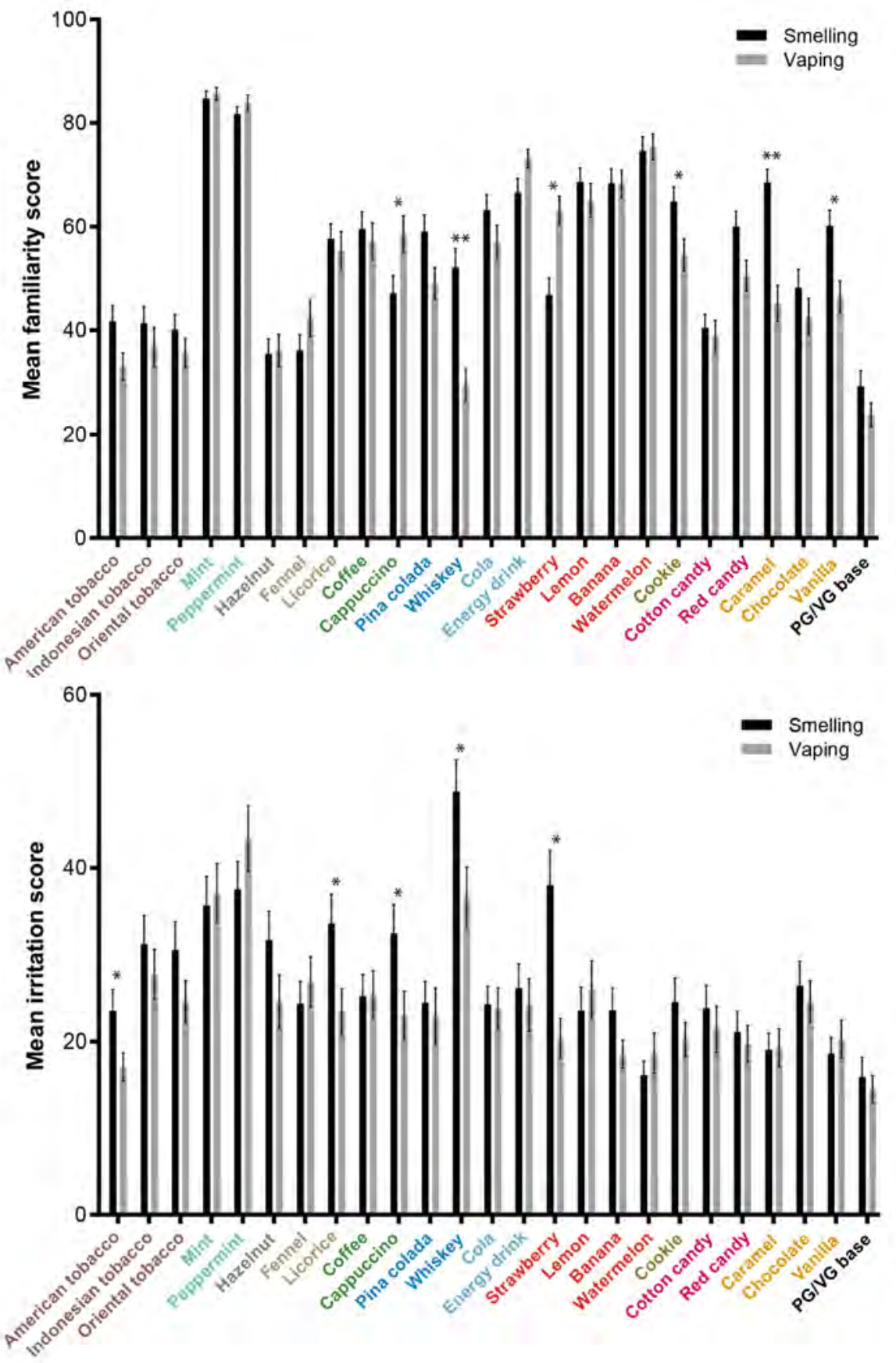

Appendix Figure A7.2.2: Mean ratings ( \pm SE) for familiarity (top) and perceived irritation (bottom) of each e-liquid flavor based on results from the whole group on a 100-mm Visual Analog Scale, for smelling (black) and vaping (grey). * Significant differences between smelling and vaping with $p<0.05$ (after false discovery rate correction); ${ }^{* *}$ Significant differences between smelling and vaping with $p<0.001$ (after false discovery rate correction). 
Appendix A7.3: Mean liking for smokers and non-smokers.

Mean liking ratings among smokers was highest for mint $(69.9 \pm 3.0)$ followed by peppermint $(67.8 \pm 2.6)$ in smelling, and for peppermint $(68.5 \pm 4.2)$ and mint $(65.4 \pm 4.9)$ in vaping (see Table A7.3.1). Among non-smokers, mean liking was highest for lemon $(67.9 \pm 3.3)$ and caramel $(67.2 \pm 2.6)$ in smelling, and for strawberry $(66.0 \pm 3.2)$ and watermelon $(64.2 \pm 3.7)$ in vaping.

Mean liking among smokers was lowest for whiskey $(19.9 \pm 3.0)$ and hazelnut $(27.3 \pm 4.5)$ in smelling, and whiskey $(26.7 \pm 4.7)$ and Indonesian tobacco (32.4 \pm 4.7$)$ in vaping. Among non-smokers, mean liking was lowest for whiskey $(21.9 \pm 2.5)$ and cappuccino $(30.8 \pm 4.4)$ in smelling, and for whiskey $(22.3 \pm 3.2)$ and Indonesian tobacco $(34.9 \pm 4.5)$ in vaping. However, differences in mean flavor liking between smokers and non-smokers were not significant. See Table A7.3.1 for a complete overview of the mean ratings for liking from both user groups.

Appendix A7.4: Correlations between smelling and vaping among smokers and non-smokers.

For smokers, the correlation coefficients based on the mean smelling and vaping ratings were 0.78 for liking, 0.76 for intensity, 0.83 for familiarity, and 0.65 for irritation. For non-smokers, the correlation coefficients based on the mean smelling and vaping ratings were 0.86 for liking, 0.85 for intensity, 0.84 for familiarity, and 0.75 for irritation. The correlations did not significantly differ between smokers and non-smokers.

Appendix A7.5: Correlations between smelling and vaping for liking.

The correlations for liking between smelling and vaping were positive for all individuals, except for two subjects $(R=-0.27$ and $R=-0.06)$. The positive correlation coefficients ranged from 0.06 to 0.87 . The correlation coefficients for liking of the individual e-liquid flavors between smelling and vaping ( $\mathrm{n}$ $=25)$ were all positive, except for whiskey $(R=-0.33)$. The positive correlations ranged between 0.08 (caramel) and 0.81 (watermelon). 


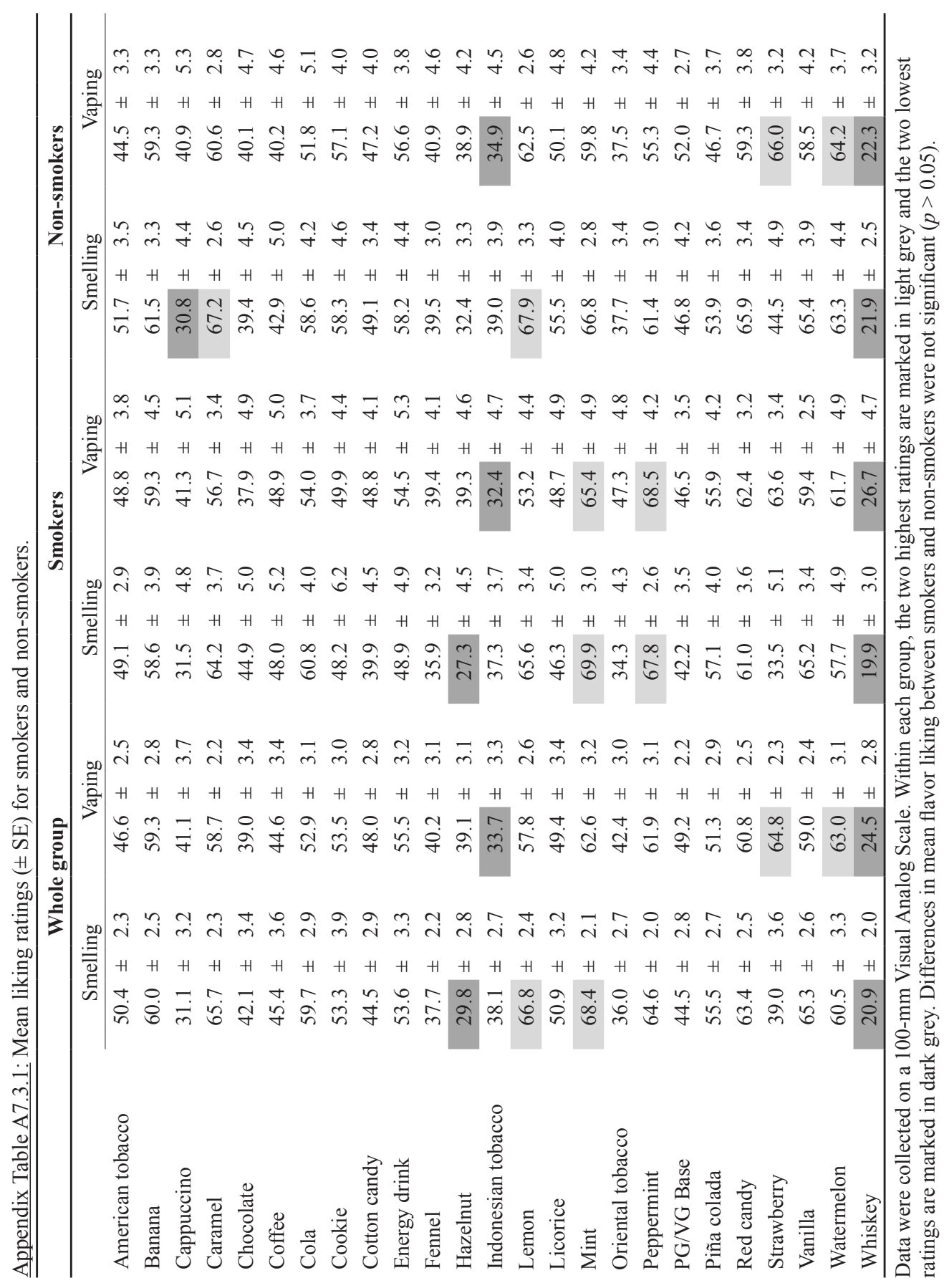




\section{References}

1. Filippidis FT, Laverty AA, Gerovasili V, Vardavas CI. Two-year trends and predictors of e-cigarette use in 27 European Union member states. Tob Control. 2017;26(1):98-104.

2. McMillen RC, Gottlieb MA, Shaefer RM, Winickoff JP, Klein JD. Trends in Electronic Cigarette Use Among U.S. Adults: Use is Increasing in Both Smokers and Nonsmokers. Nicotine Tob Res. 2015;17(10):1195-1202.

3. Romijnders K, van Osch L, de Vries H, Talhout R. Perceptions and Reasons Regarding E-Cigarette Use among Users and Non-Users: A Narrative Literature Review. Int J Environ Res Public Health. 2018;15(6).

4. Zare S, Nemati M, Zheng Y. A systematic review of consumer preference for e-cigarette attributes: Flavor, nicotine strength, and type. PLoS One. 2018;13(3):e0194145.

5. Zhu SH, Sun JY, Bonnevie E, et al. Four hundred and sixty brands of e-cigarettes and counting: implications for product regulation. Tob Control. 2014;23 Suppl 3:iii3-9.

6. Havermans A, Krüsemann EJZ, Pennings J, de Graaf K, Boesveldt S, Talhout R. Nearly 20000 e-liquids and 250 unique flavour descriptions: an overview of the Dutch market based on information from manufacturers. Tob Control. 2019:tobaccocontrol-2019-055303.

7. Diamantopoulou E, Barbouni A, Merakou K, Lagiou A, Farsalinos K. Patterns of e-cigarette use, biochemically verified smoking status and self-reported changes in health status of a random sample of vapeshops customers in Greece. Intern Emerg Med. 2019;14(6):843-851.

8. Pepper JK, Brewer NT. Electronic nicotine delivery system (electronic cigarette) awareness, use, reactions and beliefs: a systematic review. Tob Control. 2014;23(5):375-384.

9. Russell C, McKeganey N, Dickson T, Nides M. Changing patterns of first e-cigarette flavor used and current flavors used by 20,836 adult frequent e-cigarette users in the USA. Harm Reduct J. 2018;15(1):33.

10. Harrell MB, Weaver SR, Loukas A, et al. Flavored e-cigarette use: Characterizing youth, young adult, and adult users. Prev Med Reports. 2017;5:33-40.

11. Kong G, Morean ME, Cavallo DA, Camenga DR, Krishnan-Sarin S. Reasons for Electronic Cigarette Experimentation and Discontinuation Among Adolescents and Young Adults. Nicotine Tob Res. 2015;17(7):847-854.

12. Pepper JK, Ribis1 KM, Brewer NT. Adolescents' interest in trying flavoured e-cigarettes. Tob Control. 2016;25(Suppl 2):ii62-ii66.

13. Huang LL, Baker HM, Meernik C, Ranney LM, Richardson A, Goldstein AO. Impact of nonmenthol flavours in tobacco products on perceptions and use among youth, young adults and adults: a systematic review. Tob Control. 2017;26(6):709-719.

14. Villanti AC, Johnson AL, Ambrose BK, et al. Flavored Tobacco Product Use in Youth and Adults: Findings From the First Wave of the PATH Study (2013-2014). Am J Prev Med. 2017;53(2):139-151.

15. Prochaska JJ. The public health consequences of e-cigarettes: a review by the National Academies of Sciences. A call for more research, a need for regulatory action. Addiction. 2019;114(4):587-589.

16. Chen J, Bullen C, Dirks K. A Comparative Health Risk Assessment of Electronic Cigarettes and Conventional Cigarettes. Int J Environ Res Public Health. 2017;14(4).

17. Goniewicz ML, Gawron M, Smith DM, Peng M, Jacob P, 3rd, Benowitz NL. Exposure to Nicotine and Selected Toxicants in Cigarette Smokers Who Switched to Electronic Cigarettes: A Longitudinal Within-Subjects Observational Study. Nicotine Tob Res. 2017;19(2):160-167. 
18. Ratajczak A, Feleszko W, Smith DM, Goniewicz M. How close are we to definitively identifying the respiratory health effects of e-cigarettes? Expert Rev Respir Med. 2018;12(7):549-556.

19. Levy DT, Borland R, Lindblom EN, et al. Potential deaths averted in USA by replacing cigarettes with e-cigarettes. Tob Control. 2018;27(1):18-25.

20. McRobbie H. Modelling the Population Health Effects of E-Cigarettes Use: Current Data Can Help Guide Future Policy Decisions. Nicotine Tob Res. 2017;19(2):131-132.

21. Schneller LM, Bansal-Travers M, Goniewicz ML, McIntosh S, Ossip D, O’Connor RJ. Use of flavored electronic cigarette refill liquids among adults and youth in the US-Results from Wave 2 of the Population Assessment of Tobacco and Health Study (2014-2015). PLoS One. 2018;13(8):e0202744.

22. O'Connor RJ, Fix BV, McNeill A, et al. Characteristics of nicotine vaping products used by participants in the 2016 ITC Four Country Smoking and Vaping Survey. Addiction. 2019;114 Suppl 1:15-23.

23. Berg CJ. Preferred flavors and reasons for e-cigarette use and discontinued use among never, current, and former smokers. Int J Public Health. 2016;61(2):225-236.

24. Spence C. Multisensory flavor perception. Cell. 2015;161(1):24-35.

25. Green BG. Chemesthesis: Pungency as a component of flavor. Trends Food Sci Tech. 1996;7(12):415420.

26. McDonald ST, Bolliet DA, Hayes JE. Chemesthesis: Chemical Touch in Food and Eating. John Wiley \& Sons, Ltd.; 2016:1-7.

27. Goldenson NI, Kirkpatrick MG, Barrington-Trimis JL, et al. Effects of sweet flavorings and nicotine on the appeal and sensory properties of e-cigarettes among young adult vapers: Application of a novel methodology. Drug Alcohol Depend. 2016;168:176-180.

28. Rosbrook K, Green BG. Sensory Effects of Menthol and Nicotine in an E-Cigarette. Nicotine Tob Res. 2016;18(7):1588-1595.

29. Kim H, Lim J, Buehler SS, et al. Role of sweet and other flavours in liking and disliking of electronic cigarettes. Tob Control. 2016;25(Suppl 2):ii55-ii61.

30. Caggiula AR, Donny EC, Palmatier MI, Liu X, Chaudhri N, Sved AF. The role of nicotine in smoking: a dual-reinforcement model. Nebr Symp Motiv. 2009;55:91-109.

31. Peña y Lillo M, Latrille E, Casaubon G, Agosin E, Bordeu E, Martin N. Comparison between odour and aroma profiles of Chilean Pisco spirit. Food Qual Prefer. 2005;16:59-70.

32. Aubry V, Etievant P, Sauvageot F, Issanchou S. Sensory Analysis Of Burgundy Pinot Noir Wines: A Comparison Of Orthonasal And Retronasal Profiling. J Sens Stud. 1999;14:97-91 17.

33. Small DM, Gerber JC, Mak YE, Hummel T. Differential neural responses evoked by orthonasal versus retronasal odorant perception in humans. Neuron. 2005;47(4):593-605.

34. Rosbrook K, Green B. XXXIX Annual Meeting of the Association for Chemoreception Sciences Hyatt Regency Coconut Point, Bonita Springs, Florida. Chem Senses. 2018;43(4):e1-e136.

35. McBride RL. Stimulus range influences intensity and hedonic ratings of flavour. Appetite. $1985 ; 6(2): 125-131$.

36. Zandstra EH, De Graaf C, Mela DJ, Van Staveren WA. Short- and long-term effects of changes in pleasantness on food intake. Appetite. 2000;34(3):253-260.

37. Hummel T, Kobal G, Gudziol H, Mackay-Sim A. Normative data for the "Sniffin' Sticks" including tests of odor identification, odor discrimination, and olfactory thresholds: an upgrade based on a group of more than 3,000 subjects. Eur Arch Otorhinolaryngol. 2007;264(3):237-243. 
38. Krüsemann EJZ, Boesveldt S, de Graaf K, Talhout R. An E-Liquid Flavor Wheel: A Shared Vocabulary Based on Systematically Reviewing E-Liquid Flavor Classifications in Literature. Nicotine Tob Res. 2019;21(10):1310-1319.

39. Benjamini Y, Hochberg Y. Controlling the False Discovery Rate: A Practical and Powerful Approach to Multiple Testing. J Roy Stat Soc B Met. 1995;57(1):289-300.

40. De Graaf C. The validity of appetite ratings. Appetite. 1993;21(2):156-160.

41. Hummel T, Heilmann S, Landis BN, et al. Perceptual differences between chemical stimuli presented through the ortho- or retronasal route. Flavour Frag J. 2006;21(1):42-47.

42. Visschers RW, Jacobs MA, Frasnelli J, Hummel T, Burgering M, Boelrijk AE. Cross-modality of texture and aroma perception is independent of orthonasal or retronasal stimulation. J Agric Food Chem. 2006;54(15):5509-5515.

43. Moskowitz HR. Sensory intensity versus hedonic functions: classical psychophysical approaches. $J$ Food Quality. 1981;5:109-137.

44. Voirol E, Daget N. Comparative study of nasal and retronasal olfactory perception. Lebensm Wiss Technol. 1986;19:316-319.

45. Engelen L, de Wijk RA, Prinz JF, Janssen AM, Weenen H, Bosman F. The effect of oral and product temperature on the perception of flavor and texture attributes of semi-solids. Appetite. 2003;41(3):273281.

46. Bartoshuk LM, Rennert K, Rodin J, Stevens JC. Effects of temperature on the perceived sweetness of sucrose. Physiol Behav. 1982;28(5):905-910.

47. Vennemann MM, Hummel T, Berger K. The association between smoking and smell and taste impairment in the general population. $J$ Neurol. 2008;255(8):1121-1126.

48. Lawless H, Engen T. Associations to odors: interference, mnemonics, and verbal labeling. $J$ Exp Psychol-Hum L. 1977;3(1):52-59.

49. Herz RS. Odor-associative learning and emotion: effects on perception and behavior. Chem Senses. 2005;30 Suppl 1(suppl_1):i250-251.

50. Aszyk J, Kubica P, Wozniak MK, Namiesnik J, Wasik A, Kot-Wasik A. Evaluation of flavour profiles in e-cigarette refill solutions using gas chromatography-tandem mass spectrometry. J Chromatogr A. 2018;1547:86-98.

51. Krusemann EJZ, Lasschuijt MP, de Graaf C, et al. Sensory analysis of characterising flavours: evaluating tobacco product odours using an expert panel. Tob Control. 2019;28(2):152-160. 



\section{CHAPTER 8}

\section{Both non-smoking youth and smoking adults like sweet and minty e-liquid flavors more than tobacco flavor}

Erna J.Z. Krüsemann

Loes van Tiel

Jeroen L.A. Pennings

Wim Vaessen

Kees de Graaf

Reinskje Talhout

Sanne Boesveldt 


\begin{abstract}
Smokers may reduce their health risk by switching to electronic cigarette (e-cigarette) use. As e-cigarettes are not harmless, concerns exist about e-cigarette use by non-smokers and youth. E-cigarette refill solutions (e-liquids) are available in many different flavors that increase sensory appeal. Flavor preferences seem to differ between user groups, which may open doors for product regulation. We investigated which e-liquid flavors are attractive to specific user groups by comparing liking between adolescent non-smokers $(n=41$; mean age $16.9 \pm 0.8)$, young adult non-smokers $(n=42$; mean age $22.7 \pm 1.7)$, and adult smokers $(n=56$; mean age $39.7 \pm 11.1)$. Participants smelled tobacco $(n=6)$ and non-tobacco $(n=24)$ flavored e-liquids, and rated liking on a 9-point labeled hedonic scale, and familiarity, intensity, sweetness, bitterness, and irritation on a 100-unit Visual Analog Scale. Mean liking ranged from 2.3 (whiskey) to 6.7 (peppermint). Within all groups, the typically sweet and minty flavors (e.g., wine gum, watermelon, peppermint, menthol) were liked significantly more than the tobacco-flavored e-liquids. The set of tobaccoflavored e-liquids was significantly, but slightly, less disliked by adult smokers $(3.9 \pm 0.2)$ than adolescent $(3.1 \pm 0.3)$ and young adult $(3.4 \pm 0.3)$ non-smokers $(p<0.001)$. No between-group differences were observed for sweet and minty flavors. Liking correlated significantly positively with sweetness $(R=0.49)$ and familiarity $(R=0.48)$, and negatively with bitterness $(R=-0.58)$, irritation $(R=-0.47)$, and intensity $(R=-0.27)$. Thus, sweet and minty-flavored e-liquids are liked equally by young non-smokers and adult smokers, and more than tobacco flavors. Banning all flavors except tobacco will likely reduce e-cigarette appeal; potentially more for young nonsmokers than adult smokers.
\end{abstract}




\section{Introduction}

Sensory appeal, in particular taste and smell, is generally recognized as one of the most important motives for food choice ${ }^{1,2}$. Other industries, such as the tobacco industry, also use flavorings to increase sensory appeal of their products. For example, tobacco industry documents reveal that menthol is commonly added to cigarettes for its cooling, smoothing, and anesthetic effects, enhancing smoking behavior and nicotine dependence ${ }^{3,4}$.

E-cigarettes vaporize e-liquids that typically contain nicotine and are available in hundreds of different flavors ${ }^{5}$. E-liquid flavor categories include fruit, candy, tobacco, alcohol, dessert, and more ${ }^{6}$. Although e-cigarettes may attract smokers who aim to switch towards an alternative product in order to reduce their health risks ${ }^{7,8}$, the availability of appealing flavors also raises interest in e-cigarettes among adolescents and young adults who do not smoke ${ }^{9-12}$. However, as e-cigarette emissions contain toxic compounds and may facilitate nicotine dependence ${ }^{7,8}$, they are not harmless to health. Research also suggests that for adolescents and young adults, e-cigarettes may serve as a gateway product towards future initiation of cigarette smoking ${ }^{13}$. This makes regulation of e-cigarettes in order to reduce appeal and use among youth currently an important topic of debate ${ }^{14}$.

Although most e-cigarette users prefer and/or use e-liquids with a fruit or sweet flavor as well as traditional flavors such as tobacco ${ }^{15-26}$, flavor preferences seem to differ between (potential) user groups ${ }^{27,28}$. That is, young e-cigarette users typically report a preference for sweet flavors (e.g., candy, dessert and vanilla), while adults seem to be more attracted to non-sweet flavors (e.g., tobacco and menthol/mint) ${ }^{20,29,30}$. Also, smokers are more interested in trying tobacco and menthol flavored e-cigarettes than (young) non-smokers ${ }^{23,27,31,32}$, who are particularly interested in fruit and sweet flavors ${ }^{27,31,33,34}$. Most of these findings about e-liquid flavor preferences come from studies using surveys to collect data. Survey research is based on respondents' mental representation and memory of how they perceive a particular flavor, and is therefore an indirect approach to investigating flavor liking. Sensory research is a more direct approach as it allows respondents to actually taste or smell a sample when assessing its flavor. However, the amount of sensory research performed as an approach to investigating attractiveness of e-liquid flavors is limited. A few vaping studies showed that flavorings producing sweet or cooling sensations positively correlate with liking of e-cigarettes, while perceived bitterness and harshness/ irritation negatively correlate with liking ${ }^{22,35,36}$. Moreover, recent studies showed that appeal for e-cigarettes with fruit and menthol was higher than for tobacco-flavored e-cigarettes among current, former, as well as never smokers ${ }^{37}$, and that particularly green apple (fruit) flavor was liked by youth e-cigarette users ${ }^{38}$. Furthermore, olfaction (nose open) was found to contribute to liking and perceived sweetness of e-cigarette flavors more than taste (nose closed) ${ }^{39}$, and, in line with this, we previously showed that orthonasal smelling could be used as alternative to vaping when assessing sensory liking of e-liquid flavors ${ }^{40}$.

To build on this, liking of various e-liquid flavors could be compared between groups differing in age and smoking status. If, for example, flavors that attract current adult smokers but not youth and non-smokers were to be identified, this information could support regulators in their decisions on whether and how to decrease e-cigarette appeal for youth and non-smokers. Therefore, the current study aims to determine which flavors are attractive to specific user groups 
by investigating the hedonic assessment of e-liquids with various tobacco and non-tobacco flavors, among adolescent non-smokers, young adult non-smokers, and adult smokers by smelling. Familiarity and perceived sweetness, bitterness, intensity, and irritation of the e-liquid odors will be investigated as well, as these attributes are known to influence liking ${ }^{22}$. 


\section{Materials and methods}

\section{Participants}

Participants were recruited in and around the cities Ede and Utrecht (the Netherlands) by Essensor $\mathrm{BV}$, a company specialized in sensory market research that uses large recruitment databases and targeted search methods (i.e., via email, social media, word-of-mouth, WhatsApp, and by phone) to recruit representative participants. Inclusion criteria, assessed using a self-report questionnaire, were: being an adolescent non-smoker (aged 16-18), young adult non-smoker (aged 20-25), or adult smoker (aged 20-55); having ever heard of the e-cigarette prior to this study; being healthy; and having a good proficiency of the Dutch language. Non-smokers were defined as reporting to have smoked less than 100 tobacco cigarettes in their lifetime, and reporting to currently not smoke cigars, pipe, or marihuana. Smokers were defined as reporting to have smoked more than 100 tobacco cigarettes in their lifetime (excluding cigars, pipe, or marihuana) and currently smoking tobacco cigarettes on a daily basis or more than once per week. Participants were not required to have ever used e-cigarettes. Exclusion criteria were: being pregnant or lactating; having self-reported olfactory deficiencies; being employed or performing thesis research at the Division of Human Nutrition and Health of Wageningen University; and participating in other medical-scientific research.

The study was originally powered for $\mathrm{n}=56$ per group. Sample size was determined using data from our previous sensory study where the absolute difference in mean scores for liking of e-liquid flavors ( $n=25$; assessed by means of smelling) between user groups (smokers and nonsmokers) ranged from 0.2 to 11 on a 100 -unit Visual Analog Scale (VAS) ${ }^{40}$. We calculated that 56 participants are needed per group in order to identify significant differences between the group means of at least $15 / 100$ points, which corresponds to 1.35 points on a 9-point hedonic scale, with more than $90 \%$ power and a significance level of at $p<0.05$ after applying a correction for multiple testing.

Participants who completed the study received a financial compensation. All participants provided written informed consent prior to the first test session. The study was approved by the Medical Ethical Committee of Wageningen University (METC 19/27; NL72171.081.19), and registered in the Dutch Trial Register (ID: NL8333).

\section{E-liquid products}

Thirty commercial e-liquids, from 14 different brands, were purchased from 10 different online shops. The e-liquids' base consisted of various propylene glycol (PG) to vegetable glycerin (VG) ratios, and, for ethical reasons since adolescents and non-smokers were included, contained 0 $\mathrm{mg} / \mathrm{mL}$ nicotine. E-liquid flavor selection was based on the different categories of the e-liquid flavor wheel ${ }^{6}$. The e-liquids' odors were evaluated by the research team during a preliminary experiment to ensure inclusion of odor qualities that were distinct and that matched the e-liquid flavor name. We selected 6 e-liquids from the tobacco category to ensure a strong representation of this traditional category, 1 unflavored e-liquid as a blank sample, 1 e-liquid from the other flavors category, and two e-liquids from the remaining non-tobacco categories of the e-liquid flavor wheel to optimize flavor variety. See Table 8.1 for an overview of the products included. 
Table 8.1: E-liquid products $(\mathrm{n}=30)$ used in this study, including their flavor category and dilution factor.

\begin{tabular}{|c|c|c|c|}
\hline & Flavor category & Flavor & \# drops diluted in $1 \mathrm{~mL}$ demi-water \\
\hline 1 & Tobacco & American blend & 15 \\
\hline 2 & Tobacco & Cigar & 5 \\
\hline 3 & Tobacco & Tobacco_a & 5 \\
\hline 4 & Tobacco & Tobacco_b & 10 \\
\hline 5 & Tobacco & Tobacco_c & 3 \\
\hline 6 & Tobacco & Oriental & 12 \\
\hline 7 & Menthol/mint & Peppermint & 5 \\
\hline 8 & Menthol/mint & Menthol & 3 \\
\hline 9 & Nuts & Hazelnut & 3 \\
\hline 10 & Nuts & Peanut & 1 \\
\hline 11 & Spices & Anise & 5 \\
\hline 12 & Spices & Clove & 5 \\
\hline 13 & Coffee/tea & Jasmine tea & 10 \\
\hline 14 & Coffee/tea & Espresso & 10 \\
\hline 15 & Alcohol & Whiskey & 5 \\
\hline 16 & Alcohol & Mojito & 5 \\
\hline 17 & Other beverages & Energy drink & 10 \\
\hline 18 & Other beverages & Cola & 5 \\
\hline 19 & Fruit (berries) & Raspberry & 10 \\
\hline 20 & Fruit (citrus) & Citrus fruits & 5 \\
\hline 21 & Fruit (tropical) & Pineapple & 3 \\
\hline 22 & Fruit (other) & Watermelon & 5 \\
\hline 23 & Dessert & Syrup waffle & 2 \\
\hline 24 & Dessert & Cheesecake & 2 \\
\hline 25 & Candy & Bubblegum & 10 \\
\hline 26 & Candy & Wine gum & 1 (in $10 \mathrm{~mL})$ \\
\hline 27 & Other sweets & Caramel & 10 \\
\hline 28 & Other sweets & Vanilla & 10 \\
\hline 29 & Other flavors & Lavender & 3 \\
\hline 30 & Unflavored & PG/VG base only & 1 \\
\hline
\end{tabular}

NB: $\mathrm{a}, \mathrm{b}$, and $\mathrm{c}$ for product 3,4 , and 5 represent three different e-liquid products that were all marketed as having an (unspecified) tobacco flavor.

PG, propylene glycol; VG, vegetable glycerin. 


\section{Sample preparation}

Several e-liquid drops were dissolved in $1 \mathrm{~mL}$ demineralized water and put in a $60 \mathrm{~mL}$ brown glass vial. The number of drops per e-liquid is shown in Table 8.1 (final column), and was based on a pilot experiment in order to standardize odor intensity. In this experiment, 10 participants assessed odor intensity of various dilutions on a 100-unit VAS (left anchor: "not intense at all"; right anchor: "very intense"), until the mean intensity was between 50 and 75 (i.e., not too weak nor too strong). Vials were filled with e-liquid on the same day or one day before a test session, and labelled with a random three-digit code. A new set of samples was prepared for each participant to standardize sample intensity. E-liquids were stored at room temperature in their original package.

\section{Experimental procedure}

The test sessions took place at two different locations (Ede and Utrecht, Essensor BV, the Netherlands). Participants took place in sensory booths equipped with a computer; water and tissues were provided. The room was accommodated with a controlled high capacity ventilation system. Participants were asked to refrain from using scented crèmes, deodorant and perfumes on test days, and to eat or drink nothing other than water (including chewing gum, using tooth paste, and smoking) at least 1 hour prior to their test visit. For each participant, two test sessions of 1 hour each were scheduled on two consecutive days during which they assessed the 30 e-liquids in total in balanced order on liking, familiarity, sweetness, bitterness, intensity, and irritation.

EyeQuestion software V.4.11.68 (Logic8 BV) was used for data collection. Participants were allowed to smell the samples as often as needed to answer all questions. Each product was firstly assessed on liking ("imagine you are using an e-cigarette, how much do you like the odor of this e-liquid?") using a 9-point labeled hedonic scale. This was followed by familiarity ("how familiar are you with this odor?"), perceived sweetness, bitterness, and intensity ("how sweet/bitter/intense do you perceive this e-liquid's odor?"), and irritation ("to what extent do you perceive an irritating feeling in your nose due to this e-liquid's odor?") using 100-unit Visual Analog Scales (left anchor "not at all"; right anchor "very much"). To prevent olfactory adaptation, a one-minute break was set between each sample during which participants were instructed to smell their own clothing and rinse their mouth with water.

After assessment of the final sample, participants answered closed questions about their educational level, intention to start vaping, history of e-cigarette use (including flavor and nicotine level of most recent e-cigarette, and reason for use). This was followed by a question about their interest in trying specific e-cigarette flavors (check all that apply). Participants reported how often they eat/drink/use (8-point category scale from never to daily) and how much they like (9-point labeled hedonic scale) products with the flavors included in this study. The group of smokers answered additional questions about smoking history and quit intention, and filled out the Fagerström Test For Nicotine Dependency (FTND) ${ }^{41}$.

\section{Data analysis}

R statistical software V.4.0.2 (including "stringr" and "psych" packages) was used for data analysis. Of the 141 participants included in the study, 139 completed the experiment and those were used for analysis. Results were compared between user groups and between flavors. 


\section{Panel characteristics}

Means and percentages of the answers to each survey item were calculated, for the whole group and for the three separate user groups. Some answer options were combined and recoded into a different answer category; these can be can be found in Appendix Table A8.1.

\section{Between-group comparisons}

For each attribute (liking, familiarity, sweetness, bitterness, intensity, and irritation), a one-way ANOVA was performed to determine differences in the assessment of individual e-liquids and across all e-liquids $(n=30)$ between the following user groups: adolescent non-smokers and young adult non-smokers (both separately and combined into one group of non-smokers), and adult smokers. Liking was also compared between these user groups for 4 sets of products with similar flavors (excluding the unflavored e-liquid). Categorization of these 4 product groups was based on similarities in the type of flavor (flavor category) and in sweetness ratings (see Appendix Table A8.4 for sweetness data): tobacco flavors $(n=6$; American blend, cigar, oriental, tobacco_a,b,c), minty flavors $(\mathrm{n}=2$; menthol, peppermint), other non-sweet flavors $(\mathrm{n}=5$; whiskey, espresso, clove, peanut, hazelnut), and sweet flavors $(\mathrm{n}=16$; the remaining products, which were those with the highest sweetness ratings). Product, user group, and gender were included as covariates in the ANOVA model. When $p$-values were significant, post-hoc $t$-tests were performed to test differences between groups. A Benjamini-Hochberg false discovery rate correction was applied to the $p$-values in order to corrected for multiple testing ${ }^{42}$; adjusted $p$-values of $\leq 0.05$ were considered significant.

\section{Between-product comparisons}

Mean ratings and standard error over the means were calculated for each product, in total and per user group. For each outcome, ratings were compared for each combination of two e-liquids, using paired $t$-tests to account for participants' repeated (paired) measurements. This was done for each user group separately. A Benjamini-Hochberg false discovery rate correction was applied 42; adjusted $p$-values of $\leq 0.05$ were considered significant.

\section{Correlations}

Pearson correlations between liking, familiarity, sweetness, bitterness, intensity, and irritation were calculated using ratings across all products. This was done across all users and for the individual user groups. In addition, per attribute, for the total sample and for each user group separately, Pearson correlations were calculated between sensory e-liquid ratings and selfreported ratings for (1) general use and (2) liking of other (often food) products with the same flavors as those of the e-liquids included in this study. Corresponding $p$-values were corrected for multiple testing using the Benjamini-Hochberg false discovery rate correction ${ }^{42}$. 


\section{Results}

\section{Panel characteristics}

The final sample consisted of 41 adolescent non-smokers (61\% female; mean age $16.9 \pm 0.8)$, 42 young adult non-smokers ( $86 \%$ female; mean age $22.7 \pm 1.7)$, and 56 adult smokers $(57 \%$ female; mean age $39.7 \pm 11.1)$. Although more than half of the participants $(58 \%)$ reported to have ever or regularly used an e-cigarette, most people within all groups had no intention to start vaping. Of the ever or regular e-cigarette users, most vaped fruit or menthol/mint flavor in the e-cigarette they most recently used. For adult smokers, this was mostly menthol/mint flavor, followed by tobacco flavor. All panel characteristics are shown in Table 8.2.

\section{Between-group comparisons}

The group of tobacco-flavored e-liquids was significantly less disliked by adult smokers (mean \pm SE: $3.9 \pm 0.2)$ than adolescent non-smokers $(3.1 \pm 0.3 ; p<0.001)$ and young adult non-smokers $(3.4 \pm 0.3 ; p<0.001)$, both separately and combined $(p<0.001)$. The tobacco-flavored e-liquids were also significantly less disliked by young adult than adolescent non-smokers $(p=0.009)$. Similarly, the group of other non-sweet flavors was significantly less disliked by adult smokers (mean \pm SE: $3.7 \pm 0.3$ ) compared to adolescent non-smokers $(3.2 \pm 0.3 ; p<0.001)$, and compared to the combined group of young adult $(3.4 \pm 0.3)$ and adolescent non-smokers $(p=0.002)$. Liking of both the sets of menthol/mint-flavored e-liquids and sweet e-liquids did not significantly differ between the user groups.

As regards to individual e-liquids, liking ratings for 28 of the 30 products did not significantly differ between adolescent non-smokers, young adult non-smokers, and adult smokers (Figure 8.1). One e-liquid from the tobacco category (American blend) was less disliked by adult smokers (mean \pm SE: $4.9 \pm 0.2)$ compared to young adult $(3.8 \pm 0.3)$ and adolescent non-smokers $(3.5 \pm 0.3)$, both separately and combined $(p<0.001)$. Another tobacco-flavored e-liquid (Oriental flavor), was less disliked by adult smokers (mean \pm SE: $4.3 \pm 0.3$ ) and young adult non-smokers $(4.3 \pm 0.3)$ than adolescent non-smokers $(3.0 \pm 0.2)(p<0.001$ for both). Neither familiarity, intensity, sweetness, bitterness, nor irritation differed significantly between the groups for any of the individual products $(p>0.05)$. Mean liking ratings for all (groups of) products are shown in Appendix Table A8.2. 
Table 8.2: Characteristics of the panelists included in this study.

\begin{tabular}{|c|c|c|c|c|c|}
\hline & & $\begin{array}{l}\text { Total sample } \\
(\mathrm{n}=139)\end{array}$ & $\begin{array}{l}\text { Adolescent } \\
\text { non-smokers } \\
(\mathrm{n}=41)\end{array}$ & $\begin{array}{l}\text { Young adult } \\
\text { non-smokers } \\
(\mathrm{n}=42)\end{array}$ & $\begin{array}{l}\begin{array}{l}\text { Adult } \\
\text { smokers } \\
(\mathrm{n}=56)\end{array} \\
\end{array}$ \\
\hline Mean age \pm SD & & $27.8 \pm 12.3$ & $16.9 \pm 0.8$ & $22.7 \pm 1.7$ & $39.7 \pm 11.1$ \\
\hline \multirow[t]{2}{*}{ Gender (\%) } & Women & 67 & 61 & 86 & 57 \\
\hline & Men & 33 & 39 & 14 & 43 \\
\hline \multirow{3}{*}{$\begin{array}{l}\text { Education level } \\
(\%)\end{array}$} & Low & 24 & 51 & 5 & 20 \\
\hline & Middle & 49 & 49 & 40 & 55 \\
\hline & High & 27 & 0 & 55 & 25 \\
\hline \multirow{3}{*}{$\begin{array}{l}\text { History of } \\
\text { e-cigarette use (\%) }\end{array}$} & Never & 42 & 54 & 64 & 16 \\
\hline & Ever & 43 & 41 & 36 & 50 \\
\hline & Regularly & 15 & 5 & 0 & 34 \\
\hline \multirow{13}{*}{$\begin{array}{l}\text { Most recent flavor } \\
\text { (\% of ever/regular } \\
\text { users in that group) }\end{array}$} & Fruit & 28 & 63 & 27 & 15 \\
\hline & Menthol/mint & 27 & 32 & 33 & 23 \\
\hline & $\begin{array}{l}\text { Other sweets (vanilla } \\
\text { or chocolate) }\end{array}$ & 12 & 0 & 20 & 15 \\
\hline & Tobacco & 11 & 0 & 0 & 19 \\
\hline & Unflavored & 7 & 5 & 0 & 11 \\
\hline & Candy & 4 & 0 & 7 & 4 \\
\hline & Nuts & 2 & 0 & 0 & 4 \\
\hline & Other beverages & 2 & 0 & 7 & 2 \\
\hline & Spices & 2 & 0 & 0 & 4 \\
\hline & Coffee/tea & 1 & 0 & 0 & 2 \\
\hline & Don’t know & 1 & 0 & 7 & 0 \\
\hline & Dessert & 0 & 0 & 0 & 0 \\
\hline & Alcohol & 0 & 0 & 0 & 0 \\
\hline \multirow{2}{*}{$\begin{array}{l}\text { Most recent } \\
\text { nicotine level }\end{array}$} & No nicotine & 9 & 21 & 20 & 0 \\
\hline & $1-8 \mathrm{mg} / \mathrm{mL}$ & 22 & 26 & 13 & 23 \\
\hline \multirow{3}{*}{$\begin{array}{l}\text { ( } \% \text { of ever/regular } \\
\text { users in that group) }\end{array}$} & $9-20 \mathrm{mg} / \mathrm{mL}$ & 12 & 0 & 0 & 21 \\
\hline & $>20 \mathrm{mg} / \mathrm{mL}$ & 0 & 0 & 0 & 0 \\
\hline & Don't know & 57 & 53 & 67 & 55 \\
\hline \multirow{5}{*}{$\begin{array}{l}\text { Reason for } \\
\text { e-cigarette use } \\
\text { (\% of ever/regular } \\
\text { users in that group) }\end{array}$} & Curiosity & 67 & 79 & 80 & 57 \\
\hline & Health reasons & 4 & 0 & 0 & 6 \\
\hline & To quit smoking & 16 & 0 & 0 & 28 \\
\hline & Friends use it too & 12 & 21 & 20 & 6 \\
\hline & $\begin{array}{l}\text { Other ("it smelled } \\
\text { nice") }\end{array}$ & 1 & 0 & 0 & 2 \\
\hline \multirow{6}{*}{$\begin{array}{l}\text { Interest in } \\
\text { e-cigarette flavor } \\
\text { (n; check all that } \\
\text { apply) }\end{array}$} & Fruit & 97 & 35 & 28 & 34 \\
\hline & Menthol/mint & 92 & 31 & 26 & 35 \\
\hline & Candy & 62 & 23 & 17 & 22 \\
\hline & $\begin{array}{l}\text { Other sweets (vanilla } \\
\text { or chocolate) }\end{array}$ & 51 & 11 & 17 & 23 \\
\hline & Spices & 36 & 7 & 12 & 17 \\
\hline & Tobacco & 32 & 2 & 5 & 25 \\
\hline
\end{tabular}




\begin{tabular}{|c|c|c|c|c|c|}
\hline & Other beverages & 32 & 15 & 9 & 8 \\
\hline & Dessert & 29 & 8 & 10 & 11 \\
\hline & Alcohol & 27 & 9 & 10 & 8 \\
\hline & Coffee/tea & 24 & 5 & 8 & 11 \\
\hline & Nuts & 18 & 2 & 8 & 8 \\
\hline \multirow{4}{*}{$\begin{array}{l}\text { Intention to start } \\
\text { vaping (\%) }\end{array}$} & No intention & 59 & 76 & 79 & 32 \\
\hline & Low intention & 14 & 2 & 5 & 29 \\
\hline & High intention & 4 & 0 & 0 & 11 \\
\hline & Don’t know & 23 & 22 & 17 & 29 \\
\hline \multirow{4}{*}{$\begin{array}{l}\text { Intention to quit } \\
\text { smoking* }(\%)\end{array}$} & No intention & & & & 18 \\
\hline & Low intention & & & & 42 \\
\hline & High intention & & & & 33 \\
\hline & Don’t know & & & & 7 \\
\hline \multirow{4}{*}{$\begin{array}{l}\text { Smoking duration* } \\
(\%)\end{array}$} & $<1$ year & & & & 0 \\
\hline & $1-5$ years & & & & 9 \\
\hline & $5-10$ years & & & & 9 \\
\hline & $>10$ years & & & & 82 \\
\hline \multirow{5}{*}{$\begin{array}{l}\text { Number of } \\
\text { cigarettes per day* } \\
(\%)\end{array}$} & 1-10 (less than ha & ackage) & & & 42 \\
\hline & 11-19 (more than & a package) & & & 38 \\
\hline & 20 (1 package $)$ & & & & 5 \\
\hline & 21-25 (more than & kage) & & & 11 \\
\hline & $\begin{array}{l}\text { I have not smokec } \\
\text { regularly }\end{array}$ & & & & 4 \\
\hline \multirow{3}{*}{$\begin{array}{l}\text { Cigarette flavor } \\
\text { most often used* } \\
(\%)\end{array}$} & Tobacco & & & & 95 \\
\hline & Menthol & & & & 5 \\
\hline & Other & & & & 0 \\
\hline \multirow{3}{*}{$\begin{array}{l}\text { Ever use of } \\
\text { cigarettes with } \\
\text { menthol or other } \\
\text { flavor* (n; check } \\
\text { all that apply) }\end{array}$} & No & & & & 7 \\
\hline & Menthol & & & & 43 \\
\hline & $\begin{array}{l}\text { Flavor other than } \\
\text { menthol }\end{array}$ & & & & 12 \\
\hline \multirow{4}{*}{$\begin{array}{l}\text { Nicotine } \\
\text { dependence* }{ }^{41}(\%)\end{array}$} & Low dependence & & & & 40 \\
\hline & $\begin{array}{l}\text { Low to moderate } \\
\text { dependence }\end{array}$ & & & & 33 \\
\hline & Moderate depend & & & & 27 \\
\hline & High dependence & & & & 0 \\
\hline
\end{tabular}

General note: percentages may not add up to 100 due to rounding; interest in e-cigarette flavor was asked after product assessment and may thus have been influenced thereby.

* Only applicable to the group of adult smokers $(\mathrm{n}=55)$; missing data for 1 participant. 

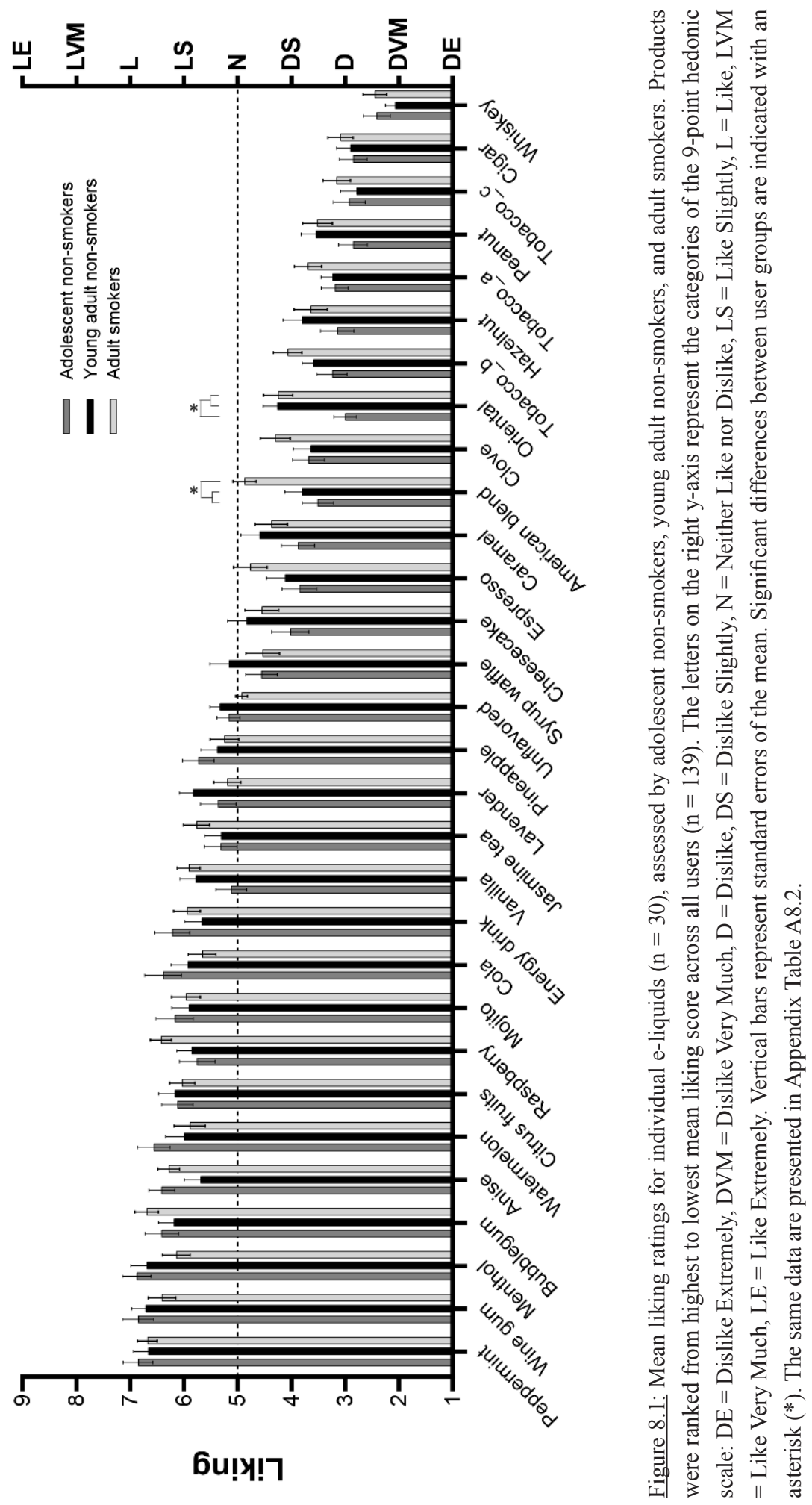


\section{Between-product comparisons}

Across all users, mean liking ratings ranged from 2.3 (whiskey) to 6.7 (peppermint) on a 9-point hedonic scale (Figure 8.1 and Appendix Table A8.2). All user groups generally liked menthol/mint- and sweet-flavored e-liquids more than tobacco and other non-sweet e-liquids. Specifically, for all three user groups, liking ratings for e-liquids with peppermint, wine gum, menthol, bubblegum, anise, watermelon, citrus fruits, raspberry, mojito, cola, energy drink, vanilla, and jasmine tea flavors were significantly higher than liking ratings of the 6 tobaccoflavored e-liquids, clove, hazelnut, peanut, and whisky $(p \leq 0.05)$. Mean ratings for the menthol/ mint and most sweet e-liquid flavors typically ranged between 5.4 (pineapple; across-group average) and 6.7 (peppermint), which corresponds to "Neither Like nor Dislike" (5 points) and "Like" (7 points). Mean ratings for e-liquids with a tobacco or non-sweet flavor typically ranged between 2.3 (whiskey) and 4.3 (espresso), which corresponds to "Dislike Very Much" (2 points) and "Dislike Slightly" (4 points).

Across all users, mean familiarity ratings ranged from 20.4 (unflavored) to 83.8 (peppermint) on a VAS from 0 to 100 (Appendix Table A8.3). Participants were particularly familiar with the minty e-liquid odors (mean ratings above 72.2 for all user groups). Specifically, e-liquids flavored as peppermint, menthol, and anise were rated as significantly more familiar (78.6 on average) than all 15 e-liquids (half of total sample) with the lowest mean familiarity ratings (39.3 on average) within all user groups $(p \leq 0.05)$.

Mean ratings for perceived sweetness ranged from 22.5 (unflavored) to 79.6 (energy drink) across all users (Appendix Table A8.4). Sweetness ratings differed significantly between the e-liquids. Within all user groups, e-liquids flavored as energy drink, wine gum, bubblegum, watermelon, raspberry, citrus fruits, pineapple, and anise were perceived as significantly more sweet ( 71.5 on average) than all 15 e-liquids (half of total sample) with the lowest sweetness ratings ( 34.3 on average) $(p \leq 0.05)$.

Mean ratings for perceived bitterness ranged from 12.8 (wine gum) to 64.7 (whiskey) across all users. Bitterness differed between the e-liquids, in such a way that whiskey, tobacco $(n=6)$, espresso, peanut, clove, and hazelnut flavored e-liquids were rated as significantly more bitter (52.9 on average) than all 15 e-liquids (half of total sample) with the lowest bitterness ratings (20.9 on average) within all user groups ( $p \leq 0.05)$.

Excluding the unflavored e-liquid (12.7 points), mean ratings for perceived odor intensity ranged from 44.5 (vanilla) to 73.0 (whiskey) across all users. Between-product differences that were found within all user groups were the following: the whiskey-flavored e-liquid was rated as significantly more intense than anise, bubblegum, wine gum, watermelon, pineapple, raspberry, American blend, vanilla, and unflavored (48.5 on average) $(p \leq 0.05)$; and the unflavored e-liquid was rated as significantly less intense than all other products $(p \leq 0.05)$.

Finally, mean ratings for irritation ranged from 8.6 (unflavored) to 59.7 (whiskey). The between-product differences that were found within all user groups concerned the whiskeyflavored e-liquid, which was rated as significantly more irritating than the 15 e-liquids (half of total sample) with the lowest irritation ratings ( 25.7 on average) $(p \leq 0.05)$. 


\section{Correlations between attributes}

Liking significantly positively correlated with sweetness $(R=0.49)$ and familiarity $(R=0.48)$, and negatively with bitterness $(R=-0.58)$, intensity $(\mathrm{R}=-0.27)$, and irritation $(R=-0.47)$ (see Table 8.3 for all correlation coefficients). The correlation coefficient between liking and sweetness was significantly stronger for adolescent non-smokers $(R=0.58)$ compared to young adult non-smokers $(R=0.44 ; p<0.001)$ and adult smokers $(R=0.46 ; p<0.001)$. Similarly, the correlation coefficient between liking and bitterness was significantly stronger for adolescent non-smokers $(R=-0.64)$ than young adult non-smokers $(R=-0.57 ; p=0.006)$ and adult smokers $(R=-0.55 ; p<0.001)$.

Table 8.3: Pearson correlation coefficients $(R)$ between the attributes, across all users $(\mathrm{n}=139)$ and products $(\mathrm{n}=30)$. As expected, all correlations were significant, except sweetness vs. intensity.

\begin{tabular}{lrrrrrr}
\hline & Liking & Familiarity & Sweetness & Bitterness & Intensity & Irritation \\
\hline Liking & n.a. & & & & & \\
Familiarity & $0.48^{*}$ & n.a. & & & & \\
Sweetness & $0.49^{*}$ & $0.40^{*}$ & n.a. & & & \\
Bitterness & $-0.58^{*}$ & $-0.27^{*}$ & $-0.46^{*}$ & n.a. & & \\
Intensity & $-0.28^{*}$ & $0.16^{*}$ & 0.02 & $0.30^{*}$ & n.a. & \\
Irritation & $-0.47^{*}$ & $-0.15^{*}$ & $-0.25^{*}$ & $0.52^{*}$ & $0.44^{*}$ & n.a. \\
\hline
\end{tabular}

* Significant correlations with $p \leq 0.05$ after correcting for multiple testing.

\section{Correlations between sensory assessment and general product use/liking}

Across all users, sensory ratings of e-liquid flavors (all attributes) did not correlate significantly with participants' self-reported frequency of eating, drinking, or using a product with the same flavor for any of the attributes $(p>0.05)$ (Table 8.4). Within both groups of young non-smokers significant positive, but weak, correlations were found: the extent to which the non-smokers eat, drink, or use a product with a particular flavor correlated significantly with liking $(R=0.30$ for adolescents and $R=0.27$ for young adults) and familiarity ( $R=0.24$ and 0.22$)$ of an e-liquid with that flavor label, which means that the more often they used a product in daily life, the more they liked and were familiar with the associated e-liquid odor. In addition, the more often adolescent non-smokers used a product with a certain flavor in daily life, the more sweet $(R=0.26)$ and less bitter $(R=-0.23)$ they perceived an e-liquid with that flavor label.

Across all users, sensory assessment of e-liquid flavors correlated significantly weakly positively for liking $(R=0.32)$, familiarity $(R=0.22)$, and sweetness $(R=0.24)$, and significantly weakly negatively for bitterness $(R=-0.25)$, with how much the participants reported to like products with that particular flavor in daily life (according to survey questions) (Table 8.4). This means that the smell of e-liquids with the same flavor as a product they like in daily life were 
rated higher on sensory liking, familiarity, and perceived sweetness, and lower on bitterness than those e-liquids with the same flavor as a product they dislike in daily life. Similar correlations were found for the groups of adolescent and young adult non-smokers separately, but not for adult smokers.

Table 8.4: Pearson correlation coefficients $(R)$ between frequency of eating, drinking, or using a product with a particular flavor in daily life and sensory assessment of e-liquid flavors with the same flavor label.

\begin{tabular}{llrrrr}
\hline Correlations (R) & & $\begin{array}{r}\text { Total } \\
\text { sample }\end{array}$ & $\begin{array}{r}\text { Adolescent } \\
\text { non- } \\
\text { smokers }\end{array}$ & $\begin{array}{r}\text { Young } \\
\text { adult non- } \\
\text { smokers }\end{array}$ & $\begin{array}{r}\text { Adult } \\
\text { smokers }\end{array}$ \\
\hline How often do & Liking of e-liquid flavor X & 0.15 & $0.30^{*}$ & $0.27^{*}$ & -0.03 \\
you eat/drink/ & Familiarity of e-liquid flavor X & 0.06 & $0.24^{*}$ & $0.22^{*}$ & -0.11 \\
use a product & Sweetness of e-liquid flavor X & 0.05 & $0.26^{*}$ & 0.16 & -0.13 \\
with flavor X? & Bitterness of e-liquid flavor X & -0.06 & $-0.23^{*}$ & -0.18 & 0.08 \\
& Intensity of e-liquid flavor X & -0.02 & 0.01 & 0.04 & -0.09 \\
& Irritation of e-liquid flavor X & -0.03 & -0.08 & -0.02 & -0.04 \\
\hline How much & Liking of e-liquid flavor X & $0.32^{*}$ & $0.38^{*}$ & $0.40^{*}$ & 0.18 \\
do you like a & Familiarity of e-liquid flavor X & $0.22^{*}$ & $0.33^{*}$ & $0.33^{*}$ & 0.05 \\
product with & Sweetness of e-liquid flavor X & $0.24^{*}$ & $0.38^{*}$ & $0.31^{*}$ & 0.08 \\
flavor X? & Bitterness of e-liquid flavor X & $-0.25^{*}$ & $-0.33^{*}$ & $-0.34^{*}$ & -0.12 \\
& Intensity of e-liquid flavor X & -0.06 & -0.03 & -0.05 & -0.10 \\
& Irritation of e-liquid flavor X & -0.14 & -0.15 & -0.16 & -0.15 \\
\hline
\end{tabular}

* Significant correlations with $p \leq 0.05$ after correcting for multiple testing. 


\section{Discussion}

The present study aimed to investigate which e-liquid flavors appeal to which user group, as the relative effect of e-cigarette use on health differs between these groups. We found that the smell of sweet and minty e-liquid flavors was liked equally by all groups, and clearly more than tobacco flavors. Furthermore, the smell of the smell of tobacco-flavored e-liquids was less disliked by adult smokers than by adolescent and young adult non-smokers, although differences in mean ratings were small.

\section{Liking of minty and sweet e-liquid flavors}

Not surprisingly, liking ratings for e-liquids with a minty and sweet flavor label were relatively high. Sweet flavors are universally liked, as people have an innate preference for sweet taste ${ }^{43}$. These findings are also in line with previous vaping studies, in which liking of e-liquid flavors significantly positively correlated with sweetness and coolness ${ }^{22,36}$. Similar results were found within our data: the e-liquids with peppermint and menthol flavors received the highest ratings for familiarity and e-liquids with sweet flavor labels were rated highest on sweetness, and we found strong positive associations between liking and familiarity, and between liking and sweetness, respectively. The fact that we used nicotine-free e-liquids and found similar results compared to previous studies using nicotine-containing e-liquids ${ }^{22,36}$ may imply that (sweet and minty) flavors also independently of nicotine contribute to reward from e-cigarettes.

A review from Hoffman et al. ${ }^{44}$ about general flavor preferences showed that preference for sweet taste is highest in children and decreases with age. Therefore, in our study, we expected that the group of adolescents would like the smell of e-liquids with a sweet flavor label more than the group of young adults and adults. Although the correlation between liking and sweetness was significantly stronger among adolescents, we found no significant differences between the user groups in their liking ratings for the typically sweet e-liquids. A reason for this may be that we included adolescents from 16 years old and not children of a younger age. As particularly children have a strong preference for sweet flavors in comparison to adults ${ }^{44,45}$, there may be a difference between children and adults in liking of sweet e-liquid flavors. Further research on this topic with children between 12 and 16 years old would be interesting to determine whether liking of sweet e-liquid flavors is even higher in this group. As the prevalence of e-cigarette use in this age group is concerningly high ${ }^{46,47}$, sensory research in children, although ethically challenging, could provide additional support for regulation of (sweet) e-liquid flavors. This could reduce e-cigarette attractiveness, use, and thus health risks among young people who would otherwise not smoke.

\section{Disliking of tobacco-flavored and other non-sweet e-liquids}

Non-sweet e-liquid flavors, such as whiskey, tobacco, and espresso were disliked the most within all user groups. These type of flavors received the highest ratings for bitterness and irritation, which is supported by the fact that people have an innate aversion to the taste of bitter ${ }^{43}$. This is consistent with the negative correlation between liking and bitterness, which was even stronger in adolescents than both groups of young adults and adults, and between liking and irritation that we found in this study across all flavors. Previous vaping studies found that liking negatively 
correlated with bitterness and harshness/irritation, and suggested these sensory effects to be most likely caused by nicotine ${ }^{22,35,36}$. In the current study, nicotine-free e-liquids were used and similar results were found, which supports the use of smelling as an approach to hedonically assess e-liquid flavors ${ }^{40}$.

We found significant differences in liking of tobacco-flavored e-liquids between the user groups. Tobacco-flavored e-liquids were less disliked by adult smokers than by adolescent and young adult non-smokers, even though differences in mean ratings were small. These betweengroup differences are in line with previous findings that smokers are more interested in trying an e-liquid with tobacco flavor as compared to (young) non-smokers ${ }^{23,27,29,31,32,48}$. Similarly, in the current study, smokers reported far more often to be interested in trying a tobacco-flavored e-liquid than the groups of non-smokers (see Table 8.2). For these reasons, and due to their learned associations between tobacco flavor and perceived consequences of nicotine consumption ${ }^{49}$, we expected the group of smokers to actually like tobacco-flavored e-liquids. However, their mean hedonic ratings for these products' flavors ranged from "Dislike" (cigar) to "Neither Like nor Dislike" (American blend). In addition, even though they reported to be interested in trying tobacco flavors more often than the other groups, smokers were more interested in other flavors (fruit and menthol/mint). In line with this, literature showed that also non-tobacco flavors, such as sweet flavors, considerably appeal to (young) adult smokers ${ }^{29,32,37,50}$, and that fruit and other sweet flavors are actually most popular among e-cigarette users (who are often former smokers) 15,16,19-21,23-25. Thus, it can be questioned whether (former) smokers actually like the tobaccoflavored e-liquids that are currently available on the market. In fact, since current and former smokers often seem to transition from using tobacco to using sweet e-cigarette flavors over time $18,27,51$, it is possible that they used tobacco flavors at initiation primarily because they expected that vaping those flavors would simulate the smoking experience best, while vaping tobacco flavors may actually not sufficiently represent smoking a regular cigarette in terms of flavor and/or other sensory aspects. Further research is needed to find a likeable tobacco flavor for e-cigarettes to facilitate smoking cessation in countries where other flavors than tobacco are not allowed.

\section{Flavor perception in e-liquids vs. other products}

Participants were not informed about the flavor quality (i.e., flavor name) of the e-liquids when performing the sensory test, which causes their familiarity and liking ratings to be solely based on the e-liquids' odors. Sensory ratings for odor familiarity did not correlate with how often participants reported to eat, drink, or use a (often food) product with that flavor in daily life according to the survey questions. In addition, the correlation between participants' sensory assessment of flavor liking by means of smelling the e-liquids and their answers to the survey question how much they like another (food) product with the same flavor was weak. This collectively implies that perception may differ between flavors in e-liquids and the same flavor in another (food) product, and that people may not per se like the same flavors in e-liquids as they like in food. A reason for this may be that the flavor name of e-liquids does not always represent the "real" flavor as we know from another (food) product. For example, an e-liquid labeled as having banana flavor may taste more like banana candy; in this case, we would ask how much participants like and how often they eat banana (and not banana candy). This is similar to 
our hypothesis that tobacco-flavored e-liquids may not represent the flavor of a real cigarette. Moreover, there is not just one e-liquid labeled as having, for example, a strawberry flavor, but there are multiple strawberry-flavored e-liquids available ${ }^{5}$ that each have different chemical flavor compositions ${ }^{52}$. These products may thus be perceived as more or less similar to the actual fruit and may be liked differently. Taken together, more research is needed to better understand the relation between flavor perception and liking in e-cigarettes compared to other products such as food, and how this differs between user groups (e.g., smokers, non-smokers, youth, adults).

\section{Implications}

By far, sweet and minty e-liquid flavors were liked more than tobacco flavors in all groups. This suggests that if countries would decide to ban all e-liquid flavors except tobacco, this will likely reduce attractiveness of e-cigarettes for all user groups. This may reduce and prevent further e-cigarette use and associated health risks among young non-smokers, thereby improving public health. It is unknown whether tobacco flavors would be sufficiently attractive for smokers to permanently switch towards e-cigarette use, thereby improving their health. As smoking cessation and expected health benefits are still the most important reasons for smokers to start using e-cigarettes ${ }^{53}$, they might continue doing so even if they somewhat dislike the e-liquid flavors available on the market. Another possibility is that this would cause former smokers to quit using e-cigarettes, which would further improve their health (unless they start smoking again). Future research on the effect of banning all e-liquid flavors except tobacco on (former) smokers is needed.

\section{Strengths and limitations}

Worldwide, this study was the first sensory study on e-liquid flavors that included adolescent non-smokers, thereby contributing to a better understanding of e-liquid flavor liking in this, from a public health point of view, highly interesting user group. Furthermore, we standardized odor intensity in pilot experiments, as sensory intensity is known to influence liking ${ }^{54}$. This resulted in mean ratings for perceived odor intensity across all users ranging from 44.5 to 73.0 , which is not too weak nor too strong.

Some limitations of this study should be noted. Firstly, we had difficulties recruiting participants due to the COVID-19 outbreak, hence the aimed sample size was not met for the groups of adolescent and young adult non-smokers. However, based on our initial sample size calculations, the power value associated with the final sample size was $>75 \%$ for both groups, which we considered acceptable. Moreover, the between-group comparisons resulted in similar outcomes when analyzing the groups separately and combined into one group of non-smokers (n $=83$ ). Secondly, we used nicotine-free e-liquids and an orthonasal smelling approach. Although we previously found a strong correlation $(R=0.84)$ between orthonasal smelling and vaping in hedonic assessment of nicotine-free e-liquid flavors ${ }^{6}$, the role of nicotine in (dis)liking of e-liquid flavors through its taste and chemesthetic sensations was not covered in this study due to ethical reasons, as we included nicotine-naïve individuals (non-smokers) and individuals under legal age for e-cigarette use (adolescents). 


\section{Conclusions}

We found that e-liquids with sweet and minty flavors were liked equally, and both clearly more than tobacco flavors, by all groups of potential e-cigarette users (i.e., adolescent non-smokers, young adult non-smokers, and adult smokers). Tobacco-flavored e-liquids were slightly less disliked by adult smokers than by the two groups of young non-smokers. Furthermore, in general, sweet and familiar flavors positively influence liking of e-cigarettes, while flavors with high levels of perceived bitterness, irritation, and a strong intensity negatively impact the liking of e-cigarettes. These results suggest that if regulators decide to ban all e-liquid flavors except tobacco, this will likely reduce e-cigarette appeal for all user groups; potentially more for young non-smokers than adult smokers. Finally, discrepancies between sensory liking and familiarity of e-liquid flavors, and liking and use of other products with the same flavor in daily life imply that perception of e-liquid flavors may not always be the same as perception of other products with the same flavor name (e.g., foods or tobacco cigarettes). 


\section{Appendix}

Appendix Table A8.1: Survey questions and answer options that were combined, recoded, and presented into different answer categories (see Table 8.2 in the main text).

\begin{tabular}{|c|c|c|c|}
\hline Survey item & Question & Answer options & Recoding scheme \\
\hline \multirow{7}{*}{$\begin{array}{l}\text { Education } \\
\text { level }\end{array}$} & \multirow{7}{*}{$\begin{array}{l}\text { What is your } \\
\text { highest degree } \\
\text { of education? }\end{array}$} & $1=$ Primary school & Low $=1+2$ \\
\hline & & \multirow{3}{*}{$\begin{array}{l}2=\text { Pre-vocational secondary education (in Dutch: } \\
\text { VMBO), senior general secondary education } \\
\text { (HAVO), middle school pre-university education } \\
\text { (VWO onderbouw), secondary vocational } \\
\text { education level } 1 \text { (MBO1) }\end{array}$} & Middle $=3$ \\
\hline & & & High $=4+5$ \\
\hline & & & \\
\hline & & $\begin{array}{l}3=\text { High school or secondary vocational } \\
\text { education }\end{array}$ & \\
\hline & & $4=$ Bachelor's degree & \\
\hline & & $5=$ Master's or doctorate degree & \\
\hline \multirow{7}{*}{$\begin{array}{l}\text { Intention to } \\
\text { start vaping }\end{array}$} & \multirow{7}{*}{$\begin{array}{l}\text { To what extent } \\
\text { do you intend to } \\
\text { start vaping? }\end{array}$} & 1 = I don’t want to start vaping & No $=1$ \\
\hline & & $2=$ I want to start, but don't know yet when & Low $=2+3$ \\
\hline & & $3=\mathrm{I}$ want to start, hopefully soon & High $=4+5+6$ \\
\hline & & $4=\mathrm{I}$ really want to start, but don't know yet when & Don't know $=7$ \\
\hline & & $\begin{array}{l}5=\text { I really want to start, planned to start in next } \\
\text { three months }\end{array}$ & \\
\hline & & $\begin{array}{l}6=\text { I really want to start, planned to start in } \\
\text { coming month }\end{array}$ & \\
\hline & & 7 = I don't know & \\
\hline \multirow{8}{*}{$\begin{array}{l}\text { Intention to } \\
\text { quit smoking }\end{array}$} & \multirow{8}{*}{$\begin{array}{l}\text { To what extent } \\
\text { do you intend } \\
\text { to quit smoking } \\
\text { in the coming } 6 \\
\text { months? }\end{array}$} & $1=\mathrm{I}$ don't want to quit & $\mathrm{No}=1+2$ \\
\hline & & $2=$ I think I should quit, but don't want to & Low $=3+4$ \\
\hline & & 3 = I want to quit, but don't know yet when & High $=5+6+7$ \\
\hline & & $4=$ I want to quit, hopefully soon & Don't know $=8$ \\
\hline & & $5=$ I really want to quit, but don't know yet when & \\
\hline & & $\begin{array}{l}6=\text { I really want to quit, planned to quit in next } \\
\text { three months }\end{array}$ & \\
\hline & & $\begin{array}{l}7=\text { I really want to quit, planned to quit in } \\
\text { coming month }\end{array}$ & \\
\hline & & $8=\mathrm{I}$ don’t know & \\
\hline
\end{tabular}

Questions not mentioned in the current table were presented in the main text with their actual answer options (no recoding needed). 
Appendix Table A8.2: Mean liking ratings $( \pm \mathrm{SE})$ for individual e-liquids $(\mathrm{n}=30)$ and for 4 groups of products with similar flavors (for classification, see Table A8.4), assessed by adolescent non-smokers (n $=41)$, young adult non-smokers $(n=42)$, and adult smokers $(n=56)$ on a 9-point labeled hedonic scale.

\begin{tabular}{lrrrr}
\hline & $\begin{array}{c}\text { Adolescent non- } \\
\text { smokers }\end{array}$ & $\begin{array}{c}\text { Young adult } \\
\text { non-smokers }\end{array}$ & Adult smokers \\
\hline Peppermint & $6.9 \pm 0.3$ & $6.7 \pm 0.3$ & $6.7 \pm 0.2$ \\
Wine gum & $6.9 \pm 0.3$ & $6.7 \pm 0.3$ & $6.4 \pm 0.3$ \\
Menthol & $6.9 \pm 0.3$ & $6.7 \pm 0.3$ & $6.1 \pm 0.3$ \\
Bubblegum & $6.4 \pm 0.3$ & $6.2 \pm 0.3$ & $6.7 \pm 0.2$ \\
Anise & $6.4 \pm 0.2$ & $5.7 \pm 0.3$ & $6.3 \pm 0.2$ \\
Watermelon & $6.6 \pm 0.3$ & $6.0 \pm 0.3$ & $5.9 \pm 0.3$ \\
Citrus fruits & $6.1 \pm 0.3$ & $6.2 \pm 0.3$ & $6.0 \pm 0.2$ \\
Raspberry & $5.8 \pm 0.3$ & $5.9 \pm 0.3$ & $6.4 \pm 0.2$ \\
Mojito & $6.2 \pm 0.3$ & $5.9 \pm 0.3$ & $6.0 \pm 0.3$ \\
Cola & $6.4 \pm 0.3$ & $5.9 \pm 0.3$ & $5.7 \pm 0.3$ \\
Energy drink & $6.2 \pm 0.3$ & $5.7 \pm 0.3$ & $5.9 \pm 0.2$ \\
Vanilla & $5.1 \pm 0.3$ & $5.8 \pm 0.3$ & $5.9 \pm 0.2$ \\
Jasmine tea & $5.3 \pm 0.3$ & $5.3 \pm 0.3$ & $5.8 \pm 0.2$ \\
Lavender & $5.4 \pm 0.3$ & $5.8 \pm 0.3$ & $5.2 \pm 0.3$ \\
Pineapple & $5.7 \pm 0.3$ & $5.4 \pm 0.3$ & $5.3 \pm 0.3$ \\
Unflavored & $5.2 \pm 0.2$ & $5.3 \pm 0.2$ & $4.9 \pm 0.1$ \\
Syrup waffle & $4.6 \pm 0.3$ & $5.2 \pm 0.4$ & $4.5 \pm 0.3$ \\
Cheesecake & $4.0 \pm 0.3$ & $4.8 \pm 0.4$ & $4.6 \pm 0.3$ \\
Espresso & $3.9 \pm 0.3$ & $4.1 \pm 0.3$ & $4.8 \pm 0.3$ \\
Caramel & $3.9 \pm 0.3$ & $4.6 \pm 0.3$ & $4.4 \pm 0.3$ \\
American blend * & $3.5^{\mathrm{A}} \pm 0.3$ & $3.8^{\mathrm{A}} \pm 0.3$ & $4.9^{\mathrm{B}} \pm 0.2$ \\
Clove & $3.7 \pm 0.3$ & $3.6 \pm 0.3$ & $4.3 \pm 0.3$ \\
Oriental * & $3.0^{\mathrm{A}} \pm 0.2$ & $4.3^{\mathrm{B}} \pm 0.3$ & $4.3^{\mathrm{B}} \pm 0.3$ \\
Tobacco_b & $3.2 \pm 0.3$ & $3.6 \pm 0.2$ & $4.1 \pm 0.3$ \\
Hazelnut & $3.1 \pm 0.3$ & $3.8 \pm 0.3$ & $3.6 \pm 0.3$ \\
Tobacco_a & $3.2 \pm 0.2$ & $3.2 \pm 0.2$ & $3.7 \pm 0.3$ \\
Peanut & $2.9 \pm 0.3$ & $3.5 \pm 0.3$ & $3.5 \pm 0.3$ \\
Tobacco_c & $2.9 \pm 0.3$ & $2.8 \pm 0.3$ & $3.2 \pm 0.3$ \\
Cigar & $2.9 \pm 0.3$ & $2.9 \pm 0.3$ & $3.1 \pm 0.2$ \\
Whiskey & $2.4 \pm 0.3$ & $2.1 \pm 0.2$ & $2.4 \pm 0.2$ \\
\hline & & & & \\
\hline
\end{tabular}

Groups of products with similar flavors

\begin{tabular}{lrlrlrl}
\hline Tobacco flavors * & $3.1^{\mathrm{A}}$ & \pm 0.3 & $3.4^{\mathrm{B}}$ & \pm 0.3 & $3.9^{\mathrm{C}}$ & \pm 0.2 \\
Minty flavors & 6.9 & \pm 0.3 & 6.7 & \pm 0.3 & 6.4 & \pm 0.2 \\
Other non-sweet flavors * & $3.2^{\mathrm{A}}$ & \pm 0.3 & $3.4^{\mathrm{AB}}$ & \pm 0.3 & $3.7^{\mathrm{B}}$ & \pm 0.3 \\
Sweet flavors & 5.7 & \pm 0.3 & 5.7 & \pm 0.3 & 5.7 & \pm 0.3 \\
\hline
\end{tabular}

Products were ranked from highest to lowest mean liking score across all users $(\mathrm{n}=139)$.

The same data are visualized in Figure 8.1 (main text).

* Different letters in superscript indicate significant differences in a row (i.e., between user groups). 
Appendix Table A8.3: Mean familiarity ratings $( \pm \mathrm{SE})$ for individual e-liquids $(\mathrm{n}=30)$ and for 4 groups of products with similar flavors (for classification, see Table A8.4), assessed by adolescent non-smokers ( $\mathrm{n}$ $=41$ ), young adult non-smokers $(n=42)$, and adult smokers $(n=56)$ on a 100-unit Visual Analog Scale.

\begin{tabular}{|c|c|c|c|c|c|c|}
\hline \multirow[b]{2}{*}{ Peppermint } & \multicolumn{2}{|c|}{$\begin{array}{l}\text { Adolescent non- } \\
\text { smokers }\end{array}$} & \multicolumn{2}{|c|}{$\begin{array}{l}\text { Young adult } \\
\text { non-smokers }\end{array}$} & \multicolumn{2}{|c|}{ Adult smokers } \\
\hline & 82.9 & \pm 3.3 & 89.7 & \pm 1.5 & 80.1 & \pm 3.3 \\
\hline Menthol & 83.1 & \pm 2.6 & 81.2 & \pm 3.2 & 72.2 & \pm 3.7 \\
\hline Anise & 76.6 & \pm 4.0 & 73.2 & \pm 3.4 & 72.0 & \pm 3.3 \\
\hline Watermelon & 78.9 & \pm 3.9 & 70.8 & \pm 3.5 & 59.5 & \pm 4.2 \\
\hline Energy drink & 68.6 & \pm 4.6 & 69.7 & \pm 3.4 & 66.3 & \pm 3.6 \\
\hline Mojito & 65.1 & \pm 4.3 & 67.8 & \pm 3.9 & 66.3 & \pm 3.4 \\
\hline Wine gum & 76.6 & \pm 3.6 & 64.3 & \pm 3.4 & 58.9 & \pm 3.5 \\
\hline Cola & 71.7 & \pm 3.4 & 62.7 & \pm 3.9 & 63.3 & \pm 3.9 \\
\hline Espresso & 67.4 & \pm 4.8 & 64.1 & \pm 4.6 & 58.3 & \pm 4.6 \\
\hline Bubblegum & 68.7 & \pm 3.8 & 61.0 & \pm 3.5 & 58.6 & \pm 3.6 \\
\hline Lavender & 61.7 & \pm 4.5 & 63.4 & \pm 4.1 & 52.3 & \pm 3.9 \\
\hline Citrus fruit & 60.2 & \pm 3.9 & 55.5 & \pm 3.7 & 51.5 & \pm 3.7 \\
\hline Raspberry & 58.9 & \pm 4.8 & 54.0 & \pm 4.1 & 52.1 & \pm 3.7 \\
\hline Jasmine tea & 47.4 & \pm 4.2 & 53.6 & \pm 3.8 & 59.6 & \pm 3.2 \\
\hline Syrup waffle & 59.3 & \pm 4.4 & 58.8 & \pm 3.9 & 46.0 & \pm 4.0 \\
\hline Pineapple & 61.1 & \pm 4.5 & 53.3 & \pm 3.9 & 48.5 & \pm 4.1 \\
\hline Vanilla & 48.4 & \pm 4.9 & 46.0 & \pm 4.2 & 48.1 & \pm 4.1 \\
\hline Hazelnut & 40.1 & \pm 4.7 & 51.3 & \pm 5.2 & 47.7 & \pm 4.1 \\
\hline Caramel & 41.3 & \pm 4.4 & 49.6 & \pm 4.3 & 47.9 & \pm 4.2 \\
\hline Clove & 42.9 & \pm 4.9 & 43.8 & \pm 4.8 & 49.9 & \pm 4.5 \\
\hline Cheesecake & 41.8 & \pm 4.7 & 50.2 & \pm 4.0 & 44.8 & \pm 3.9 \\
\hline Peanut & 44.3 & \pm 4.8 & 51.0 & \pm 4.1 & 36.4 & \pm 3.8 \\
\hline American blend & 30.3 & \pm 3.9 & 33.3 & \pm 3.8 & 47.0 & \pm 4.1 \\
\hline Oriental & 30.7 & \pm 3.8 & 35.8 & \pm 4.1 & 41.8 & \pm 4.1 \\
\hline Whiskey & 34.4 & \pm 5.0 & 34.7 & \pm 3.9 & 39.6 & \pm 4.0 \\
\hline Tobacco_b & 31.4 & \pm 3.9 & 33.5 & \pm 3.8 & 38.5 & \pm 3.8 \\
\hline Tobacco_a & 30.3 & \pm 4.1 & 29.8 & \pm 3.2 & 36.6 & \pm 4.0 \\
\hline Tobacco_c & 25.6 & \pm 3.8 & 28.9 & \pm 3.7 & 34.8 & \pm 3.8 \\
\hline Cigar & 32.9 & \pm 3.0 & 27.0 & \pm 3.0 & 30.9 & \pm 3.3 \\
\hline Unflavored & 21.3 & \pm 3.2 & 21.5 & \pm 4.0 & 18.9 & \pm 3.6 \\
\hline \multicolumn{7}{|c|}{ Groups of products with similar flavors } \\
\hline Tobacco flavors & 30.2 & \pm 3.7 & 31.4 & \pm 3.6 & 38.3 & \pm 3.8 \\
\hline Minty flavors & 83.0 & \pm 2.9 & 85.5 & \pm 2.3 & 76.1 & \pm 3.5 \\
\hline Other non-sweet flavors & 45.8 & \pm 4.9 & 49.0 & \pm 4.5 & 46.4 & \pm 4.2 \\
\hline Sweet flavors & 61.6 & \pm 4.2 & 59.6 & \pm 3.8 & 56.0 & \pm 3.8 \\
\hline
\end{tabular}

Products were ranked from highest to lowest mean liking score across all users $(n=139)$.

Familiarity did not significantly differ between user groups, for any of the e-liquids. 
Appendix Table A8.4: Mean sweetness ratings $( \pm \mathrm{SE})$ for individual e-liquids $(\mathrm{n}=30)$ and for 4 groups of products with similar flavors, assessed by adolescent non-smokers $(n=41)$, young adult non-smokers $(n=42)$, and adult smokers $(n=56)$ on a 100-unit Visual Analog Scale.

\begin{tabular}{|c|c|c|c|c|c|c|}
\hline \multirow[b]{2}{*}{ Energy drink } & \multicolumn{2}{|c|}{$\begin{array}{c}\text { Adolescent non- } \\
\text { smokers }\end{array}$} & \multicolumn{2}{|c|}{$\begin{array}{c}\text { Young adult } \\
\text { non-smokers }\end{array}$} & Adult smokers & \multirow{2}{*}{$\begin{array}{c}\text { Assigned group } \\
\text { Sweet }\end{array}$} \\
\hline & 78.6 & \pm 3.2 & 83.6 & \pm 2.4 & $77.4 \pm 2.7$ & \\
\hline Wine gum & 81.9 & \pm 2.7 & 77.7 & \pm 2.8 & $72.4 \pm 2.8$ & Sweet \\
\hline Bubblegum & 78.5 & \pm 3.0 & 74.3 & \pm 3.0 & $71.2 \pm 2.8$ & Sweet \\
\hline Watermelon & 79.1 & \pm 3.2 & 76.1 & \pm 2.8 & $63.4 \pm 3.7$ & Sweet \\
\hline Raspberry & 75.7 & \pm 3.2 & 69.2 & \pm 3.4 & $69.4 \pm 2.7$ & Sweet \\
\hline Citrus fruit & 75.8 & \pm 2.4 & 70.1 & \pm 3.2 & $66.3 \pm 3.3$ & Sweet \\
\hline Pineapple & 70.7 & \pm 3.5 & 70.4 & \pm 3.0 & $63.1 \pm 3.7$ & Sweet \\
\hline Anise & 62.5 & \pm 3.4 & 57.2 & \pm 2.9 & $62.0 \pm 3.1$ & Sweet \\
\hline Cheesecake & 55.4 & \pm 4.1 & 62.0 & \pm 4.1 & $52.4 \pm 3.5$ & Sweet \\
\hline Vanilla & 54.3 & \pm 4.1 & 56.5 & \pm 3.9 & $55.1 \pm 2.9$ & Sweet \\
\hline Caramel & 46.9 & \pm 4.0 & 64.4 & \pm 3.7 & $51.2 \pm 3.7$ & Sweet \\
\hline Syrup waffle & 51.8 & \pm 4.3 & 53.8 & \pm 4.2 & $53.6 \pm 3.9$ & Sweet \\
\hline Cola & 56.3 & \pm 4.1 & 44.9 & \pm 3.9 & $52.0 \pm 3.5$ & Sweet \\
\hline Jasmine tea & 47.9 & \pm 3.7 & 43.0 & \pm 4.0 & $57.8 \pm 3.4$ & Sweet \\
\hline Mojito & 52.2 & \pm 3.4 & 44.8 & \pm 3.5 & $46.9 \pm 3.3$ & Sweet \\
\hline Lavender & 50.2 & \pm 3.2 & 41.0 & \pm 3.3 & $44.0 \pm 3.5$ & Sweet \\
\hline Peppermint & 51.0 & \pm 3.9 & 37.2 & \pm 3.5 & $42.8 \pm 3.7$ & Minty \\
\hline Menthol & 53.0 & \pm 4.0 & 35.6 & \pm 3.9 & $37.5 \pm 3.6$ & Minty \\
\hline Hazelnut & 33.0 & \pm 3.8 & 42.4 & \pm 4.1 & $37.9 \pm 3.6$ & Non-sweet \\
\hline Tobacco_b & 41.2 & \pm 4.4 & 40.3 & \pm 3.9 & $31.7 \pm 3.6$ & Tobacco \\
\hline Oriental & 34.0 & \pm 3.9 & 39.6 & \pm 3.8 & $37.4 \pm 3.3$ & Tobacco \\
\hline Peanut & 33.5 & \pm 4.5 & 40.4 & \pm 3.9 & $34.8 \pm 3.8$ & Non-sweet \\
\hline Clove & 34.1 & \pm 3.8 & 33.2 & \pm 3.3 & $39.4 \pm 3.5$ & Non-sweet \\
\hline American blend & 30.9 & \pm 3.5 & 35.4 & \pm 4.0 & $37.7 \pm 3.6$ & Tobacco \\
\hline Tobacco_a & 27.8 & \pm 3.4 & 34.8 & \pm 3.4 & $34.1 \pm 3.8$ & Tobacco \\
\hline Espresso & 24.2 & \pm 3.2 & 36.1 & \pm 3.8 & $33.8 \pm 3.5$ & Non-sweet \\
\hline Cigar & 24.2 & \pm 3.2 & 30.3 & \pm 3.4 & $31.8 \pm 3.5$ & Tobacco \\
\hline Tobacco_c & 21.0 & \pm 3.1 & 27.1 & \pm 3.1 & $28.7 \pm 3.4$ & Tobacco \\
\hline Whiskey & 19.1 & \pm 2.7 & 27.6 & \pm 3.0 & $25.6 \pm 3.5$ & Non-sweet \\
\hline Unflavored & 29.3 & \pm 4.1 & 22.9 & \pm 3.9 & $17.2 \pm 3.0$ & N/A \\
\hline
\end{tabular}

Groups of products with similar flavors

\begin{tabular}{|c|c|c|c|}
\hline Tobacco flavors & $29.8 \pm 3.6$ & $34.6 \pm 3.6$ & $33.5 \pm 3.5$ \\
\hline Minty flavors & $52.0 \pm 3.9$ & $36.4 \pm 3.7$ & $40.2 \pm 3.7$ \\
\hline Other non-sweet flavors & $28.8 \pm 3.6$ & $35.9 \pm 3.6$ & $34.3 \pm 3.6$ \\
\hline Sweet flavors & $63.6 \pm 3.5$ & $61.8 \pm 3.4$ & $59.9 \pm 3.3$ \\
\hline
\end{tabular}

Products were ranked from highest to lowest mean liking score across all users $(n=139)$. Sweetness did not significantly differ between user groups, for any of the e-liquids. Categorization of the e-liquids (excluding unflavored) into 4 groups was based on similar flavor types and sweetness ratings (final column). 


\section{References}

1. Rozin P, Fallon A. The psychological categorization of foods and non-foods: A preliminary taxonomy of food rejections. Appetite. 1980;1(3):193-201.

2. Steptoe A, Pollard TM, Wardle J. Development of a measure of the motives underlying the selection of food: the food choice questionnaire. Appetite. 1995;25(3):267-284.

3. Megerdichian CL, Rees VW, Wayne GF, Connolly GN. Internal tobacco industry research on olfactory and trigeminal nerve response to nicotine and other smoke components. Nicotine Tob Res. 2007;9(11):1119-1129.

4. Ferris Wayne G, Connolly GN. Application, function, and effects of menthol in cigarettes: a survey of tobacco industry documents. Nicotine Tob Res. 2004;6 Suppl 1:S43-54.

5. Havermans A, Krüsemann EJZ, Pennings J, de Graaf K, Boesveldt S, Talhout R. Nearly 20000 e-liquids and 250 unique flavour descriptions: an overview of the Dutch market based on information from manufacturers. Tob Control. 2019:tobaccocontrol-2019-055303.

6. Krüsemann EJZ, Boesveldt S, de GraafK, Talhout R. An E-Liquid Flavor Wheel: A Shared Vocabulary Based on Systematically Reviewing E-Liquid Flavor Classifications in Literature. Nicotine Tob Res. 2019;21(10):1310-1319.

7. Goniewicz ML, Knysak J, Gawron M, et al. Levels of selected carcinogens and toxicants in vapour from electronic cigarettes. Tob Control. 2014;23(2):133-139.

8. Schmidt S. Vaper, Beware: The Unique Toxicological Profile of Electronic Cigarettes. Environ Health Perspect. 2020;128(5):52001.

9. Hilton S, Weishaar H, Sweeting H, Trevisan F, Katikireddi SV. E-cigarettes, a safer alternative for teenagers? A UK focus group study of teenagers' views. BMJ Open. 2016;6(11):e013271.

10. Bold KW, Kong G, Cavallo DA, Camenga DR, Krishnan-Sarin S. Reasons for Trying E-cigarettes and Risk of Continued Use. Pediatrics. 2016;138(3).

11. Kong G, Morean ME, Cavallo DA, Camenga DR, Krishnan-Sarin S. Reasons for Electronic Cigarette Experimentation and Discontinuation Among Adolescents and Young Adults. Nicotine Tob Res. 2015;17(7):847-854.

12. Ambrose BK, Day HR, Rostron B, et al. Flavored Tobacco Product Use Among US Youth Aged 1217 Years, 2013-2014. JAMA. 2015;314(17):1871-1873.

13. Soneji S, Barrington-Trimis JL, Wills TA, et al. Association Between Initial Use of e-Cigarettes and Subsequent Cigarette Smoking Among Adolescents and Young Adults: A Systematic Review and Meta-analysis. JAMA Pediatr. 2017;171(8):788-797.

14. Foley AM. A review of the e-cigarette debate. J Vasc Nurs. 2019;37(2):153-155.

15. Berg CJ. Preferred flavors and reasons for e-cigarette use and discontinued use among never, current, and former smokers. Int J Public Health. 2016;61(2):225-236.

16. Chen Z, Zeng DD. Mining online e-liquid reviews for opinion polarities about e-liquid features. BMC Public Health. 2017;17(1):633.

17. Dawkins L, Turner J, Roberts A, Soar K. 'Vaping' profiles and preferences: an online survey of electronic cigarette users. Addiction. 2013;108(6):1115-1125.

18. Farsalinos KE, Romagna G, Tsiapras D, Kyrzopoulos S, Spyrou A, Voudris V. Impact of flavour variability on electronic cigarette use experience: An internet survey. Int J Environ Res Public Health. 2013;10(12):7272-7282.

19. Goldenson NI, Kirkpatrick MG, Barrington-Trimis JL, et al. Effects of sweet flavorings and nicotine 
on the appeal and sensory properties of e-cigarettes among young adult vapers: Application of a novel methodology. Drug Alcohol Depend. 2016;168:176-180.

20. Harrell MB, Weaver SR, Loukas A, et al. Flavored e-cigarette use: Characterizing youth, young adult, and adult users. Prev Med Reports. 2017;5:33-40.

21. Huang LL, Baker HM, Meernik C, Ranney LM, Richardson A, Goldstein AO. Impact of nonmenthol flavours in tobacco products on perceptions and use among youth, young adults and adults: a systematic review. Tob Control. 2017;26(6):709-719.

22. Kim H, Lim J, Buehler SS, et al. Role of sweet and other flavours in liking and disliking of electronic cigarettes. Tob Control. 2016;25(Suppl 2):ii55-ii61.

23. Shiplo S, Czoli CD, Hammond D. E-cigarette use in Canada: Prevalence and patterns of use in a regulated market. BMJ Open. 2015;5(8).

24. Tackett AP, Lechner WV, Meier E, et al. Biochemically verified smoking cessation and vaping beliefs among vape store customers. Addiction. 2015;110(5):868-874.

25. Wang L, Zhan Y, Li Q, Zeng DD, Leischow SJ, Okamoto J. An Examination of Electronic Cigarette Content on Social Media: Analysis of E-Cigarette Flavor Content on Reddit. Int J Environ Res Public Health. 2015;12(11):14916-14935.

26. Yingst JM, Veldheer S, Hammett E, Hrabovsky S, Foulds J. A Method for Classifying User-Reported Electronic Cigarette Liquid Flavors. Nicotine Tob Res. 2017;19(11):1381-1385.

27. Romijnders KA, Krusemann EJ, Boesveldt S, Graaf K, Vries H, Talhout R. E-Liquid Flavor Preferences and Individual Factors Related to Vaping: A Survey among Dutch Never-Users, Smokers, Dual Users, and Exclusive Vapers. Int J Environ Res Public Health. 2019;16(23).

28. Zare S, Nemati M, Zheng Y. A systematic review of consumer preference for e-cigarette attributes: Flavor, nicotine strength, and type. PLoS One. 2018;13(3):e0194145.

29. Krishnan-Sarin S, Morean ME, Camenga DR, Cavallo DA, Kong G. E-cigarette use among high school and middle school adolescents in Connecticut. Nicotine Tob Research. 2015;17(7):810-818.

30. Morean ME, Butler ER, Bold KW, et al. Preferring more e-cigarette flavors is associated with e-cigarette use frequency among adolescents but not adults. PLoS One. 2018;13(1):e0189015.

31. Czoli CD, Goniewicz M, Islam T, Kotnowski K, Hammond D. Consumer preferences for electronic cigarettes: results from a discrete choice experiment. Tob Control. 2016;25(e1):e30-36.

32. Shiffman S, Sembower MA, Pillitteri JL, Gerlach KK, Gitchell JG. The Impact of Flavor Descriptors on Nonsmoking Teens' and Adult Smokers' Interest in Electronic Cigarettes. Nicotine Tob Res. 2015;17(10):1255-1262.

33. Ford A, MacKintosh AM, Bauld L, Moodie C, Hastings G. Adolescents' responses to the promotion and flavouring of e-cigarettes. Int J Public Health. 2016;61(2):215-224.

34. Pepper JK, Ribisl KM, Brewer NT. Adolescents' interest in trying flavoured e-cigarettes. Tob Control. 2016;25(Suppl 2):ii62-ii66.

35. Pullicin AJ, Kim H, Brinkman MC, Buehler SS, Clark PI, Lim J. Impacts of Nicotine and Flavoring on the Sensory Perception of E-Cigarette Aerosol. Nicotine Tob Res. 2020;22(5):806-813.

36. Mead EL, Duffy V, Oncken C, Litt MD. E-cigarette palatability in smokers as a function of flavorings, nicotine content and propylthiouracil (PROP) taster phenotype. Addict Behav. 2019;91:37-44.

37. Leventhal AM, Goldenson NI, Barrington-Trimis JL, Pang RD, Kirkpatrick MG. Effects of nontobacco flavors and nicotine on e-cigarette product appeal among young adult never, former, and current smokers. Drug Alcohol Depend. 2019;203:99-106. 
38. Jackson A, Green B, Erythropel HC, et al. Influence of menthol and green apple e-liquids containing different nicotine concentrations among youth e-cigarette users. Exp Clin Psychopharmacol. 2020;10.1037/pha0000368.

39. Rosbrook K, Erythropel HC, DeWinter TM, et al. The effect of sucralose on flavor sweetness in electronic cigarettes varies between delivery devices. PLoS One. 2017;12(10):e0185334.

40. Krusemann EJZ, Wenng FM, Pennings JLA, de Graaf K, Talhout R, Boesveldt S. Sensory Evaluation of E-Liquid Flavors by Smelling and Vaping Yields Similar Results. Nicotine Tob Res. 2020;22(5):798805.

41. Heatherton TF, Kozlowski LT, Frecker RC, Fagerstrom KO. The Fagerstrom Test for Nicotine Dependence: a revision of the Fagerstrom Tolerance Questionnaire. Br J Addict. 1991;86(9):11191127.

42. Benjamini Y, Hochberg Y. Controlling the False Discovery Rate: A Practical and Powerful Approach to Multiple Testing. J Roy Stat Soc B Met. 1995;57(1):289-300.

43. Steiner JE. Human Facial Expressions in Response to Taste and Smell Stimulation. In: Reese HW, Lipsitt LP, eds. Adv Child Dev Behav. 1979;13:257-295.

44. Hoffman AC, Salgado RV, Dresler C, Faller RW, Bartlett C. Flavour preferences in youth versus adults: a review. Tob Control. 2016;25(Suppl 2):ii32-ii39.

45. Zandstra EH, de Graaf C. Sensory perception and pleasantness of orange beverages from childhood to old age. Food Qual Prefer. 1998;9(1-2):5-12.

46. Cullen KA, Gentzke AS, Sawdey MD, et al. e-Cigarette Use Among Youth in the United States, 2019. JAMA. 2019;322(21):2095-2103.

47. Stevens G, van Dorsselaer S, Boer M, et al. HBSC 2017: Gezondheid en welzijn van jongeren in Nederland. 2018; https://hbsc-nederland.nl/wp-content/uploads/2018/09/Rapport-HBSC-2017.pdf.

48. Litt MD, Duffy V, Oncken C. Cigarette smoking and electronic cigarette vaping patterns as a function of e-cigarette flavourings. Tob Control. 2016;25(Suppl 2):ii67-ii72.

49. Benowitz NL. Nicotine addiction. N Engl J Med. 2010;362(24):2295-2303.

50. Shiplo S, Czoli CD, Hammond D. E-cigarette use in Canada: prevalence and patterns of use in a regulated market. BMJ Open. 2015;5(8):e007971.

51. Russell C, McKeganey N, Dickson T, Nides M. Changing patterns of first e-cigarette flavor used and current flavors used by 20,836 adult frequent e-cigarette users in the USA. Harm Reduct J. 2018;15(1):33.

52. Aszyk J, Kubica P, Wozniak MK, Namiesnik J, Wasik A, Kot-Wasik A. Evaluation of flavour profiles in e-cigarette refill solutions using gas chromatography-tandem mass spectrometry. J Chromatogr A. 2018; 1547:86-98.

53. Romijnders K, van Osch L, de Vries H, Talhout R. Perceptions and Reasons Regarding E-Cigarette Use among Users and Non-Users: A Narrative Literature Review. Int J Environ Res Public Health. 2018;15(6).

54. Moskowitz HR. Sensory intensity versus hedonic functions: classical psychophysical approaches. $J$ Food Quality. 1981;5:109-137. 



\section{CHAPTER 9}

General discussion 
230 Chapter 9. General Discussion 
This thesis aimed to investigate the role of flavors in attractiveness of e-cigarettes with respect to different user groups. We analyzed the landscape of available e-liquid flavors, flavoring ingredients in e-liquids, and flavor preferences and liking in different user groups. First, the main results of this thesis are summarized. Next, the thesis topic and implications will be discussed, followed by methodological considerations and recommendations for future research. Lastly, the main conclusions are described.

\section{Main findings}

First, to structure the large amount of e-liquid flavors available, we developed a flavor wheel for consistent categorization of e-liquids based on their marketed flavor descriptions (Chapter 2). This flavor wheel was then used to create an overview of the e-liquids marketed in the Netherlands in 2017. We found that the Dutch e-liquid market comprised nearly 20000 e-liquids in 245 different, mostly sweet, flavors (Chapter 3).

Second, we aimed to identify the most prevalent flavoring ingredients in e-liquids. We found that manufacturers most often added vanillin (sweet, vanilla-like flavor), ethyl maltol (sweet, fruity-caramellic flavor) and ethyl butyrate (ethereal, fruity flavor) to their e-liquids, and we identified several flavorings that were specific to a respective flavor category (Chapter 4). Using the e-liquids' flavoring compositions, we could predict the e-liquids' flavor category with $70 \%$ accuracy. Furthermore, our chemical-analytical study showed that e-liquids most often contained vanillin (sweet, vanilla-like flavor), ethyl butyrate (ethereal, fruity flavor), and cis-3hexenol (fresh, green flavor), and that flavoring compositions were similar in fresh/sweet, warm/ sweet, fresh/cooling, and non-sweet flavor categories, respectively (Chapter 5).

Third, we aimed to determine which flavors (potential) users of e-cigarettes prefer and like the most. We showed that, in the Netherlands, smokers were mostly interested in trying e-cigarettes with a tobacco or menthol/mint flavor, whereas people who had never smoked nor vaped were mostly interested in trying sweet and menthol/mint-flavored e-cigarettes (Chapter 6). Before investigating sensory liking of various tobacco and non-tobacco e-liquid flavors, we demonstrated that the correlation between smelling and vaping for liking of e-liquid flavors was strong, and did not differ between smokers and non-smokers (Chapter 7). Finally, we showed that both sweet and minty e-liquid flavors were liked similarly, and clearly more than tobaccoflavored e-liquids, by all groups of potential e-cigarette users (i.e., adolescent non-smokers, young adult non-smokers, and adult smokers) (Chapter 8). An overview of the findings of this thesis is provided in Table 9.1. 


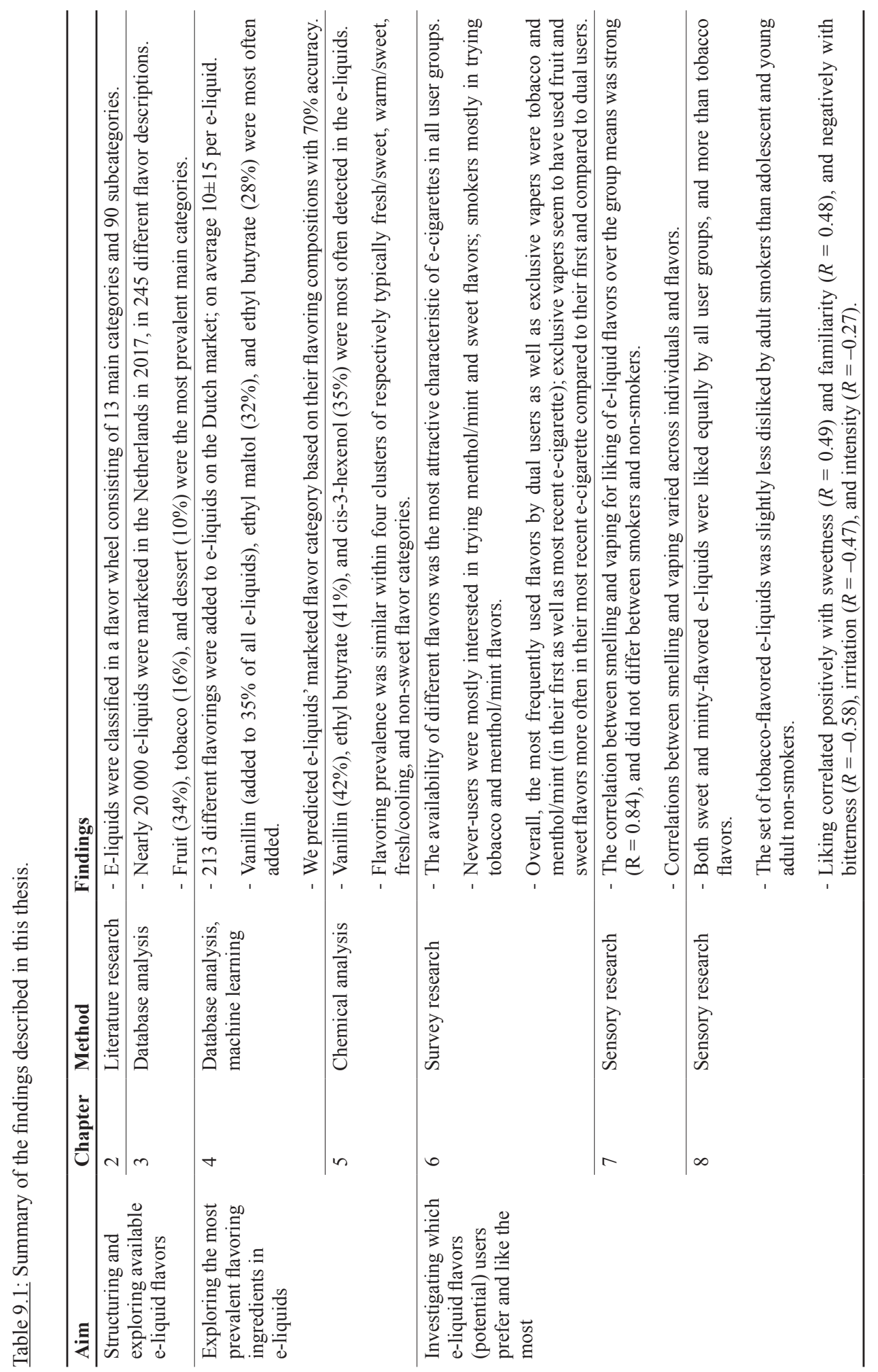




\section{Discussion of the thesis topic and implications}

Discussions of the findings of this thesis are presented in the individual chapters. In this section, we will zoom out and discuss the findings on a higher level in order to address the overall topic of this thesis: the role of flavors in attractiveness of e-cigarettes.

\section{Attractive e-cigarette flavors}

The findings of this thesis consistently show that there is a clear preference for sweet e-liquid flavors: by far the majority of the e-liquids available on the market had a sweet flavor label (Chapter 3); the flavoring ingredients that were most frequently added to (Chapter 4 ) and identified in (Chapter 5) e-liquids have a sweet aroma; sweet flavors were not only most interesting to never-users, but also regularly used by people who vape and not concurrently smoke (Chapter 6); and sweet e-liquid flavors were liked more than tobacco and other non-sweet flavors by adolescent non-smokers, young adult non-smokers, as well as adult smokers (Chapter 8$)$. The popularity of sweet flavors is not surprising, and can be explained by the two mechanisms via which sweet flavors may increase appeal of e-cigarettes. Firstly, sweetness directly contributes to liking, because people have a (innate) preference for sweet tastes ${ }^{1,2}$. This makes sweet flavors universally liked. Secondly, sweetness indirectly contributes to liking by masking harshness and irritation caused by nicotine ${ }^{3}$. Typically, harshness or irritation is negatively correlated, and sweetness is positively correlated with product appeal ratings ${ }^{3}$. These correlations were also found in Chapter 8, even though nicotine-free e-liquids were used.

This thesis showed that also menthol/mint flavors are attractive in e-cigarettes: menthol/ mint flavors raised interest among never-users and smokers (hapter 6); were the most frequently used flavor after tobacco among dual users and exclusive vapers (Chapter 6); and were, similar to sweet flavors, liked most by all target groups of potential e-cigarette users: adolescent nonsmokers, young adult non-smokers, as well as adult smokers (Chapter 8). The contribution of menthol, which is the chemical compound characteristically present in menthol/mint-flavored e-liquids, to sensory perception and appeal of e-cigarettes is quite complex: menthol produces concentration-dependent cooling and irritating sensations at low nicotine concentrations, whereas high menthol concentrations reduce irritation at high nicotine concentrations ${ }^{4,5}$. This implies that menthol could improve e-cigarette appeal directly through its cooling effects at low nicotine concentrations, as well as indirectly by reducing harshness/irritation from high nicotine concentrations ${ }^{6}$. These physiological effects are also the reason why menthol is commonly used as an additive in tobacco cigarettes ${ }^{7}$.

\section{Will banning all e-liquid flavors except tobacco reduce attractiveness of e-cigarettes?}

Over the course of this thesis project, prevalence of e-cigarette use in the Netherlands has decreased ${ }^{8}$. In 2019, 1.6\% of the Dutch adults occasionally used an e-cigarette; nearly all of them were former or current smokers. The percentage of adult vapers who had never smoked was low (less than 2\%). Fortunately, among youth, the prevalence of having ever used an e-cigarette has also decreased in 2019 compared to previous years (data on current daily or occasional use of e-cigarettes are unavailable) ${ }^{9}$. Moreover, Chapter 6 showed that the majority of never-users $(68 \%)$ was not interested in trying an e-cigarette flavor. However, in 2019, still a quarter of 
the secondary school students had ever used an e-cigarette ${ }^{9}$. In addition, never-users reported flavors as the most attractive product factor for e-cigarettes, and still a third of the never-users included in our study was interested in trying an e-cigarette flavor (Chapter 6). The Ministry of Health, Welfare and Sport from the Netherlands aims at a "smoke-free generation" in 2040 (i.e., a generation of children who live in an environment that is free of tobacco), and established several measures to achieve this goal in the National Prevention Agreement ${ }^{10}$. Recently, the State Secretary for Health, Welfare and Sport proposed to extend the National Prevention Agreement towards further restrictions on the attractiveness of e-cigarettes for youth, because he "believes that a smoke-free generation should also be an e-cigarette-free generation" ${ }^{11}$. This is consistent with the advice from the World Health Organization (WHO) to regulate products in order to prevent initiation of e-cigarette use by non-smokers, minors, and vulnerable user groups ${ }^{12}$. Therefore, in 2020, the State Secretary announced a ban in the Netherlands on all e-cigarette flavors except tobacco flavors, which he based, among other things, on the findings described in this thesis ${ }^{11}$. In the same year, the United States Food and Drug Administration (US FDA) implemented a guidance that restricts the sale of unauthorized pod or cartridge-based e-cigarettes with a flavor other than tobacco or menthol, in order to limit youth access to nicotine-containing products ${ }^{13}$. After that, novel products have entered the market such as flavored e-cigarettes that are not pod or cartridge-based (e.g., the disposable "PUFF bar"), and nicotine-free pod attachments filled with flavor additives (e.g., "PUFF Krush") that are compatible to existing pod-based devices such as JUUL and thereby allow users to add flavors to nicotine vaping devices ${ }^{14,15}$. Even though these products are tobacco products or clearly intended to be used with a tobacco product and do not have an authorized marketing order from the FDA, they have not actively been removed from the market so far ${ }^{15}$. The continued sales of these products thus continues youth accessibility to flavored e-cigarettes in the US.

The measure proposed by the Dutch State Secretary to ban all e-cigarette flavors except tobacco, is expected to effectively reduce attractiveness of e-cigarettes for youth and nonsmokers in the Netherlands, since non-tobacco flavors are currently widely available on the market (Chapter 2), raise interest among non-smokers (Chapter 6), and are liked more than tobacco flavors by youth non-smokers (Chapter 8). On the other hand, this ban on all flavors except tobacco will also reduce attractiveness of e-cigarettes for current smokers: while smokers most often initiated e-cigarette use with tobacco flavors, they seem to switch from tobacco flavors towards using fruit and other sweet flavors later in time (Chapter 6) ${ }^{16-19}$. Furthermore, smokers disliked the smell of tobacco-flavored e-liquids, and liked sweet and minty flavors significantly more (Chapter 8).

It is important for the Dutch Ministry of Health, Welfare and Sport to clearly define "flavor" in the upcoming ban on all e-liquid flavors other than tobacco, and to decide how the ban will be enforced. Options to are to focus on (1) the flavor name as mentioned on the product label, (2) the flavor as perceived, and/or (3) the flavoring ingredients that result in the perceived flavor. The first option would be part of a plain packaging rule, which is considered to be implemented in 2022 as part of the National Prevention Agreement ${ }^{10}$. However, banning flavor names on package labels does not mean that the product has no flavor, which makes the second and third option more effective. The second option, to base the ban on flavor perception, requires an enforcement approach that is similar to the one established by the European Commission 
regarding the ban on characterizing flavors in combustible cigarettes and roll-your-own tobacco products ${ }^{20,21}$. Challenging considerations are associated with this approach to distinguishing characterizing from tobacco flavors: for example, which products are selected to represent a "tobacco" reference flavor and what are the perceptional and statistical boundaries of "tobacco flavor" ${ }^{20}$ ? Although this approach would be ideal since flavor is by definition a concept of perception, it is extremely time-consuming and expensive as it requires the training and maintenance of a sensory expert panel. The third option, regulating flavoring ingredients, could prevent the production of non-tobacco flavors for e-cigarettes, and is relatively time-efficient to enforce. Enforcement could be done by means of analyzing product information submitted by manufacturers to European Member State authorities via the EU-CEG system (similar to Chapter 4), and/or using chemical analysis of e-liquid flavorings (similar to Chapter 5). We showed that our algorithm using information from the EU-CEG system correctly predicted e-liquids having a tobacco flavor according to the product description in $97 \%$ of the cases (Chapter 4 , see Appendix Table A4.4). It should be noted that non-tobacco flavors that were incorrectly classified were most often assigned to the tobacco category by the algorithm; hence, in order to optimize accuracy, it would be advised to manually check the subset of e-liquids assigned to the tobacco category. Overall, a combination of using the EU-CEG system and chemical analysis would be recommended, since it would be conflicting to solely enforce a ban on non-tobacco e-cigarette flavors using information from the manufacturers of these products and it would be extremely time-consuming to solely use chemical analysis for enforcement. Furthermore, using chemical analysis to determine all flavorings in e-liquids requires an open screening approach, which is associated with a risk of missing flavorings due to limited sensitivity of the method (i.e., flavorings may be present but in concentrations below the chemical-analytical detection limit). Defining a list of flavorings that are permitted instead of a list of flavorings that are banned will therefore be more effective to enforce and will prevents loopholes for manufacturers ${ }^{22}$. Such a list of permitted flavorings could, for example, contain flavorings that are currently used by manufacturers in tobacco-flavored e-liquids. In order to create this list, it is recommended to extract recent data from the EU-CEG system, for example, data that were declared at the moment the ban was announced. Then, to create a list of permitted flavorings, the approaches described in this thesis could be applied to the recent EU-CEG data in order to classify e-liquids in flavor categories ( $\underline{\text { Chapter } 3}$ ) and identify the flavoring ingredients in e-liquids classified as having a tobacco flavor (Chapter 4). However, some tobacco-flavored e-liquids contain flavorings that on their own, in high concentrations, can produce a flavor other than tobacco (e.g., vanillin). For those flavorings, maximum concentrations could be enacted or regulators could decide on a case-by-case basis whether those flavorings should be on the permitted ingredient list or not. An additional option is to limit the number of flavorings that is allowed to be added to an e-liquid, since Chapter 4 and Chapter 5 showed that e-liquids from the tobacco category contained a significantly lower number of flavorings per e-liquid than e-liquids from other (mostly sweet) flavor categories. Finally, when the ban on all e-liquid flavors except tobacco is in force, it is important to investigate its implications beyond the prevalence of e-cigarette use among youth and non-smokers. For example, such a ban may affect the popularity and use of other (flavored) tobacco products, such as cigarillos, heated tobacco products, and waterpipe. In addition, e-cigarette users may start creating flavor mixtures themselves as ingredients can be purchased 
separately, which may result in an illicit market that potentially involves even greater health risks 23 .

\section{Methodological considerations}

\section{Selection of user groups}

E-cigarette use behavior and preferences may differ between user groups, and e-cigarette use differently affects the relative health risks of these user groups (see $\underline{\text { Chapter } 1 \text { ) }}{ }^{24,25}$. Hence, user status such as current use (e.g., daily, weekly, or past 30-day use) and non-use (e.g., never or not currently) should be clearly defined in reserach. However, no standardized definitions are yet available across literature ${ }^{24}$. When analyzing survey data (Chapter 6), we defined four different groups that cover both current and non-use of both cigarettes and e-cigarettes: never-users, dual users, smokers, and vapers. An important consideration regarding the selection of user groups is former use of e-cigarettes (i.e., vaping history), as this may influence outcomes related to liking and appeal. That is, smokers' vaping history, either regular use or having tried it once, may have led to negative or positive associations with e-cigarettes in general and/or the e-cigarette flavors under investigation. For this reason, we did not include irregular users and specifically defined the group of non-users as never-users in Chapter 6. In addition, the groups of smokers, dual users, and vapers consisted of current (daily or weekly) users of the product. To create mutually exclusive groups across the study, former users of cigarettes and/or e-cigarettes were not included as separate groups (i.e., someone can be a current vaper as well as former smoker). This means that vaping and smoking history were not taken into account in the groups selected in Chapter 6. Similarly, former e-cigarette use was no in- or exclusion criteria for the recruitment of smokers and non-smokers in our sensory study on liking of various e-liquid flavors (Chapter 8). We standardized participants' vaping history when comparing a smelling versus vaping methodology (Chapter 7) by only including smokers and non-smokers who had no reported history of e-cigarette use. In this study, we performed within-person comparisons to address our primary aim (i.e., to compare two methodologies); hence, we were not interested in differences across participants in, for example, vaping history or liking of the flavors per se. While not having included previous use of specific e-cigarette flavors at all or as a covariable in data analysis may be a limitation of these studies, it can also be argued that flavor associations should not be standardized or corrected for when investigating hedonics of and interest in e-cigarette flavors, since they reflect the real life situation.

\section{Selection of products}

Several issues need to be taken into account in the selection of e-liquids and e-cigarettes for research. First, as there are thousands of e-liquids in hundreds of different flavor descriptions available (see Figure 9.1 and Chapter 3 ) and it is impossible from a time and budget perspective to include all of these e-liquids in one chemical-analytical, sensory, or survey study, the selection of specific e-liquid flavors should be representative for a larger group of flavors (i.e., flavor categories). Throughout this entire thesis, e-liquid flavors were selected based on the categories of the e-liquid flavor wheel (created in Chapter 2). A strength of the studies described in this 
thesis is that all categories of the flavor wheel were represented, which optimized representation of the e-liquid market and maximized variation in the type of flavors under investigation. This is particularly important when investigating the role of flavors in attractiveness of e-cigarettes, as different people may be attracted to different type of flavors. The use of the same flavor lexicon in all studies also allows to accurately compare the overall findings across studies, as was done in the previous section. In addition, as multiple e-liquids have the same flavor name, it is important to carefully consider which product or brand should be selected as representative for that flavor. Chemical flavoring compositions likely differ between e-liquids with the same flavor label from different manufacturers ${ }^{26}$, and perception may therefore differ as well. To maximize variation, e-liquids from multiple brands were selected in the chemical-analytical and sensory studies described in this thesis.

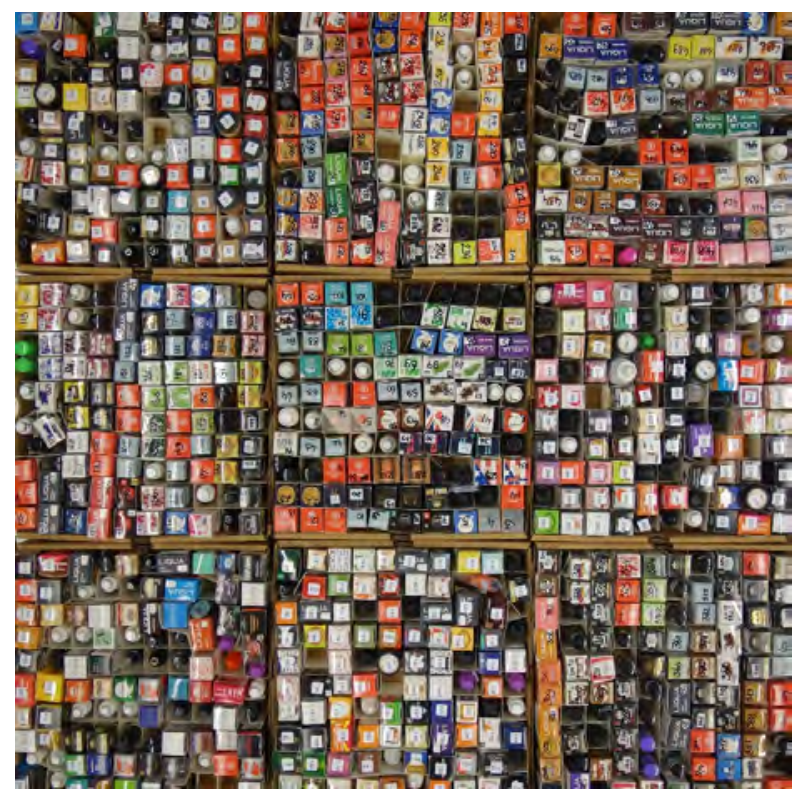

Figure 9.1: An example of the variety of available e-liquid products with different flavors and colorful packages. Image by Erna Krüsemann.

Next, when performing vaping experiments to investigate flavor perception, the e-cigarette device should be carefully selected. Some e-cigarette designs allow users to adjust settings such as the wattage, voltage and resistance. The e-cigarette device and settings influence the amount of aerosol per puff (i.e., puff volume), which determines the amounts of flavorings, nicotine, and other (potentially) toxic compounds that are delivered to the user ${ }^{27,28}$. This does not only influence exposure from a toxicological perspective, but also sensory perception and appeal ${ }^{29}$. In this thesis, non-adjustable e-cigarettes were used to standardize the amount of aerosol per 
puff, and we indicated to participants when they had to take a puff (i.e., puff frequency) (Chapter 7). To further standardize puff volume and thus perceived intensity of the e-cigarette flavors in future studies, it is recommended to also standardize puff duration, for example using a visual timer. However, it should be taken into account that this will not reflect the real life situation, and may distract participants from the actual assessment.

Finally, the e-liquids' propylene glycol to vegetable glycerin ratio (PG/VG) may influence sensory experience and nicotine delivery as well. PG is associated with smaller amounts of exhaled vapor (i.e., smaller vapor clouds) and higher nicotine exposure compared to $\mathrm{VG}^{30,31}$. Whereas some suggest that e-liquids with high PG levels may be less pleasant and satisfying than e-liquids with high VG levels ${ }^{31}$, other studies found that the PG/VG ratio had minimal impact on subjective effects and nicotine reinforcement ${ }^{32,33}$. For the vaping experiment in this thesis (Chapter 7), we selected products with a relatively high $\mathrm{PG} / \mathrm{VG}$ ratio $(70 \% \mathrm{PG}$ and $30 \%$ $\mathrm{VG}$ ) that were free of nicotine. The reason for this was that it would be unethical to expose the non-smokers in our study to nicotine and to the potentially appealing large vapor clouds. As the size of vape clouds is irrelevant in chemical-analytical and smelling experiments, we used e-liquids with varying PG/VG ratios in the studies described in Chapter 5 and Chapter 8.

\section{Reflection on methods used}

This thesis had a multidisciplinary approach to investigating the role of flavors in attractiveness of e-cigarettes. We investigated e-liquid flavor names, chemical flavoring compositions, interest in trying specific flavors as well as sensory perception of e-liquid flavors, and results were consistent overall (see previous section).

We investigated both flavoring ingredients that are added to e-liquids by manufacturers (as declared by manufacturers via the European Common Entry Gate [EU-CEG] system), and flavoring ingredients that are present in e-liquids after mixing by manufacturers (as measured by chemical analysis). Although inconsistencies between these two may exist due to potential inaccuracies reported by manufacturers and due to the formation of novel constituents after mixing ${ }^{34}$, using both methodologies has strengthened our knowledge on flavoring ingredients. We could have investigated e-cigarette aerosol as well, using a laboratory vaping machine ${ }^{35}$. The chemical composition of e-cigarette emissions may differ from the composition of the e-liquid, because novel, potentially toxic constituents may be formed during the heating process ${ }^{36}$. Therefore, analyzing e-cigarette aerosol would be more closely related to what users are exposed to, and could therefore be argued to be a more relevant approach to investigating attractiveness as well as toxicity of e-cigarettes. However, the topic of this thesis was the role of flavors in the attractiveness of e-cigarettes, and we showed that ratings for liking of the e-liquid flavor (by smelling) were strongly correlated with liking of the flavor of e-cigarette aerosol (by vaping; see Chapter 7). This justifies performing chemical analysis of flavorings in e-liquids as an approach to investigating the role of flavorings in attractiveness of e-cigarette use. In addition, analyzing flavorings in e-liquids is more closely related to the manufacturing process than analyzing compositions of the aerosol. Since current regulations on tobacco and related products focus on product manufacturing, marketing, and sales and not consumer behavior, the methodologies used in this thesis (i.e., exploring flavorings in e-liquids, using information from manufacturers and chemical analysis) are also relevant from a regulatory and enforcement perspective. 
In addition, we used information from the EU-CEG system to predict e-liquids' flavor categories with 70\% accuracy (using quantitative information) in Chapter 4. In Chapter 5, we showed that qualitative GC-MS data on e-liquid flavorings differed between categories of the e-liquid flavor wheel, which may imply that these data could be used as well to predict e-liquids' flavor categories. This means that it may be possible to classify e-liquids into flavor categories using chemical-analytical data. As a proof of principle (data not published), we applied the automatic classification approach as described in Chapter 4 to our qualitative chemical-analytical data obtained in Chapter 5. For the set of 320 e-liquids, we calculated the chance level of assigning an e-liquid to the correct category to be $9.4 \%$. Using our chemical-analytical data, the accuracy of correctly predicting an e-liquid's flavor category was $35 \%$, which exceeds chance level but is insufficient for practical application. The prediction accuracy using qualitative chemicalanalytical data was approximately half of the prediction accuracy using only the qualitative information from the EU-CEG system (66\%, see Chapter 4). A reason for this may be that only 320 e-liquids and 79 flavorings were used as input for the machine learning algorithm as compared to 16839 e-liquids and 213 flavorings in the EU-CEG study. In order to improve the accuracy of predicting e-liquids' flavor categories based on chemical-analytical data, it is recommended to greatly increase the number of e-liquids and flavorings included in the training set and/or to use quantitative instead of qualitative data. Once researchers have obtained a chemical-analytical dataset that sufficiently accurately predicts e-liquids' flavor categories, this dataset could be used to predict the flavor category of any other set of e-liquids of which the flavor description is not registered or unknown and chemical-analytical data is available. This could help countries that do not have a database such as the EU-CEG system at their disposal to obtain insight in the type of e-liquid flavors available on their market.

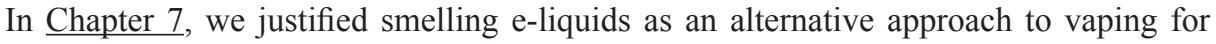
the hedonic assessment of e-liquid flavors. However, the correlation between smelling and vaping was not $100 \%$. Smelling e-cigarette aerosols (i.e., heated e-liquids) may potentially be a better approach that more closely represents vaping then smelling unheated e-liquids. However, it is practically challenging to create fresh samples of e-cigarette aerosol for all participants. Therefore, in Chapter 8 , we imitated the effect of heating by dissolving the e-liquids in demineralized water, thereby increasing the sample surface and facilitating the release of volatile odorants, and we prepared fresh samples for each participant. Another consideration regarding aerosol temperature as well as the concentration of e-liquid in water is that both are positively correlated with perceived odor intensity ${ }^{37,38}$, and perceived intensity is known to influence liking 39. A limitation of the study described in Chapter 7 is that perceived intensity of the e-liquid odors was not standardized, as the amount of drops from an e-liquid bottle may not have been a reliable indication for quantification. To address this in Chapter 8 , we standardized perceived odor intensity in a pilot experiment where participants rated perceived odor intensity of the e-liquid samples and we adjusted the amount of e-liquid drops until the mean perceived intensity was not too weak nor too strong.

Although the use of smelling as an alternative approach to vaping was justified in Chapter 7, representation of real consumer behavior was still limited due to the use of nicotine-free e-liquids. Nicotine influences sensory perception and liking of e-cigarettes through its bitter taste and irritating properties: higher nicotine concentration and thus delivery causes the user to 
experience a more intense bitter taste and harsher "throat hit", which is the term for the scratchy sensation at the back of the throat caused by nicotine ${ }^{3}$. On the other hand, an advantage of having used nicotine-free e-liquids is that it allowed us to purely focus on sensory perception and liking of the flavors, independently of the sensations caused by nicotine. In many previous publications, results about the appealing and rewarding effect of flavors in e-cigarettes are presented in relation to nicotine ${ }^{5,6,40-49}$. Whereas these studies often used nicotine-free e-liquids as well, research fully dedicated to dissociating the rewarding effects of nicotine and flavor is limited ${ }^{50}$. Therefore, our sensory (smelling) studies contribute to a better understanding of the role of flavors in attractiveness of e-cigarettes. However, in our chemical-analytical study (Chapter 6), e-liquids with various nicotine concentrations were used; nicotine was qualitatively determined but not included in the main analyses as we aimed to primarily focus on flavoring ingredients. It is yet unknown whether flavoring compositions of e-liquids differ in relation to their nicotine concentration, because, for example, more or different flavorings are added to mask the sensory effects of nicotine.

\section{Recommendations for future research}

\section{Nicotine-containing e-liquids}

Further research is needed to determine whether our results can be generalized towards nicotinecontaining e-liquids. Nicotine typically increases perceived harshness or irritation (i.e., throat hit). Smokers and vapers typically report to like the throat hit sensation, but oddly, when perception of e-cigarettes is investigated in a laboratory, throat hit or harshness or irritation is generally negatively correlated with ratings for product appeal and liking ${ }^{3}$. Therefore, liking ratings for e-liquids with nicotine might be even lower than the liking ratings we found for nicotinefree e-liquids. A potential reason for this may be that nicotine exposure during a standardized laboratory vaping session may be different from nicotine exposure that is experienced by smokers and vapers in real life.

In addition, it is unknown whether and how nicotine influences the correlation between orthonasal smelling and vaping for liking of e-liquid flavors. Further research is needed to determine whether smelling could be an alternative to vaping when investigating hedonic perception of e-liquid flavors. This could be done, for example, by determining the correlation between smelling and vaping for sensory assessment of e-liquids that contain nicotine with a design similar to the one described in Chapter 7, using experienced smokers or vapers. The correlation for liking in this study will be less strong than what we found in Chapter 7 if participants perceive the taste and sensations associated with nicotine either through smelling or vaping. If they perceive nicotine similarly through smelling and vaping, results are expected to be similar to what we found in Chapter 7. Although is expected that participants will perceive the bitter taste and harsh perception of nicotine through vaping, it is unknown whether nicotine influences perception through smelling at room temperature. St.Charles and Moldoveanu ${ }^{51}$ detected headspace concentrations of nicotine at $23{ }^{\circ} \mathrm{C}$, which may imply that participants will be exposed to nicotine when smelling e-liquids. Future research is needed to determine the influence of nicotine on sensory perception and liking by means of orthonasal smelling. This 
could be done, for example, by determining the correlations between e-liquids that are nicotinefree and (the same) e-liquids to which nicotine is added, for liking as well as harshness/irritation of the odor. As participants may be exposed to nicotine in such a study, experienced smokers or vapers should be recruited for ethical reasons.

Besides the nicotine concentration, the form in which nicotine is present in e-liquids may influence perception and liking of e-cigarettes. That is, some e-liquids, such as the one in JUUL e-cigarettes ${ }^{52}$, contain nicotine salts (i.e., protonated nicotine) instead of free-base nicotine, which more efficiently transfers nicotine to the lungs and speeds absorption of nicotine in the plasma ${ }^{53}$. Nicotine salts not only influence the addiction potential of e-cigarettes, but are also correlated with smooth sensory effects ${ }^{54}$. Future research is needed to better understand the effect of nicotine on perception and liking of e-cigarette flavors, and to determine whether this depends on the form in which nicotine is present.

\section{Tobacco-flavored e-liquids}

Further research is needed to investigate why smokers disliked tobacco flavors in e-liquids (Chapter 8) and whether this can be generalized towards all tobacco-flavored e-liquids, with and without nicotine, currently available on the market. In addition, it is unknown whether liking of tobacco-flavored e-liquids differs between smokers, dual users, and exclusive vapers. $\underline{\text { Chapter }}$ $\underline{6}$ showed that dual users, who have not (yet) quit smoking, most often initiated e-cigarette use with tobacco or menthol/mint flavors and still mostly used these flavors at a later point in time. The same applied to exclusive e-cigarette users, but they more regularly used sweet and fruity flavors at a later point in time. In line with this, literature shows that adults who started vaping tobacco flavors were less likely to quit smoking than those who vaped non-tobacco flavors ${ }^{55}$. Therefore, it can be doubted whether tobacco flavors alone will be sufficiently attractive for smokers to permanently switch towards e-cigarettes and thereby reduce their health risks. In general, according to the European Commission, evidence that e-cigarettes are an effective tool for smoking cessation is weak ${ }^{56}$. The likelihood of having quit smoking is not higher for people who use e-cigarettes as a supporting means of smoking cession than for people who use alternative tools or nothing at all ${ }^{57}$. In addition, e-cigarette users are more likely to remain dependent on nicotine ${ }^{57}$. Further research on the role of (tobacco) flavors in the effectiveness of using e-cigarettes for smoking cessation is needed. In addition, as smokers reported flavors to be the most attractive characteristic of e-cigarettes ( $\underline{\text { Chapter } 6})$, future research on the effectiveness of adding flavors to other smoking cessation tools such as nicotine gum may be interesting in order to further facilitate smoking cessation.

\section{The role of context in flavor perception and liking}

As discussed in Chapter 2 and Chapter 3 , most e-liquid flavors are sweet, and a market for savory flavors (e.g., fish, meat, cheese, and potato chips) in e-cigarettes, although popular in food ${ }^{58}$, does not seem to exist. Future research could help to better understand why people particularly like sweet e-cigarette flavors, how liking of e-cigarette flavors may differ from flavor liking in food, and whether this differs between user groups. For example, activation in reward-related brain areas could be investigated using fMRI in smokers and non-smokers, exposing them to sweet and savory odors in an e-cigarette and a food context. Odors could be presented using an 
olfactometer ${ }^{59}$, or using an e-cigarette model that is compatible with an MRI scanner such as the one developed and tested in a recent study ${ }^{60}$. Results of such a study could provide further insight in the effects of context (food vs. e-cigarette), flavor category (sweet vs. savory), as well as user group (smokers vs. non-smokers) on reward from e-cigarettes.

Finally, in the sensory studies described in $\underline{\text { Chapter } 7}$ and Chapter 8 , we aimed to minimize influences from previous experiences with the flavors under investigation in other (food) products, and therefore did not reveal the e-liquid flavor names (i.e., flavor qualities) to participants when they performed the sensory tests. This is in contrast to survey research on e-cigarette flavor preferences ( $\underline{\text { Chapter } 6}$ ), where participants typically make a mental representation of the flavor mentioned in a question or answer option based on previous experiences. On one hand, informing participants about the name of the e-liquid flavors under investigation better represents real consumer behavior, since people generally purchase e-liquids based on the flavor name presented on its package. On the other hand, as discussed in Chapter 7 and Chapter 8 , informing participants about the e-liquid flavor names under investigation may influence sensory perception and liking due to potential inconsistencies between anticipated and actual perception of the flavors. Additional research is needed to help researchers decide whether or not to disclose the e-cigarette flavors used in their study. For example, perception and liking of e-liquid flavors could be compared between settings in which participants are informed versus not informed about the flavor names, using e-liquids (actual perception) and food products with the same flavor name (anticipated perception). Results of such a study may also provide insight in the potential efficacy of implementing a plain packaging rule for e-cigarettes to reduce promotional appeal (i.e., removing all branding components, such as the product name, and attractive visual stimuli from a product's package). 


\section{Main conclusions}

This thesis aimed to investigate the role of flavors in attractiveness of e-cigarettes, with respect to different user groups. Our results showed that a large variety of e-liquid flavors is available, from menthol to watermelon, tobacco to piña colada, and from anise to cheesecake. To provide structure to the huge amount of available flavors, e-liquids can be classified into categories of our newly developed e-liquid flavor wheel. We found that the majority of e-liquids marketed in the Netherlands has a sweet flavor name, and that the flavoring ingredients most commonly added to e-liquids have a sweet aroma. Young people who do not smoke nor vape clearly prefer and like sweet and menthol/mint e-cigarette flavors, more than tobacco and other non-sweet flavors. Adults smokers reported to be mostly interested in trying e-cigarettes with a tobacco flavor, but were found to like tobacco flavors much less than sweet and menthol/mint flavors. Future research is needed to understand why smokers disliked e-liquids marketed as having a tobacco flavor, and whether this accounts for all tobacco-flavored e-liquids available. In addition, further research is needed to determine generalizability of our results towards nicotine-containing e-liquids, and to investigate the role of context (e.g., food versus e-cigarettes) in flavor perception and liking. When selecting participants, products, and methods for research on e-cigarettes and/or e-liquids, it is important to carefully consider e-liquid flavors, nicotine concentrations and PG/VG ratios, the type of e-cigarette device and settings, and participants' vaping topography and vaping history. Overall, this thesis showed that particularly non-tobacco flavors play a very important role in attractiveness of e-cigarettes, for all user groups. Therefore, banning all e-cigarette flavors except tobacco is expected to reduce attractiveness of e-cigarettes for all groups of potential e-cigarette users, including adolescent and young adult non-smokers as well as adult smokers. 


\section{References}

1. Hoffman AC, Salgado RV, Dresler C, Faller RW, Bartlett C. Flavour preferences in youth versus adults: a review. Tob Control. 2016;25(Suppl 2):ii32-ii39.

2. Steiner JE. Human Facial Expressions in Response to Taste and Smell Stimulation. In: Reese HW, Lipsitt LP, eds. Adv Child Dev Behav. Vol 13: JAI; 1979:257-295.

3. Patten T, De Biasi M. History repeats itself: Role of characterizing flavors on nicotine use and abuse. Neuropharmacology. 2020;177:108162.

4. Renner B, Schreiber K. Olfactory and trigeminal interaction of menthol and nicotine in humans. Exp Brain Res. 2012;219(1):13-26.

5. Rosbrook K, Green B. The effect of menthol on flavor intensity and nicotine irritation in an E-cigarette. Chem Senses. 2015;40(7):574.

6. Rosbrook K, Green BG. Sensory Effects of Menthol and Nicotine in an E-Cigarette. Nicotine Tob Res. 2016;18(7):1588-1595.

7. Ferris Wayne G, Connolly GN. Application, function, and effects of menthol in cigarettes: a survey of tobacco industry documents. Nicotine Tob Res. 2004;6 Suppl 1:S43-54.

8. Nationaal Expertisecentrum Tabaksontmoediging (Trimbos-instituut). Kerncijfers Roken 2019. 2020; https://www.trimbos.nl/docs/452115a7-4a93-4bd4-877a-ae4a7a4d1257.pdf.

9. Rombouts M, Van Dorsselaer S, Scheffers - van Schayck T, Tuithof M, Kleinjan M, Monshouwer K. Jeugd en riskant gedrag 2019: Kerngegevens uit het Peilstationsonderzoek Scholieren. 2020; https:// www.rijksoverheid.nl/binaries/rijksoverheid/documenten/rapporten/2020/07/01/jeugd-en-riskantgedrag-2019/VGP-206966+B.pdf.

10. Ministry of Health Welfare and Sports (the Netherlands). Nationaal Preventie Akkoord: Naar een gezonder Nederland. 2018; https:/www.rijksoverheid.nl/binaries/rijksoverheid/documenten/ convenanten/2018/11/23/nationaal-preventieakkoord/nationaal-preventieakkoord.pdf.

11. Blokhuis P. Voortgangsbrief Nationaal Preventieakkoord aan de Tweede Kamer der Staten-Generaal. 32 793, Preventief gezondheidsbeleid, nr. 484 2020; https://www.tweedekamer.nl/downloads/ document?id=a0580c79-2caa-488d-967b-f9227296d6f0\&title=Voortgangsbrief $\% 20$ Nationaal $\% 20$ Preventieakkoord.pdf.

12. World Health Organization. Factsheet - Tobacco. 2020; https://www.who.int/news-room/fact-sheets/ detail/tobacco.

13. US Food and Drug Administration. Enforcement Priorities for Electronic Nicotine Delivery Systems (ENDS) and Other Deemed Products on the Market Without Premarket Authorization (Revised). 2020; https://www.fda.gov/media/133880/download. Accessed 28 October 2020.

14. Cwalina SN, Leventhal AM, Barrington-Trimis JL. E-cigarette flavour enhancers: Flavoured pod attachments compatible with JUUL and other pod-based devices. Tob Control. 2020:tobaccocontrol-2020-055618.

15. Hemmerich N. Flavoured pod attachments score big as FDA fails to enforce premarket review. Tob Control. 2020:tobaccocontrol-2020-055718.

16. Gendall P, Hoek J. Role of flavours in vaping uptake and cessation among New Zealand smokers and non-smokers: a cross-sectional study. Tob Control. 2020:tobaccocontrol-2019-055469.

17. Gentry SV, Ward E, Dawkins L, Holland R, Notley C. Reported patterns of vaping to support longterm abstinence from smoking: a cross-sectional survey of a convenience sample of vapers. Harm Reduct J. 2020;17(1):70. 
18. Schneller LM, Bansal-Travers M, Goniewicz ML, McIntosh S, Ossip D, O'Connor RJ. Use of Flavored E-Cigarettes and the Type of E-Cigarette Devices Used among Adults and Youth in the USResults from Wave 3 of the Population Assessment of Tobacco and Health Study (2015-2016). Int J Environ Res Public Health. 2019;16(16).

19. Gravely S, Cummings KM, Hammond D, et al. The Association of E-cigarette Flavors With Satisfaction, Enjoyment, and Trying to Quit or Stay Abstinent From Smoking Among Regular Adult Vapers From Canada and the United States: Findings From the 2018 ITC Four Country Smoking and Vaping Survey. Nicotine Tob Res. 2020;22(10):1831-1841.

20. Krusemann EJZ, Lasschuijt MP, de Graaf C, et al. Sensory analysis of characterising flavours: evaluating tobacco product odours using an expert panel. Tob Control. 2019;28(2):152-160.

21. HETOC Consortium. Mapping of best practices and development of testing methods and procedures for identification of characterising flavours in tobacco products. 2016; http://ec.europa.eu/health/ tobacco/docs/hetoc_frep_en.pdf. 2016.

22. Hemmerich N, Ribisl KM, Noar SM. A List of Permissible Electronic Nicotine Delivery Systems Ingredients Would Be More Effective. Am J Public Health. 2020;110(6):774-775.

23. Erkkila BE, Kovacevic PI, Yach D. Restricting Flavors in ENDS Could Have Repercussions Beyond Youths' Use. Am J Public Health. 2020;110(6):777-778.

24. Romijnders K, van Osch L, de Vries H, Talhout R. Perceptions and Reasons Regarding E-Cigarette Use among Users and Non-Users: A Narrative Literature Review. Int J Environ Res Public Health. 2018;15(6).

25. Borland R, Murray K, Gravely S, et al. A new classification system for describing concurrent use of nicotine vaping products alongside cigarettes (so-called 'dual use'): findings from the ITC-4 Country Smoking and Vaping wave 1 Survey. Addiction. 2019;114 Suppl 1:24-34.

26. Aszyk J, Kubica P, Wozniak MK, Namiesnik J, Wasik A, Kot-Wasik A. Evaluation of flavour profiles in e-cigarette refill solutions using gas chromatography-tandem mass spectrometry. J Chromatogr A. 2018;1547:86-98.

27. DeVito EE, Krishnan-Sarin S. E-cigarettes: Impact of E-Liquid Components and Device Characteristics on Nicotine Exposure. Curr Neuropharmacol. 2018;16(4):438-459.

28. Hiler M, Karaoghlanian N, Talih S, et al. Effects of electronic cigarette heating coil resistance and liquid nicotine concentration on user nicotine delivery, heart rate, subjective effects, puff topography, and liquid consumption. Exp Clin Psychopharmacol. 2020;28(5):527-539.

29. Leventhal AM, Mason TB, Kirkpatrick MG, Anderson MK, Levine MD. E-cigarette device power moderates the effects of non-tobacco flavors and nicotine on product appeal in young adults. Addict Behav. 2020;107:106403.

30. Baassiri M, Talih S, Salman R, et al. Clouds and "throat hit": Effects of liquid composition on nicotine emissions and physical characteristics of electronic cigarette aerosols. Aerosol Sci Technol. 2017;51(11):1231-1239.

31. Spindle TR, Talih S, Hiler MM, et al. Effects of electronic cigarette liquid solvents propylene glycol and vegetable glycerin on user nicotine delivery, heart rate, subjective effects, and puff topography. Drug Alcohol Depend. 2018;188:193-199.

32. Smith TT, Heckman BW, Wahlquist AE, Cummings KM, Carpenter MJ. The Impact of E-liquid Propylene Glycol and Vegetable Glycerin Ratio on Ratings of Subjective Effects, Reinforcement Value, and Use in Current Smokers. Nicotine Tob Res. 2020;22(5):791-797. 
33. Schneller LM, Vanderbush TS, O'Connor RJ. Can Established Vapers Distinguish Different PG:VG Ratios? A Pilot Study. Tob Regul Sci. 2018;4(3):73-78.

34. Erythropel HC, Jabba SV, DeWinter TM, et al. Formation of flavorant-propylene Glycol Adducts With Novel Toxicological Properties in Chemically Unstable E-Cigarette Liquids. Nicotine Tob Res. 2019;21(9):1248-1258.

35. Famele M, Ferranti C, Abenavoli C, Palleschi L, Mancinelli R, Draisci R. The chemical components of electronic cigarette cartridges and refill fluids: review of analytical methods. Nicotine Tob Res. 2015;17(3):271-279.

36. Herrington JS, Myers C. Electronic cigarette solutions and resultant aerosol profiles. J Chromatogr A. 2015;1418:192-199.

37. Whelton AJ, Dietrich AM. Relationship between intensity, concentration, and temperature for drinking water odorants. Water Res. 2004;38(6):1604-1614.

38. Engelen L, de Wijk RA, Prinz JF, Janssen AM, Weenen H, Bosman F. The effect of oral and product temperature on the perception of flavor and texture attributes of semi-solids. Appetite. 2003;41(3):273281.

39. Moskowitz HR. Sensory intensity versus hedonic functions: classical psychophysical approaches. J Food Quality. 1981;5:109-137.

40. Audrain-McGovern J, Strasser AA, Wileyto EP. The impact of flavoring on the rewarding and reinforcing value of e-cigarettes with nicotine among young adult smokers. Drug Alcohol Depend. 2016;166:263-267.

41. Goldenson NI, Kirkpatrick MG, Barrington-Trimis JL, et al. Effects of sweet flavorings and nicotine on the appeal and sensory properties of e-cigarettes among young adult vapers: Application of a novel methodology. Drug Alcohol Depend. 2016;168:176-180.

42. Jackson A, Green B, Erythropel HC, et al. Influence of menthol and green apple e-liquids containing different nicotine concentrations among youth e-cigarette users. Exp Clin Psychopharmacol. 2020:10.1037/pha0000368.

43. Leventhal A, Cho J, Barrington-Trimis J, Pang R, Schiff S, Kirkpatrick M. Sensory attributes of e-cigarette flavours and nicotine as mediators of interproduct differences in appeal among young adults. Tob Control. 2020;29(6):679-686.

44. Leventhal AM, Goldenson NI, Barrington-Trimis JL, Pang RD, Kirkpatrick MG. Effects of nontobacco flavors and nicotine on e-cigarette product appeal among young adult never, former, and current smokers. Drug Alcohol Depend. 2019;203:99-106.

45. Mead EL, Duffy V, Oncken C, Litt MD. E-cigarette palatability in smokers as a function of flavorings, nicotine content and propylthiouracil (PROP) taster phenotype. Addict Behav. 2019;91:37-44.

46. Pullicin AJ, Kim H, Brinkman MC, Buehler SS, Clark PI, Lim J. Impacts of Nicotine and Flavoring on the Sensory Perception of E-Cigarette Aerosol. Nicotine Tob Res. 2020;22(5):806-813.

47. Palmatier MI, Smith AL, Odineal EM, Williams EA, Sheppard AB, Bradley CA. Nicotine SelfAdministration With Tobacco Flavor Additives in Male Rats. Nicotine Tob Res. 2020;22(2):224-231.

48. Cooper SY, Akers AT, Henderson BJ. Flavors enhance nicotine vapor self-administration in male mice. Nicotine Tob Res. 2020.

49. Kroemer NB, Veldhuizen MG, Delvy R, Patel BP, O’Malley SS, Small DM. Sweet taste potentiates the reinforcing effects of e-cigarettes. Eur Neuropsychopharmacol. 2018;28(10):1089-1102.

50. Rangel-Gomez M, Cruz-Cano R, Van Wagoner C, Kidanu A, McDonald CG, Clark PI. Dissociating 
the effect of flavor and nicotine in smokeless tobacco products using electroencephalography: The case of wintergreen flavors. Addict Behav. 2019;91:82-89.

51. St.Charles FK, Moldoveanu SC. The Activity and Enthalpy of Vaporization of Nicotine from Tobacco at Moderate Temperatures. Beitr Tabakforsch. 2016;27(1):11.

52. Talih S, Salman R, El-Hage R, et al. Characteristics and toxicant emissions of JUUL electronic cigarettes. Tob Control. 2019;28(6):678-680.

53. Bowen A, Xing C, Inventors; Juul Labs Inc assignee. Nicotine Salt Formulations for Aerosol Devices and Methods Thereof. US patent US 9215895B22014.

54. Gholap VV, Kosmider L, Golshahi L, Halquist MS. Nicotine forms: why and how do they matter in nicotine delivery from electronic cigarettes? Expert Opin Drug Deliv. 2020:1-10.

55. Friedman AS, Xu S. Associations of Flavored e-Cigarette Uptake With Subsequent Smoking Initiation and Cessation. JAMA Netw Open. 2020;3(6):e203826.

56. Scientific Committee on Health Environmental and Emerging Risks (SCHEER). Preliminary Opinion on electronic cigarettes. 2020; https://ec.europa.eu/health/sites/health/files/scientific_committees/ scheer/docs/scheer_o_017.pdf.

57. Chen R, Pierce JP, Leas EC, et al. E-Cigarette Use to Aid Long-Term Smoking Cessation in the US: Prospective Evidence from the PATH Cohort Study. Am J Epidemiol. 2020:kwaa161.

58. Finlayson G, Bordes I, Griffioen-Roose S, de Graaf C, Blundell JE. Susceptibility to overeating affects the impact of savory or sweet drinks on satiation, reward, and food intake in nonobese women. J Nutr. 2012;142(1):125-130.

59. Reichert JL, Postma EM, Smeets PAM, et al. Severity of olfactory deficits is reflected in functional brain networks-An fMRI study. Hum Brain Mapp. 2018;39(8):3166-3177.

60. Hobkirk AL, Bitzer Z, Goel R, et al. An Electronic Aerosol Delivery System for Functional Magnetic Resonance Imaging. Subst Abuse. 2020;14:1178221820904140. 



\section{SUMMARY}

English

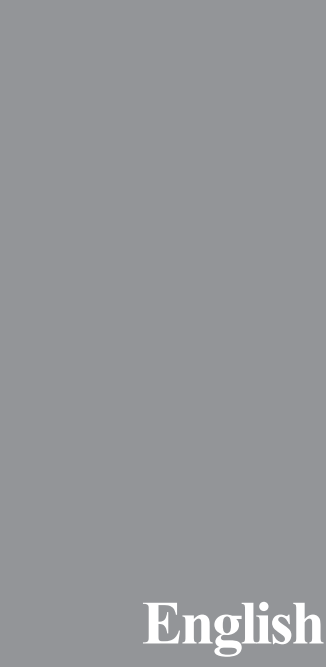


Electronic cigarettes (e-cigarettes) are devices that vaporize a liquid (e-liquid), resulting in an aerosol (vapor) that is inhaled by the product's user. E-liquids consist of a base of propylene glycol and vegetable glycerin, and mostly contain the addictive substance nicotine. E-liquids are available in many different flavors, such as pineapple, chocolate, muffin, mojito, tobacco, and hazelnut. E-cigarette use (i.e., vaping) is less harmful than smoking combustible tobacco, and therefore an attractive alternative for people who aim to quit tobacco smoking. However, e-cigarette emissions contain toxic and addictive compounds, which makes e-cigarette use not safe. The use of e-cigarettes by people who do not smoke therefore increases their health risks. Although most adult e-cigarette users in the Netherlands are concurrent or former smokers, concerns are raised that e-cigarette use also becomes increasingly popular among adolescents: at the start of this research project, more than a quarter of the secondary school students had ever used an e-cigarette.

Of all product characteristics, flavor is most important in the attractiveness of e-cigarettes for both smokers and non-smokers. Flavors in e-cigarettes are currently not regulated on European level, which causes e-liquids with appealing flavors to be widely available. Regulation of e-cigarette flavors could potentially reduce attractiveness of e-cigarettes. Whereas most e-cigarette users prefer e-liquids with a fruit, sweet, or traditional tobacco or menthol flavor, flavor preferences may differ between user groups. This may offer opportunities for regulation: if e-cigarette flavors could be identified that are attractive to smokers but not to youth and nonsmokers, regulators could decide to allow only these flavors for e-cigarettes. This way, they may be able to facilitate smoking cessation, thereby decreasing the relative health risks for smokers, while preventing the use of e-cigarettes and associated health risks among young people and nonsmokers. Therefore, the aim of this thesis was to investigate the role of flavors in attractiveness of e-cigarettes with respect to different user groups.

The first sub aim of this thesis was to structure the large amount of e-liquid flavors available. In Chapter 2, we identified and summarized e-liquid flavor names and categories mentioned in literature. We developed a flavor wheel with 16 main categories for consistent categorization of e-liquids based on their marketed flavor descriptions. In order to take regulatory decisions on e-liquid flavors, it is important to gain insight into the flavors that are available. Therefore, in Chapter 3 , we created an overview of the flavors of e-liquids marketed in the Netherlands in 2017 by classifying these e-liquids, based on information declared by manufacturers, into the categories of our flavor wheel. We found that the Dutch e-liquid market comprised nearly 20000 e-liquids in 245 different, mostly sweet, flavors.

The second sub aim of this thesis was to identify the most prevalent flavoring ingredients (flavorings) in e-liquids. In Chapter 4, we presented an overview of the flavorings that were most frequently added to e-liquids, in general and per flavor category, using information declared by manufacturers in 2017. We found that manufacturers most often add vanillin (sweet, vanillalike flavor), ethyl maltol (sweet, fruity-caramellic flavor) and ethyl butyrate (ethereal, fruity flavor) to their e-liquids, and we identified 29 flavorings that were specific to a respective flavor category. Based on the similarities and differences in e-liquid flavoring compositions between flavor categories, we could predict e-liquids' flavor categories with $70 \%$ accuracy using a machine learning algorithm. As data from manufacturers are not always complete and correct, 
we identified e-liquid flavorings in Chapter 5, using chemical analysis of 320 e-liquids classified in various flavor categories. The flavorings detected most often were vanillin, ethyl butyrate, and cis-3-hexenol (fresh, grassy flavor). In addition, we found that flavoring compositions of e-liquids were similar within fresh/sweet, warm/sweet, fresh/cooling, and non-sweet flavor categories, respectively.

The third sub aim of this thesis was to determine which flavors (potential) users of e-cigarettes prefer and like the most. In $\underline{\text { Chapter } 6}$, we conducted survey research and found that, in the Netherlands, smokers were mostly interested in e-cigarettes with a tobacco or menthol/ mint flavor, whereas people who had never smoked nor used e-cigarettes were mostly interested in sweet and menthol/mint flavors. Sweet and fruit flavors were also reported to be regularly used by people who use e-cigarettes (and do not smoke). As survey research is based on participants' memory and mental representation of how they perceive a particular flavor, this is a more indirect approach to investigating flavor preferences compared to sensory research, during which participants can actually taste or smell the product. As it is unethical to expose non-smokers and adolescents to e-cigarette emissions, research in these groups requires an alternative approach. In Chapter 7, we aimed to determine whether smelling could be an alternative to vaping in the hedonic assessment of e-cigarette flavors. We found a strong correlation between smelling and vaping for the liking of e-liquid flavors, that did not differ between smokers and non-smokers. Finally, in Chapter 8, we investigated sensory liking of various tobacco and non-tobacco e-liquid flavors in adolescent non-smokers, young adult non-smokers, and adult smokers. We found that both sweet and menthol/mint e-liquid flavors were liked similarly across all groups, and that these flavors were clearly liked more than tobacco flavors.

Further research is needed to better understand why smokers disliked e-liquids with a tobacco flavor, and whether this applies to all tobacco-flavored e-liquids available. In addition, research is needed to investigate the effect of a non-tobacco flavor ban on the effectiveness of using e-cigarettes for smoking cessation. Furthermore, since we used nicotine-free e-liquids in our sensory studies because of ethical considerations, additional research is needed to investigate the generalizability of our results towards nicotine-containing e-liquids. Finally, further research is needed on the effect of context (e.g., food versus e-cigarettes) on sensory perception and liking of flavors. This would help to better understand why sensory perception and liking seem to differ between e-liquids and food products with the same flavor names, and why sweet and not savory flavors are this popular in e-cigarettes. When selecting participants, products, and methods for studies about e-cigarettes and/or e-liquids, it is important to carefully consider (1) e-liquid flavors, nicotine concentrations and propylene glycol/vegetable glycerin ratios, (2) the type of e-cigarette device and settings, and (3) participants' vaping topography and vaping history.

In general, these findings consistently show a clear preference for sweet and menthol $/ \mathrm{mint}$ e-liquid flavors among all groups of (potential) e-cigarette users. These flavors contribute to liking and appeal of e-cigarettes directly by, respectively, enhancing sweetness and producing cooling sensations, and indirectly by masking the bitter taste and harsh/irritating sensation from nicotine. Our findings suggest that banning all e-cigarette flavors except tobacco will reduce attractiveness of e-cigarettes for all (potential) user groups, including adolescent and young adult 
non-smokers as well as adult smokers. Such a ban, as recently announced in the Netherlands, could be based on (1) the flavor as mentioned on the product label, (2) the flavor as perceived by users of the product, and/or (3) the flavoring ingredients in e-liquids that result in the perceived flavor. This could be enforced by analyzing product information declared by manufacturers in combination with chemical-analytical and/or sensory data. 



\section{SAMENVATTING}

Nederlands

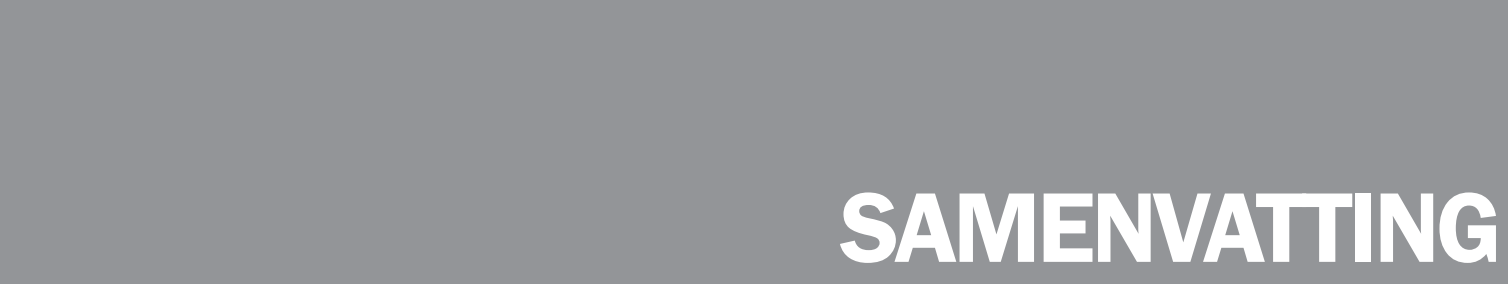


Elektronische sigaretten (e-sigaretten) zijn apparaten die een vloeistof (e-vloeistof, vanaf hier "e-liquid" genoemd) verdampen, waardoor een aerosol (damp) ontstaat die wordt ingeademd door de gebruiker van het product. E-liquids bestaan uit een basisoplossing van propyleenglycol en glycerine, en bevatten meestal de verslavende stof nicotine. E-liquids zijn verkrijgbaar in veel verschillende smaken, zoals ananas, chocolade, muffin, mojito, tabak en hazelnoot. Het gebruik van e-sigaretten (d.w.z. dampen) is minder schadelijk dan het roken van tabak en daarom een aantrekkelijk alternatief voor mensen die willen stoppen met het roken van tabak. E-sigarettendamp bevat echter toxische en verslavende stoffen, waardoor het gebruik van e-sigaretten niet veilig is. Het gebruik van e-sigaretten door mensen die niet roken verhoogt daarom hun gezondheidsrisico's. Hoewel de meeste volwassen gebruikers van e-sigaretten in Nederland tegelijkertijd roken of hebben gerookt, maakt men zich zorgen dat het gebruik van e-sigaretten ook steeds populairder wordt onder jongeren: aan het begin van dit onderzoeksproject had meer dan een kwart van de middelbare scholieren ooit een e-sigaret gebruikt.

Van alle producteigenschappen vinden zowel rokers als niet-rokers smaak en geur (vanaf nu samen aangeduid als "smaak") het belangrijkst in de aantrekkelijkheid van e-sigaretten. Smaken van e-sigaretten worden momenteel niet gereguleerd op Europees niveau, waardoor e-liquids met aantrekkelijke smaken op grote schaal verkrijgbaar zijn. Regulering van e-sigaretsmaken zou de aantrekkelijkheid van e-sigaretten kunnen verminderen. Hoewel de meeste gebruikers van e-sigaretten de voorkeur geven aan e-liquids met een fruitige, zoete, of traditionele tabakof mentholsmaak, kunnen smaakvoorkeuren verschillen tussen gebruikersgroepen. Dit biedt kansen voor regulering: als e-sigaretsmaken geïdentificeerd kunnen worden die aantrekkelijk zijn voor rokers maar niet voor jongeren en niet-rokers, dan zouden regelgevende instanties kunnen beslissen om alleen deze smaken voor e-sigaretten toe te staan. Op deze manier kunnen zij mogelijk het stoppen met roken faciliteren, en daarmee de relatieve gezondheidsrisico's voor rokers verlagen, terwijl zij het gebruik van e-sigaretten door jongeren en niet-rokers en de bijbehorende gezondheidsrisico's kunnen voorkomen. Daarom was het doel van dit proefschrift om de rol van smaken in de aantrekkelijkheid van e-sigaretten met betrekking tot verschillende gebruikersgroepen te onderzoeken.

Het eerste subdoel van dit proefschrift was het in kaart brengen van de grote hoeveelheid beschikbare e-liquidsmaken. In Hoofdstuk 2 hebben we smaaknamen en -categorieën van e-liquids die in de literatuur worden genoemd geïdentificeerd en samengevat. We hebben een zogeheten smaakwiel ontwikkeld met 16 hoofdcategorieën voor een consistente indeling van e-liquids op basis van hun geadverteerde smaakomschrijvingen. Om op het gebied van regelgeving beslissingen te kunnen nemen over e-liquidsmaken, is het belangrijk om inzicht te krijgen in de smaken die verkrijgbaar zijn. Daarom hebben we in Hoofdstuk 3 een overzicht gemaakt van de smaken van e-liquids die in 2017 in Nederland op de markt gebracht zijn. Dit hebben we gedaan door al deze e-liquids, op basis van informatie opgegeven door fabrikanten, in te delen in de categorieën van ons smaakwiel. We ontdekten dat de Nederlandse e-liquidmarkt bestaat uit bijna 20.000 e-liquids in 245 verschillende, meestal zoete, smaken.

Het tweede subdoel van dit proefschrift was het identificeren van de meest voorkomende smaakstoffen in e-liquids. In Hoofdstuk 4 presenteerden we een overzicht van de smaakstoffen die het vaakst aan e-liquids werden toegevoegd, in het algemeen en per smaakcategorie, op 
basis van informatie die door fabrikanten was opgegeven in 2017. We zagen dat fabrikanten het vaakst vanilline (zoete, vanille-achtige smaak), ethylmaltol (zoete, fruitige-karamelachtige smaak) en ethylbutyraat (etherische, fruitige smaak) aan hun e-liquids toevoegen, en we identificeerden 29 smaakstoffen die specifiek waren voor één bepaalde smaakcategorie. Op basis van de overeenkomsten en verschillen in smaakstofsamenstellingen tussen smaakcategorieën, konden we smaakcategorieën van e-liquids voorspellen met een nauwkeurigheid van $70 \%$, met behulp van een geautomatiseerd zelflerend algoritme ("machine learning"). Omdat data van fabrikanten niet altijd volledig en correct wordt opgegeven, hebben we in Hoofdstuk 5 smaakstoffen geïdentificeerd met behulp van chemische analyse van 320 e-liquids die ingedeeld waren in verschillende smaakcategorieën. De smaakstoffen die we het vaakst aantroffen waren vanilline, ethylbutyraat en cis-3-hexenol (frisse, grasachtige smaak). Bovendien ontdekten we dat smaakstofsamenstellingen van e-liquids vergelijkbaar waren binnen respectievelijk fris/ zoete, warm/zoete, fris/verkoelende en niet-zoete smaakcategorieën.

Het derde subdoel van dit proefschrift was om te bepalen welke smaken de voorkeur hebben en het lekkerst worden gevonden door (potentiële) gebruikers van e-sigaretten. In Hoofdstuk 6 hebben we vragenlijstonderzoek gedaan en zagen wij dat rokers in Nederland vooral geïnteresseerd waren in e-sigaretten met een tabak- of menthol/munt-smaak, terwijl mensen die nog nooit hadden gerookt of een e-sigaret hadden gebruikt vooral geïnteresseerd waren in zoete en menthol/munt-smaken. Mensen die e-sigaretten gebruiken (en niet roken) gaven ook aan regelmatig zoete en fruitige smaken te gebruiken. Omdat vragenlijstonderzoek gebaseerd is op het geheugen van deelnemers en hoe zij de waarneming van een bepaalde smaak mentaal inbeelden, is dit een indirectere benadering van het onderzoeken van smaakvoorkeuren ten opzichte van sensorisch onderzoek. Bij sensorisch onderzoek kunnen deelnemers het product namelijk daadwerkelijk proeven of ruiken. Omdat het ethisch niet verantwoord is om nietrokers en jongeren e-sigarettendamp te laten inhaleren, is bij onderzoek in deze groepen een alternatieve aanpak nodig. Het doel van Hoofdstuk 7 was om te bepalen of ruiken een alternatief zou kunnen zijn voor dampen bij de beoordeling van de aantrekkelijkheid van e-sigaretsmaken. Wij ontdekten dat er een sterke correlatie is tussen ruiken en dampen voor hoe lekker deelnemers een e-liquidsmaak vonden en dat deze correlatie niet verschilde tussen rokers en niet-rokers. Tenslotte onderzochten we in Hoofdstuk 8 hoe lekker de smaak van verschillende e-liquids met tabaks- en niet-tabakssmaken werd gevonden door jongere niet-rokers, jongvolwassen nietrokers en volwassen rokers. We zagen dat zowel e-liquids met zoete als menthol/munt-smaken even lekker werden gevonden door alle groepen, en dat deze smaken duidelijk lekkerder werden gevonden dan tabakssmaken.

Verder onderzoek is nodig om beter te begrijpen waarom rokers e-liquids met een tabakssmaak niet lekker vonden, en of dit geldt voor alle verkrijgbare e-liquids met een tabakssmaak. Daarnaast is onderzoek nodig om het effect te onderzoeken van een verbod op niet-tabakssmaken op de effectiviteit van de e-sigaret als middel om te stoppen met roken. Omdat wij vanwege ethische overwegingen in onze sensorische studies nicotinevrije e-liquids hebben gebruikt, is ook verder onderzoek nodig naar de generaliseerbaarheid van onze resultaten ten opzichte van nicotine-houdende e-liquids. Ten slotte is er meer onderzoek nodig naar het effect van context (bijvoorbeeld voedingsproducten ten opzichte van e-sigaretten) op de sensorische waarneming 
en aantrekkelijkheid van smaken. Dit zal helpen om beter te begrijpen waarom sensorische waarneming en aantrekkelijkheid lijkt te verschillen tussen e-liquids en voedingsproducten met dezelfde smaaknaam, en waarom zoete en niet hartige smaken zo populair zijn in e-sigaretten. Bij het selecteren van deelnemers, producten en methodes voor onderzoek naar e-sigaretten en/of e-liquids is het belangrijk om zorgvuldig na te denken over (1) de smaken, nicotine concentraties en propyleenglycol/glycerine verhoudingen in e-liquids, (2) het type apparaat en de instellingen van e-sigaretten, en (3) de damptopografie en dampgeschiedenis van de deelnemers.

In het algemeen laten deze bevindingen consequent zien dat er een duidelijke voorkeur is voor e-liquids met zoete en menthol/munt-smaken, onder alle groepen van (potentiële) e-sigaretgebruikers. Deze smaken dragen direct bij aan de aantrekkelijkheid van e-sigaretten doordat zij respectievelijk de zoetheid verhogen en een verkoelend effect veroorzaken, en indirect doordat zij de bittere smaak en de scherpe/irriterende beleving van nicotine maskeren. Onze bevindingen suggereren dat een verbod op alle e-sigaretsmaken behalve tabakssmaak de aantrekkelijkheid van e-sigaretten zal verminderen voor alle (potentiële) gebruikersgroepen, zoals jongere en jongvolwassen niet-rokers en volwassen rokers. Een dergelijk verbod, zoals onlangs aangekondigd in Nederland, kan gebaseerd worden op (1) de smaak zoals die omschreven wordt op het pakje, (2) de smaak zoals die waargenomen wordt door gebruikers van het product, en/ of (3) de smaakstoffen in e-liquids die leiden tot de waargenomen smaak. Voor de handhaving hiervan kan gebruik gemaakt worden van productinformatie die door fabrikanten is opgegeven in combinatie met chemisch-analytische en/of sensorisch data. 



\title{
Dankwoord / Acknowledgements
}

\author{
About the author \\ List of publications \\ Overview of completed training activities
}




\section{Dankwoord / Acknowledgements}

Yes! It's done! Eindelijk mag ik mijn dankwoord schrijven, terugdenkend aan vier fantastische jaren die tot dit proefschrift hebben geleid. Ik heb zoveel fijne mensen om mij heen gehad die allemaal op hun eigen manier belangrijk voor mij zijn geweest. Ik vergeet er ongetwijfeld een paar te noemen, maar voor jullie allemaal geldt: zonder jullie was het nooit zo leuk geweest!

Kees, allereerst wil ik graag jou als mijn promotor bedanken voor jouw betrokkenheid bij mijn project. Al vanaf het begin vond jij dit een interessant project: een uitstapje naar e-sigaretten binnen een afdeling over voeding en gezondheid. Door onze publicatie van het flavor wheel werd jij nog enthousiaster. Je stelde zelfs voor om een keer in jouw kantoor samen te gaan dampen (voor de wetenschap natuurlijk). Met weinig informatie gaf jij mij precies de wetenschappelijke input die ik nodig had en linkte jij onze bevindingen aan theorieën over eetgedrag. Daarnaast was je altijd geïnteresseerd in hoe het mij ging en gaf jij mij het volste vertrouwen. Dank daarvoor!

Sanne en Reinskje, wat prijs ik mijzelf gelukkig met jullie als mijn co-promotoren! Jullie vulden elkaar perfect aan en gaven elkaar de ruimte waar nodig. Onze gezamenlijke overleggen gaven mij steeds weer veel nieuwe energie en inspiratie, en waren daarnaast ook hartstikke gezellig. Sanne, ik heb heel veel van jou geleerd over sensoriek, maar ook over het opzetten van onderzoek en het geven van constructieve feedback. Ik weet niet hoe jij het deed, maar ik kreeg jouw feedback altijd sneller dan het licht! Bedankt dat jij altijd meeging in mijn enthousiasme en daar vaak zelfs nog een schepje bovenop deed. Reinskje, tijdens onze werkreizen naar Kaapstad en naar de VS heb ik jou steeds beter leren kennen. Ik heb onwijs veel bewondering voor jou als tabaksexpert en als persoon. Bedankt dat jij mij zoveel kansen hebt gegeven en dat ik alles bij jou kwijt kon. Jij dacht altijd met mij mee en kwam met creatieve ideeën. Bedankt voor jouw vertrouwen in mij!

Jeroen, ik zeg altijd: "zonder Jeroen had ik denk ik een jaar langer gedaan over mijn PhD”. Dat is misschien wat overdreven, maar ik meen het wel echt: ik ben enorm dankbaar voor jouw hulp bij de statistiek. Ik heb veel van je geleerd en vond het fijn om met jou te sparren.

I would also like to thank my opponents, Dr. Esther Croes, Prof. Dr. Vincenzo Fogliano, Prof. Dr. Suchitra Krishnan-Sarin, and Dr. Martijn Martena, for reading my thesis. I am looking forward to an interesting discussion during my defense.

Furthermore, I would like to thank Suchitra Krishnan-Sarin for the opportunity to visit you and your research group at Yale. In addition, Maciej Goniewicz, thank you for inviting me at Roswell Park for an "off the record" meeting with your research group, and for being me and my sister's tour guide. I would also like to thank Jen, Kia, Caren, and Sue for your enthusiasm during your visit to the RIVM. In addition, thank you for the opportunity to visit you and your colleagues at the FDA, and, Jen, for taking me to your workout classes ;). To all of you: I really enjoyed our discussions, and my trips to the US were definitely one of the highlights of my $\mathrm{PhD}$ ! 
Graag wil ik al mijn collega's van het RIVM, met name de tabaksgroep, bedanken voor de fijne tijd. Ik noem een aantal mensen in het bijzonder: Wouter, ik vond het heel leuk om in Wageningen samen met jou het damponderzoek uit te voeren, met name de interessante gesprekken die we hadden met de deelnemers. Peter K., bedankt voor jouw kritische blik en jouw inhoudelijke adviezen. Eric, bedankt voor het maken van de logo's van mijn Smell-e en BrainAppeal studies. Dames van het secretariaat (Janet, Irene, Louisa en Helena), bedankt voor al jullie logistieke ondersteuning. Frank, bedankt voor al jouw werk aan het GC-MS onderzoek; het was een hele klus, maar wordt onwijs gewaardeerd. Dan eindig ik met degene met wie het smaak- en geurstoffenonderzoek voor mij begon: Hans, ik vond het ontzettend leuk om na mijn stage opnieuw met jou samen te werken. Tijdens mijn PhD hebben we meer dan 800 e-liquids geanalyseerd (ook al was het meeste helaas voor de "pilot"). Bedankt voor jouw bijdrage, expertise en enthousiasme!

Lieve Anne, wat ben ik blij dat ik jou heb leren kennen! Als collega, maar ook als vriendin. Ik kan voor alles bij jou terecht, of het nu gaat om advies, feedback, even brainstormen, klagen of om mijn enthousiaste verhalen te delen. Jij bent dan altijd oprecht blij voor mij en dat waardeer ik enorm. Daarnaast heb ik ook veel met je gelachen, of het nou was om een gekke e-liquidsmaak waar we nog nooit van hadden gehoord of in de grottendisco in Cuba. Ik voel me vereerd dat jij mijn paranimf wilt zijn en hoop in de toekomst nog veel collega's zoals jij tegen te mogen komen.

Thank you to my fellow PhD candidates at RIVM: Charlotte, Christina, Coen, Laura, Alessandro, Christy, Stella, and Nienke, I really enjoyed the gezellige lunches and coffee breaks; Gina, bedankt voor jouw interesse en oneindige vrolijkheid; Astrid, ik heb genoten van alle feestjes met jou en van onze fijne gesprekken; Victoria, bedankt dat ik voor elk organisatorisch ding bij jou terecht kon en voor het sparren tijdens onze gelijktijdige afronding; en Kim, mijn roomie, bedankt dat ik zowel mijn enthousiasme als mijn frustraties bij jou kwijt kon, met als (letterlijk) hoogtepunt: de Machu Picchu!

I would also like to thank my fellow $\mathrm{PhD}$ candidates at Wageningen University, particularly Club Sense: Elbrich, Mariëlle, Matjaz, Max, Paulina, and Rachelle, I have learned so much from you about sensory science. Even though I was the only non-food scientist, you created a very safe environment for me. I've really enjoyed our Club Sense meetings, in particular the pilot tastings, celebration of successes, Christmas dinners, and the digital corona workout. Elbrich, ik wil jou daarnaast bedanken voor al jouw hulp en uitleg bij de olfactometer en MRI scanner. Paulina, thank you for helping me to find my way in the odor lab, for your boundless enthusiasm, and for all the fun we had as roommates during Pangborn.

Ook al was ik gemiddeld maar 1x per week in Helix, ik was blij dat ik dan in room 1030 kon zitten: Eva, Roelien, Janneke, Mariëlle, Marlou en Elise, bedankt voor alle gezelligheid! Marlou, ik kende jou natuurlijk al voordat ik aan mijn $\mathrm{PhD}$ begon; door jou voelde ik mij hier gelijk thuis. And to all (other) PhD colleagues in Helix: thank you for the nice chats during coffee and lunch breaks, and that you were willing to participate in my pilot experiments. 
Daarnaast wil ik graag Paul bedanken voor alle hulp bij het opzetten van de fMRI studie. Ook al is het er uiteindelijk helaas niet meer van gekomen om deze studie uit te voeren, ik heb er onwijs veel van geleerd. Henriëtte, Ineke, Mark en Anne, bedankt voor jullie ondersteuning bij onder andere de METC aanvragen en werving voor mijn sensorische studies. Gea en Jasmijn, bedankt voor jullie praktische ondersteuning en dat jullie deur letterlijk en figuurlijk altijd voor mij open stond.

Een van de leukste onderdelen van mijn $\mathrm{PhD}$ vond ik het begeleiden van studenten. Kim, Franziska, Gerlinde, Linde, Amy, Naomi en Wiebe, jullie zijn stuk voor stuk toppers die enorm veel werk hebben verzet, bedankt voor jullie inzet!

Loes, al vanaf het begin van mijn PhD leek het me heel leuk om weer met jou samen te werken. Ook al moesten we nogal wat METC- en corona-obstakels overwinnen, ik ben er trots op dat het gelukt is. Ik wil ook Wim, Erika en Kitty bedanken voor jullie input en hulp bij de Smell-e 2 studie.

Als ik dan thuis kom van werk, staan de Abstederprinssessen altijd voor me klaar. Ik wil al mijn (oud-)huisgenootjes bedanken voor alle gezelligheid: van huisweekenden en feestjes tot nieuwe kleren showen en theetjes. In het bijzonder: Laura, wat ben ik blij met jou als verdiepingsgenoot! Bedankt voor jouw luisterend oor als ik weer vol zit met enthousiaste verhalen, maar ook als ik even minder vrolijk ben. Luca, ik vind het super fijn om met jou in de combatles te staan; lekker even al onze energie kwijt en ons hoofd leeg. Dat wordt natuurlijk mede mogelijk gemaakt door de train(st)ers van onze sportschool, dus die wil ik ook graag bedanken voor hun enthousiasme tijdens de combat, tone, attack, balance, of welke les dan ook!

Mijn lieve ploeggenootjes, inmiddels zijn we al 10 jaar vriendinnen. Wat begon met roeien, bier en pasta pesto, zijn nu wijntjes en fancy (kerst)diners geworden. Jullie hebben mij de afgelopen jaren enorm gesteund. Ontspanning tijdens onze ploegweekenden, etentjes en borrels, maar ook een belletje of berichtje als het even minder lekker ging. Siets, wat ben jij een fijn persoon: altijd geïnteresseerd en in voor gezelligheid. Leo, jij bent een van de sfeermakers en zorgt dat niemand met een leeg glas wijn zit. Amber, jouw gastvrijheid en nieuwsgierigheid maakt jou betrokken en een fijn luisterend oor. Manon, mijn co-organizer van de - door corona gecancelde lustrumreis (wat zou het vet zijn geworden hè!), jij bent altijd super geïnteresseerd in mijn werk en waar ik me mee bezig hou. Bedankt dat jij doorvraagt en mij nieuwe inzichten geeft. Snoes, bedankt voor jouw lieve kaartjes en interesse in mij. Ik kan onwijs met jou lachen om bijv. de Volendammers op NPO en waardeer het enorm dat jij vaak het initiatief neemt om iets leuks te gaan doen. Miriam, ik vind het heerlijk om te zien hoe jij van het leven geniet. Dank voor jouw gezelligheid, onze geweldige girls-road trip door Kroätie en onze jaarlijkse weekendjes weg om weer even goed bij te kletsen. Tessa, mijn partner in crime, wat ben ik blij met jou als vriendin. Ik geniet enorm van al onze dansjes en drankjes, glitter shine momenten en reflectie-sessies (ja, we worden volwassen). Bedankt voor alle avonturen so far, inclusief de fantastische reis door Zuid-Afrika en heerlijke champagne-vakantie in Frankrijk. Maar bovenal: bedankt dat jij er voor mij bent op de momenten dat ik het nodig heb. 
Aan mijn bestuursgenootjes: ik ben enorm dankbaar voor onze vriendschap die na het bestuursjaar alleen maar hechter is geworden. Ook als we elkaar even wat minder vaak zien, is het altijd gelijk weer als vanouds. Patrick, Robbert, Eric en Rogier, ik had me geen betere bestuursmannen kunnen wensen. Altijd in voor een geintje, maar ook als het om serieuze dingen gaat staan jullie voor mij klaar. Suze, ik bewonder jouw eindeloze vrolijkheid en vind dat jij super goed bent in alles wat je doet. Cecile, ik waardeer het enorm dat jij altijd lekker in het nu leeft. Cocktailtje hier, zenmomentje daar; ik hoop nog heel veel avonturen met jou te mogen beleven. Katrien, voordat wij samen naar Japan waren geweest wist ik niet dat ik zo erg met jou kan lachen. Onder andere dat makt jou een fijne vakantiemaat, maar bovenal een fijne vriendin!

Van mijn verjaardagsfeest naar Gdansk: Elbrich, stiekem kennen wij elkaar ook al sinds het begin van onze Tritontijd. Ik kan altijd heerlijk met jou lachen en reflecteren, en vind jou zo'n fijn en lief persoon. Laura, wat ben ik blij dat ik jou de afgelopen jaren beter heb leren kennen. Ik moet al hardop lachen als ik 1 seconde van jouw spraakbericht heb geluisterd en geniet onwijs van onze gekke duo-dates, corona-wandelingen, jouw creativiteit en onze dansavonden thuis of in de stad.

Fien, al sinds dag 1 van onze master hebben wij een hele fijne klik. Ik geniet van onze regelmatige updates en hoop nog veel koffietjes, wandelingen en wijntjes met jou te mogen doen!

Judith, Micky en Ingrid, ook al spreken we elkaar niet meer zo vaak, wij hebben zoveel samen meegemaakt waardoor jullie altijd heel belangrijk voor mij zullen zijn.

Graag wil ik ook mijn familie bedanken voor jullie interesse en de gezellige familiedagen, -feestjes en -borrels. In het bijzonder: Geert, heel veel dank voor het maken van de cover en hoofdstukpagina's, inclusief jouw kritische blik en toffe ideeën.

Mart, ik vind het zo mooi om te zien dat onze band de laatste jaren steeds hechter is geworden. Ik vind het super gezellig dat je ook in Utrecht woont. Het allerleukste vind ik toch wel om samen met jou en/of onze vrienden te dansen in de kroeg of op een festival, en lekker te geinen. Ik kan altijd met (of om) jou lachen en kan bij jou altijd mezelf zijn. Ik geniet van onze gesprekken over het leven en ben er trots op dat ik jouw grote zus mag zijn!

Janet, je hebt geen idee hoe dankbaar ik ben dat jij vorig jaar last minute met me meeging naar de VS. Ook toen ik net mijn thesis ingeleverd had vond ik het super fijn om met jou een weekje in het NL's koninkrijk te ontspannen. Daarnaast geniet ik enorm van onze onderonsjes en kan ik nog veel leren van jouw nuchterheid. Ik vind het heel bijzonder om jou straks tijdens mijn verdediging naast me te hebben staan, bedankt dat jij dit speciale moment als mijn paranimf met mij wil delen!

Pap en mam, al vanaf het eerste moment dat ik het over deze PhD had zijn jullie mijn grootste supporters. Ik ben jullie dankbaar voor de manier waarop ik in het leven sta en de persoon die ik ben geworden. Ook wil ik jullie bedanken voor al jullie (gevraagde en ongevraagde) adviezen 
en dat jullie mij altijd gestimuleerd hebben om te doen wat ik leuk vind. Ik ben blij dat we over alles met elkaar kunnen praten en dat restaurant, kantine en hotel Krüsemann altijd voor mij geopend is! $\mathrm{xxx}$ 


\section{About the author}

Erna Johanna Zegerina Krüsemann was born on August $16^{\text {th }}, 1992$ in Sint-Oedenrode, the Netherlands. After graduating secondary school at Zwijsen College in Veghel in 2010, she started with the bachelor Biomedical Sciences at Utrecht University in Utrecht. After receiving her bachelor's degree in 2013, Erna became a full-time board member at the student rowing association U.S.R. Triton. She was responsible for all aspects regarding competitive and recreational rowing. This included chairing three committees, organizing a training camp, and organizing several other rowing-related and social activities. During 2014 - 2019, Erna remained involved in U.S.R. Triton as an advisor to

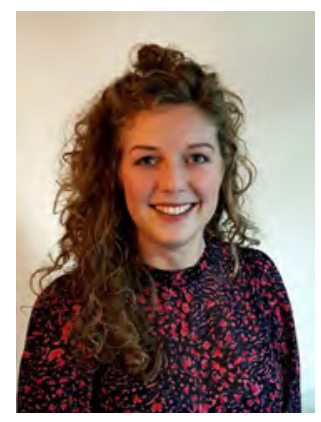
the board about the long-term strategy regarding competitive and recreational rowing.

Erna enrolled in the master Science and Business Management at Utrecht University in 2014. In the first year of this program, she did a 9-month internship at the National Institute for Public Health and the Environment (RIVM), where she developed a chemical-analytical method to identify characterizing flavors in tobacco and roll-your-own products. She also performed a sensory study on the difference threshold of menthol odor in mentholated tobacco, and was participant in the Health Effects Tobacco Composition (HETOC) consortium. In the second year of her master's program, Erna did a 6-month internship at Philips Lighting in Amsterdam / Eindhoven. Here, she wrote her graduation thesis on the implementation process of an online platform (IBM Connections) that was recently deployed within Philips Lighting in order to facilitate employee collaborations.

After receiving her master's degree in 2016, Erna worked in the Process \& Architecture EPIC team of the EPD Connect program at the St. Antonius hospital in Nieuwegein. In March 2017, Erna was appointed as a PhD candidate at the Centre for Health Protection at RIVM and the Division of Human Nutrition and Health at Wageningen University. She performed multidisciplinary research on the role of flavors in attractiveness of electronic cigarettes. During her $\mathrm{PhD}$ project, Erna supervised several bachelor and master students, and presented her research on (inter)national conferences and meetings. She volunteered at Taal Doet Meer in Utrecht as a language and future coach from 2018 to 2019. Since 2019, Erna has been a part-time board member of "Stichting Jonge Ambtenaren", where she is responsible for the program of the annual "Jonge Ambtenarendag" in the Netherlands. 


\section{List of publications}

Scientific publications:

Krüsemann EJZ, Cremers JWJM, Visser WF, Punter PH, Talhout R. The sensory difference threshold of menthol odor in flavored tobacco determined by combining sensory and chemical analysis. Chem Senses. 2017;42(3):233-238. doi: 10.1093/chemse/bjw123.

Krüsemann EJZ, Visser WF, Cremers JWJM, Pennings JLA, Talhout R. Identification of flavour additives in tobacco products to develop a flavour library. Tob Control. 2018;27:105-111. doi: 10.1136/tobaccocontrol-2016-052961.

Krüsemann EJZ, Lasschuijt MP, de Graaf C, de Wijk RA, Punter PH, van Tiel L, Cremers JWJM, van de Nobelen S, Boesveldt S, Talhout R. Sensory analysis of characterizing flavours: Evaluating tobacco product odours using an expert panel. Tob Control. 2019;28:152-160. doi: 10.1136/tobaccocontrol-2017-054152.

Krüsemann EJZ, Boesveldt S, de Graaf K, Talhout R. An e-liquid flavor wheel: A shared vocabulary based on systematically reviewing e-liquid flavor classifications in literature. Nicotine Tob Res. 2019;21(10):1310-9. doi: 10.1093/ntr/nty101.

Krüsemann EJZ, Wenng FM, Pennings JLA, de Graaf K, Talhout R, Boesveldt S. Sensory evaluation of e-liquid flavors by smelling and vaping yields similar results. Nicotine Tob Res. 2020;22(5);798-805. doi: 10.1093/ntr/ntz155.

Romijnders KAGJ and Krüsemann EJZ, Boesveldt S, de Graaf K, de Vries H, Talhout R. E-liquid flavor preferences and individual factors related to vaping: A survey among Dutch never-users, smokers, dual users, and exclusive vapers. Int J Environ Res Public Health. 2019;16(23):4661. doi: 10.3390/ijerph16234661.

Krüsemann EJZ and Havermans A, Pennings JLA, de Graaf K, Boesveldt S, Talhout R. Comprehensive overview of common e-liquid ingredients and how they can be used to predict an e-liquid's flavour category. Tob Control. E-publication ahead of print: 10 February 2020. doi: 10.1136/tobaccocontrol-2019-055447.

Krüsemann EJZ, Pennings JLA, Cremers JWJM, Bakker F, Boesveldt S, Talhout R. GC-MS analysis of e-cigarette refill solutions: A comparison of flavoring composition between flavor categories. J Pharmaceut Biomed. 2020;188:113364. doi: 10.1016/j.jpba.2020.113364.

Havermans A and Krüsemann EJZ, Pennings JLA, de Graaf K, Boesveldt S, Talhout R. Nearly 20000 e-liquids and 250 unique flavors: An overview of the Dutch market based on information from manufacturers. Tob Control. 2021;30:57-62. doi: 10.1136/tobaccocontrol-2019-055303. 
Krüsemann EJZ, van Tiel L, Pennings JLA, Vaessen W, de Graaf K, Talhout R, Boesveldt S. Both non-smoking youth and smoking adults like sweet and minty e-liquid flavors more than tobacco flavor. Submitted, 2020.

Visser WF, Krüsemann EJZ, Klerx W, Boer K, Weibolt N, Talhout R. Improving the approach to analyzing e-cigarette emissions: Detecting human "dry puff" conditions in a laboratory as validated by a panel of experienced vapers. Submitted, 2021.

Bernat JK, Jackson KJ, Krüsemann EJZ, Boesveldt S, Rudy S, de Graaf K, Talhout R. Systematic review of sensory methods to evaluate perception of flavors in tobacco and other nicotine-containing products. In preparation.

Page M, Krüsemann EJZ, Smith D, Talhout R, Goniewicz M. Comparing concentrations of popular flavoring additives in e-cigarette liquids with different advertised flavors from the United States and the Netherlands. In preparation.

\section{Other publications:}

Information brochure "E-sigaret Aantrekkelijkheid voor Rokers en Niet-rokers", March 2018. https://www.rivm.nl/documenten/informatiebrochure-aantrekkelijkheid-e-sigaretten.

Factsheet "Smaakstoffen in Tabaks- en Aanverwante Producten", June 2018. https://www.rivm.nl/documenten/smaakstoffen-tabaksproducten-factsheet.

Factsheet "Zoete Smaken Maken E-sigaretten Aantrekkelijk".

In preparation. 


\section{Overview of completed training activities}

\begin{tabular}{|c|c|c|}
\hline \multicolumn{3}{|l|}{ Discipline specific activities } \\
\hline $\begin{array}{l}\text { NutriScience. Global Nutrition: From nutrients } \\
\text { to whole diets }\end{array}$ & VLAG, Wageningen (NL) & 2017 \\
\hline Summer School on Human Olfaction & $\begin{array}{l}\text { Smell \& Taste Clinic, University } \\
\text { of Dresden Medical School, } \\
\text { Dresden (DE) }\end{array}$ & 2017 \\
\hline $\begin{array}{l}\text { Mini-symposium: Self-help for smoking } \\
\text { cessation }\end{array}$ & Trimbos Instituut, Utrecht (NL) & 2017 \\
\hline NOSE meeting & $\begin{array}{l}\text { Netherlands Olfactory Science } \\
\text { Exchange, Utrecht (NL) }\end{array}$ & 2017 \\
\hline Annual NNvT conference & $\begin{array}{l}\text { Nederlands Netwerk voor } \\
\text { Tabaksonderzoek \& Trimbos } \\
\text { Instituut, Utrecht (NL) }\end{array}$ & 2018 \\
\hline $\begin{array}{l}\text { WCToH: Uniting the world for a tobacco free } \\
\text { generation }\end{array}$ & $\begin{array}{l}\text { World Conference on Tobacco or } \\
\text { Health, Cape Town (SA) }\end{array}$ & 2018 \\
\hline $\begin{array}{l}\text { Sensory Perception \& Food Preference: The } \\
\text { role of context }\end{array}$ & VLAG, Wageningen (NL) & 2018 \\
\hline $\begin{array}{l}\text { Smarter, Faster, Stronger: Sensory \& consumer } \\
\text { science, for true business relevance }\end{array}$ & MOA, Utrecht (NL) & 2018 \\
\hline Annual NNvT conference & $\begin{array}{l}\text { Nederlands Netwerk voor } \\
\text { Tabaksonderzoek \& Trimbos } \\
\text { Instituut, Utrecht (NL) }\end{array}$ & 2019 \\
\hline WIOS symposium & $\begin{array}{l}\text { Women In Olfactory Science, } \\
\text { Wageningen (NL) }\end{array}$ & 2019 \\
\hline Pangborn Sensory Science Symposium & Elsevier, Edinburgh (UK) & 2019 \\
\hline $\begin{array}{l}\text { Meeting of the Global Tobacco Regulators } \\
\text { Forum }\end{array}$ & WHO, Bilthoven (NL) & 2019 \\
\hline Tobacco Regulatory Science Meeting & NIH, Bethesda, MD (US) & 2019 \\
\hline $\begin{array}{l}\text { Scientific meeting at the Center for Tobacco } \\
\text { Products (Office of Science) }\end{array}$ & FDA, Silver Spring, MD (US) & 2019 \\
\hline Olfactometer training & Burghart, Ede (NL) & 2020 \\
\hline Annual NNvT conference & $\begin{array}{l}\text { Nederlands Netwerk voor } \\
\text { Tabaksonderzoek \& Trimbos } \\
\text { Instituut, Utrecht (NL) }\end{array}$ & 2020 \\
\hline $\begin{array}{l}\text { Scientific meeting at the Yale Tobacco Center } \\
\text { of Regulatory Science (TCORS) }\end{array}$ & $\begin{array}{l}\text { Yale School of Medicine, New } \\
\text { Haven, CT (US) }\end{array}$ & 2020 \\
\hline $\begin{array}{l}\text { Scientific meeting at the WNY Center for } \\
\text { Research on Flavored Tobacco Products } \\
\text { (CRoFT) }\end{array}$ & $\begin{array}{l}\text { Roswell Park Comprehensive } \\
\text { Cancer Center, Buffalo, NY (US) }\end{array}$ & 2020 \\
\hline SRNT Annual Meeting & $\begin{array}{l}\text { Society for Research on Nicotine } \\
\text { and Tobacco, New Orleans, LA (US) }\end{array}$ & 2020 \\
\hline EuroSense conference & Elsevier, online (NL) & 2020 \\
\hline
\end{tabular}




\begin{tabular}{|c|c|c|}
\hline SRNT Annual Meeting & $\begin{array}{l}\text { Society for Research on Nicotine } \\
\text { and Tobacco, online (US) }\end{array}$ & 2021 \\
\hline \multicolumn{3}{|l|}{ General courses } \\
\hline RIVM $\mathrm{PhD}$ retreat & Proneri, Bilthoven (NL) & 2017 \\
\hline VLAG PhD week & VLAG, Baarlo (NL) & 2017 \\
\hline Introductiedag & RIVM, Bilthoven (NL) & 2017 \\
\hline Brain Training & WGS, Wageningen (NL) & 2017 \\
\hline Omgaan met werkstress & RIVM, Bilthoven (NL) & 2017 \\
\hline This is not a conflict! & Proneri, Bilthoven (NL) & 2017 \\
\hline Career event & Proneri, Bilthoven (NL) & 2017 \\
\hline$R$ cursus: Begin- $R$ & RIVM, Bilthoven (NL) & 2018 \\
\hline Insights training & RIVM, Bilthoven (NL) & 2019 \\
\hline Scientific integrity & Proneri, Bilthoven (NL) & 2019 \\
\hline $\mathrm{RIVM} 2^{\text {nd }} \mathrm{PhD}$ retreat & Proneri, Bilthoven (NL) & 2019 \\
\hline Team effectiviteit & Moving Performance, online (NL) & 2020 \\
\hline Lean Yellow Belt & RIVM, Bilthoven (NL) & 2020 \\
\hline $\begin{array}{l}\text { De toekomst is nu: Het nieuwe normaal, make } \\
\text { it work }\end{array}$ & $\begin{array}{l}\text { Stichting Jonge Ambtenaren, } \\
\text { online (NL) }\end{array}$ & 2020 \\
\hline \multicolumn{3}{|l|}{ Other activities } \\
\hline Preparation of research proposal & WUR, Wageningen (NL) & 2017 \\
\hline Bi-weekly chair group and ClubSense meetings & WUR, Wageningen (NL) & $2017-2021$ \\
\hline $\begin{array}{l}\text { Bi-weekly department and tobacco group } \\
\text { meetings }\end{array}$ & RIVM, Bilthoven (NL) & 2017-2021 \\
\hline $\begin{array}{l}\text { Principles of Sensory Science } \\
\text { (MSc course) }\end{array}$ & WUR, Wageningen (NL) & 2017 \\
\hline $\begin{array}{l}\text { Nutritional Neurosciences } \\
\text { (BSc course) }\end{array}$ & WUR, Wageningen (NL) & 2019 \\
\hline Organizing the "Jonge Ambtenarendag" & $\begin{array}{l}\text { Stichting Jonge Ambtenaren, } \\
\text { Maastricht (NL) }\end{array}$ & 2019-2021 \\
\hline
\end{tabular}




\section{Colophon}

The research described in this thesis was funded by the Ministry of Health, Welfare and Sport (project number 5.7.1). Financial support from Wageningen University for printing this thesis is gratefully acknowledged.

Cover by Geert Krüsemann

Printed by GVO drukkers \& vormgevers

Copyright (C) Erna J.Z. Krüsemann, 2021.

All rights are reserved. No part of this publication may be reproduced or transmitted in any form or by any means without permission from the author. 


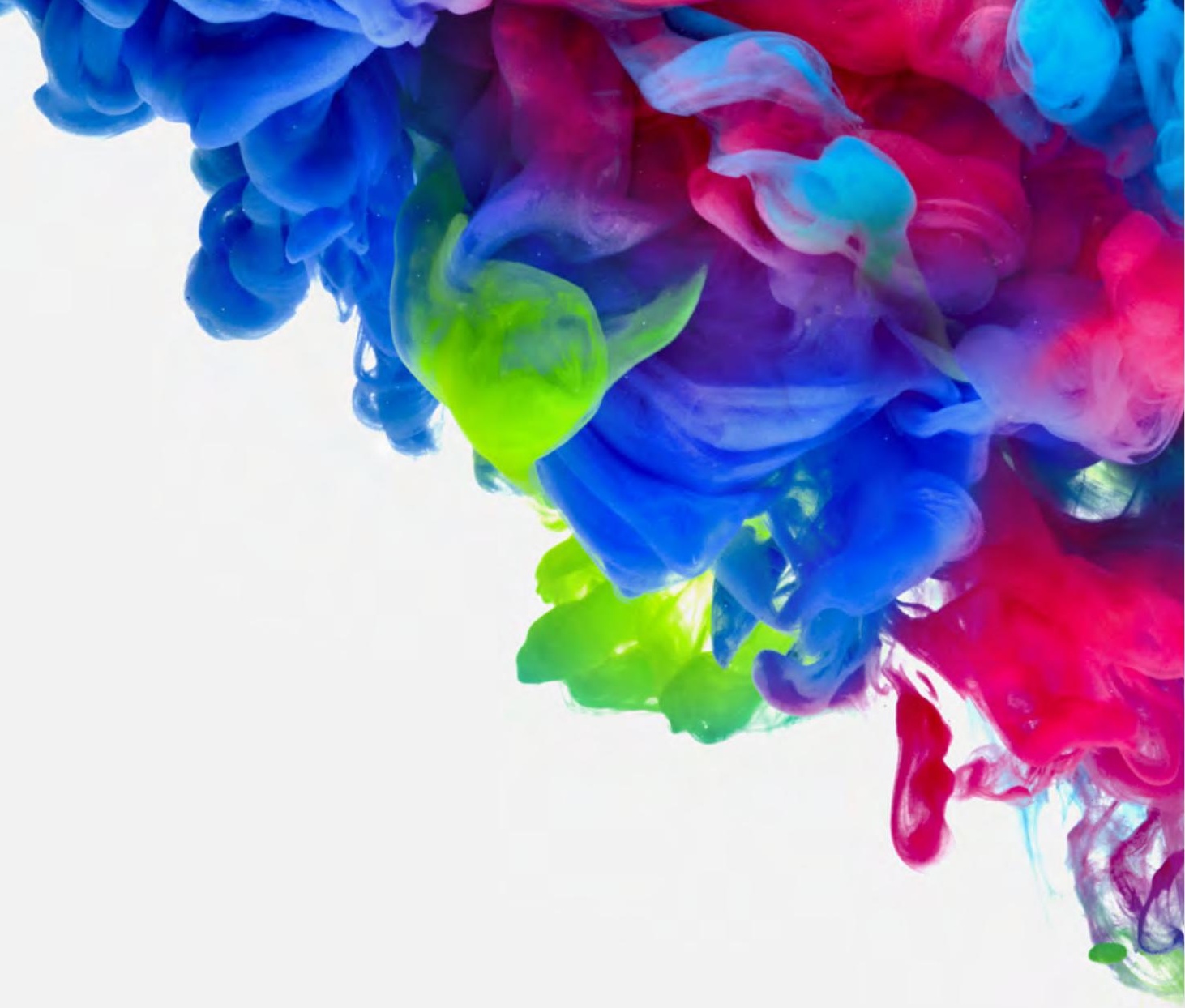

